\title{
Non-amenable finitely presented torsion-by-cyclic groups
}

\author{
A.Yu. Ol'shanskii, M.V. Sapir*
}

\begin{abstract}
We construct a finitely presented non-amenable group without free non-cyclic subgroups thus providing a finitely presented counterexample to von Neumann's problem. Our group is an extension of a group of finite exponent $n>>1$ by a cyclic group, so it satisfies the identity $[x, y]^{n}=1$.
\end{abstract}

\section{Contents}

1 Introduction $\quad 2$

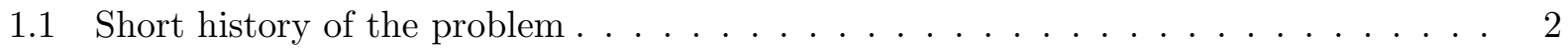

1.2 Congruence extension embeddings of the free Burnside groups . . . . . . . . . . 4

1.3 The scheme of the proof of Theorem 1.3 and the plan of the paper $\ldots \ldots . . .7$

2 Maps and diagrams $\quad 10$

2.1 Graphs and maps . . . . . . . . . . . . . . . . . . . 10

2.2 Bands . . . . . . . . . . . . . . . . . . . . . . . 11

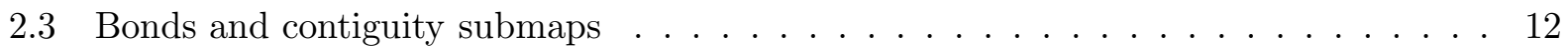

2.4 Condition A . . . . . . . . . . . . . . . . . 15

3 Burnside quotients $\quad 19$

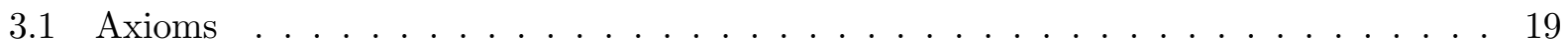

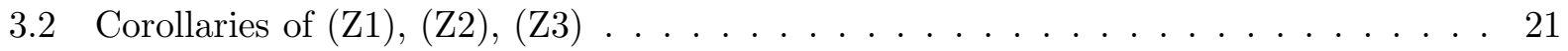

3.3 The construction of a graded presentation . . . . . . . . . . . . 27

4 Subgroups of the free Burnside groups. 42

4.1 Sets of words with small cancellation . . . . . . . . . . . . . . . . 42

4.2 Subgroups of $B(m, n)$ satisfying the congruence

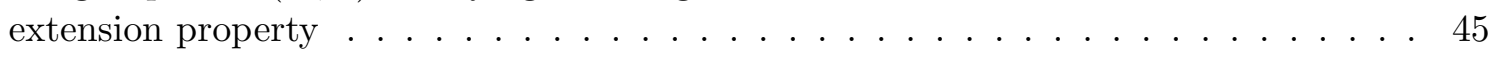

4.3 Burnside groups and free products $\ldots \ldots \ldots \ldots \ldots \ldots$

${ }^{*}$ Both authors were supported in part by the NSF grant DMS 0072307. In addition, the research of the first author was supported in part by the Russian Fund for Basic Research 99-01-00894 and by the INTAS grant, the research of the second author was supported in part by the NSF grant DMS 9978802. 
5 The presentation of the group $\quad \mathbf{5 4}$

$5.1 S$-machines and their interpretation $\ldots \ldots \ldots \ldots \ldots$

5.2 The $S$-machine $\mathbb{S} \ldots \ldots \ldots \ldots \ldots \ldots \ldots \ldots \ldots$

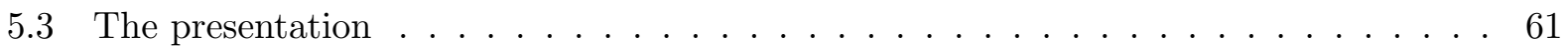

5.4 The subgroup $\langle\mathcal{A}\rangle$ of $\mathcal{H}$ is a torsion group of exponent $n \ldots \ldots \ldots$

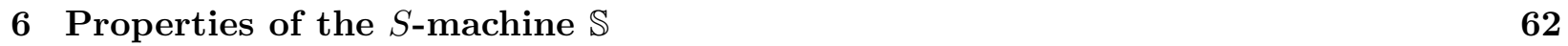

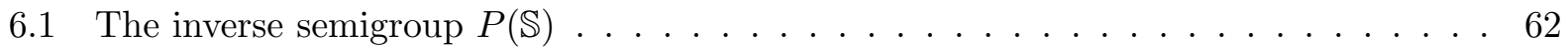

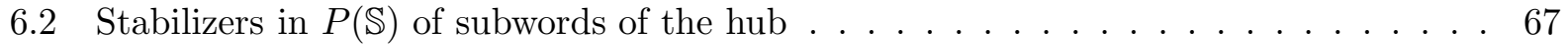

6.3 Stabilizers of arbitrary admissible words . . . . . . . . . . . . . . 70

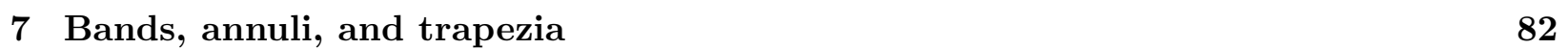

7.1 Definitions and basic facts . . . . . . . . . . . . . . . . 82

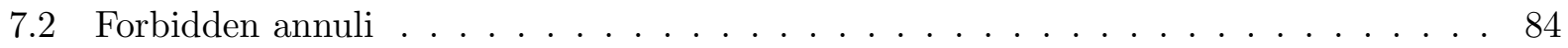

7.3 Trapezia . . . . . . . . . . . . . . . . . . . . . 85

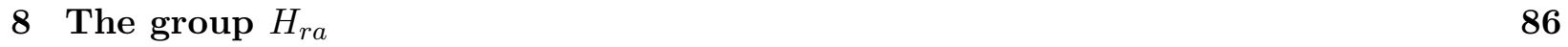

9 The group $H_{k r a} \quad 90$

9.1 Getting rid of Burnside ra-cells . . . . . . . . . . . . . . . . . . . . 90

9.2 Conditions $(\mathrm{Z} 1),(\mathrm{Z} 2),(\mathrm{Z} 3) \ldots \ldots \ldots \ldots \ldots \ldots$

$\begin{array}{ll}10 \text { Proof of Theorem 1.3 } & 101\end{array}$

$\begin{array}{ll}\text { References } & 102\end{array}$

$\begin{array}{ll}\text { Subject index } & 105\end{array}$

\section{Introduction}

\subsection{Short history of the problem}

Hausdorff [16] proved in 1914 that one can subdivide the 2-sphere minus a countable set of points into 3 parts $A, B, C$, such that each of these three parts can be obtained from each of the other two parts by a rotation, and the union of two of these parts can be obtained by rotating the third part. This implied that one cannot define a finitely additive measure on the 2-sphere which is invariant under the group $S O(3)$. In 1924 Banach and Tarski [4] generalized Hausdorff's result by proving, in particular, that in $\mathbb{R}^{3}$, every two bounded sets $A, B$ with non-empty interiors can be decomposed $A=\bigcup_{i=1}^{n} A_{i}, B=\bigcup_{i=1}^{n} B_{i}$ such that $A_{i}$ can be rotated to $B_{i}, i=1, \ldots, n$ (the so called BanachTarski paradox). Von Neumann [25] was first who noticed that the cause of the Banach-Tarski paradox is not the geometry of $\mathbb{R}^{3}$ but an algebraic property of the group $S O(3)$. He introduced the concept of an amenable group (he called such groups "measurable") as a group $G$ which has a left invariant finitely additive measure ${ }^{1} \mu, \mu(G)=1$, noticed that if a group is amenable then

\footnotetext{
${ }^{1}$ Later amenable groups were characterized in many different ways. There exist a geometric characterization by Følner [11], a characterization by Kesten [19] in terms of random walks, and a combinatorial characterization by Grigorchuk [14] among others.
} 
any set it acts upon freely also has an invariant measure and proved that a group is not amenable provided it contains a free non-abelian subgroup. He also showed that groups like $P S L(2, \mathbb{Z})$, $S L(2, \mathbb{Z})$ contain free non-abelian subgroups. So analogs of Banach-Tarski paradox can be found in $\mathbb{R}^{2}$ and even $\mathbb{R}$. Von Neumann showed that the class of amenable groups contains abelian groups, finite groups and is closed under taking subgroups, extensions, and infinite unions of increasing sequences of groups. Day and Specht showed that this class is closed under homomorphic images. The class of groups without free non-abelian subgroups is also closed under these operations and contains abelian and finite groups.

The problem of existence of non-amenable groups without non-abelian free subgroups probably goes back to von Neumann and became known as the "von Neumann problem" in the fifties. As far as we know, the first paper where this problem was formulated was the paper by Day [10]. It is also mentioned in the monograph by Greenleaf [13] based on his lectures given in Berkeley in 1967. Tits [38] proved that every non-amenable matrix group over a field of characteristic 0 contains a non-abelian free subgroup. In particular every semisimple Lie group over a field of characteristic 0 contains such a subgroup (see also Vershik [39]).

First counterexamples to the von Neumann problem were constructed by Ol'shanskii [29]. He proved that the groups with all proper subgroups cyclic constructed by him, both torsion-free [27] and torsion [28] (the so called "Tarski monsters"), are not amenable. Later Adian [1] showed that the free Burnside group of odd exponent $n \geq 665$ with at least two generators (that is the group $B(m, n)$ given by $m$ generators and all relations of the form $v^{n}=1$ where $v$ is a word in generators) is not amenable. It is interesting that the possibility of using torsion groups and, in particular, free Burnside groups as potential counterexamples to von Neumann's problem was mentioned by Day [10], conjectured by Kesten [20], and by B.H. Neumann's in his review of Specht's paper [37] in Zentralblatt.

Both Ol'shanskii's and Adian's examples are not finitely presented: in the modern terminology these groups are inductive limits of word hyperbolic groups, but they are not hyperbolic themselves. Since many mathematicians (especially topologists) are mostly interested in groups acting "nicely" on manifolds, it is natural to ask if there exists a finitely presented non-amenable group without non-abelian free subgroups. This question was explicitly formulated, for example, by Grigorchuk in [22] and by Cohen in [9]. This question is one of a series of similar questions about finding finitely presented "monsters", i.e. groups with unusual properties. Probably the most famous problem in that series is the problem about finding a finitely presented infinite torsion group. Other similar problems ask for finitely presented divisible group (group where every element has roots of every degree), finitely presented Tarski monster, etc. In each case a finitely generated example can be constructed as a limit of hyperbolic groups (see [30]), and there is no hope to construct finitely presented examples as such limits.

One difficulty in constructing a finitely presented non-amenable group without free non-abelian subgroups is that there are "very few" known finitely presented groups without free non-abelian subgroups. Most non-trivial examples are solvable or "almost" solvable (see [21]), and so they are amenable. The only known examples of finitely presented groups without free non-abelian subgroups for which the problem of amenability is non-trivial, are R.Thompson's group $F$ and its close "relatives". The fact that $F$ does not contain free non-abelian subgroups was proved by Brin and Squier in [6]. A conjecture that $F$ is not amenable was formulated first by Geoghegan [12]. A considerable amount of work has been done to prove this conjecture (see [7]) but it is still open.

One approach to constructing a finitely presented counterexample to the von Neumann problem 
would be in using the Higman embedding theorem which states that every recursively presented group can be embedded into a finitely presented group. So one can take a known finitely generated non-amenable group without non-abelian free subgroups and embed it into a finitely presented group. Of course, the resulting group will be non-amenable since the class of amenable groups is closed under taking subgroups. Unfortunately all known constructions of Higman embeddings (see, for example, [34]) use amalgamated products and non-ascending HNN extensions, which immediately leads to non-abelian free subgroups. Nevertheless Higman-like embeddings play an important role in our construction.

Our main result is the following.

Theorem 1.1. For every sufficiently large odd $n$, there exists a finitely presented group $\mathcal{G}$ which satisfies the following conditions.

1. $\mathcal{G}$ is an ascending $H N N$ extension of a finitely generated infinite group of exponent $n$.

2. $\mathcal{G}$ is an extension of a non-locally finite group of exponent $n$ by an infinite cyclic group.

3. $\mathcal{G}$ contains a subgroup isomorphic to a free Burnside group of exponent $n$ with 2 generators.

4. $\mathcal{G}$ is a non-amenable finitely presented group without free non-cyclic subgroups.

Notice that part 1 of Theorem 1.1 immediately implies part 2. By a theorem of Adian [1], part 3 implies that $\mathcal{G}$ is not amenable. Thus parts 2 and 3 imply part 4 .

Note that the first example of a finitely presented group which is a cyclic extension of an infinite torsion group was constructed by Grigorchuk [15]. But the torsion subgroup in Grigorchuk's group does not have a bounded exponent and his group is amenable (it was the first example of a finitely presented amenable but not elementary amenable group).

In the two subsequent subsections, we present the main ideas of our construction (we simplify the notation for the sake of clarity).

\subsection{Congruence extension embeddings of the free Burnside groups}

We first formulate two theorems on embeddings of the free Burnside group $B(m, n)$ of sufficiently large ${ }^{2}$ odd exponent $n$ with $m>1$ generators, and deduce Theorem 1.1 from theorems 1.2 and 1.3 which, in turn, will be proved in sections $2-10$.

We say that a subgroup $H$ of a group $G$ satisfies the Congruence Extension Property (CEP) if any homomorphism $H \rightarrow H_{1}$ of $H$ onto a group $H_{1}$ extends to a homomorphism $G \rightarrow G_{1}$ of $G$ onto some group $G_{1}$ containing $H_{1}$ as a subgroup. Equivalently, for any normal subgroup $L$ of $H$, there exists a normal subgroup $M$ of $G$ such that $H \cap M=L$. There is another obvious reformulation, which is more convenient when dealing with defining relations. Namely, the subgroup $H$ satisfies CEP if, for any subset $S \subseteq H$, we have $H \cap S^{G}=S^{H}$ (where the normal closure of a subset $T$ in a group $K$ is denoted as $T^{K}$ ).

\footnotetext{
${ }^{2}$ The number $n$ in the paper is chosen after one chooses several auxiliary parameters $\alpha>\beta>\gamma \ldots$, related by a system of inequalities. We first choose $\alpha$, then $\beta$, $\gamma$, etc. Although the values of parameters and $n$ can be calculated precisely (for example $n>10^{10}$ ), we are not doing it here because the consistency of the system of inequalities is obvious. Indeed, each inequality has the form $f(x, y, \ldots)>0$ where $x<y<\ldots$, and for already chosen positive values of $y, \ldots$, this inequality is obviously true for any sufficiently small positive value of the smallest parameter $x$ (this is the Lowest Parameter Principle from [30]).
} 
We will write $H \leq_{C E P} G$ if the subgroup $H$ of a group $G$ satisfies CEP. We also say that $H$ is CEP-embedded in $G$, or $H$ is a CEP-subgroup of $G$.

Clearly every retract $H$ of any group $G$ satisfies CEP. It is also clear that if $K$ is a CEP-subgroup of $H$ and $H$ is a CEP-subgroup of $G$ then $K$ is a CEP-subgroup of $G$.

But there exist less obvious examples. For instance, the free group $F_{\infty}$ of infinite rank can be CEP-embedded into a 2-generated free group $F_{2}$. Moreover, $F_{\infty}$ can be CEP-embedded into any non-elementary hyperbolic group [31]. This fact was used in [31] to prove that every non-elementary hyperbolic group is SQ-universal.

Theorem 1.2. For any sufficiently large odd $n$, there exists a natural number ${ }^{3} s=s(n)$ such that the free Burnside group $B(\infty, n)$ of infinite countable rank is CEP-embedded into the free Burnside group $B(s, n)$ of rank $s .^{4}$.

In the next theorem, $\mathcal{H}^{n}$ denotes the subgroup of a group $\mathcal{H}$ generated by all $n$-th powers $h^{n}$ of elements $h \in \mathcal{H}$.

Theorem 1.3. Let $n$ be a sufficiently large odd number. Then for every positive integer $s$, there is a finitely presented group $\mathcal{H}$ containing subgroup $B(s, n)$, such that

(1) $\mathcal{H}^{n} \cap B(s, n)=1$, so there exists a canonical embedding of $B(s, n)$ into $\mathcal{H} / \mathcal{H}^{n}$.

(2) the embedding of $B(s, n)$ into $\mathcal{H} / \mathcal{H}^{n}$ from (1) satisfies $C E P$.

To derive here Theorem 1.1 from Theorems 1.2 and 1.3, we assume that $n$ is a sufficiently large odd integer and $s$ is the number given by Theorem 1.2. Then let $\mathcal{H}$ be a group provided by Theorem 1.3. It has a finite presentation $\left\langle c_{1}, \ldots, c_{m} \mid \mathcal{R}\right\rangle$.

Since retracts are CEP-subgroups, we can chose a subgroup chain

$$
B(m, n) \leq_{C E P} B(m+2, n) \leq_{C E P} B(\infty, n) \leq_{C E P} B_{s}(s, n) \leq \mathcal{H}
$$

where the first and the second embeddings are given by the embeddings of the generating sets, the third and the fourth ones are given by theorems 1.2 and 1.3, respectively. Since CEP is a transitive property, we obtain from (1) and Theorem 1.3 the chain

$$
B(m, n) \leq_{C E P} B(m+2, n) \leq_{C E P} \mathcal{H}(n)
$$

The factor-group $\mathcal{H}(n)=\mathcal{H} / \mathcal{H}^{n}$ admits presentation

$$
\mathcal{H}(n)=\left\langle c_{1}, \ldots, c_{m} \mid \mathcal{R} \cup \mathcal{V}\right\rangle
$$

where $\mathcal{V}$ is the set of all $n$-th powers $v^{n}$ of group words $v=v\left(c_{1}, \ldots, c_{m}\right)$.

Let $b_{1}, \ldots, b_{m}$ and $b_{1}, \ldots, b_{m}, b_{m+1}, b_{m+2}$ be free generating sets of the subgroup $B(m, n)$ and $B(m+2, n)$, respectively, in (1) and in (2). It will be convenient to denote these subgroups of $\mathcal{H}$ (and of $\mathcal{H}(n))$ by $B_{b}(m, n)$ and by $B_{b}(m+2, n)$, respectively. There are words $w_{1}\left(c_{1}, \ldots, c_{m}\right), \ldots, w_{m}\left(c_{1}, \ldots, c_{m}\right)$ such that

$$
b_{1}=w_{1}\left(c_{1}, \ldots, c_{m}\right), \ldots, b_{m}=w_{m}\left(c_{1}, \ldots, c_{m}\right)
$$

\footnotetext{
${ }^{3}$ By using our proof of theorem 1.2 and some additional arguments, D.Sonkin recently showed that one can set $s=2$.

${ }^{4}$ Theorem 1.2 is of independent interest because it immediately implies, in particular, that every countable group of exponent $n$ is embedable into a finitely generated group of exponent $n$. This was first proved by Obraztsov (see $[30])$
} 
in $\mathcal{H}$. For every $r\left(c_{1}, \ldots, c_{m}\right) \in \mathcal{R}$, we define derived words

$$
\begin{gathered}
r^{\prime}\left(c_{1}, \ldots, c_{m}\right) \equiv r\left(w_{1}\left(c_{1}, \ldots, c_{m}\right), \ldots, w_{m}\left(c_{1}, \ldots, c_{m}\right)\right), \\
r^{\prime \prime}\left(c_{1}, \ldots, c_{m}\right) \equiv r^{\prime}\left(w_{1}\left(c_{1}, \ldots, c_{m}\right), \ldots, w_{m}\left(c_{1}, \ldots, c_{m}\right)\right), \ldots
\end{gathered}
$$

(the sign " $"$ means the letter-for-letter equality) and define $\mathcal{R}^{\prime}$ as the set of all $r^{\prime}, r^{\prime \prime}, \ldots, r^{(i)}, \ldots$ for every $r \in \mathcal{R}$. We consider the group obtained from (3) by the following formula

$$
\overline{\mathcal{H}}=\mathcal{H}(n) /\left(\mathcal{R}^{\prime}\right)^{\mathcal{H}(n)}=\left\langle c_{1}, \ldots, c_{m} \mid \mathcal{R} \cup \mathcal{V} \cup \mathcal{R}^{\prime}\right\rangle
$$

Since $B(m, n) \cong\left\langle c_{1}, \ldots, c_{m} \mid \mathcal{V}\right\rangle$, we have from $(5)$

$$
\overline{\mathcal{H}} \cong B_{c}(m, n) /\left(\mathcal{R} \cup \mathcal{R}^{\prime}\right)^{B_{c}(m, n)} .
$$

where $B_{c}(m, n)$ is the free Burnside group with $m$ free generators $c_{1}, \ldots, c_{m}$. Let $\mathcal{R}_{b}$ and $\mathcal{R}_{b}^{\prime}$ consist of copies of words from $\mathcal{R}$ and $\mathcal{R}^{\prime}$ written in letters $b_{1}, \ldots, b_{m}$. Then from (6), we immediately deduce

$$
\overline{\mathcal{H}} \cong B_{c}(m, n) /\left(\mathcal{R} \cup \mathcal{R}^{\prime}\right)^{B_{c}(m, n)} \cong B_{b}(m, n) /\left(\mathcal{R}_{b} \cup \mathcal{R}_{b}^{\prime}\right)^{B_{b}(m, n)} .
$$

Since the embedding of $B_{b}(m, n)$ into $\mathcal{H}(n)$ is an CEP-embedding (see $(2)$ ), the right-hand side of (7) is isomorphic to the group

$$
\bar{B}=B_{b}(m, n) /\left(B_{b}(m, n) \cap\left(\mathcal{R}_{b} \cup \mathcal{R}_{b}^{\prime}\right)^{\mathcal{H}(n)}\right) .
$$

However, by the definition (4), $r\left(b_{1}, \ldots, b_{m}\right)=r^{\prime}\left(c_{1}, \ldots, c_{m}\right), r^{\prime}\left(b_{1}, \ldots, b_{m}\right)=r^{\prime \prime}\left(c_{1}, \ldots, c_{m}\right), \ldots$ in $\mathcal{H}(n)$, and so $\left(\mathcal{R}_{b} \cup \mathcal{R}_{b}^{\prime}\right)^{\mathcal{H}(n)}=\left(\mathcal{R}^{\prime}\right)^{\mathcal{H}(n)}$. Hence $\bar{B}=B_{b}(m, n) /\left(B_{b}(m, n) \cap\left(\mathcal{R}^{\prime}\right)^{\mathcal{H}(n)}\right)$, i.e. $\bar{B}$ is the canonical image of the subgroup $B_{b}(m, n) \leq \mathcal{H}(n)$ in $\overline{\mathcal{H}}$. Thus, the left-hand side $\overline{\mathcal{H}}$ of $(7)$ is isomorphic to the image $\bar{B}$ of $B_{b}(m, n)$ in $\overline{\mathcal{H}}$ under the mapping $c_{i} \mapsto b_{i}, i=1, \ldots m$. (We preserve notations $c_{1}, \ldots, c_{m}, b_{1}, \ldots, b_{m}$ for the images of the generators in $\overline{\mathcal{H}}$.)

The isomorphism of $\overline{\mathcal{H}}$ with its subgroup $\bar{B}$ allows us to introduce the ascending HNN-extension

$$
\mathcal{G}=\left\langle\overline{\mathcal{H}}, t \mid t c_{i} t^{-1}=b_{i}, i=1, \ldots, m\right\rangle=\left\langle c_{1}, \ldots, c_{m} \mid \mathcal{R} \cup \mathcal{V} \cup \mathcal{R}^{\prime} \cup \mathcal{U}\right\rangle,
$$

where $\mathcal{U}=\left\{t c_{i} t^{-1} w_{i}^{-1}, i=1, \ldots, m\right\} .^{5}$ However, relators from $\mathcal{U}$ and formulas (4) imply that $\operatorname{tr}^{(i)}\left(c_{1}, \ldots, c_{m}\right) t^{-1}=r^{(i+1)}\left(c_{1}, \ldots, c_{m}\right)$ for $i \geq 0$ where $r^{(0)}=r, r^{(1)}=r^{\prime}, \ldots$ for $r \in \mathcal{R}$. Hence $\mathcal{G}=\left\langle c_{1}, \ldots, c_{m} \mid \mathcal{R} \cup \mathcal{V} \cup \mathcal{U}\right\rangle$.

Next we observe that for every word $v$ in letters $b_{1}, \ldots, b_{m}$, the relation $v\left(b_{1}, \ldots, b_{m}\right)^{n}=1$ follows from $\mathcal{R}$ because $B_{b}(m, n)$ is a subgroup of $\mathcal{H}$. Therefore $v\left(c_{1}, \ldots, c_{m}\right)^{n}=t^{-1} v\left(b_{1}, \ldots, b_{m}\right)^{n} t=1$ follows from $\mathcal{R} \cup \mathcal{U}$. Hence

$$
\mathcal{G}=\left\langle c_{1}, \ldots, c_{m} \mid \mathcal{R} \cup \mathcal{U}\right\rangle
$$

\footnotetext{
${ }^{5}$ E.Rips has been publicizing a simpler idea of constructing $\mathcal{G}$ for several years. He suggested to consider a finitely presented group $\mathcal{H}=\left\langle c_{1}, \ldots, c_{m} \mid \mathcal{R}\right\rangle$, containing $B(2, n)$, subject to relations from $\mathcal{R}$ and additional relations of the form $c_{i}=u_{i}, i=1, \ldots m$, where the words $u_{i}\left(c_{1}, \ldots, c_{m}\right)$ represent "long and complicated" elements in $B(2, n)$. The resulting group is clearly finitely presented and torsion since the images of $\mathcal{H}$ and $B(2, n)$ coincide in it. So if it were infinite, it would be an example of an infinite finitely presented torsion group (a solution of an outstanding group theory problem). If such an example were to exist, it would most certainly be non-amenable. Unfortunately in all known cases the resulting group is obviously finite, and Rips' idea has not been implemented yet. Our group (8) is not torsion but it is torsion-by-cyclic, and it satisfies the identity $[x, y]^{n}=1$.
} 
and so $\mathcal{G}$ is a finitely presented group.

The group $\mathcal{G}$ is an ascending HNN-extension of the group $\overline{\mathcal{H}}$, which is of exponent $n$ being a homomorphic image of the group $\mathcal{H}(n)$.

Finally, we notice that the canonical image $B$ of the subgroup $B_{b}(m+2, n) \leq \mathcal{H}(n)$ in $\overline{\mathcal{H}}$ is isomorphic to $B_{b}(m+2, n) /\left(\mathcal{R}^{\prime \mathcal{H}(n)} \cap B_{b}(m+2, n)\right)=B_{b}(m+2, n) /\left(\mathcal{R}^{\prime}\right)^{B_{b}(m+2, n)}$ because of the CEP-embedding $B_{b}(m+2, n) \leq_{C E P} \mathcal{H}$ (see (2)). The group $B_{b}(m+2, n) /\left(\mathcal{R}^{\prime}\right)^{B_{b}(m+2, n)}$ can be homomorphically mapped onto the free Burnside group $B(2, n)$ because all words from $\mathcal{R}^{\prime}$ are written in the first $m$ of the $m+2$ generators of $B_{b}(m+2, n)$. Therefore $B$ has a retract which is isomorphic to $B(2, n)$. (The retraction $B(2, n) \rightarrow B$ does exist since the group $B(2, n)$ is free in the variety of periodic group defined by the identity $x^{n}=1$.)

Thus, statements 1 and 3 of Theorem 1.1 (and so statements 2 and 4, as we noticed earlier) follow from Theorems 1.2 and 1.3.

Remark 1.4. The embedding of $B(s, n)$ into the group $\mathcal{H}$ in Theorem 1.3 and the set of defining relation $\mathcal{R}$ of $\mathcal{H}$ will be constructed explicitly. Since the function $f$ in Lemma 4.1 and the number of generators in Lemma 4.2 are obtained explicitly, the CEP-embedding for Theorem 1.2 is constructive too. Therefore one can also write down an explicit presentation (8) of the group $\mathcal{G}$ in Theorem 1.1. But an easier, clear and explicit construction of a finite set of the subwords $w_{1}, \ldots w_{m}$ of relators $\mathcal{U}$ from (8) is explained in Remark 4.3 which goes back to [35].

\subsection{The scheme of the proof of Theorem 1.3 and the plan of the paper}

To prove Theorem 1.3, we have to construct special embeddings of given free Burnside group $B(m, n)$ generated by a set $\mathcal{B}$ of cardinality $m$ into a finitely presented group $\mathcal{H}$ such that $\mathcal{H}^{n} \cap$ $B(m, n)=1$, and the canonical image of $B(m, n)$ in $\mathcal{H}(n)=\mathcal{H} / \mathcal{H}^{n}$ enjoys CEP. The embedding in $\mathcal{H}$ is obtained in a similar way as in our papers [33], [34] but we need a more complicated $S$-machine than in [33] ( $S$-machines were introduced by Sapir in [36]), and now the proof requires a detailed analysis of the construction of the group $\mathcal{H}(n)$.

This group is a factor-group of the group $\mathcal{H}$ generated by $\mathcal{C}=\left\{c_{1}, \ldots, c_{m}\right\}$ subject to the relations $\mathcal{R}$ of $\mathcal{H}$ over the normal subgroup generated by Burnside relations. In other words, $\mathcal{H}(n)$ is the Burnside factor of $\mathcal{H}$. Burnside factors of free groups and free products have been studied first by Adian and Novikov in [26], [3]. Geometric approach based on the notion of $A$-map was employed in the study of Burnside factors of these and more complicated groups in [30]. Papers [31], [18] extends this approach to Burnside factors of hyperbolic groups. The main problem we face in this paper is that $\mathcal{H}$ is "very" non-hyperbolic. In particular, the set of relations $\mathcal{R}$ (denoted below by $Z(\mathbb{S}, \Lambda)$ ) contains many commutativity relations, so $\mathcal{H}$ contains non-cyclic torsion-free abelian subgroups which cannot happen in a hyperbolic group.

Nevertheless (and it is one of the main ideas of the paper) one can make the Cayley graph of $\mathcal{H}$ look hyperbolic if one divides the generators from $\mathcal{C}$ into two sets and consider letters from one set as zero letters, and the corresponding edges of the Cayley graph as edges of length 0 . Thus the length of a path in the Cayley graph or in a van Kampen diagram over the presentation of $\mathcal{H}$ is the number of non-zero edges of the path.

More precisely, the group $\mathcal{H}$ is similar to the group $G(\mathbb{S})$ of [36], [34], [33]. As we mentioned above it corresponds to an $S$-machine $\mathbb{S}$. The set $\mathcal{C}$ consists of tape letters (the set $\mathbf{A}$ ), state letters (the set $\mathbf{K}$ ) and command letters (the set $\mathbf{R}$ ). Recall that unlike an ordinary Turing machine, an $S$-machine works with elements of a group, not elements of the free semigroup. 
It turns out that the most productive point of view is to consider an $S$-machine as an inverse semigroup of partial transformations of a set of states which are special (admissible) words of a certain group $H_{k a}^{*}$ generated by $\mathbf{K} \cup \mathbf{A}$. The generators of the semigroup are the $S$-rules. The machine $\mathbb{S}$ is the set of the $S$-rules. Every computation of the machine corresponds to a word over $\mathbb{S}$ which is called the history of computation, i.e. the string of commands used in the computation. With every computation $h$ applied to an admissible element $W$, one associates a van Kampen diagram $T(W, h)$ (called a trapezium) over the presentation of $H_{k a}^{*}$ (see Figure 1; a more precise picture of a trapezium is Figure 14 in Section 7.3).

The first and the last words of the computation are written on the bases the trapezium, copies of the history of the computation are written on the vertical sides. The horizontal strips (bands) of cells correspond to applications of individual rules in the computation.

The trapezia $T(W, h)$ play central role in our study of the Burnside factor $\mathcal{H}(n)$ of $\mathcal{H}$. As in [30], the main idea is to construct a graded presentation of $\mathcal{H}(n)$ where longer relations have higher ranks and such that every van Kampen diagram over the presentation of $\mathcal{H}(n)$ has the so called property A from [30]. In all diagrams over the graded presentation of $\mathcal{H}(n)$, cells corresponding to the relations from $\mathcal{R}$ are considered as 0 -cells or cells of rank $1 / 2$, and cells corresponding to Burnside relations from the graded presentation are considered as cells of ranks $1,2, \ldots$. So in these van Kampen diagrams "big" Burnside cells are surrounded by "invisible" 0-cells and "small" cells.

Last word $W \cdot h$

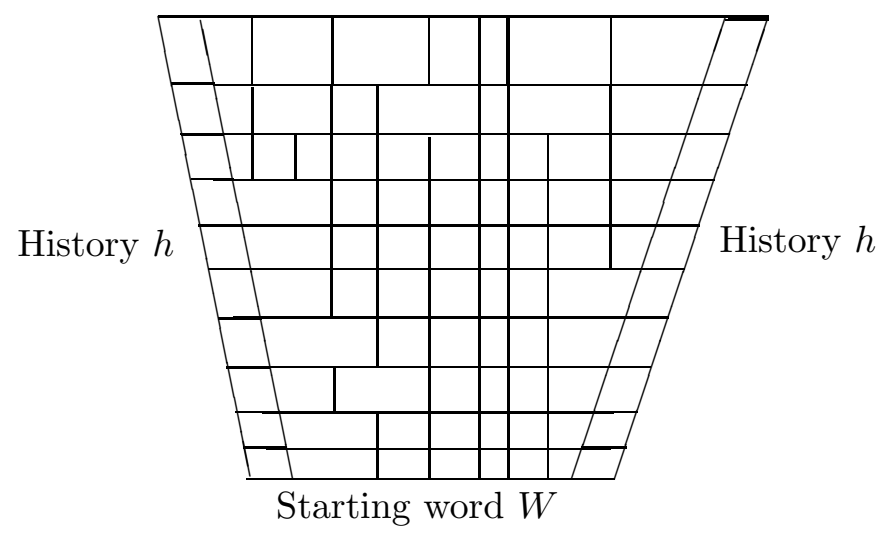

Fig. 1.

The main part of property $\mathrm{A}$ is the property that if a diagram over $\mathcal{H}(n)$ contains two Burnside cells $\Pi_{1}, \Pi_{2}$ connected by a rectangular contiguity subdiagram $\Gamma$ of rank 0 where the sides contained in the contours of the two Burnside cells are "long enough" then these two cells cancel, that is the union of $\Gamma, \Pi, \Pi^{\prime}$ can be replaced by a smaller subdiagram. This is a "graded substitute" to the classic property of small cancellation diagrams (where contiguity subdiagrams contain no cells).

Roughly speaking nontrivial contiguity subdiagrams of rank 0 turn out to be trapezia of the form $T(W, h)$ (after we clean them of Burnside 0-cells), so properties of contiguity subdiagrams can be translated into properties of the machine $\mathbb{S}$, and the inverse semigroup described above.

As an intermediate step in studying the group $\mathcal{H}(n)$, we construct a graded presentation of the Burnside factor of the subgroup of $\mathcal{H}$ generated by $\mathbf{R} \cup \mathbf{A}$. To avoid repeating the same 
arguments twice, for $\mathcal{H}$ and for the subgroup, we formulate certain key properties (Z1), (Z2), (Z3) of a presentation of a group with a separation of generators into zero and non-zero generators, so that there exists a graded presentation of the Burnside factor of the group which satisfies property $A$ (Section 3).

In order to roughly explain these conditions, consider the following example. Let $P=F_{A} \times F_{B}$ be the direct product of two free groups of rank $m$. Then the Burnside factor of $P$ is simply $B(m, n) \times B(m, n)$. Nevertheless the theory of [30] cannot be formally applied to $P$. Indeed, there are arbitrarily thick rectangles corresponding to relations $u^{-1} v^{-1} u v=1$ in the Cayley graph of $P$ so diagrams over $P$ are not A-maps in the terminology of [30] (i.e. they do not look like hyperbolic spaces). But one can obtain the Burnside factor of $P$ in two steps. First we factorize $F_{A}$ to obtain $Q=B(m, n) \times F_{B}$. After that we consider all edges labeled by letters from $A$ in the Cayley graph of $Q$ as edges of length 0 . As a result the Cayley graph of $Q$ becomes a hyperbolic space. This allows us to apply the theory of A-maps from [30] to obtain the Burnside factor of $Q$. The real reason for the theory from [30] to work in $Q$ is that $Q$ satisfies our conditions (Z1), (Z2), (Z3). But the class of groups satisfying these conditions is much bigger and includes groups corresponding to $S$-machines considered in this paper. In particular (Z3) holds in $Q$ because all 0-letters centralize $F_{B}$. This does not happen in more complicated situations. But we associate with every cyclically reduced non-0-element $w$ a "personal" subgroup $\mathbf{0}(w)$ consisting of 0-elements which is normalized by $w$.

The plan of the paper is the following. First in Section 2.2 we discuss some basic facts about van Kampen diagrams and the main tools of studying them. In Section 2, we repeat the main definitions and the main results of the theory of A-maps from [30, Chapter 5]. We also give some modifications of statements from [30] that we need later.

Then in Section 3, we introduce properties (Z1), (Z2), (Z3) and study Burnside factors. Although we follow the general scheme of [30], we encounter new significant difficulties. One of the main difficulties is that non-zero elements can be conjugates of zero elements. As a bi-product of this study, we show that under certain mild conditions, one can one can construct analogs of HNN extensions in the class of Burnside groups of sufficiently large odd exponents (see Corollary 3.40).

In Section 4, we prove some properties of the free Burnside group, in particular the existence of a countably generated free Burnside subgroup with congruence extension property (Theorem 4.4). Roughly speaking, we obtain the required embedding of the group $B(\infty, n)$ into $B(s, n)$ if we choose the images of free generators of $B(\infty, n)$ as aperiodic words $A_{1}, A_{2}, \ldots$ in generators of $B(s, n)$ satisfying a strong small cancellation condition. Here we start the inductive proof with the factor-group of the absolutely free group $F_{s}$ by arbitrary relations of form $r\left(A_{1}, A_{2}, \ldots\right)$. (This first step was described in [31].)

Then in Section 5, we define the presentation of our group $\mathcal{H}$ by listing the rules of an $S$-machine and showing how to convert the $S$-machine into a group.

In Section 6 we conduct a detailed study of the inverse semigroup corresponding to our $S$ machine. Two important properties reflect the flavor of that section and can be singled out. Lemma 6.5 (iv) says that if there is a computation starting with an element $W$, with history of the form $h^{2}$ (that is if $W$ is in the domain of $h^{2}$ ), then there exists a computation with history $h^{s}$ for every integer $s$. In other words, if a sequence of rules can be applied twice in a row, it can be applied any number of times. That lemma is used in the geometric part of the paper when we are cleaning contiguity subdiagrams of Burnside 0-cells (see Lemma 9.1). Proposition 6.17 provides an important property of stabilizers of elements in the inverse semigroup generated by the $S$-rules. It 
says, basically, that if $W$ is stabilized by $h, g h g^{-1}$ and $g^{-1} h g$ then it is stabilized by $g^{s} h g^{-s}$ for any integer $s$. This proposition is used when we consider long and narrow contiguity subdiagrams (see Lemma 9.9). Another very important feature of our $S$-machine is that two words $h_{1}$ and $h_{2}$ in the alphabet $\mathbb{S}$ define the same partial transformation on the intersection of their domains provided $h_{1}$ and $h_{2}$ are equal modulo Burnside relations (see Lemma 6.5 (ii)). Thus the work of the $S$-machine is "compatible" with Burnside relations.

In the remaining sections of the paper, we show that properties (Z1), (Z2), (Z3) are satisfied by the presentation of the group $H_{k r a}$ corresponding to our $S$-machine.

The proof ends in Section 10.

Acknowledgment. The authors are grateful to Eliyahu Rips for inspiring discussions of topics related to this paper. The authors are also grateful to Goulnara Arjantseva who read the original version of the paper and sent us numerous comments about it. This allowed us to significantly improve the exposition.

\section{Maps and diagrams}

Planar maps and van Kampen diagrams are standard tools to study complicated groups (see, for example, [23] and [30]). Here we present the main concepts related to maps and diagrams. A van Kampen diagram is a labeled map, so we start by discussing maps.

\subsection{Graphs and maps}

We are using the standard definition of a graph. In particular, every edge has a direction, and every edge has an inverse edge (having the opposite direction). For every edge $e, e_{-}$and $e_{+}$are the beginning and the end vertices of the edge.

A map is simply a finite plane graph drawn on a disc or an annulus which subdivides this surface into polygonal cells. Edges do not have labels, so no group presentations are involved in studying maps. On the other hand the theory of maps helps find the structure of van Kampen diagrams because every diagram becomes a map after we remove all the labels.

Let us recall the necessary definitions from [30].

A map is called graded if every cell $\Pi$ is assigned a non-negative number, $r(\Pi)$, its rank. A map $\Delta$ is called a map of rank $i$ if all its cells have ranks $\leq i$. Every graded map $\Delta$ has a type $\tau(\Delta)$ which is the vector whose first coordinate is the maximal rank of a cell in the map, say, $r$, the second coordinate is the number of cells of rank $r$ in the map, the third coordinate is the number of cells of rank $r-1$ and so on. We compare types lexicographically.

Let $\Delta$ be a graded map on a surface $X$. The cells of rank 0 in $\Delta$ are called 0 -cells. Some of the edges in $\Delta$ are called 0 -edges. Other edges and cells will be called positive. We define the length of a path $p$ in a map as the number of positive edges in it. The length of a path $p$ is denoted by $|p|$, in particular, $|\partial(\Pi)|$ is the perimeter of a cell $\Pi$.

We assume that

(M1) the inverse edge of a 0-edge is also a 0-edge,

(M2) either all edges in a 0-cell are 0-edges or exactly two of these edges are positive,

(M3) for every cell $\Pi$ of $\operatorname{rank} r(\Pi)>0$ the length $|\partial(\Pi)|$ of its contour is positive. 


\subsection{Bands}

A van Kampen diagram over a presentation $\langle\mathcal{X} \mid \mathcal{R}\rangle$ is a planar map with edges labeled by elements from $\mathcal{X}^{ \pm 1}$, such that the label of the contour of each cell belongs to $\mathcal{R}$ up to taking cyclic shifts and inverses [30]. In $\S 11$ of [30], 0-edges of van Kampen diagrams have label 1, but in this paper 0 -edges will be labeled also by the so called 0-letters. All diagrams which we shall consider in this paper, when considered as planar maps, will obviously satisfy conditions (M1), (M2), (M3) from Section 2.1. By van Kampen Lemma, a word $w$ over $\mathcal{X}$ is equal to 1 modulo $\mathcal{R}$ if and only if there exists a van Kampen disc diagram over $\mathcal{R}$ with boundary label $w$. Similarly (see [23]) two words $w_{1}$ and $w_{2}$ are conjugate modulo $R$ if and only if there exists an annular diagram with the internal counterclockwise contour labeled by $w_{1}$ and external counterclockwise contour labeled by $w_{2}$. There may be many diagrams with the same boundary label. Sometimes we can reduce the number of cells in a diagram or ranks of cells by replacing a 2-cell subdiagram by another subdiagram with the same boundary label. The simplest such situation is when there are two cells in a diagram which have a common edge and are mirror images of each other. In this case one can cancel these two cells (see [23]). In order strictly define this cancellation (to preserve the topological type of the diagram), one needs to use the so called 0-refinement. The 0-refinement consists of adding 0-cells with (freely trivial) boundary labels of the form $1 a a^{-1}$ or 111 (see [30], §11.5).

As in our previous papers [36], [5] and [32], one of the main tools to study van Kampen diagrams are bands and annuli.

Let $S$ be a subset of $\mathcal{X}$. An $S$-band $\mathcal{B}$ is a sequence of cells $\pi_{1}, \ldots, \pi_{n}$, for some $n \geq 0$ in a van Kampen diagram such that

- Each two consecutive cells in this sequence have a common edge labeled by a letter from $S$.

- Each cell $\pi_{i}, i=1, \ldots, n$ has exactly two $S$-edges (i.e. edges labeled by a letter from $S$ ).

- If $n=0$, then the boundary of $S$ has form $e e^{-1}$ for an $S$-edge $e$.

Figure 2 illustrates this concept. In this Figure edges $e, e_{1}, \ldots, e_{n-1}, f$ are $S$-edges, the lines $l\left(\pi_{i}, e_{i}\right), l\left(\pi_{i}, e_{i-1}\right)$ connect fixed points in the cells with fixed points of the corresponding edges.

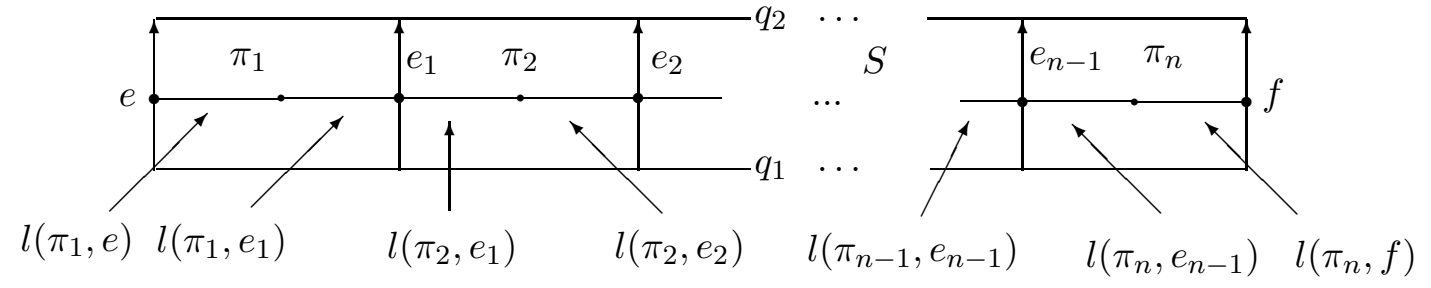

Fig. 2.

The broken line formed by the lines $l\left(\pi_{i}, e_{i}\right), l\left(\pi_{i}, e_{i-1}\right)$ connecting points inside neighboring cells is called the connecting line $e^{6}$ of the band $\mathcal{B}$. The $S$-edges $e$ and $f$ are called the start and end edges of the band.

The counterclockwise boundary of the subdiagram formed by the cells $\pi_{1}, \ldots, \pi_{n}$ of $\mathcal{B}$ has the form $e^{-1} q_{1} f q_{2}^{-1}$. We call $q_{1}$ the bottom of $\mathcal{B}$ and $q_{2}$ the top of $\mathcal{B}$, $\operatorname{denoted} \operatorname{bot}(\mathcal{B})$ and $\operatorname{top}(\mathcal{B})$.

\footnotetext{
${ }^{6}$ In [36], [5] this line was called the median of the band, but in [30], it is called the connecting line.
} 
A band $\pi_{1}, \ldots, \pi_{t}$ is called reduced if $\pi_{i+1}$ is not a mirror image of $\pi_{i}, i=1, \ldots, t-1$ (otherwise cells $\pi_{i}$ and $\pi_{i+1}$ cancel and there exists a diagram with the same boundary label as $\cup_{i} \pi_{i}$ ad containing fewer cells). Similarly, we define reduce annuli.

We say that two bands cross if their connecting lines cross. We say that a band is an annulus if its connecting line is a closed curve. In this case the first and the last cells of the band coincide (see Figure 3a).

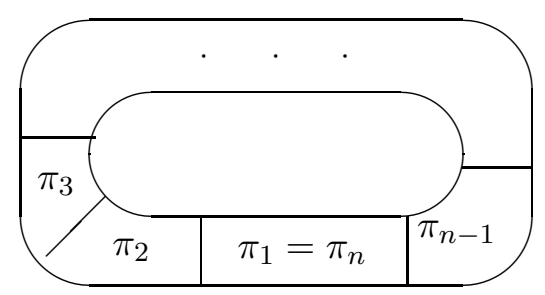

a

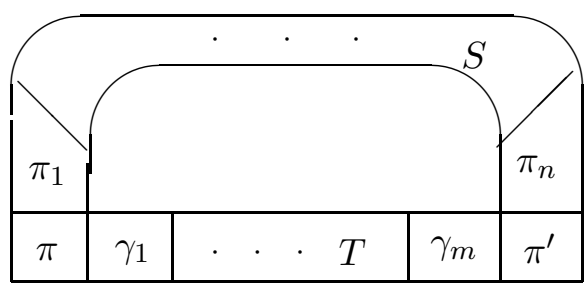

$\mathrm{b}$

Fig. 3.

If a band $\mathcal{B}$ connects two edges on the boundary of a van Kampen disc diagram $\Delta$ then the connecting line of $\mathcal{B}$ divides the region bounded by $\partial(\Delta)$ into two parts. Let $\Delta_{1}$ and $\Delta_{2}$ be the maximal subdiagrams of $\Delta$ contained in these parts. Then we say that the (connecting line of the) band $\mathcal{B}$ divides $\Delta$ into two subdiagrams $\Delta_{1}$ and $\Delta_{2}$. Notice that $\mathcal{B}$ does not belong to either $\Delta_{1}$ or $\Delta_{2}$ and $\Delta=\Delta_{1} \cup \Delta_{2} \cup \mathcal{B}$. Similarly if $\Delta$ is an annular diagram and $\mathcal{B}$ is an annulus surrounding the hole of $\Delta$ then (the connecting line of) $\mathcal{B}$ divides $\Delta$ into two annular subdiagrams.

The maximal subdiagram bounded by the connecting line of an annulus in a disc diagram is called the inside diagram of this annulus.

Let $S$ and $T$ be two disjoint subsets of $\mathcal{X}$, let $\left(\pi, \pi_{1}, \ldots, \pi_{n}, \pi^{\prime}\right)$ be an $S$-band and let $\left(\pi, \gamma_{1}\right.$, $\left.\ldots, \gamma_{m}, \pi^{\prime}\right)$ is a $T$-band. Suppose that:

- the connecting lines of these bands form a simple closed curve,

- on the boundary of $\pi$ and on the boundary of $\pi^{\prime}$ the pairs of $S$-edges separate the pairs of T-edges,

- the start and end edges of these bands are not contained in the region bounded by the connecting lines of the bands.

Then we say that these bands form an $(S, T)$-annulus and the closed curve formed by the parts of connecting lines of these bands is the connecting line of this annulus (see Figure $3 \mathrm{~b}$ ). For every $(S, T)$-annulus we define the inside subdiagram diagram of the annulus as the maximal subdiagram bounded by the connecting line of the annulus.

\subsection{Bonds and contiguity submaps}

Now let us introduce the main concepts from Chapter 5 of [30]. Consider a map $\Delta$. A path in $\Delta$ is called reduced if it does not contain subpaths of length 2 which are homotopic to paths of length 0 
by homotopies involving only 0 -cells. We shall usually suppose that the contour of $\Delta$ is subdivided into several subpaths called sections. We usually assume that sections are reduced paths.

By property M2 if a 0-cell has a positive edge, it has exactly two positive edges. Thus we can consider bands of 0 -cells having positive edges. The start and end edges of such bands are called adjacent.

Let $e$ and $f$ be adjacent edges of $\Delta$ belonging to two positive cells $\Pi_{1}$ and $\Pi_{2}$ or to sections of the contour of $\Delta$. Then there exists a band of 0-cells connecting these edges. The union of the cells of this band is called a 0-bond between the cells $\Pi_{1}$ and $\Pi_{2}$ (or between a cell and a section of the contour of $\Delta$, or between two sections of the contour). The connecting line of the band is called the connecting line of the bond. The contour of the 0 -bond has the form $p^{-1} e s^{-1} f^{-1}$ where $p$ and $s$ are paths of length 0 because every 0 -cell has at most two positive edges.

Now suppose we have chosen two pairs $\left\{e_{1}, f_{1}\right\}$ and $\left\{e_{2}, f_{2}\right\}$ of adjacent edges such that $e_{1}, e_{2}$ belong to the contour of a cell $\Pi_{1}$ and $f_{1}^{-1}, f_{2}^{-1}$ to the contour of a cell $\Pi_{2}$. Then we have two bonds $E_{1}$ and $E_{2}$. If $E_{1}=E_{2}$ (that is $e_{1}=e_{2}, f_{1}=f_{2}$ ) then let $\Gamma=E_{1}$. Now let $E_{1} \neq E_{2}$ and $z_{1} e_{1} w_{1} f_{1}^{-1}$ and $z_{2} e_{2} w_{2} f_{2}^{-1}$ be the contours of these bonds. Further let $y_{1}$ and $y_{2}$ be subpaths of the contours of $\Pi_{1}$ and $\Pi_{2}$ (of $\Pi_{1}$ and $q$, or $q_{1}$ and $q_{2}$, where $q, q_{1}$ and $q_{2}$ are some segments of the boundary cotours) where $y_{1}$ (or $\left.y_{2}\right)$ has the form $e_{1} p e_{2}$ or $e_{2} p e_{1}\left(\right.$ or $\left(f_{1} u f_{2}\right)^{-1}$ or $\left.\left(f_{2} u f_{1}\right)^{-1}\right)$. If $z_{1} y_{1} w_{2} y_{2}$ (or $z_{2} y_{1} w_{1} y_{2}$ ) is a contour of a disc submap $\Gamma$ which does not contain $\Pi_{1}$ or $\Pi_{2}$, then $\Gamma$ is called a 0-contiguity submap of $\Pi_{1}$ to $\Pi_{2}$ (or $\Pi_{1}$ to $q$ or of $q_{1}$ to $q_{2}$ ). The contour of $\Gamma$ is naturally subdivided into four parts. The paths $y_{1}$ and $y_{2}$ are called the contiguity arcs. We write $y_{1}=\Gamma \wedge \Pi_{1}, y_{2}=\Gamma \wedge \Pi_{2}$ or $y_{2}=\Gamma \wedge q$. The other two paths are called the side arcs of the contiguity submap.

The ratio $\left|y_{1}\right| /\left|\partial\left(\Pi_{1}\right)\right|$ (or $\left|y_{2}\right| /\left|\partial\left(\Pi_{2}\right)\right|$ is called the degree of contiguity of the cell $\Pi_{1}$ to the cell $\Pi_{2}$ or to $q$ (or of $\Pi_{2}$ to $\Pi_{1}$ ). We denote the degree of contiguity of $\Pi_{1}$ to $\Pi_{2}$ (or $\Pi_{1}$ to $q$ ) by $\left(\Pi_{1}, \Gamma, \Pi_{2}\right)$ (or $\left.\left(\Pi_{1}, \Gamma, q\right)\right)$. Notice that this definition is not symmetric and when $\Pi_{1}=\Pi_{2}=\Pi$, for example, then $(\Pi, \Gamma, \Pi)$ is a pair of numbers.

A connecting line of $\Gamma$ is chosen as the connecting line of one of the bonds $E_{1}$ or $E_{2}$.

We say that two contiguity submaps are disjoint if none of them has a common point with the interior of the other one, and their contiguity arcs do not have common edges.

Now we are going to define $k$-bonds and $k$-contiguity submaps for $k>0$. In these definitions we need a fixed number $\varepsilon, 0<\varepsilon<1$.

Let $k>0$ and suppose that we have defined the concepts of $j$-bond and $j$-contiguity submap for all $j<k$. Consider three cells $\pi, \Pi_{1}, \Pi_{2}$ (possibly with $\Pi_{1}=\Pi_{2}$ ) satisfying the following conditions:

1. $r(\pi)=k, r\left(\Pi_{1}\right)>k, r\left(\Pi_{2}\right)>k$,

2. there are disjoint submaps $\Gamma_{1}$ and $\Gamma_{2}$ of $j_{1}$-contiguity of $\pi$ to $\Pi_{1}$ and of $j_{2}$-contiguity of $\pi$ to $\Pi_{2}$, respectively, with $j_{1}<k, j_{2}<k$, such that $\Pi_{1}$ is not contained in $\Gamma_{2}$ and $\Pi_{2}$ is not contained in $\Gamma_{1}$,

3. $\left(\pi, \Gamma_{1}, \Pi_{1}\right) \geq \varepsilon,\left(\pi, \Gamma_{2}, \Pi_{2}\right) \geq \varepsilon$.

Then there is a minimal submap $E$ in $\Delta$ containing $\pi, \Gamma_{1}, \Gamma_{2}$. This submap is called a $k$-bond between $\Pi_{1}$ and $\Pi_{2}$ defined by the contiguity submaps $\Gamma_{1}$ and $\Gamma_{2}$ with principal cell $\pi$ (see Figure 4). 
The contiguity arc $q_{1}$ of the bond $E$ to $\Pi_{1}$ is $\Gamma_{1} \wedge \Pi_{1}$. It will be denoted by $E \wedge \Pi_{1}$. Similarly $E \wedge \Pi_{2}$ is by definition the arc $q_{2}=\Gamma_{2} \wedge \Pi_{2}$. The contour $\partial(E)$ can be written in the form $p_{1} q_{1} p_{2} q_{2}$ where $p_{1}, p_{2}$ are called the side arcs of the bond $E$.

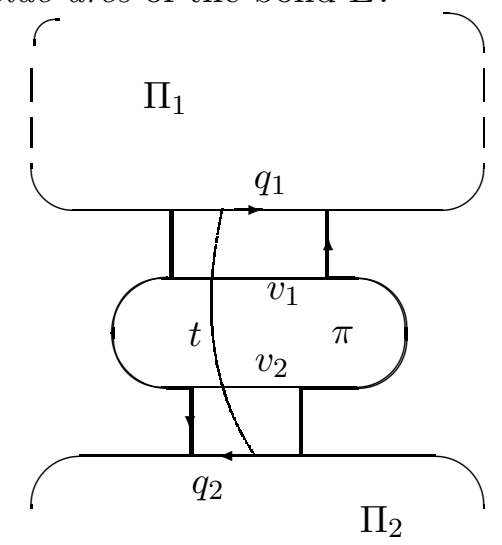

Fig. 4.

For each $k$-bond we fix a connecting line obtained by joining the terminal points of connecting lines of $\Gamma_{1}$ and $\Gamma_{2}$ inside $\pi$. The connecting line divides $\pi$ (and the whole $E$ ) into two connected components.

Bonds between a cell and a section of the contour of $\Delta$ or between two sections of the contour are defined in a similar way.

Now let $E_{1}$ be a $k$-bond and $E_{2}$ be a $j$-bond between two cells $\Pi_{1}$ and $\Pi_{2}, j \leq k$ and either $E_{1}=E_{2}$ or these bonds are disjoint. If $E_{1}=E_{2}$ then $\Gamma=E_{1}=E_{2}$ is called the $k$-contiguity submap between $\Pi_{1}$ and $\Pi_{2}$ determined by the bond $E_{1}=E_{2}$. If $E_{1}$ and $E_{2}$ are disjoint then the corresponding contiguity submap $\Gamma$ is defined as the smallest submap containing $E_{1}$ and $E_{2}$ and not containing $\Pi_{1}$ and $\Pi_{2}$ (see Figure 5).

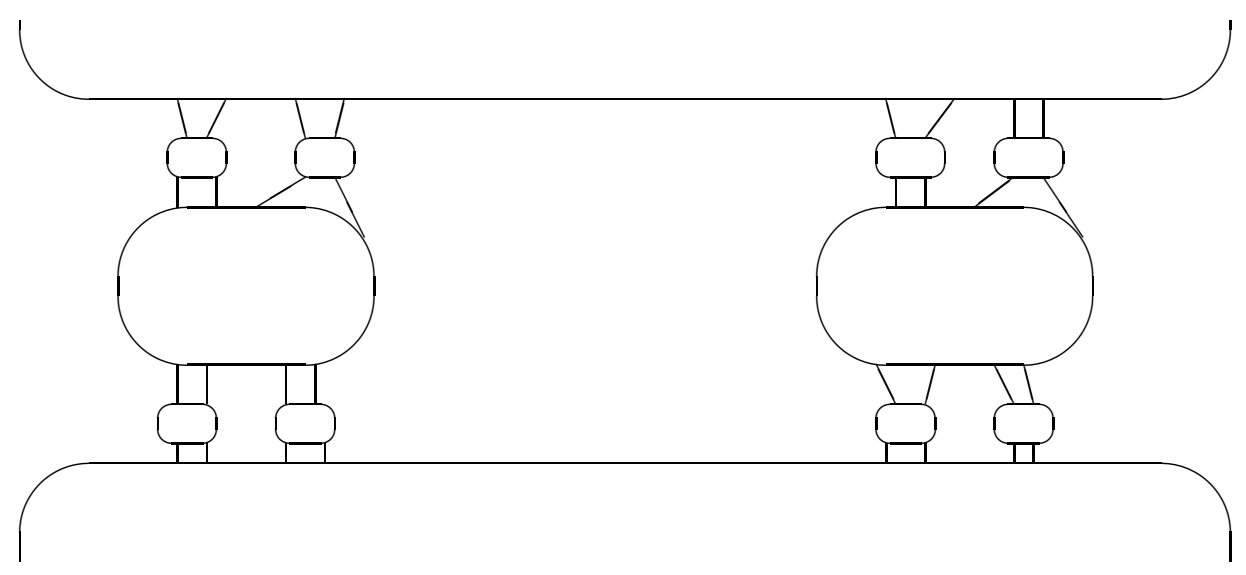

Fig. 5. 
The contiguity arcs $q_{1}$ and $q_{2}$ of $\Gamma$ are intersections of $\partial(\Gamma)$ with $\partial\left(\Pi_{1}\right)$ and $\partial\left(\Pi_{2}\right)$. The contour of $\Gamma$ has the form $p_{1} q_{1} p_{2} q_{2}$ where $p_{1}$ and $p_{2}$ are called the side arcs of $\Gamma$. The connecting line of $\Gamma$ is one of the connecting lines of $E_{1}$ or $E_{2}$. The ratio $\left|q_{1}\right| /\left|\partial\left(\Pi_{1}\right)\right|$ is called the contiguity degree of $\Pi_{1}$ to $\Pi_{2}$ with respect to $\Gamma$ and is denoted by $\left(\Pi_{1}, \Gamma, \Pi_{2}\right)$. If $\Pi_{1}=\Pi_{2}=\Pi$ then $(\Pi, \Gamma, \Pi)$ is a pair of numbers.

Contiguity submaps of a cell to a section of the contour and between sections of the contour are defined in a similar way. (In this paper we do not use notion "degree of contiguity of a section of a contour to" anything.)

We are going to write "bonds" and "contiguity submaps" in place of " $k$-bonds" and " $k$ contiguity submaps". Instead of writing "the contiguity submap $\Gamma$ of a cell $\Pi$ to ...", we sometimes write "the $\Gamma$ - contiguity of $\Pi$ to ...".

The above definition involved the standard decomposition of the contour of a contiguity submap $\Gamma$ into four sections $p_{1} q_{1} p_{2} q_{2}$ where $q_{1}=\Gamma \wedge \Pi_{1}, q_{2}=\Gamma \wedge \Pi_{2}$ (or $q_{2}=\Gamma \wedge q$ if $q$ is a section of $\partial(\Delta))$, we shall write $p_{1} q_{1} p_{2} q_{2}=\partial\left(\Pi_{1}, \Gamma, \Pi_{2}\right)$, and so on.

As in [30] (see §15.1) we fix certain real numbers $\iota<<\zeta<<\varepsilon<<\delta<<\gamma<<\beta<<\alpha$ between 0 and 1 where " $<<$ " means "much smaller". Here "much" means enough to satisfy all the inequalities in Chapters 5, 6 of [30]. We also set $\bar{\alpha}=\frac{1}{2}+\alpha, \bar{\beta}=1-\beta, \bar{\gamma}=1-\gamma, h=\delta^{-1}, n=\iota^{-1}$.

\subsection{Condition A}

The set of positive cells of a diagram $\Delta$ is denoted by $\Delta(2)$ and as before the length of a path in $\Delta$ is the number of positive edges in the path. A path $p$ in $\Delta$ is called geodesic if $|p| \leq\left|p^{\prime}\right|$ for any path $p^{\prime}$ combinatorially homotopic to $p$.

The condition A has three parts:

A1 If $\Pi$ is a cell of rank $j>0$, then $|\partial(\Pi)| \geq n j$.

A2 Any subpath of length $\leq \max (j, 2)$ of the contour of an arbitrary cell of rank $j$ in $\Delta(2)$ is geodesic in $\Delta$.

A3 If $\pi, \Pi \in \Delta(2)$ and $\Gamma$ is a contiguity submap of $\pi$ to $\Pi$ with $(\pi, \Gamma, \Pi) \geq \varepsilon$, then $|\Gamma \wedge \Pi|<$ $(1+\gamma) k$ where $k=r(\Pi)$.

A map satisfying conditions A1, A2, A3 will be called an A-map. As in [30], Section 15.2, a (cyclic) section $q$ of a contour of a map $\Delta$ is called a smooth section of rank $k>0$ (we write $r(q)=k)$ if:

1) every subpath of $q$ of length $\leq \max (k, 2)$ of $q$ is geodesic in $\Delta$;

2 ) for each contiguity submap $\Gamma$ of a cell $\pi$ to $q$ satisfying $(\pi, \Gamma, q) \geq \varepsilon$, we have $|\Gamma \wedge q|<(1+\gamma) k$.

It is shown in [30], $\S 16,17$, that $A$-maps have several "hyperbolic" properties. We summarize these properties here. (They show that $A$-maps of rank $i$ are hyperbolic spaces with hyperbolic constant depending on $i$ only.)

The next three lemmas are Lemmas 15.3, 15.4 and 15.8 in [30].

Lemma 2.1 shows that in a contiguity diagram between two cells side arcs are "much shorter" than perimeters of these cells (so the cells are close to each other).

Lemma 2.1. Let $\Delta$ be an A-map, $\Gamma$ a contiguity submap of either:

(i) a cell $\Pi_{1}$ to a cell $\Pi_{2}$ or 
(ii) a cell $\Pi_{1}$ to a boundary section $q^{1}$ of $\Delta$, or

(iii) a boundary section $q^{1}$ to a boundary section $q^{2}$.

Let $p_{1}$ and $p_{2}$ be the side arcs of $\Gamma$, and $c=\max \left(\left|p_{1}\right|,\left|p_{2}\right|\right)$. Then $c<2 \varepsilon^{-1} r\left(\Pi_{1}\right)<\zeta n r\left(\Pi_{1}\right)$ in cases (i) and (ii), and $c<\zeta n r\left(\Pi_{2}\right)$ in case (i). Furthermore $c<\zeta n r\left(q^{1}\right)$ in cases (ii) and (iii) provided $q^{1}$ is smooth.

Lemma 2.2 says that contiguity arcs in a contiguity subdiagram have "almost the same lengths".

Lemma 2.2. Let $\psi$ be the degree of $\Gamma$-contiguity of a cell $\Pi_{1}$ to a cell $\Pi_{2}$ (or to a section $q$ of a contour) in an $A$-map $\Delta$, and $q_{1}=\Gamma \wedge \Pi_{1}, q_{2}$ are the contiguity arcs of $\Gamma$. Then

$$
\left(\bar{\beta}-2 \zeta \psi^{-1}\right)\left|q_{1}\right|<\left|q_{2}\right| .
$$

In particular, $\left|q_{2}\right|>(\psi-2 \beta)\left|\partial\left(\Pi_{1}\right)\right|$, and if also $\psi \geq \varepsilon$, then $\left|q_{1}\right|<(1+2 \beta)\left|q_{2}\right|$.

Furthermore if $q_{2}=\Gamma \wedge \Pi_{2}$ or $q_{2}=\Gamma \wedge q$ and $q$ is a smooth section, then

$$
\left|q_{1}\right|>\bar{\beta}\left(1+2 \zeta \psi^{-1}\right)^{-1}\left|q_{2}\right| .
$$

In particular, if $\psi \geq \varepsilon$, then

$$
\left|q_{1}\right|>(1-2 \beta)\left|q_{2}\right| \text {. }
$$

Lemma 2.3 shows that the degree of contiguity of one cell to another cell cannot be significantly bigger than $1 / 2$ and the degree of contiguity of a cell of a bigger rank to a cell of a smaller rank is very small.

Lemma 2.3. In an arbitrary A-map $\Delta$ the degree of contiguity of an arbitrary cell $\pi$ to an arbitrary cell $\Pi$ or to an arbitrary smooth section $q$ of the contour via an arbitrary contiguity submap $\Gamma$ is less than $\bar{\alpha}$, and the degree of contiguity of $\pi$ to $\Pi$ (or to q) with $r(\Pi) \leq r(\pi) \quad($ or $r(q) \leq r(\pi))$ is less than $\varepsilon$.

The next lemma is a generalization of the Greenlinger lemma from the theory of small cancellation groups [23]. It shows that any $A$-map contains a cell whose boundary is almost entirely contiguous to the boundary of the diagram.

Lemma 2.4. a) Let $\Delta$ be a disc A-map whose contour is subdivided into at most 4 sections $q^{1}, q^{2}, q^{3}, q^{4}$, and $r(\Delta)>0$. Then there exists a positive cell $\pi$ and disjoint contiguity submaps $\Gamma_{1}, \ldots, \Gamma_{4}$ of $\pi$ to $q^{1}, \ldots, q^{4}$ (some of these submaps may be absent) such that the sum of corresponding contiguity degrees $\sum_{i=1}^{4}\left(\pi, \Gamma_{i}, q^{i}\right)$ is greater than $\bar{\gamma}=1-\gamma$.

b) Let $\Delta$ be an annular $A$-map whose contours are subdivided into at most 4 sections $q^{1}, q^{2}, q^{3}, q^{4}$ regarded as cyclic or ordinary paths, and $r(\Delta)>0$. Then $\Delta$ has a positive cell $\pi$ and disjoint contiguity submaps $\Gamma_{1}, \ldots, \Gamma_{4}$ of $\pi$ to $q^{1} q^{2}, q^{3}, q^{4}$ (up to three of these submaps may be absent) such that the sum of contiguity degrees of these contiguity submaps is greater than $\bar{\gamma}$.

Proof. Both statements are immediate corollaries of Theorem 16.1 from [30].

The next lemma follows from Lemma 2.4 in the same way as Theorem 16.2 follows from Corollary 16.1 in $[30]$. 
Lemma 2.5. If $\Delta$ is a disc or annular $A$-map with contour subdivided into at most 4 sections, and $r(\Delta)>0$, then there exists a positive cell $\Pi$ and a contiguity submap $\Gamma$ of $\Pi$ to one of the sections $q$ of the contour of $\Delta$ such that $r(\Gamma)=0$ and $(\Pi, \Gamma, q) \geq \varepsilon$.

Here we add an additional property whose proof is contained in the proof of Theorem 16.2 in [30].

Lemma 2.6. Let $\Delta$ be a disc or annular A-map with contour subdivided into at most 4 sections. Assume there is a cell $\Pi$ in $\Delta$ and a contiguity submap $\Gamma$ of $\Pi$ to one of the sections $q$ of the boundary with $(\Pi, \Gamma, q) \geq \varepsilon$. Then there is a cell $\pi$ of $\Delta$ and a contiguity submap $\Gamma^{\prime}$ of $\pi$ to $q$ such that $r\left(\Gamma^{\prime}\right)=0$ and $\left(\pi, \Gamma^{\prime}, q\right) \geq \varepsilon$.

The next four lemmas are Theorem 17.1, Corollary 17.1, Lemma 17.1 and Lemma 17.2 in [30]. The following lemma shows that smooth sections of contours of $A$-maps are almost geodesic.

Lemma 2.7. Let $\Delta$ be a disc map with contour $q$ t or an annular $A$-map with contours $q$ and $t$. If $q$ is a smooth section then $\bar{\beta}|q| \leq|t|$.

The next nine lemmas demonstrate different variants of the fact that in $A$-maps, quasigeodesic quadrangles are thin. But we need more precise statements than in the usual theory of hyperbolic spaces. The constants appearing in these statements do not depend on the rank of diagrams while the hyperbolic constants of $A$-maps tends to infinity with the rank.

Lemma 2.8. If a disc A-map $\Delta$ contains a cell $\Pi$ of positive rank, then $|\partial(\Delta)|>\bar{\beta}|\partial(\Pi)|$.

Lemma 2.9. Let $\Delta$ be a disc A-map with contour $p_{1} q_{1} p_{2} q_{2}$ where $q_{1}$ and $q_{2}$ are smooth sections such that $\left|q_{1}\right|+\left|q_{2}\right|>0,\left|p_{1}\right|+\left|p_{2}\right| \leq \gamma\left(\left|q_{1}\right|+\left|q_{2}\right|\right)$. Then there is either a 0-bond between $q_{1}$ and $q_{2}$ or a cell $\Pi \in \Delta(2)$ and disjoint contiguity submaps $\Gamma_{1}$ and $\Gamma_{2}$ of $\Pi$ to $q_{1}$ and $q_{2}$, respectively, such that $\left(\Pi, \Gamma_{1}, q_{1}\right)+\left(\Pi, \Gamma_{2}, q_{2}\right)>\bar{\beta}=1-\beta$.

We also need the following version of Lemma 2.9.

We say that a bond between two boundary sections $q_{1}$ and $q_{2}$ of a map is narrow if it is either a 0-bond or, in the inductive definition of the bond, we add that $\left(\pi, \Gamma_{1}, q_{1}\right)+\left(\pi, \Gamma_{2}, q_{2}\right)>1-\alpha / 2$ for the principal cell $\pi$ of the bond. A contiguity submap between $q_{1}$ and $q_{2}$ is narrow if it is defined by narrow bonds (according to the inductive definition).

Lemma 2.10. Let $\Delta$ be a disc $A$-map with contour $p_{1} q_{1} p_{2} q_{2}$ where $q_{1}$ and $q_{2}$ are smooth sections such that $\left|q_{1}\right|+\left|q_{2}\right|>0,\left|p_{1}\right|+\left|p_{2}\right| \leq \alpha^{2}\left(\left|q_{1}\right|+\left|q_{2}\right|\right)$. Then there is either a 0 -bond between $q_{1}$ and $q_{2}$ or a cell $\Pi \in \Delta(2)$ and disjoint contiguity submaps $\Gamma_{1}$ and $\Gamma_{2}$ of $\Pi$ to $q_{1}, q_{2}$ (respectively) such that $\left(\Pi, \Gamma_{1}, q_{1}\right)+\left(\Pi, \Gamma_{2}, q_{2}\right) \geq 1-\alpha / 2$. Hence $\Pi, \Gamma_{1}$ and $\Gamma_{2}$ form a narrow bond between $q_{1}$ and $q_{2}$.

Proof. The difference between the formulations of Lemma 2.10 and Lemma 17.2 of [30] is that the parameters $\gamma$ and $\bar{\beta}$ are now substituted by $\alpha^{2}$ and $1-\alpha / 2$, respectively. The proof differs from the proof of Lemma 17.2 [30] only in some insignificant details.

There is no change in Case 1) of the proof of Lemma 17.2 [30]. The only alteration in Case 2) is the substitution of the first factor $\gamma$ for $\alpha^{2}$ in the left-hand side of the long inequality at the end. It is easy to see that the resulting inequality is also true by the choice of the parameters.

In Case 3), we should assume now that $\left(\Pi, \Gamma, p_{1}\right)>\bar{\gamma}-(1-\alpha / 2)=\alpha / 2-\gamma$ (instead of $\left(\Pi, \Gamma, p_{1}\right)>\bar{\gamma}-\bar{\beta}=\beta-\gamma$ in [30]). This leads to the similar substitution of the term $\beta-\gamma$ for $\alpha / 2-\gamma$ in the right-hand side of inequality (7) of the proof. It remains to notice that the last long 
inequality of Case 3) is still true after the substitution of $\beta-\gamma$ by $\alpha / 2-\gamma$ in the right-hand side, and the substitution of the factor $\gamma$ by $\alpha^{2}$ in the left-hand side.

The difference $\beta-\gamma$ should be replaced by $\alpha / 2-\gamma$ in the hypothesis of Case 4$)$. This leads to the same substitution in inequality (9) of the proof of Lemma 17.2 [30]. Then again, it remains to replace the term $\beta-\gamma$ by $\alpha / 2-\gamma$ in the right-hand side of the concluding inequality, and to replace the factor $\gamma$ by $\alpha^{2}$ in the left-hand side of it.

Lemma 2.11. Let $\Delta$ be a disc A-map with contour $p_{1} q_{1} p_{2} q_{2}$, where $q_{1}$ and $q_{2}$ are smooth sections of some ranks $k$ and $l, k \leq l$, and $\left|p_{1}\right|+\left|p_{2}\right| \leq \gamma\left(\left|q_{1}\right|+\left|q_{2}\right|\right)$. Then there is a narrow contiguity submap between $q_{1}$ and $q_{2}$ with contour $p_{1}^{\prime} q_{1}^{\prime} p_{2}^{\prime} q_{2}^{\prime}$, where $q_{1}=u^{\prime} q_{1}^{\prime} u^{\prime \prime}, q_{2}=v^{\prime \prime} q_{2}^{\prime} v^{\prime}$ and $\left|u^{\prime}\right|,\left|v^{\prime}\right|<$ $\gamma^{-1}\left(\left|p_{1}\right|+\alpha k\right),\left|u^{\prime \prime}\right|,\left|v^{\prime \prime}\right|<\gamma^{-1}\left(\left|p_{2}\right|+\alpha k\right)$.

Proof. Up to the term "narrow", the proof is the same as the proof of items 1) and 2) of Lemma 17.3 [30] (Lemma 2.12 below). In fact Lemma 2.11 follows from the proof of Lemma 17.3 [30] because $\alpha / 2>\beta$.

Lemma 2.12. (Lemma 17.3 [30]) Let $\Delta$ be a disc A-map with contour $p_{1} q_{1} p_{2} q_{2}$ where $q_{1}$ and $q_{2}$ are smooth sections of ranks $k$ and $l, k \leq l$, such that

$$
\left|p_{1}\right|+\left|p_{2}\right| \leq \gamma\left(\left|q_{1}\right|+\left|q_{2}\right|\right)
$$

Then:

(1) there exist vertices $o_{1}$ and $o_{2}$ on $q_{1}$ and $q_{2}$ and a path $x$ joining them in $\Delta$ such that $|x|<\alpha k$.

(2) one can choose $o_{1}$ and $o_{2}$ in such a way that the lengths of the initial segments of $q_{1}$ and $q_{2}^{-1}$ (or of $q_{1}^{-1}$ and $q_{2}$ ) ending at $o_{1}$ and $o_{2}$, are less than $\gamma^{-1}\left(\left|p_{1}\right|+\alpha k\right)$ (or than $\gamma^{-1}\left(\left|p_{2}\right|+\alpha k\right)$ ). (3) if (9) is replaced by

$$
\left|p_{1}\right|,\left|p_{2}\right|<\alpha k
$$

then any vertex o of $q_{1}$ (or of $q_{2}$ ) can be joined in $\Delta$ by a path $y$ to a vertex $o^{\prime}$ on $q_{2}$ (or on $q_{1}$ ) in such a way that $|y|<\gamma^{-1} k$.

Consider an A-map $\Delta$ with contour $p_{1} q_{1} p_{2} q_{2}$, where $q_{1}, q_{2}$ are smooth sections and $\Delta$ is a narrow contiguity submap between $q_{1}$ and $q_{2}$. We define an $\alpha$-series as a maximal series of disjoint narrow bonds between $q_{1}$ and $q_{2}$. In particular, the maximal series contains the bonds from the definition of narrow contiguity submap. By $\Pi_{0}$ we denote a cell of maximal perimeter among principal cells of the bonds over all $\alpha$-series.

Lemma 2.13. With the preceding notation, any vertex of $q_{1}$ is at the distance at most $\alpha^{-1}\left|\partial\left(\Pi_{0}\right)\right|$ from the path $q_{2}$.

Proof. Assume that $E_{1}, \ldots, E_{s}$ is the $\alpha$-series. Let $x_{1}^{l} y_{1}^{l} x_{2}^{l} y_{2}^{l}$ be the standard contour of $E_{l}$, where $y_{j}^{l}$ is a subpath of $q_{j}$ for $j=1,2$. By Lemma 2.1 and the definition of narrow bonds, $\left|x_{j}^{l}\right|<$ $(\alpha+2 \zeta)\left|\partial\left(\Pi_{0}\right)\right|$. By Lemmas 2.2 and 2.3, $\left|y_{j}^{l}\right|<(1-2 \beta)^{-1} \bar{\alpha}\left|\partial\left(\Pi_{0}\right)\right|$. Therefore the distance between any vertex of some $y_{1}^{l}$ and $q_{2}$ is less than $\left(\alpha+2 \zeta+(1-2 \beta)^{-1} \bar{\alpha} / 2\right)\left|\partial\left(\Pi_{0}\right)\right|<\left|\partial\left(\Pi_{0}\right)\right| / 3<\alpha^{-1}\left|\partial\left(\Pi_{0}\right)\right|$.

Then we consider the submaps $\Delta_{1}, \ldots, \Delta_{s-1}$ with contours $\left(x_{2}^{l}\right)^{-1} z_{1}^{l}\left(x_{1}^{l+1}\right)^{-1} z_{2}^{l}$, where $z_{j}^{l}$ is a subpath of $q_{j}$ for $j=1,2$. One may suppose that $\left|z_{1}^{l}\right|+\left|z_{2}^{l}\right|<\alpha^{-2}\left(\left|x_{2}^{l}\right|+\left|x_{1}^{l+1}\right|\right)$, because otherwise by Lemma 2.10 there would be an additional narrow bond in $\Delta_{l}$, which contradicts the definition of the $\alpha$-series. (Indeed $\left(\Pi, \Gamma_{j}, q_{j}\right) \geq 1-\alpha / 2-\bar{\alpha}>\varepsilon$ for $j=1,2$ in the Lemma 2.10 by 
Lemma 2.3, and therefore that cell $\Pi$ is a principle cell of a narrow bond between $q_{1}$ and $q_{2}$.) Since $\left|x_{2}^{l}\right|+\left|x_{1}^{l+1}\right|<(\alpha+4 \zeta)\left|\partial\left(\Pi_{0}\right)\right|$, we have $\left|z_{1}^{l}\right|<\alpha^{-1}\left(1+4 \zeta \alpha^{-1}\right)\left|\partial\left(\Pi_{0}\right)\right|$, and the distance between any vertex of $z_{1}^{l}$ and $q_{2}$ is less than $\left|z_{1}^{l}\right| / 2+(\alpha+2 \zeta)\left|\partial\left(\Pi_{0}\right)\right|<\alpha^{-1}\left|\partial\left(\Pi_{0}\right)\right|$, and the lemma is proved.

Lemma 2.14. (Lemma 17.4 [30].) Let $\Delta$ be a disc A-map with contour $p_{1} q_{1} p_{2} q_{2}$, where $q_{1}$ and $q_{2}$ are smooth sections of ranks $k$ and $l, k \leq l$, and $\left|p_{1}\right|,\left|p_{2}\right|<\zeta n k$. Then the perimeter of every cell of $\Delta$ is less than $3 \gamma^{-1} \zeta n k<n k$ and $r(\Delta)<3 \gamma^{-1} \zeta k<k$.

Lemma 2.15. (Lemma 17.5 [30]). Let $\Delta$ be a disc A-map with contour $p_{1} q_{1} p_{2} q_{2}$, where $q_{1}$ and $q_{2}$ are smooth sections of ranks $k$ and $l, k \leq l$, and $\left|q_{j}\right|-M \geq 0$ for $j=1,2$ and $M=\gamma^{-1}\left(\left|p_{1}\right|+\left|p_{2}\right|+k\right)$. Then it is possible to excise a submap from $\Delta$ with contour $p_{1}^{\prime} q_{1}^{\prime} p_{2}^{\prime} q_{2}^{\prime}$ where $q_{1}^{\prime}\left(q_{2}^{\prime}\right)$ is a subpath of $q_{1}$ (of $q_{2}$ ), $\left|p_{1}^{\prime}\right|,\left|p_{2}^{\prime}\right|<\alpha k$, and $\left|q_{j}^{\prime}\right|>\left|q_{j}\right|-M$ for $j=1,2$.

The next lemma shows that annular $A$-maps can be cut by short paths to obtain a disc map.

Lemma 2.16. (Lemma 17.1, [30]) Let $\Delta$ be an annular A-map with contours $p$ and $q$ such that any loop consisting of 0 -edges of $\Delta$ is 0 -homotopic. Then there is a path $t$ connecting some vertices $o_{1}$ and $o_{2}$ of the paths $p$ and $q$ respectively, such that $|t|<\gamma(|p|+|q|)$.

\section{Burnside quotients}

\subsection{Axioms}

Recall that in Chapter 6 of [30], it is proved that for any sufficiently large odd $n$, there exists a presentation of the free Burnside group $B(m, n)$ with $m \geq 2$ generators, such that every reduced diagram over this presentation is an $A$-map.

Our goal here is to generalize the theory from [30] to Burnside factors of non-free groups in the following way.

In [30], 0-edges are labeled by 1 only, and (trivial) 0 -relators have the form $1 \cdot \ldots \cdot 1$ or $a \cdot 1 \cdot \ldots \cdot 1$. $a^{-1} \cdot 1 \cdot \ldots \cdot 1$. We need to increase the collection of labels of 0 -edges and the collection of 0 -relations. In other words, we divide the generating set of our group into the set of 0-letters and the set of positive letters. Then in the diagrams, 0-letters label 0-edges. We shall also add defining relators of our group which have the form $u a v b^{-1}$ where $u, v$ are words consisting of 0-letters only (we call such words 0 -words), and $a, b$ are a positive letters, as well as defining relations without positive letters, to the list of zero relations. The corresponding cells in diagrams will be called 0-cells.

Let $\mathbf{G}$ be a group given by a presentation $\langle\mathcal{X} \mid \mathcal{R}\rangle$. Let $\mathcal{X}$ be a symmetric set (closed under taking inverses). Let $\mathcal{Y} \subseteq \mathcal{X}$ be a symmetric subset which we shall call the set of non-zero letters or $Y$-letters. All other letters in $\mathcal{X}$ will be called 0-letters.

By the $Y$-length of a word $A$, written $|A|_{Y}$ or just $|A|$, we mean the number of occurrences of $Y$-letters in $A$. The $Y$-length $|g|_{Y}$ of an element $g \in G$ is the length of the $Y$-shortest word representing element $g$.

Let $\mathbf{G}(\infty)$ be the group given by the presentation $\langle\mathcal{X} \mid \mathcal{R}\rangle$ inside the variety of Burnside groups of exponent $n$. We will choose a presentation of the group $\mathbf{G}(\infty)$ in the class of all groups, that is we add enough relations of the form $A^{n}=1$ to ensure that $\mathbf{G}(\infty)$ has exponent $n$. This infinite presentation will have the form $\left\langle\mathcal{X} \mid \mathcal{R}(\infty)=\cup \mathcal{S}_{i}\right\rangle$ where sets $\mathcal{S}_{i}$ will be disjoint, and relations from $\mathcal{S}_{i}$ will be called relations of rank $i$. Unlike [30, Chapter 6], $i$ runs over $0,1 / 2,1,2,3, \ldots$ $\left(i=0,1,2, \ldots\right.$ in $\left[30\right.$, Chapter 6].) The set $\mathcal{R}$ will be equal to $\mathcal{S}_{0} \cup \mathcal{S}_{1 / 2}$. The detailed description 
of sets $\mathcal{S}_{i}$ will be given below. We shall call this presentation of $\mathbf{G}(\infty)$ a graded presentation. Our goal is the same as in [30]: we want every diagram over $\mathcal{R}(\infty)$ to be an A-map.

We are going to prove that this goal is possible to achieve if $\mathcal{R}$ satisfies conditions (Z1), (Z2), (Z3) presented below. While listing these conditions, we also fix some notation and definitions.

(Z1) The set $\mathcal{R}$ is the union of two disjoint subsets $\mathcal{S}_{0}=\mathcal{R}_{0}$ and $\mathcal{S}_{1 / 2}$. The group $\left\langle\mathcal{X} \mid \mathcal{S}_{0}\right\rangle$ is denoted by $\mathbf{G}(0)$ and is called the group of rank 0 . We call relations from $\mathcal{S}_{0}$ relations of rank 0 . The relations from $\mathcal{S}_{1 / 2}$ have rank $1 / 2$. The group $\langle\mathcal{X} \mid \mathcal{R}\rangle$ is denoted by $\mathbf{G}(1 / 2)$.

(Z1.1) The set $\mathcal{S}_{0}$ consists of all relations from $\mathcal{R}$ which have $Y$-length 0 and all relations of $\mathcal{R}$ which have the form (up to a cyclic shift) $a y_{1} b y_{2}^{-1}=1$ where $y_{1}, y_{2} \in Y^{+}, a$ and $b$ are of $Y$-length 0 (i.e. 0 -words).

(Z1.2) The set $\mathcal{S}_{0}$ implies all Burnside relations $u^{n}=1$ of $Y$-length 0.

The subgroup of $\mathbf{G}(0)$ generated by all 0-letters will be called the 0-subgroup of $\mathbf{G}(0)$. Elements from this subgroup are called 0-elements. Elements which are not conjugates of elements from the 0 -subgroup will be called essential. An essential element $g$ from $\mathbf{G}(0)$ is called cyclically $Y$-reduced if $\left|g^{2}\right|_{Y}=2|g|_{Y}$. We shall show that $g$ is cyclically $Y$-reduced if and only if it is cyclically minimal (in rank 0), i.e. it is not a conjugate of a $Y$-shorter element in $\mathbf{G}(0)$ (Lemma 3.5).

Notice that condition (Z1.1) allows us to consider $Y$-bands in van Kampen diagrams over $\mathcal{S}_{0}$. Maximal $Y$-bands do not intersect.

(Z2) The relators of the set $\mathcal{S}_{1 / 2}$, will be called hubs. The corresponding cells in van Kampen diagrams are also called hubs. They satisfy the following properties

(Z2.1) The $Y$-length of every hub is at least $n$.

(Z2.2) Every hub is linear in $Y$, i.e. contains at most one occurrence $y^{ \pm 1}$ for every letter $y \in Y$.

(Z2.3) Assume that each of words $v_{1} w_{1}$ and $v_{2} w_{2}$ is a cyclic permutation of a hub or of its inverse, and $\left|v_{1}\right| \geq \varepsilon\left|v_{1} w_{1}\right|$. Then an equality $u_{1} v_{1}=v_{2} u_{2}$ for some 0 -words $u_{1}, u_{2}$, implies in $\mathbf{G}(0)$ equality $u_{2} w_{1}=w_{2} u_{1}$ (see Figure 6).

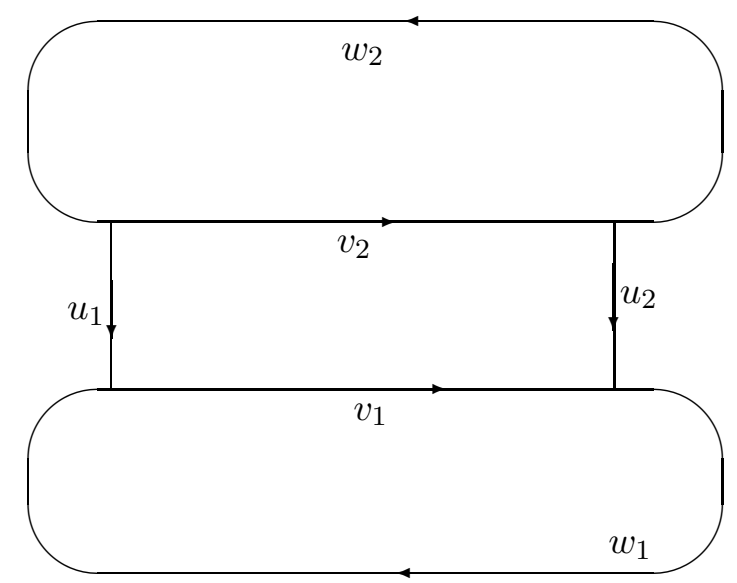

Fig. 6. 
A word $B$ over $\mathcal{X}$ is said to be cyclically minimal in rank $1 / 2$ if it is not a conjugate in $\mathbf{G}(1 / 2)$ of a word of smaller $Y$-length. An element $g$ of $\mathbf{G}(0)$ is called cyclically minimal in rank $1 / 2$ if it is represented by a word which is cyclically minimal in rank $1 / 2$.

(Z3) With every essential element $g \in \mathbf{G}(0)$ we associate a subgroup $\mathbf{0}(g) \leq \mathbf{G}(0)$, normalized by $g$. If $g$ is cyclically $Y$-reduced, let $\mathbf{0}(g)$ be the maximal subgroup consisting of 0-elements which contains $g$ in its normalizer. By Lemmas 3.3 (2) and 3.4 (3) below arbitrary essential element $g$ is equal to a product $v u v^{-1}$ where $u$ is cyclically $Y$-reduced. In this case we define $\mathbf{0}(g)=v \mathbf{0}(u) v^{-1}$. We shall prove in Lemma 3.7 that $\mathbf{0}(g)$ is well defined.

(Z3.1) Assume that $g$ is an essential cyclically minimal in rank $1 / 2$ element of $\mathbf{G}(0)$ and for some $x \in \mathbf{G}(0)$, both $x$ and $g^{-4} x g^{4}$ are 0-elements. Then $x \in \mathbf{0}(g)$.

(Z3.2) For every essential cyclically minimal in rank $1 / 2$ element $g \in \mathbf{G}(0)$ there exists a 0 element $r$ such that $g r^{-1}$ commutes with every element of $\mathbf{0}(g){ }^{7}$

In this section, we show how the theory from [30, Chapter 6] can be adapted to diagrams over a presentation satisfying (Z1), (Z2), (Z3).

\subsection{Corollaries of (Z1), (Z2), (Z3)}

Here we are going to deduce some useful facts about groups $\mathbf{G}(0)$ whose presentations satisfy (Z1), (Z2), (Z3). (Some of them can be deduced from a presentation of $\mathbf{G}(0)$ as an HNN-extension.)

The following lemma is obvious.

Lemma 3.1. If the set $\mathcal{R}$ satisfies property (Z1.1) then there exists a homomorphism from $\mathbf{G}(0)$ to the group of integers $\mathbf{Z}$ which sends every $Y$-letter to 1 and every 0 -letter to 0.

Lemma 3.2. Suppose that $\mathcal{R}$ satisfies (Z1.1). Let $\Delta$ be a van Kampen diagram over $\mathcal{S}_{0}$ and $p$ be a subpath of the boundary $\partial(\Delta)$. Suppose that a $Y$-band starting on $p$ ends on $p^{-1}$. Then $\phi(p)$ contains a subword of $Y$-length 2 which is equal to a 0 -word in $\mathbf{G}(0)$.

Proof. Since maximal $Y$-bands cannot intersect, there exists a $Y$-band $\mathcal{T}$ starting on edge $e$ and ending on edge $f^{-1}$, where $e, f \in p$, such that the shortest subpath $p_{1}$ of $p$ containing $e$ and $f$ has length 2. Let $\Delta_{1}$ be the subdiagram of $\Delta$ bounded by $p_{1}$ and one of the sides $q_{1}$ of $\mathcal{T}$. The word $\phi\left(q_{1}\right)$ is a 0 -word, so $\phi\left(p_{1}\right)$ is a subword of $A$ of $Y$-length 2 which is equal to a 0 -word in $\mathbf{G}(0)$.

A word $A$ which does not contain a subword of $Y$-length 2 that is equal to a 0 -word in $\mathbf{G}(0)$, will be called $Y$-reduced. A word $A$ is called cyclically $Y$-reduced if every cyclic shift of $A$ is $Y$-reduced.

Lemma 3.3. Suppose that $\mathcal{R}$ satisfies (Z1.1). Then the following conditions hold.

(1) Let $A$ be a word representing an element $g \in \mathbf{G}(0)$, but $|A|_{Y}>|g|_{Y}$. Then $A$ is not $Y$-reduced.

\footnotetext{
${ }^{7}$ At the beginning of May 2002, influenced by the talk by Sergei Ivanov at the Geometric Group Theory conference in Montreal, we realized that Condition (Z3.2) can be replaced by the following weaker condition:

(Z3.2') For every essential cyclicly minimal in rank $1 / 2$ element $g \in \mathbf{G}(0)$, the extension of $\mathbf{0}(g)$ by the automorphism induced by $g$ (acting by conjugation) satisfies the identity $x^{n}=1$.

It is clear, that in the proof of Lemma 3.12 below (the only lemma in this paper, where (Z3.2) is used), property (Z3.2) can be replaced by (Z3.2').
} 
(2) If $A$ is a $Y$-reduced word that represents a conjugate of an essential element $g$, but it is not a shortest word representing a conjugate of $g$, then the word $A$ has the form as a product $U V U^{\prime}$ where $|U|_{Y}>0$ and $U^{\prime} U$ is equal to a 0 -word in $\mathbf{G}(0), V$ is a cyclically $Y$-reduced word.

Proof. (1) Let $B$ be a $Y$-shortest word representing $g$. Then there exists a diagram over $\mathcal{S}_{0}$ with boundary $p q$ where $\phi(p) \equiv A, \phi(q) \equiv B^{-1}$.

Condition (Z1.1) imply that every $Y$-edge on $A$ is the start edge of a maximal $Y$-band. Each of these $Y$-bands ends on the boundary of $\Delta$. Since $|A|_{Y}>|B|_{Y}$, one of the $Y$-bands starting on $p$ ends on $p$. By Lemma 3.2 there exists a subword of $A$ of length 2 which is equal to a 0 -word in $\mathbf{G}(0)$.

To prove part (2), one can take an annular diagram instead of a disc diagram as in part (1), and prove that a cyclic shift of $A$ has a subword of $Y$-length 2 which is equal to a 0 -word in $\mathbf{G}(0)$. Hence $A \equiv U V U^{\prime}$ where $|U|_{Y}>0, U^{\prime} U$ is equal to a 0 -word $W$ in $\mathbf{G}(0)$. Since the word $V W$ represents a conjugate of $g$ and is shorter than $A$, we can conclude the proof by induction on $|A|_{Y}$.

Lemma 3.4. Suppose that $\mathcal{R}$ satisfies (Z1.1). Then the following statements are true.

(1) If $g \in \mathbf{G}(0)$ and $A$ is a $Y$-reduced word representing $g$ then there exists a decomposition $A \equiv A_{1} A_{2}$ such that the element represented by $A_{2} A_{1}$ is cyclically $Y$-reduced in $\mathbf{G}(0)$.

(2) If $g \in \mathbf{G}(0)$ is cyclically $Y$-reduced then for every integer $m,\left|g^{m}\right|_{Y}=\left.|m|\right|_{Y}$ and $g^{m}$ is cyclically $Y$-reduced.

(3) If $g$ is cyclically $Y$-reduced and $A$ is a $Y$-shortest word representing $g$ in $\mathbf{G}(0)$ then $A$ is cyclically $Y$-reduced.

Proof. (1) By Lemma 3.3 (1) we can suppose that $A$ is a $Y$-shortest word representing $g$. Let $B$ be a shortest word representing $g^{2}$. If $|B|_{Y}=2|A|_{Y}$ then $g$ is cyclically $Y$-reduced. Suppose that $2|A|_{Y}>|B|_{Y}$. By Lemma 3.3 there exists a subword $C$ of $A^{2}$ of $Y$-length 2 which is equal to a 0 -word $D$ in $\mathbf{G}(0)$. By Lemma 3.1 we can assume that $C \equiv y_{1} u y_{2}^{-1}$ (or $C \equiv y_{1}^{-1} u y_{2}$ ) where $u$ is a 0 -word and $y_{1}, y_{2} \in Y^{+}$.

Since $A$ is $Y$-reduced, one can represent $A$ as $u_{2} y_{2}^{-1} A_{1} y_{1} u_{1}$ where $u_{1} u_{2} \equiv u$. Then the cyclic shift $A_{1} y_{1} u y_{2}$ of $A$ can be represented by $A_{1} D$ which is a reduced word. Since this word is $Y$-shorter than $A$ we can apply induction and conclude that there exists a cyclic shift of $A_{1} D$ which represents a cyclically reduced element.

(2) Let $A$ be a shortest word representing $g$. Suppose that for some $m>0, A^{m}$ is not a shortest word representing $g^{m}$. Then by Lemma $3.3 A^{m}$ contains a subword of $Y$-length 2 which is equal to a 0 -word in $\mathbf{G}(0)$. But then the same subword occurs in $A^{2}$, so $A^{2}$ is not a shortest word representing $g^{2}$, a contradiction.

(3) Notice that for every cyclic shift $A_{1}$ of $A, A_{1}^{2}$ is a subword of $A^{3}$. It remains to apply part (2) of this lemma.

Lemma 3.5. Suppose that $\mathcal{R}$ satisfies (Z1.1). Let $A$ be a $Y$-shortest word representing an essential element $g$ in $\mathbf{G}(0)$.

Then the following conditions are equivalent:

(i) A is cyclically $Y$-reduced; 
(ii) $g$ is cyclically minimal in $\mathbf{G}(0)$ (that is $g$ is not conjugate in $\mathbf{G}(0)$ of an element of a smaller $Y$-length);

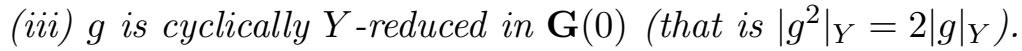

Proof. (i) $\rightarrow$ (ii). Suppose that every cyclic shift of $A$ is $Y$-reduced. Then $A$ cannot be represented in the form $U V U^{\prime}$ where $|U|_{Y}>0$ and $U^{\prime} U$ is equal to a 0 -word in $\mathbf{G}(0)$. Therefore by Lemma 3.3 (2), $A$ is a shortest word among all words representing conjugates of $g$. Hence $g$ is not a conjugate of a shorter word, so $g$ is cyclically minimal.

(ii) $\rightarrow$ (iii). Suppose that $g$ is cyclically minimal but $\left|g^{2}\right|_{Y}<2|g|_{Y}$. Then $A^{2}$ is not a shortest word representing $g^{2}$. By Lemma $3.3(1), A^{2}$ is not $Y$-reduced, which means that $A^{2}$ contains a subword $W$ of $Y$-length 2 which is equal to a 0 -word in $\mathbf{G}(0)$. Since $A$ is reduced (by assumption it is a shortest word representing $g), W \equiv U^{\prime} U$ where $U^{\prime}$ is a suffix of $A, U$ is a prefix of $A$. By Lemma 3.1, $|A|_{Y} \geq 2$. Hence $A \equiv U V U^{\prime}$ for some word $V$. The cyclic shift $V U^{\prime} U$ of $A$ is not $Y$-reduced, so it represents an element of $\mathbf{G}(0)$ which is shorter than $g$. Hence $g$ is a conjugate of a shorter element, a contradiction.

(iii) $\rightarrow$ (i). This follows directly from Lemma $3.4(3)$.

Lemma 3.6. Suppose that $\mathcal{R}$ satisfies (Z1.1). Let $x$ and $g^{-1} x g$ be 0 -elements and $g=h f$ where $|g|=|f|+|h|$. Then $h^{-1} x h$ is also an 0-element.

Proof. Let $X, G, H$ be $Y$-reduced words representing the elements $x, g, h$, respectively. By Lemma 3.3 , the only possible reduction of the word $G^{-1} X G$ can be fulfilled in the middle of it. Then, after $|h|$ such reductions, we will see that the subword $H X H^{-1}$ is equal to a 0 -word.

The next several lemmas deal with the subgroups $\mathbf{0}(g)$ in property (Z3).

Lemma 3.7. Suppose that $\mathcal{R}$ satisfies (Z1.1). Then:

(1) the subgroup $\mathbf{0}(g)$ is well-defined, i.e. $v \mathbf{0}(u) v^{-1}$ does not depend on the presentation vuv ${ }^{-1}$ of an element $g$ where $u$ is cyclically $y$-reduced.

(2) For every $h \in \mathbf{G}(0)$ and every essential $g$, we have $\mathbf{0}\left(h g h^{-1}\right)=h \mathbf{0}(g) h^{-1}$.

Proof. Assume that $g=v^{\prime} u^{\prime}\left(v^{\prime}\right)^{-1}$ and $v^{\prime} \mathbf{0}\left(u^{\prime}\right)\left(v^{\prime}\right)^{-1} \neq v \mathbf{0}(u) v^{-1}$. Then

$$
\mathbf{0}\left(u^{\prime}\right) \neq\left(v^{\prime}\right)^{-1} v \mathbf{0}(u) v^{-1} v^{\prime}
$$

Thus, to prove the statement, one may assume that $g$ is cyclically $Y$-reduced. Therefore one has to obtain equality $\mathbf{0}(g)=v \mathbf{0}(u) v^{-1}$ for the maximal 0-subgroups $\mathbf{0}(g)$ and $\mathbf{0}(u)$ normalized by $g$ and $u$, respectively. By the symmetry, it suffices to prove that $v \mathbf{0}(u) v^{-1}$ is a 0 -subgroup, because it is normalized by $g=v u v^{-1}$.

Let $G, U, V$ be shortest words representing elements $g, u, v$. Then there is an annular diagram diagram over $\mathbf{G}(0)$ whose boundary labels are $G \equiv \phi\left(q_{1}\right)$ and $U \equiv \phi\left(q_{2}^{-1}\right)$ and a path $t$, connecting the initial vertices of $q_{1}$ and $q_{2}$ is labeled by $V$. Arbitrary $Y$-band starting with $q_{1}$ ends on $q_{2}$, and vise versa, since $g$ and $u$ are cyclically $Y$-reduced. Since these words are essential, $\left(q_{1}\right)_{-}$can be connected with a vertex of $q_{2}$ by a path $p$ with label $P$ where $|P|=0$. It defines a decomposition $U \equiv U_{1} U_{2}$. Hence $V=P U_{1}^{-1} U^{k}$ in $\mathbf{G}(0)$ for some integer $k$ by Lemma 11.4 from [30].

Now, $U^{k} \mathbf{0}(U) U^{-k}=\mathbf{0}(U)$, by the definition of $\mathbf{0}(U)=\mathbf{0}(u)$. Then $U_{1}^{-1} \mathbf{0}(U) U_{1}$ is also a 0 -subgroup by Lemma 3.6 since the word $U \equiv U_{1} U_{2}$ normalizes $\mathbf{0}(U)$. Finally, $V \mathbf{0}(U) V^{-1}=$ $P\left(U_{1}^{-1} \mathbf{0}(U) U_{1}\right) P^{-1}$ is a 0 -subgroup because $P$ is a 0 -word. 
The second statement of the lemma immediately follows from the first one.

Lemma 3.8. Suppose that $\mathcal{R}$ satisfies (Z1.1). If $g$ is an essential cyclically $Y$-reduced element of $\mathbf{G}(0)$ and $x \in \mathbf{0}(g)$ then $g^{s} x$ is an essential and cyclically $Y$-reduced element for $s \neq 0$.

Proof. Suppose that $g^{s} x=h^{-1} u h$ for 0 -element $u$ and $h \in \mathbf{G}(0)$. Then the $Y$-length of $\left(g^{s} x\right)^{m}$ is at most $2|h|_{Y}$ for every integer $m \geq 0$. On the other, we have $\left(g^{s} x\right)^{m}=g^{s m} x_{1}$ for some 0-element $x_{1} \in \mathbf{0}(g)$ since the 0 -subgroup $\mathbf{0}(g)$ is normalized by $g$. Therefore $g^{s m}=h^{-1} u^{m} h x_{1}^{-1}$ and the $Y$-length of $g^{s m}$ is at most $2|h|_{Y}$ for every integer $m$. This contradicts the fact that $g^{s m}$ is cyclically $Y$-reduced by the second part of Lemma 3.4.

Similarly if $g^{s} x$ is not cyclically $Y$-reduced then $\left|\left(g^{s} x\right)^{2}\right|_{Y}<2\left|g^{s} x\right|_{Y}=2\left|g^{s}\right|_{Y}$. Therefore for some 0-element $x_{1}$ we have $\left|g^{2 s}\right|_{Y}=\left|g^{2 s} x_{1}\right|_{Y}=\left|\left(g^{s} x\right)^{2}\right|<2\left|g^{s}\right|_{Y}$ which contradicts Lemma 3.4 $(2)$.

Lemma 3.9. Suppose that $\mathcal{R}$ satisfies (Z1.1). If $g$ is an essential element and $x \in \mathbf{0}(g)$ then $\mathbf{0}(g x)=\mathbf{0}(g)$.

Proof. By Lemma 3.7 we can assume that $g$ is cyclically $Y$-reduced. Since $x \in \mathbf{0}(g)$, the subgroup $\mathbf{0}(g)$ which is normalized by $g$, is also normalized by $g x$. Since by Lemma $3.8, g x$ is also cyclically $Y$-reduced, we have $\mathbf{0}(g) \subseteq \mathbf{0}(g x)$. In particular $x \in \mathbf{0}(g x)$, so $x^{-1} \in \mathbf{0}(g x)$. Therefore, as above, $\mathbf{0}(g x) \subseteq \mathbf{0}\left(g x x^{-1}\right)=\mathbf{0}(g)$. Hence $\mathbf{0}(g)=\mathbf{0}(g x)$.

Lemma 3.10. Suppose that $\mathcal{R}$ satisfies (Z1.1). If $g$ is an essential element then for every integer $m \neq 0$, element $g^{m}$ is essential and $\mathbf{0}\left(g^{m}\right)=\mathbf{0}(g)$

Proof. By Lemma 3.7 we can assume that $g$ is cyclically $Y$-reduced. By Lemma 3.4 (2) one may assume that $g^{m}$ is also cyclically $Y$-reduced. Then $g^{m}$ is essential by lemmas $3.4(2)$ and 3.3 , and subgroups $\mathbf{0}(g)$ and $\mathbf{0}\left(g^{m}\right)$ are 0 -subgroups. It is clear that $\mathbf{0}(g) \subseteq \mathbf{0}\left(g^{m}\right)$. Let $x \in \mathbf{0}\left(g^{m}\right)$. Then $x \in \mathbf{0}\left(g^{m s}\right)$ for any $s \neq 0$. Take any integer $t \neq 0$. We need to show that $g^{-t} x g^{t}$ is a 0 -word. But we may choose $s$ so that $g^{m s}=g^{t} g^{t^{\prime}}$ for some $t^{\prime}$ and $\left|g^{m s}\right|=\left|g^{t}\right|+\left|g^{t^{\prime}}\right|$ by Lemma 3.3. Then $g^{-t} x g^{t}$ is a 0 -word by Lemma 3.6.

Lemma 3.11. Suppose that $\mathcal{R}$ satisfies (Z1.1). If $g$ is an essential element of $\mathbf{G}(0)$ and $x \in \mathbf{0}(g)$, $l \neq 0$, then $g^{l} x$ is an essential element and $\mathbf{0}\left(g^{l} x\right)=\mathbf{0}(g)$.

Proof. The claim follows immediately from lemmas 3.10, 3.9, 3.8 and 3.7.

Lemma 3.12. Suppose that $\mathcal{R}$ satisfies conditions (Z1.1), (Z1.2) and (Z3.2). Then for every essential element $g \in \mathbf{G}(0)$ which is cyclically minimal in rank $1 / 2$, and every $x \in \mathbf{0}(g)$, we have $(g x)^{n}=g^{n}$.

Proof. By the definition of $\mathbf{0}(g)$ and Lemma 3.7, we can assume that $g$ is cyclically reduced and $x$ is a 0 -element. We have $(g x)^{n}=g^{n}\left(g^{-n+1} x g^{n-1}\right) \ldots\left(g^{-1} x g\right) x$. By by property (Z3.2), there exists a 0 -element $r$ such that $g r^{-1}$ commutes with every element of $\mathbf{0}(g)$. Therefore $r$ also normalizes $\mathbf{0}(g)$ and the actions of $r$ and $g$ on $\mathbf{0}(g)$ (by conjugation) coincide. Hence for every integer $s$ the actions of $r^{s}$ and $g^{s}$ on $\mathbf{0}(g)$ also coincide, so $g^{-s} x g^{s}=r^{-s} x r^{s}$. Therefore

$$
\left(g^{-n+1} x g^{n-1}\right) \ldots\left(g^{-1} x g\right) x=\left(r^{-n+1} x r^{n-1}\right) \ldots\left(r^{-1} x r\right) x=r^{-n}(r x)^{n} .
$$

By property $(\mathrm{Z} 1.2)(r x)^{n}=r^{n}=1$ in $\mathbf{G}(0)$. Hence $(g x)^{n}=g^{n}$. 
Lemma 3.13. Suppose that $\mathcal{R}$ satisfies conditions (Z1.1), (Z1.2), (Z3.2). Then for every essential element $g \in \mathbf{G}(0)$ which is cyclically minimal in rank $1 / 2$, and every $x \in \mathbf{0}(g)$ we have $g^{n} x=x g^{n}$.

Proof. It follows from Lemma 3.12 that $g^{n}$ commutes with both $g$ and $g x$. Therefore it commutes with $x$.

Lemma 3.14. Suppose that $\mathcal{R}$ satisfies (Z1.1). Let $A$ be a $Y$-reduced essential word over $\mathcal{X}$ (i.e. it represents an essential element in $\mathbf{G}(0)$ ) and let $C$ be a 0 -word. Suppose that the subgroup generated by $A^{-s} C A^{s}$ for all integers $s$ consists of 0-elements. Then $C \in \mathbf{0}(A)$.

Proof. If $A$ is cyclically $Y$-reduced then $C \in \mathbf{0}(A)$ by definition. Otherwise by Lemma 3.3 (2), $A$ can be represented as $U V U^{\prime}$ where $U^{\prime} U$ is equal to a 0 -word $W$ in $\mathbf{G}(0)$ and $V$ is cyclically $Y$-reduced, so every cyclic shift of $V$ is $Y$-reduced. Since we assume that $A$ is essential, $V W$ is an essential word. By Lemma 3.3 (1) then every cyclic shift of $V W$ is $Y$-reduced. Hence $V W$ is cyclically $Y$-reduced. By Lemma 3.6, $U^{-1} C U$ is a 0 -element. Since the subgroup generated by $A^{-s} C A^{s}, s \in \mathbb{Z}$ consists of 0 -elements and is normalized by $\left.A, U(V W)^{-s}\left(U^{-1} C U\right)(V W)^{s}\right) U^{-1}$ is a 0 -element for every $s$.

Notice that $U(V W)^{s} U^{-1}$ is a $Y$-reduced form of $A^{s}$. Indeed, $V$ is cyclically $Y$-reduced subword of $A$ by definition, $V W$ is a conjugate of $A$ of the same $Y$-length as $V$. Hence by Lemma $3.3(2)$, $V W$ is cyclically $Y$-reduced. By Lemma $3.4(2)$ then $(V W)^{s}$ is cyclically $Y$-reduced. By Lemma 3.3(1), $U(V W)^{s} U^{-1}$ is reduced because every subword of $Y$-length 2 of $U(V W)^{s} U^{-1}$ is either a subword of $A$ or a subword of $(V W)^{s}$.

Since $\left.A^{-s} C A^{s}=U(V W)^{-s}\left(U^{-1} C U\right)(V W)^{s}\right) U^{-1}$ is a 0 -element for every $s$, and $U^{\prime} \equiv W U^{-1}$ is a suffix of $A^{s}$, by Lemma 3.6, the subgroup generated by $(V W)^{-s}\left(U^{-1} C U\right)(V W)^{s}$ consists of 0 elements and is normalized by $U^{-1} A U=V W$. Hence $U^{-1} C U$ is in $\mathbf{0}\left(U^{-1} A U\right)$. Therefore $C \in \mathbf{0}(A)$ by Lemma 3.7.

Lemma 3.15. Suppose that $\mathcal{R}$ satisfies (Z1.1), (Z1.2), (Z3.1) and (Z3.2). Let $g$ be an essential element of $\mathbf{G}(0)$ represented by a cyclically $Y$-reduced word $A$ in $\mathbf{G}(0)$. Suppose that $A$ contains a subword $B^{6}$, where $B$ is a cyclically minimal in rank $1 / 2$ word of a positive $Y$-length. Let $A^{\prime}$ be the word obtained by replacing that occurrence of $B^{6}$ by $B^{6-n}$. Then $\mathbf{0}(A) \leq \mathbf{0}\left(A^{\prime}\right)$, provided the word $A^{\prime}$ represents an essential element of $\mathbf{G}(0)$.

Proof. Let $x \in \mathbf{0}(A)$. Then for every integer $s, g^{-s} x g^{s}$ is a 0 -element. Fix $s>0$. Let $C$ be a 0 -word representing $x, D$ be the word representing $g^{-s} x g^{s}$. Notice that $B^{6}$ contains the fifth power of every cyclic shift of $B$. Let $B_{1}$ be a cyclic shift of $B$ starting with a letter from $Y$. Let $A \equiv A_{1} B_{1}^{5} A_{2}$. Clearly then the word $A^{\prime}$ obtained from $A$ by replacing $B^{6}$ by $B^{6-n}$ is equal to $A_{1} B_{1}^{5-n} A_{2}$ in the free group.

Consider a van Kampen diagram over $\mathbf{G}(0)$ with boundary label $p_{1} q_{1} p_{2}^{-1} q_{2}^{-1}$ where $\phi\left(p_{1}\right)=C$, $\phi\left(p_{2}\right)=D, \phi\left(q_{1}\right)=\phi\left(q_{2}\right)=A^{s}$. The path $q_{1}$ can be represented as

$$
q_{1}=t_{1,1} t_{1,2} t_{1,3} t_{1,4} t_{2,1} t_{2,2} t_{2,3} t_{2,4} \ldots t_{s, 1} t_{s, 2} t_{s, 3} t_{s, 4}
$$

where $\phi\left(t_{i, 2}\right) \equiv B_{1}^{4}, \phi\left(t_{i, 3}\right) \equiv B_{1}, i=1, \ldots, s\left(\phi\left(t_{i, 2} t_{i, 3}\right)\right.$ is the fixed occurrence of $B_{1}^{5}$ in $\left.A\right)$.

Since $A^{s}$ is $Y$-reduced and $p_{1}, p_{2}$ do not contain $Y$-edges, the maximal $Y$-bands starting on $q_{1}$ provide a one-to-one correspondence between $Y$-edges of $q_{1}$ and $Y$-edges of $q_{2}$. Since $B_{1}$ starts with a $Y$-letter, $t_{i, 2}$ and $t_{i, 3}$ start with a $Y$-edges, $e_{i}$ and $f_{i}$ respectively, $i=1, \ldots, s$. 
For every $i=1, \ldots, s$, consider the maximal $Y$-band $\mathcal{T}_{i}$ starting on $e_{i}$ and the maximal $Y$-band $\mathcal{B}_{i}$ starting on $f_{i}$. Consider the smallest subdiagram $\Delta_{i}$ bounded by a side of $\mathcal{T}_{i}$, a side of $\mathcal{B}_{i}, q_{1}$, $q_{2}$, containing $\mathcal{T}_{i}$ but not containing $\mathcal{B}_{i}$. The boundary of each $\Delta_{i}$ has the form $p_{i}^{\prime} t_{i, 2}\left(p_{i}^{\prime \prime}\right)^{-1}\left(t_{i}^{\prime}\right)^{-1}$ where $\phi\left(t_{i, 2}\right) \equiv \phi\left(t_{i}^{\prime}\right) \equiv B_{1}^{4}, v_{i} \equiv \phi\left(p_{i}^{\prime}\right)$ and $v_{i}^{\prime} \equiv \phi\left(p_{i}^{\prime \prime}\right)$ are 0 -words. Therefore

$$
B_{1}^{-4} v_{i} B_{1}^{4}=v_{i}^{\prime}
$$

for every $i=1, \ldots, s$.

By Property (Z3.1) (all premises of (Z3.1) hold), $v_{i} \in \mathbf{0}\left(B_{1}\right)$. By Lemma 3.13, $v_{i}$ commutes with $B_{1}^{n}$. For every $i=1, \ldots, s$ consider a van Kampen diagram $\Gamma_{i}$ over $\mathbf{G}(0)$ with boundary $\bar{p}_{i} \bar{q}_{i}\left(\bar{p}_{i}^{\prime}\right)^{-1}\left(\bar{q}_{i}^{\prime}\right)^{-1}$ where $\phi\left(\bar{p}_{i}\right) \equiv \phi\left(\bar{p}_{i}^{\prime}\right) \equiv v_{i}, \phi\left(\bar{q}_{i}\right) \equiv \phi\left(\bar{q}_{i}^{\prime}\right) \equiv B_{1}^{-n}$. Now cut the diagram $\Delta$ along each path $p_{i}$. Thus each path $p_{i}^{\prime}$ turns into two copies of $p_{i}^{\prime}$, which we shall call the left copy and the right copy: the right copy is contained in the diagram containing the band $\mathcal{T}_{i}$. For every $i=1, \ldots, s$, identify paths $\bar{p}_{i}$ of $\Gamma_{i}$ with the left copy of $p_{i}, \bar{p}_{i}^{\prime}$ with the right copy of $p_{i}, i=1, \ldots, s$. As a result, we get a van Kampen diagram with boundary label $\left(A_{1} B_{1}^{-n} B_{1}^{5} A_{2}\right)^{-s} C\left(A_{1} B_{1}^{-n} B_{1}^{5} A_{2}\right)^{s} D^{-1}$. Since as we have noticed, $A_{1} B_{1}^{5-n} A_{2}=A^{\prime}$, we have that $\left(A^{\prime}\right)^{-s} C\left(A^{\prime}\right)^{s}=D$ in $\mathbf{G}(0)$ for every positive $s$. The same result for negative $s$ can be proved similarly.

Hence the subgroup generated by $\left(A^{\prime}\right)^{-s} C\left(A^{\prime}\right)^{s}, s \in \mathbb{Z}$, consists of 0 -elements and is normalized by $A^{\prime}$. By Lemma 3.14, $C \in \mathbf{0}\left(A^{\prime}\right)$ as required.

Lemma 3.16. Suppose that $\mathcal{R}$ satisfies (Z1.1), (Z2.1), (Z2.2), (Z2.3). Let $g$ be an essential element of $\mathbf{G}(0)$ represented by a cyclically $Y$-reduced word $A$ in $\mathbf{G}(0)$. Suppose that $A$ contains a subword $B$ which is a prefix of a cyclic shift $C$ of a hub, $C \equiv B B^{\prime}$, and $|B| \geq \varepsilon|C|$. Let $A^{\prime}$ be the word obtained by replacing that occurrence of $B$ by $\left(B^{\prime}\right)^{-1}$. Then, provided $A^{\prime}$ represents an essential element of $\mathbf{G}(0)$, we have inclusion $\mathbf{0}(A) \leq \mathbf{0}\left(A^{\prime}\right)$.

Proof. The proof is similar to the proof of Lemma 3.15. Let $x \in \mathbf{0}(A)$. Then for every integer $s$, $g^{-s} x g^{s}$ is a 0 -element. Fix $s>0$. Let $C$ be a 0 -word representing $x, D$ be the word representing $g^{-s} x g^{s}$. Since letters not from $Y$ have $Y$-length 0, we can assume that $B$ starts and ends with $Y$-letters. Let $A \equiv A_{1} B A_{2}$. Clearly then the word $A^{\prime}$ obtained from $A$ by replacing $B$ by $\left(B^{\prime}\right)^{-1}$ is equal to $A_{1}\left(B^{\prime}\right)^{-1} A_{2}$ in the free group.

Consider a van Kampen diagram with boundary label $p_{1} q_{1} p_{2}^{-1} q_{2}^{-1}$ where $\phi\left(p_{1}\right) \equiv C, \phi\left(p_{2}\right) \equiv D$, $\phi\left(q_{1}\right) \equiv \phi\left(q_{2}\right) \equiv A^{s}$. The path $q_{1}$ can be represented as

$$
q_{1}=t_{1,1} t_{1,2} t_{1,3} t_{2,1} t_{2,2} t_{2,3} \ldots t_{s, 1} t_{s, 2} t_{s, 3}
$$

where $\phi\left(t_{i, 2}\right) \equiv B, i=1, \ldots, s\left(\phi\left(t_{i, 2}\right)\right.$ is the fixed occurrence of $B$ in $\left.A\right)$.

Since $A^{s}$ is $Y$-reduced by Lemma $3.4(2)$, and $p_{1}, p_{2}$ do not contain $Y$-edges, the maximal $Y$ bands starting on $q_{1}$ provide a one-to-one correspondence between $Y$-edges of $q_{1}$ and $Y$-edges of $q_{2}$. Since $B$ starts and ends with a $Y$-letter, $t_{i, 2}$ starts and ends with a $Y$-edges, $e_{i}$ and $f_{i}$ respectively, $i=1, \ldots, s$.

For every $i=1, \ldots, s$, consider the maximal $Y$-band $\mathcal{T}_{i}$ starting on $e_{i}$ and the maximal $Y$-band $\mathcal{B}_{i}$ starting on $f_{i}$. Consider the smallest subdiagram $\Delta_{i}$ bounded by a side of $\mathcal{T}_{i}$, a side of $\mathcal{B}_{i}$, $q_{1}$, and $q_{2}$, containing $\mathcal{T}_{i}$ and $\mathcal{B}_{i}$. The boundary of each $\Delta_{i}$ has the form $p_{i}^{\prime} t_{i, 2}\left(p_{i}^{\prime \prime}\right)^{-1}\left(t_{i}^{\prime}\right)^{-1}$ where $\phi\left(t_{i, 2}\right) \equiv \phi\left(t_{i}^{\prime}\right) \equiv B, v_{i} \equiv \phi\left(p_{i}^{\prime}\right)$ and $v_{i}^{\prime} \equiv \phi\left(p_{i}^{\prime \prime}\right)$ are 0 -words. Therefore

$$
v_{i} B=B v_{i}^{\prime}
$$


for every $i=1, \ldots, s$.

By Property (Z2.3) (all premises of (Z2.3) hold), we have

$$
v_{i}\left(B^{\prime}\right)^{-1}=\left(B^{\prime}\right)^{-1} v_{i}^{\prime}, i=1, \ldots, s .
$$

Thus for every $i=1, \ldots, s$ there exists a van Kampen diagram $\Gamma_{i}$ over $\mathcal{R}$ with boundary $p_{i}^{\prime} \bar{t}_{i, 2}\left(p_{i}^{\prime \prime}\right)^{-1}\left(\bar{t}_{i}^{\prime}\right)^{-1}$ where $\phi\left(\bar{t}_{i, 2}\right) \equiv\left(B^{\prime}\right)^{-1}, \phi\left(\bar{t}_{i}^{\prime}\right) \equiv\left(B^{\prime}\right)^{-1}$. Now we can substitute the subdiagrams $\Delta_{i}$ in $\Delta$ by $\Gamma_{i}$. The boundary label of the resulting diagram is equal $\left(A^{\prime}\right)^{-s} C\left(A^{\prime}\right)^{s} D^{-1}$. Hence $\left(A^{\prime}\right)^{-s} C\left(A^{\prime}\right)^{s}=D$ in $\mathbf{G}(0)$ for every positive $s$. The same result for negative $s$ can be proved similarly.

Hence the subgroup generated by $\left(A^{\prime}\right)^{-s} C\left(A^{\prime}\right)^{s}, s \in \mathbb{Z}$, consists of 0-elements and is normalized by $A^{\prime}$. By Lemma $3.14 C \in \mathbf{0}\left(A^{\prime}\right)$ as required.

\subsection{The construction of a graded presentation}

In this section, we show how the theory from [30, Chapter 6] can be adapted to diagrams over a presentation satisfying (Z1), (Z2), (Z3).

We are defining several concepts analogous to concepts in [30], chapter 6, by induction on the rank $i$.

We say that a certain statement holds in rank $i$ if it holds in the group $\mathbf{G}(i)$ given by $\mathcal{R}_{i}=$ $\cup_{j=0}^{i} \mathcal{S}_{j}$.

Recall that a word $w$ is A-periodic if it is a subword of some power of the word $A$. Any $A$ periodic word can be read (say, clockwise) on a cyclic graph with $|A|$ edges, labeled, starting with a vertex $o$, by the word $A$. Then every $A$-periodic word corresponds to a path on this graph. Let $W \equiv W_{1} W_{2}$ be a decomposition of an $A$-periodic word $W$, such that the word $W_{1}$ ends at $o$, when reading the word $W$ on the cyclic graph, $|W| \geq|A|$. Then we say that the decomposition is a phase decomposition of $W$. If the label of a simple path $p$ of an arbitrary labeled graph has an $A$-periodic label $W \equiv \phi(p)$, then a vertex of $p$, defining a phase decomposition of $p$ is said to be a phase vertex of $p$.

Up to the end of this section, the $Y$-length of a word $W$ will be called simply length and will be denoted by $|W|$.

Let us define simple words of rank $i=0,1 / 2,1,2, \ldots$ and periods of rank $i=1,2,3, \ldots$ Let $\mathcal{S}_{0}=\mathcal{R}_{0}$ and $\mathcal{S}_{1 / 2}$ be the set of hubs. Thus the groups $\mathbf{G}(0)$ and $\mathbf{G}(1 / 2)=\mathbf{G}$ are defined by relations of the sets $\mathcal{R}_{0}$ and $\mathcal{R}_{1 / 2}=\mathcal{S}_{0} \cup \mathcal{S}_{1 / 2}$, respectively. A word $A$ of positive length is said to be simple in rank 0 (resp. 1/2) if it is not a conjugate in rank 0 (resp. 1/2) either of a word of length 0 or of a product of the form $B^{l} P$, where $|B|<|A|$ and $P \in \mathbf{0}(B)$ in rank 0 .

Suppose that $i \geq 1$, and we have defined the sets of relators $\mathcal{R}_{j}, j<i$, and the corresponding groups $\mathbf{G}(j)$ of ranks $j<i$. Suppose also that we have defined simple words of ranks $j, j<i$, and periods of rank $j, 1 \leq j<i$.

For every $i=1 / 2,1,2,3, .$. let $i_{-}$denote $i-1$ if $i>1,1 / 2$ if $i=1$ or 0 if $i=1 / 2$. Similarly $i_{+}$ is $1 / 2$ if $i=0$ or 1 if $i=1 / 2$ or $i+1$ if $i \geq 1$.

For every $i \geq 1$ consider the set $\mathcal{X}_{i}^{\prime}$ of all words of length $i$ which are simple in rank $i_{-}$, and the equivalence $\sim_{i_{-}}$given in Lemma 3.24 (we apply it for the smaller rank here). Now choose a set of representatives $\mathcal{X}_{i}$ of the equivalence classes of $\mathcal{X}_{i}^{\prime}$. The words of $\mathcal{X}_{i}$ are said to be periods of rank $i$. The set of words $\mathcal{R}_{i}$ (defining the group $\mathbf{G}(i)$ or rank $i$ ) is the union of $\mathcal{R}_{i_{-}}$and $\mathcal{S}_{i}=\left\{A^{n}, A \in \mathcal{X}_{i}\right.$. 
Let $A$ be a word of positive length. We say that $A$ is simple in rank $i \geq 1$ if it is not a conjugate in rank $i$ either of a word of length 0 or of a word of the form $B^{l} P$, where $B$ is a period of rank $j \leq i$ or an essential word with $|B|<|A|$, and $P$ represents a word of the subgroup $\mathbf{0}(B)$.

Let $\mathcal{R}(\infty)=\cup_{0}^{\infty} \mathcal{R}_{i}$. If $\Pi$ is a cell of a diagram over $\mathcal{R}(\infty)$ with the boundary labeled by a word of the set $\mathcal{S}_{j}$, then, by definition, $r(\Pi)=j$. The boundary label of every cell of a rank $\geq 1$ (read counterclockwise), has a period $A$ defined up to cyclic shifts.

For $j \geq 1$, a pair of distinct cells $\Pi_{1}$ and $\Pi_{2}$ of rank $j$ of a diagram $\Delta$ is said to be a $j$-pair, if their counterclockwise contours $p_{1}$ and $p_{2}$ are labeled by $A^{n}$ and $A^{-n}$ for a period $A$ of rank $j$ and there is a path $t=\left(p_{1}\right)_{-}-\left(p_{2}\right)_{-}$without self-intersections such that $\phi(t)$ is equal, in rank $j_{-}$, to an element of $\mathbf{0}(A)$. Then $\phi(t)$ and $A^{n}$ commute in rank $j_{-}$by Lemma 3.13, and so the subdiagram with contour $p_{1} t p_{2} t^{-1}$ can be replaced in $\Delta$ by a diagram of rank $j_{-}$. As a result, we obtain a diagram with the same boundary label as $\Delta$ but of a smaller type.

Similarly, a pair of hubs $\Pi_{1}, \Pi_{2}$ with counterclockwise contours $p_{1}, p_{2}$ forms a $\frac{1}{2}$-pair, if the vertices $\left(p_{1}\right)_{-}$and $\left(p_{2}\right)_{\text {- }}$ are connected by a path $t$ without self-intersections such that the label of the path $p_{1} t p_{2} t^{-1}$ is equal to 1 in $\mathbf{G}(0)$. Consequently, any diagram can be replaced by a diagram having no $j$-pairs, $j=1 / 2,1,2, \ldots$, i.e. by a $g$-reduced diagram with the same boundary label(s $)^{8}$.

Assume that $q_{1}$ and $q_{2}$ are disjoint sections of the boundary $\partial(\Delta)$ of a diagram $\Delta$ of rank $i$, and $\phi\left(q_{1}\right), \phi\left(q_{2}\right)$ are $A$ - and $A^{-1}$-periodic words respectively, where $\left|q_{1}\right|,\left|q_{2}\right| \geq|A|, A$ is simple in rank $i$ or a period of rank $j \leq i$. We call $q_{1}$ and $q_{2}$ compatible if there exist phase vertices $o_{1}$ and $o_{2}$ on $q_{1}$ and $q_{2}$, respectively, and a path $t=o_{1}-o_{2}$ without self-intersections, such that $\phi(t)$ is equal in rank $i$ to an element of $\mathbf{0}(A)$; moreover the equality must be true in rank $j_{-}$if $A$ is a period of rank $j$.

Similarly one defines the compatibility of an $A$-periodic cell $\Pi$ of rank $j \geq 1$ with a $A^{ \pm 1}$-periodic section $q$ of $\partial(\Delta)$.

Lemma 3.17. Let $\Pi_{1}$ be a cell of rank $j \geq 1$ in a g-reduced diagram $\Delta$ of rank $i$. Assume it has a boundary label $A^{n}$. Let $\Gamma$ be a subdiagram of $\Delta$ with contour $p_{1} q_{1} p_{2} q_{2}$, where $q_{1}$ is a subpath of $\partial\left(\Pi_{1}\right)$ and $q_{2}$ is a subpath of the contour of a cell $\Pi_{2}$ with the boundary label $A^{-n},\left(\Pi_{1}, \Pi_{2}\right.$ do not belong to $\Gamma)$. Then

1) $q_{1}$ and $q_{2}$ cannot be compatible in $\Gamma$;

2) the subdiagram $\Gamma$ contains no cells compatible with $q_{1}$.

Proof. If one of the statements were false, we could include the cell $\Pi_{1}$ into a $j$-pair and come to an obvious contradiction with the assumption that $\Delta$ is g-reduced diagram, as in Lemma 13.2 from [30].

Lemma 3.18. Let $p_{1}, p_{2} \ldots$ be a partition of the boundary of a diagram $\Delta$ of rank $i$ into several sections, and $\phi\left(p_{1}\right) \equiv A^{l}$ for some period of rank $j$. Assume that a cell $\Pi$ of $\Delta$ is compatible with $p_{1}$. Then there exists a g-reduced diagram $\Delta^{\prime}$ with topologically identical partition of the boundary into $p_{1}^{\prime}, p_{2}^{\prime}, \ldots$, such that $\tau\left(\Delta^{\prime}\right)<\tau(\Delta), \phi\left(p_{k}^{\prime}\right) \equiv \phi\left(p_{k}\right)$ for $k \geq 2, \phi\left(p_{1}^{\prime}\right) \equiv A^{l+s n}$ for some integer $s$, and $\Delta^{\prime}$ has no cells compatible with $p_{1}$.

\footnotetext{
${ }^{8}$ Recall that in [30], $\$ 13.2$, such diagrams are called reduced. But in this paper we shall consider two kinds of reduced diagrams, so we call diagrams without $j$-pairs g-reduced; "g" stands for "graded".
} 
Proof. Let $t$ be the path connecting a phase vertex $o_{1}$ of $p_{1}$ and a phase vertex $o_{2}$ of the $A^{ \pm 1}$ periodic cell $\Pi$, according to the definition of the compatibility, and $T \equiv \phi(t)$ is equal in rank $i$ to an element of $\mathbf{0}(A)$. The vertex $o_{1}$ defines a decomposition $A^{l_{1}} A^{l_{2}}$, and there is a path $p$ homotopic to $p_{1}$ with label $W \equiv A^{l_{1}} T A^{ \pm n} T^{-1} A^{l_{2}}$. By Lemma 3.13, we have $W=A^{l \pm n}$ in rank $j_{-}$. Thus one can cut $\Pi$ out of $\Delta$, by cutting along $p$. Then one can replace the deleted subdiagram by the diagram of the equality $W=A^{l \pm n}$. By continuing this process, we prove the lemma.

The following proposition is the main statement of this section.

Proposition 3.19. If the presentation $\mathcal{R}$ of a group $\mathbf{G}$ satisfies properties (Z1), (Z2), (Z3) then the graded presentation $\mathcal{S}_{0} \cup \mathcal{S}_{1 / 2} \cup \mathcal{S}_{1} \cup \mathcal{S}_{2} \cup \ldots$ satisfies statements of Lemmas 3.20 through 3.39 below and is such that $\mathcal{R}=\mathcal{S}_{0} \cup \mathcal{S}_{1 / 2}$. In particular, all g-reduced diagrams over this presentation are A-maps. Every relator from $\mathcal{S}_{i}, i \geq 1$, is of the form $A^{n}$ for a cyclically reduced word $A$ of $Y$-length $i$. The group defined by this presentation is $\mathbf{G} /\left\langle g^{n}, g \in \mathbf{G}\right\rangle$.

Lemmas from 3.20 to 3.39 will be proved by simultaneous induction on rank $i \geq \frac{1}{2}$.

Lemma 3.20. Let $V$ be a periodic word with a simple in rank $i$ period. Assume that for some word $U$ with $2|U| \leq|V|$, the word $V U$ is a conjugate in rank $i$ of a 0-word. Then there is a decomposition $V \equiv V_{1} V_{2}$, such that ||$V_{1}|-| V_{2}||<\beta|V|+|U|$ and for some word $S$ of length at most $3 \gamma|V|, V_{2} U V_{1}=S$ in rank $i$.

Proof. Consider a g-reduced annular diagram $\Delta$ for the conjugacy of $V U$ to a 0 -word $W$. We may assume that $\Delta$ has the smallest possible type. Therefore there are no $Y$-bands surrounding the hole of $\Delta$, since otherwise their boundary labels were 0 -words. Hence, by lemmas 3.38 and 2.16, there are vertices $o_{1}$ on the contour $q_{1}$ labeled by $V U$ and $o_{2}$ on the contour $q_{2}$ labeled by $W$, which can be connected by a simple path $t$ of length at most $\gamma(|V|+|U|) \leq 3 \gamma|V| / 2$ (see Figure 7).

One may suppose that the vertex $o_{2}$ does not cut the word $W$, because $|W|=0$. The vertex $o_{1}$ cannot cut the word $|U|$. Indeed, in case it defines a decomposition $U \equiv U_{1} U_{2}$, we obtain the following equality in rank $i$ : $U_{1} T W^{-1} T^{-1} U_{2}=V^{-1}$, where $T \equiv \phi(t)$. It follows by lemmas 3.39 and 2.7 that $\bar{\beta}|V|<|U|+2 \gamma(|V|+|U|)$, whence $|V|<(\bar{\beta}-2 \gamma)^{-1}(1+2 \gamma)|U| \leq 2|U|$, contrary to the hypothesis of the lemma.

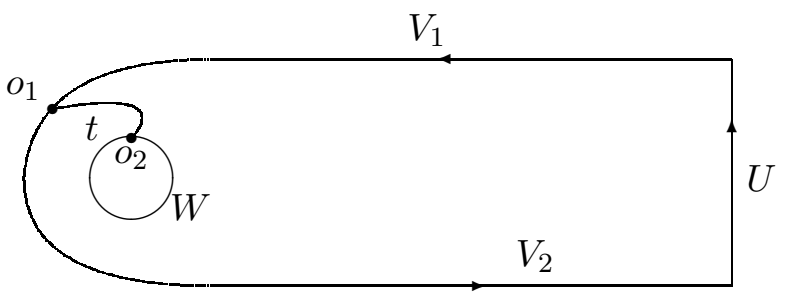

Fig. 7.

Thus the vertex $o_{1}$ defines a decomposition $V \equiv V_{1} V_{2}$, and $T W^{-1} T^{-1} V_{2} U=V_{1}^{-1}$ in rank $i$. Then, as above, $\bar{\beta}\left|V_{1}\right|<\left|V_{2}\right|+|U|+2 \gamma(|V|+|U|)$. Therefore $\left|V_{1}\right|-\left|V_{2}\right|<\beta\left|V_{1}\right|+|U|+3 \gamma|V|$. Similarly, $\left|V_{2}\right|-\left|V_{1}\right|<\beta\left|V_{1}\right|+|U|+3 \gamma|V|$, and so ||$V_{1}|-| V_{2}||<\beta|V|+|U|$, as $2|U| \leq|V|$.

To complete the proof we observe that the product $V_{2} U V_{1}$ is equal in rank $i$ to the word $S \equiv T W T^{-1}$ of length at most $3 \gamma|V|$. 
Let $q_{1}, q_{2}$ be two paths labeled by $A^{ \pm 1}$-periodic words for some period $A$. We say that vertices $o_{1}, o_{2}$ of these paths are in the same phase (with respect to $A$ ) if they are phase vertices for the paths $q_{1}, q_{2}$ with respect to a cyclic permutation $A^{\prime}$ of the word $A$.

Lemma 3.21. Let $\Pi$ be a cell of positive rank in a g-reduced disc diagram $\Delta$ of rank $i$ with contour $p_{1} q_{1} p_{2} q_{2}$, where $q_{1}$ and $q_{2}^{-1}$ are labeled by $A$-periodic words where $A$ is a simple in rank $i$ word. Suppose there are disjoint contiguity subdiagrams $\Gamma_{j}$ of $\Pi$ to $q_{j}$ of degrees $\psi_{j}$ for $j=1,2$, with $\psi_{1}+\psi_{2}>1-\alpha / 2$. Denote $s_{1}^{j} t_{1}^{j} s_{2}^{j} t_{2}^{j}=\partial\left(\Pi, \Gamma_{j}, q_{j}\right)$ (see Figure 8). Let $p$ be a subpath of $q_{2}^{-1}$, such that vertex $o=\left(s_{2}^{2}\right)_{+}$belongs to $p$, where the lengths of subpaths $p^{\prime}=p_{-}-o$ and $p^{\prime \prime}=o-p_{+}$are less than $\frac{1}{3}|\partial(\Pi)|$. Then any vertex $o_{2}$ of $p$ is not in the same phase with vertex $o_{1}=\left(s_{1}^{1}\right)_{-}$.

Proof. By lemmas 3.38, 3.39 and 2.3,

$$
\psi_{1}, \psi_{2} \in[1 / 2-2 \alpha, \bar{\alpha}]
$$

and by Lemma 2.2,

$$
\left|t_{2}^{1}\right|,\left|t_{2}^{2}\right| \in\left[(1+2 \beta)^{-1}(1 / 2-2 \alpha)|\partial(\Pi)|,(1-2 \beta)^{-1} \bar{\alpha}|\partial(\Pi)|\right]
$$

Assume that $o_{2}$ and $o_{1}$ are in the same phase and $o_{2} \in p^{\prime}$. First, let $\Pi$ be a hub. Then $r\left(\Gamma_{1}\right)=r\left(\Gamma_{2}\right)=0$ by Lemma 3.37 in the smaller rank, hence $\left|s_{l}^{j}\right|=0, l, j=1,2$. Recall that by (Z2.2), every hub is a linear word. By definition, every simple word in $\operatorname{rank} i$ is cyclically $Y$-reduced.

Therefore by Lemmas 3.2 and 3.4 every maximal $Y$-band starting on $t_{l}^{j}$ ends on $t_{3-l}^{j}$ and forms a 0-bond between these paths. Hence any subpath of $t_{l}^{j}$ starting and ending with $Y$-letters, is a contiguity arc for some contiguity subdiagram between $t_{l}^{j}$ and $t_{3-l}^{j}$ for $l, j=1,2$. By our assumption and inequality (11), there are subpaths $u^{1}$ and $u^{2}$ of $t_{2}^{1}$ and $\left(t_{2}^{2}\right)^{-1}$ with the same label $U$, such that

$$
|U|>\left((1+2 \beta)^{-1}(1 / 2-2 \alpha)-1 / 3\right)|\partial(\Pi)|>\frac{1}{7}|\partial(\Pi)|
$$

Gluing along $u_{1}$ and $u_{2}$ one can construct a contiguity diagram of rank 0 between disjoint arcs of the cell $\Pi$ of length greater than $\frac{1}{7}|\partial(\Pi)|$. But this contradicts condition (Z2.2).

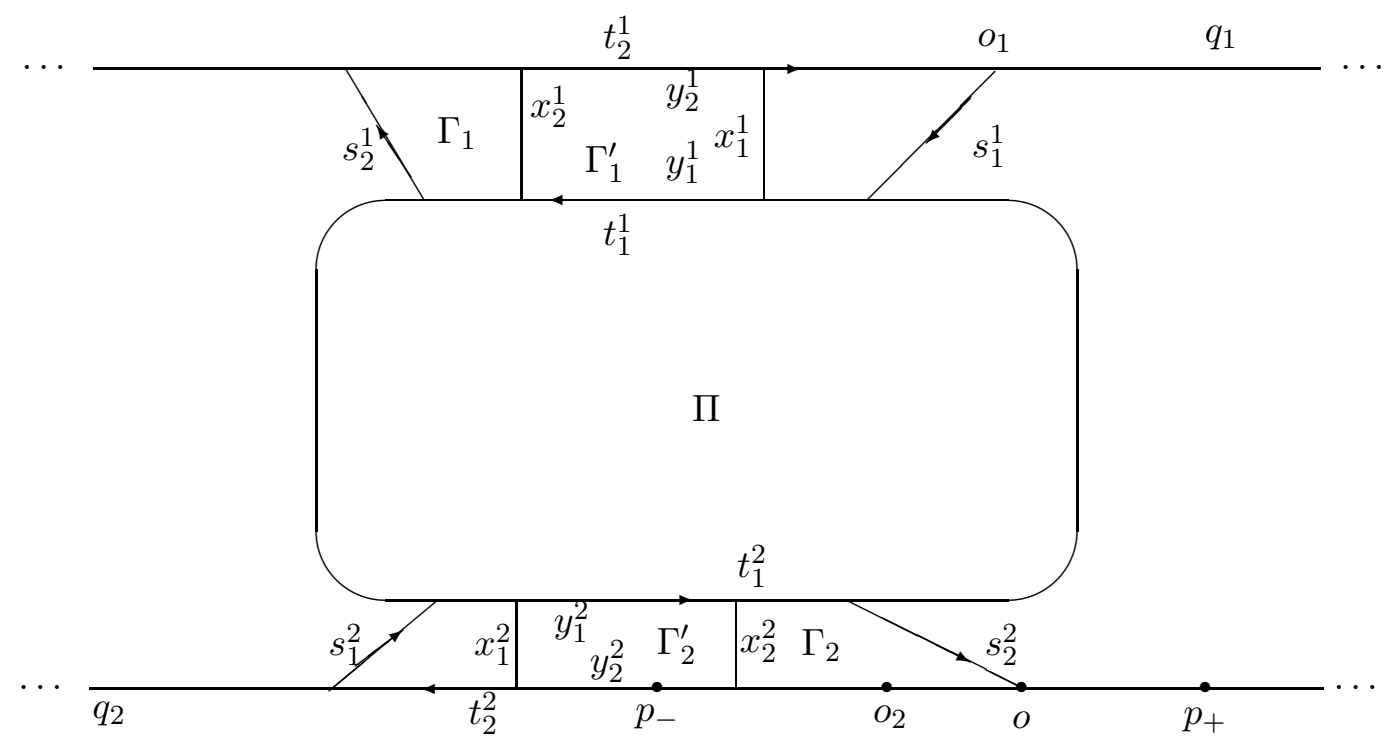


Fig. 8.

Now assume that $\Pi$ is a cell with a period $B$ of rank $k \leq i$. In this case, $r\left(\Gamma_{1}\right), r\left(\Gamma_{2}\right)<k$ by lemmas 2.1, 2.14 and 3.39. As in the previous paragraph, there are subpaths $u_{1}$ and $u_{2}$ of $t_{2}^{1}$ and $\left(t_{2}^{2}\right)^{-1}$ with the same label $U$, where $|U|>\frac{1}{7}|\partial(\Pi)|$.

Lemma 2.1 and inequality (10) show that Lemma 2.12 is applicable to each of the diagrams $\Gamma_{1}, \Gamma_{2}$. Since $\left|s_{1}^{j}\right|<\zeta|\partial(\Pi)|$, it follows from Lemma 2.12 that an arbitrary vertex of $t_{l}^{j}(l, j \in\{1,2\})$ can be connected in $\Gamma_{j}$ with a vertex of $t_{3-l}^{j}$ by a path of length at most $3 \gamma^{-1} \zeta|\partial(\Pi)|$. Therefore, for $j=1,2$, there exist subdiagrams $\Gamma_{j}^{\prime}$ of $\Gamma_{j}$ with contours $x_{1}^{j} y_{1}^{j} x_{2}^{j} y_{2}^{j}$, where $\left|x_{l}^{j}\right|<3 \gamma^{-1} \zeta|\partial(\Pi)|$, $\phi\left(y_{2}^{1}\right) \equiv \phi\left(y_{2}^{2}\right)^{-1} \equiv U$ and $y_{1}^{j}$ are subpaths of $t_{1}^{j}$.

By gluing diagrams $\Gamma_{1}^{\prime}$ and $\Gamma_{2}^{\prime}$ along $y_{2}^{1}$ and $\left(y_{2}^{2}\right)^{-1}$, one can obtain (after deleting $j$-pairs) a greduced diagram $\Gamma_{0}$ with contour $z_{1} y_{1}^{1} z_{2} y_{1}^{2}$ where $\left|z_{1}\right|,\left|z_{2}\right|<6 \gamma^{-1} \zeta|\partial(\Pi)|$. Notice, that by property A1 and Lemma 2.7,

$$
\left|y_{1}^{j}\right| \geq \bar{\beta}\left|y_{2}^{j}\right|-\left|x_{1}^{j}\right|-\left|x_{2}^{j}\right|>\frac{1}{7} \bar{\beta}|\partial(\Pi)|-6 \gamma^{-1} \zeta|\partial(\Pi)|>\frac{1}{8}|\partial(\Pi)| \geq \frac{n}{8}|B|
$$

Therefore one can apply Lemma 2.15 to $\Gamma_{0}$ and obtain a subdiagram $\Gamma_{0}^{\prime}$ of $\Gamma_{0}$ with contour $v_{1} w_{1} v_{2} w_{2}$, where $w_{j}$ is a subpath of $y_{1}^{j}$ (for $j=1,2$ ), $\left|v_{1}\right|,\left|v_{2}\right|<\alpha|B|$, and

$$
\left|w_{1}\right|,\left|w_{2}\right|>\frac{1}{8}|\partial(\Pi)|-\gamma^{-1}\left(12 \gamma^{-1} \zeta|\partial(\Pi)|+|B|\right)>\frac{n}{9}|B|
$$

since $|\partial(\Pi)| \geq n|B|$ and the rank of $\Pi$ is $k=|B|$. Since the labels of both $w_{1}$ and $w_{2}$ are $B$-periodic words, and period $B$ is simple in rank $r\left(\Gamma_{0}^{\prime}\right)<k$, we obtain a contradiction with Lemma 3.33 in a smaller rank.

Assume now that $o_{2} \in p^{\prime \prime}$. Then we consider $o_{1}^{\prime}=o=\left(s_{2}^{2}\right)_{+}$. There is a vertex $o_{2}^{\prime}$ on the path $q_{1}$, such that $o_{2}^{\prime}-o_{1}$ is a subpath of $q_{1}$ of length $\left|o_{2}-o\right|<\frac{1}{3}|\partial(\Pi)|$. Then $o_{1}^{\prime}$ and $o_{2}^{\prime}$ are in the same phase. Thus, this case is completely analogous to the case when $o_{2} \in p^{\prime}$ up to the interchange of the paths $q_{1}$ and $q_{2}^{-1}$. The lemma is proved.

Lemma 3.22. Let $\Delta$ be a narrow contiguity diagram of rank $i$ between $q_{1}$ and $q_{2}$ with contour $p_{1} q_{1} p_{2} q_{2}$, where $q_{1}$ and $q_{2}^{-1}$ are paths with $A$-periodic labels for some simple in rank $i$ period $A$. Assume that there are vertices $o_{1}$ and $o_{2}$ on $q_{1}$ and $q_{2}$ respectively, which are in the same phase, the label $\phi\left(o_{1}-o_{2}\right)$ of a path $o_{1}-o_{2}$ is a conjugate of a 0 -word in rank $i$ and ||$\left(q_{1}\right)_{-}-o_{1}|-|\left(q_{2}\right)_{+}-o_{2}|| \leq$ $\frac{1}{3} \min \left(\left|q_{1}\right|,\left|q_{2}\right|\right)$, where $\left(q_{1}\right)_{-}-o_{1}$ and $o_{2}-\left(q_{2}\right)_{+}$are subpaths of $q_{1}$ and $q_{2}$. Then $r(\Delta)=0$.

Proof. As in the proof of Lemma 2.13, $\Delta$ can be partitioned into several narrow bonds $E_{l}$ of an $\alpha$-series and several subdiagrams $\Delta_{1}, \Delta_{2}, \ldots$ between successive bonds. If $r\left(E_{l}\right)=r\left(E_{l+1}\right)=0$, then $r\left(\Delta_{l}\right)=0$ by Lemma 2.11 and the maximality of the $\alpha$-series. Thus it suffices to prove that $r\left(E_{l}\right)=0$ for every $E_{l}$. Proving by contradiction, we choose the bond $E_{l}$ whose principal cell $\Pi_{0}$ has maximal perimeter (see Figure 9). 


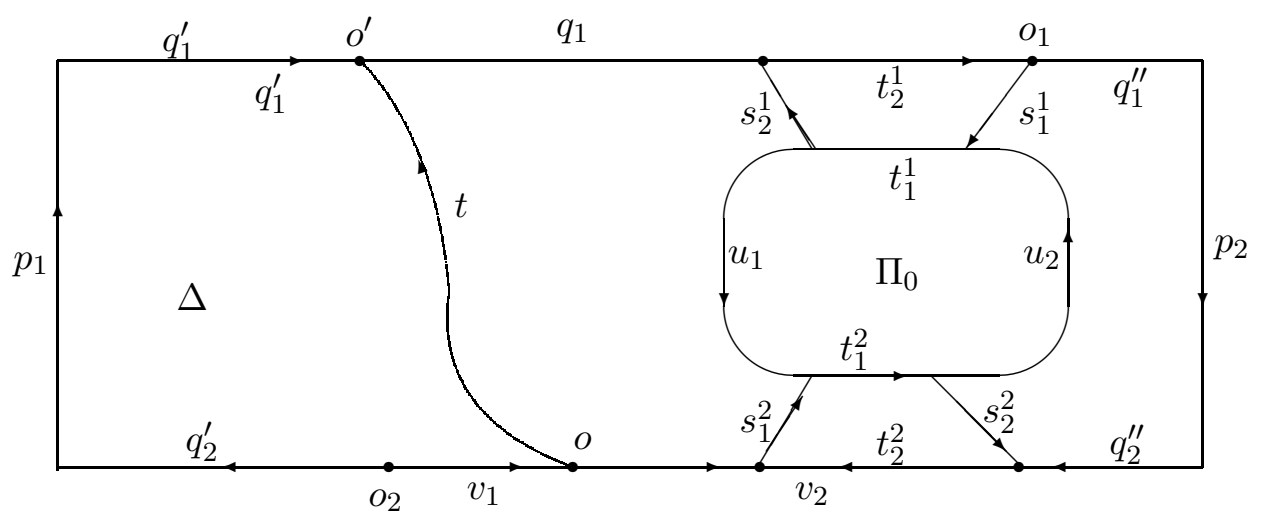

Fig. 9.

By Lemma 2.7 (and lemmas 3.38, 3.39) $\left|q_{2}\right| \leq \bar{\beta}^{-1}\left(\left|q_{1}\right|+\left|p_{1}\right|+\left|p_{2}\right|\right)$. We have $\left|p_{1}\right|+\left|p_{2}\right|<$ $(\alpha+4 \zeta)\left|\partial\left(\Pi_{0}\right)\right|$ as in Lemma 2.13 for the side arcs of narrow bonds. Denote by $\Gamma_{1}$ and $\Gamma_{2}$ the contiguity subdiagrams of $\Pi_{0}$ to $q_{1}$ and $q_{2}$ with contours $s_{1}^{j} t_{1}^{j} s_{2}^{j} t_{2}^{j}(j=1,2)$ as in Lemma 3.21. Applying lemmas $2.2,2.3$, to $\Gamma_{1}$, we have $\left|q_{1}\right|>(1-\alpha-\bar{\alpha})\left|\partial\left(\Pi_{0}\right)\right|$, because $q_{1}$ contains $t_{2}^{1}$. Hence $\left|p_{1}\right|+\left|p_{2}\right|<\frac{5}{2} \alpha\left|q_{1}\right|$ and $\left|q_{2}\right|<(1+3 \alpha)\left|q_{1}\right|$.

Then we decompose $q_{1}=q_{1}^{\prime} t_{2}^{1} q_{1}^{\prime \prime}, q_{2}=q_{2}^{\prime \prime} t_{2}^{2} q_{2}^{\prime}$ and $\partial(\Pi)=t_{1}^{1} u_{1} t_{1}^{2} u_{2}$.

Observe that since $E_{l}$ is also a bond of a narrow contiguity subdiagram between the paths $q_{1}^{\prime} t_{2}^{1}$ and $t_{2}^{2} q_{2}^{\prime}$, we have the similar inequality as for $\left|q_{1}\right|$ and $\left|q_{2}\right|$ above: $\left|q_{1}^{\prime} t_{2}^{1}\right|<(1+3 \alpha)\left|t_{2}^{2} q_{2}^{\prime}\right|$. This implies that $\left|q_{1}^{\prime}\right|+\left|q_{2}^{\prime \prime}\right|<(1+3 \alpha)\left(\left|q_{2}^{\prime}\right|+\left|t_{2}^{2}\right|+\left|q_{2}^{\prime \prime}\right|\right)=(1+3 \alpha)\left|q_{2}\right|$. Similarly, $\left|q_{1}^{\prime}\right|+\left|q_{2}^{\prime \prime}\right|<(1+3 \alpha)\left|q_{1}\right|$.

Assume first that $o_{1} \in q_{1}^{\prime}$ and $o_{2} \in q_{2}^{\prime \prime}$. We may also suppose by symmetry that $\left|q_{1}^{\prime}\right|<$ $\frac{1+3 \alpha}{2} \min \left(\left|q_{1}\right|,\left|q_{2}\right|\right)$. Notice that equal shifts of the vertices $o_{1}, o_{2}$ along $q_{1}$ and $q_{2}$ preserve the assumption of the lemma. Now assume that, after shifts, $o_{2}$ becomes equal to $\left(q_{2}\right)_{-}$and $o_{1}$ still belongs to $q_{1}^{\prime}$. The last assumption leads to a contradiction, because we have for the new $o_{1}$ and $o_{2}$ that $\left|o_{2}-\left(q_{2}\right)_{+}\right|-\left|o_{1}-\left(q_{1}\right)_{-}\right|>\left|q_{2}\right|-\frac{1+3 \alpha}{2}\left|q_{2}\right|>\left|q_{2}\right| / 3$ contrary to the assumption of the lemma. Therefore one can move $o_{1}$ to $\left(t_{2}^{1}\right)_{-}$and $o_{2}$ to a vertex laying on $q_{2}$, in this case.

If $o_{1} \in q_{1}^{\prime}$ and $o_{2} \in t_{2}^{2}\left(o_{2} \in q_{2}^{\prime}\right)$, then obviously, one can make equal shifts of these vertices so that either $o_{1}$ becomes equal to $\left(t_{2}^{1}\right)_{-}$or $o_{2}$ becomes equal to $\left(t_{2}^{2}\right)_{-}$. Similarly, one of the vertices $o_{1}, o_{2}$ can be made equal to one of the $\left(t_{2}^{1}\right)_{ \pm},\left(t_{2}^{2}\right)_{ \pm}$after parallel shifts, if $o_{1} \in t_{2}^{1}$ and $o_{2} \in t_{2}^{2}$. The remaining cases are symmetric to the examined ones.

Thus, to prove the lemma, one may assume that either $o_{1}=\left(t_{2}^{1}\right)_{-}$or $o_{1}=\left(t_{2}^{1}\right)_{+}$, or $o_{2}=\left(t_{2}^{2}\right)_{-}$, or $o_{2}=\left(t_{2}^{2}\right)_{+}$. We will assume that $o_{1}=\left(t_{2}^{1}\right)_{+}=\left(s_{1}^{1}\right)_{-}$.

In addition, one may assume that the vertex $o_{2}$ belongs to the subpath $t_{2}^{2} q_{2}^{\prime}$, because otherwise it belongs to $q_{2}^{\prime \prime}$ and $\left|o_{2}-\left(t_{2}^{2}\right)_{-}\right| \geq \frac{1}{3}\left|\partial\left(\Pi_{0}\right)\right|$ by Lemma 3.21. In this case, by Lemma 2.2 , one might shift $o_{1}$ to $\left(t_{2}^{1}\right)_{-}$so that corresponding vertex $o_{2}$ shifts to a vertex of the path $q_{2}^{\prime \prime} t_{2}^{2}$ because

$$
\begin{aligned}
& \left|o_{2}-\left(t_{2}^{2}\right)_{+}\right|>\frac{1}{3}\left|\partial\left(\Pi_{0}\right)\right|+\left|t_{2}^{2}\right|>(1 / 3+(1-2 \beta)(1-\alpha-\bar{\alpha}))\left|\partial\left(\Pi_{0}\right)\right|> \\
& \frac{2}{3}\left|\partial\left(\Pi_{0}\right)\right|>(1+2 \beta) \bar{\alpha}\left|\partial\left(\Pi_{0}\right)\right|>\left|t_{2}^{1}\right|
\end{aligned}
$$

Then one gets a situation which is symmetric to the one considered above with respect to changing of notations $p_{1} \rightarrow p_{2}^{-1}, p_{2} \rightarrow p_{1}^{-1}, q_{1} \rightarrow q_{1}^{-1}, q_{2} \rightarrow q_{2}^{-1}$ (i.e. we replace $\Delta$ by its mirror copy).

Thus, up to symmetries, one has to consider only one case when $o_{1}=\left(t_{2}^{1}\right)_{+}$and $o_{2} \in t_{2}^{2} q_{2}^{\prime}$. The vertices $o_{2}$ and $o_{1}$ can be connected by a path $v u$, where $v=o_{2}-\left(t_{2}^{2}\right)_{-}$and $u=\left(s_{2}^{2}\right)^{-1} u_{2}\left(s_{1}^{1}\right)^{-1}$. Set $V \equiv \phi(v)$ and $U \equiv \phi(u)$. As has been mentioned, $|U| \leq(\alpha+2 \zeta)\left|\partial\left(\Pi_{0}\right)\right|$. On the other hand, 
$|V| \geq \frac{1}{3}\left|\partial\left(\Pi_{0}\right)\right|$ by Lemma 3.21. Therefore one gets the decomposition $V \equiv V_{1} V_{2}$ guaranteed by Lemma 3.20. Denote by $o$ the vertex of $v$ which gives the corresponding decomposition $v=v_{1} v_{2}$. There are two subcases.

(1) $|V|<3 \alpha^{-1}\left|\partial\left(\Pi_{0}\right)\right|$. Recall, that by Lemma 3.20 the word $V_{2} U V_{1}$ is equal in rank $i$ to a word $S$ of length at most $3 \gamma|V|$ and

$$
|| V_{1}|-| V_{2}||<\beta|V|+|U|<\left(3 \alpha^{-1} \beta+\alpha+2 \zeta\right)\left|\partial\left(\Pi_{0}\right)\right|<2 \alpha\left|\partial\left(\Pi_{0}\right)\right| .
$$

By Lemma 3.21, $|V| \geq\left|\partial\left(\Pi_{0}\right)\right| / 3$, whence $\left|V_{1}\right|,\left|V_{2}\right|>(1 / 6-\alpha)\left|\partial\left(\Pi_{0}\right)\right|>1 / 7\left|\partial\left(\Pi_{0}\right)\right|$ by (12). Since

$$
0.45\left|\partial\left(\Pi_{0}\right)\right|<(1+2 \beta)^{-1}(1-\bar{\alpha}-\alpha)\left|\partial\left(\Pi_{0}\right)\right|<\left|t_{2}^{j}\right|<(1-2 \beta)^{-1} \bar{\alpha}\left|\partial\left(\Pi_{0}\right)\right|
$$

by lemmas 2.3 and 2.2 , we have in case $o \in t_{2}^{2}$ that

$$
\left|o-\left(s_{1}^{2}\right)_{-}\right|<\left|t_{2}^{2}\right|-\left|V_{2}\right|<((1+2 \beta) \bar{\alpha}-1 / 6+\alpha)\left|\partial\left(\Pi_{0}\right)\right|<0.35\left|\partial\left(\Pi_{0}\right)\right|
$$

If $o \in q_{2}^{\prime}$, then

$$
\left|o-\left(s_{1}^{2}\right)_{-}\right|=\left|V_{2}\right|-\left|t_{2}^{2}\right|<\left|V_{1}\right|+2 \alpha\left|\partial\left(\Pi_{0}\right)\right|-0.45\left|\partial\left(\Pi_{0}\right)\right| .
$$

Hence in any case the path between $o$ and $\left(s_{1}^{2}\right)_{-}$has length at most $\max \left(0.35\left|\partial\left(\Pi_{0}\right)\right|,\left|V_{1}\right|-\right.$ $\left.2 \alpha\left|\partial\left(\Pi_{0}\right)\right|\right)$, because of $(12)$ and the inequality $4 \alpha<0.45$. Since $\left|s_{1}^{2} u_{1}^{-1} s_{2}^{1}\right|<(\alpha+2 \zeta)\left|\partial\left(\Pi_{0}\right)\right|$, the vertex $o$ can be connected with $\left(t_{2}^{1}\right)_{-}$by a path $p$ of length at most $\max \left(0.4\left|\partial\left(\Pi_{0}\right)\right|,\left|V_{1}\right|\right)$.

The path $p t_{2}^{1}$ is homotopic to the path $v_{2} u$. Thus the word $S$ is equal in rank $i$ to the word $P \phi\left(t_{2}^{1}\right) V_{1}$, where $P \equiv \phi(p)$. The word $W \equiv \phi\left(t_{2}^{1}\right) V_{1}$ is A-periodic because the vertices $o_{2}$ and $o_{1}$ are in the same phase. It has length at least $\left|V_{1}\right|+\left|t_{2}^{1}\right|>\left|V_{1}\right|+0.45\left|\partial\left(\Pi_{0}\right)\right|$. Then $W=P^{-1} S$ in rank $i$, which implies, by lemmas 2.7 and 3.38, 3.39 applied to the diagram for this equality, that

$$
\max \left(0.4\left|\partial\left(\Pi_{0}\right)\right|,\left|V_{1}\right|\right)+3 \gamma|V|>|P|+|S|>\bar{\beta}\left(\left|V_{1}\right|+\left|t_{2}^{1}\right|\right)>\bar{\beta}\left(\left|V_{1}\right|+0.45\left|\partial\left(\Pi_{0}\right)\right|\right)
$$

On the other hand, since $\left|V_{1}\right| \leq|V| \leq 3 \alpha^{-1}\left|\partial\left(\Pi_{0}\right)\right|$, we have $(\beta+3 \gamma)|V|<(0.45 \bar{\beta}-0.4)\left|\partial\left(\Pi_{0}\right)\right|$ which contradicts inequality (13).

(2) Let $|V| \geq 3 \alpha^{-1}\left|\partial\left(\Pi_{0}\right)\right|$. Recall that $\left|V_{1}\right|-\left|V_{2}\right|<\beta|V|+(\alpha+2 \zeta)\left|\partial\left(\Pi_{0}\right)\right|$, and since $|V|=\left|V_{1}\right|+$ $\left|V_{2}\right| \geq 3 \alpha^{-1}\left|\partial\left(\Pi_{0}\right)\right|$, the length of $v_{2}$ is greater than $\frac{7}{15}|V| \geq 1.4 \alpha^{-1}\left|\partial\left(\Pi_{0}\right)\right|$. By Lemma 2.13, the vertex $o$ can be connected with a vertex $o^{\prime} \in q_{1}^{\prime} t_{2}^{1}$ by a path $t$ of length at most $\alpha^{-1}\left|\partial\left(\Pi_{0}\right)\right|<\frac{1}{3}|V|$. By Lemma 2.7, the length of the path $q=o^{\prime}-o_{1}$ is at least

$$
\bar{\beta}\left|v_{2}\right|-|t|-|u|>\frac{7}{15}|V|-\frac{1}{3}|V|-\alpha^{2}|V|>|V| / 8
$$

because $|u| \leq(\alpha+2 \zeta)\left|\partial\left(\Pi_{0}\right)\right|<\alpha^{2}|V|$ by the assumption of case (2). Therefore the length of the $A$-periodic word $W \equiv Q V_{1}$ (where $Q \equiv \phi(q)$ ) is greater than $\left(\frac{7}{15}+\frac{1}{8}\right)|V| \geq \frac{7}{12}|V|$. By Lemma 2.7, $W$ cannot be equal in rank $i$ to a word of length $\leq|V| / 2$. But it is equal in rank $i$ to the label of $t$ times $S$ which is equal in rank $i$ to $V_{2} U V_{1}$. Therefore $W$ is equal in rank $i$ to a word of length $<\left(\frac{1}{3}+3 \gamma\right)|V|$, a contradiction.

Lemma 3.23. Assume that $A$ is a word which is not a conjugate in rank $i$ of a 0-word. Let $A=X B^{l} R X^{-1}$ in rank $i$, where $B$ is a simple in rank $i$ word or a period of rank $j \leq i$ and $R \in \mathbf{0}(B)$. Then $\mathbf{0}(A) \leq X \mathbf{0}(B) X^{-1}$ in rank $i$ (i.e. the canonical image of $\mathbf{0}(A)$ in $\mathbf{G}(i)$ is a subgroup of the image of $\left.X \mathbf{0}(B) X^{-1}\right)$. 
Proof. One may suppose that $A$ is a $Y$-reduced word, moreover that $A$ is cyclically $Y$-reduced, by Lemma 3.4(1) and Lemma 3.7.

Consider a diagram $\Delta_{1}$ of rank $i$ for the equality $A=X B^{l} R X^{-1}$. Let $\Delta_{2}$ be an annular diagram which arises after identifying the boundary sections of $\Delta_{1}$ labeled by $X$. It has contours $q_{1}$ and $q_{2}$ labeled by $A$ and $B^{l} R$, respectively. Initial vertices $o_{1}$ of $q_{1}$ and $o_{2}$ of $q_{2}$ are connected in $\Delta_{2}$ by a path $x$ labeled by the word $X$. If $\Delta_{2}$ is not g-reduced, one can reduce it and obtain a diagram $\Delta_{3}$ of a smaller type in which the vertices $o_{1}$ and $o_{2}$ are connected by a path $x^{\prime}$ with $X^{\prime} \equiv \phi\left(x^{\prime}\right)=X$ in rank $i$. (Lemma 11.3 [30].) We will induct on the type of $\Delta_{3}$.

If $r\left(\Delta_{3}\right)=0$, then $\mathbf{0}(A)=X^{\prime} \mathbf{0}\left(B^{l} R\right)\left(X^{\prime}\right)^{-1}$ in rank 0, by Lemma 3.7. Since $X^{\prime}=X$ in rank $i$, the statement of the lemma follows from Lemma 3.11.

Assume now that $r\left(\Delta_{3}\right)>0$. One may suppose that there are no cells compatible with $q_{2}$ in $\Delta_{3}$ (if $B$ is a period of rank $j \leq i$ ), since otherwise one can decrease the type of $\Delta_{3}$ by Lemma 3.18 and replace the label of $q_{2}$ for $B^{\ell \pm s n} R$. Therefore the cyclic section $q_{2}$ is smooth by Lemma 3.39, and so the degree of contiguity of any cell of $\Delta_{3}$ to $q_{2}$ is less $\bar{\alpha}$ by Lemma 2.3. By Lemma 2.4 there is a positive cell $\Pi$ and its contiguity subdiagram $\Gamma^{\prime}$ to $q_{1}$ such that $\left(\Pi, \Gamma^{\prime}, q_{1}\right)>\frac{1}{2}(\bar{\gamma}-\bar{\alpha})>\varepsilon$.

It follows, from Lemma 2.6 that there is a positive cell $\pi$ of $\Delta_{3}$ and a contiguity subdiagram $\Gamma$ of rank 0 of $\pi$ to $q_{1}$, such that $\left(\pi, \Gamma, q_{1}\right) \geq \varepsilon$.

Let $\partial\left(\pi, \Gamma, q_{1}\right)=s_{1} t_{1} s_{2} t_{2}$. Then $\left|s_{1}\right|=\left|s_{2}\right|=0$ since $r(\Gamma)=0$. There are no $Y$-bands connecting different edges of subpath $t_{1}$ of $\partial(\pi)$ by axiom A2. Therefore $\left|t_{1}\right| \leq\left|t_{2}\right|$, and the word $A^{\prime}$ obtained by the exchange in $A$ of the subword $\phi\left(t_{2}\right)$ by $\phi\left(s_{2} t_{1} s_{1}\right)^{-1}$, is $Y$-reduced (and equal to $A$ in rank 0.)

The subword $\phi\left(t_{1}\right)^{-1}$ of $A^{\prime}$ is a subword of the boundary label of $\pi$ and $\left|\phi\left(t_{1}\right)^{-1}\right| \geq \varepsilon|\partial(\pi)|$. By Lemmas 3.15, 3.16, if one substitutes $\phi\left(t_{1}\right)^{-1}$ in $A^{\prime}$ by $\phi(t)$, where $t_{1} t$ is the contour of $\pi$, and replaces the resulting word by an equal in rank $0 Y$-reduced word $A^{\prime \prime}$, then $\mathbf{0}\left(A^{\prime \prime}\right) \geq \mathbf{0}\left(A^{\prime}\right)=\mathbf{0}(A)$.

The word $A^{\prime \prime}$ is the label of the contour of a g-reduced diagram $\Delta_{4}$ which is obtained from $\Delta_{3}$ by cutting the cell $\pi$ out and (possibly) adding several 0-cells. Of course, such surgeries (cutting off a contiguity diagram or a cell) can touch the path $x^{\prime}$, but there is a homotopic path $x^{\prime \prime}$ with label $X^{\prime \prime}=X^{\prime}=X$ in rank $i$ which is not touched by the surgeries (i.e. this path does not pass through the contiguity subdiagram and does not have common edges with the cell). By the inductive hypothesis for the diagram $\Delta_{4}$ of a smaller type, $\mathbf{0}\left(A^{\prime \prime}\right) \leq X^{\prime \prime} \mathbf{0}(B)\left(X^{\prime \prime}\right)^{-1}$ in rank $i$. Hence $\mathbf{0}(A) \leq X \mathbf{0}(B) X^{-1}$, as desired.

We need the statement of the following lemma to define periods of rank $i_{+}$.

Lemma 3.24. The following relation $\sim_{i}$ is an equivalence on the set of all simple in rank $i$ words: $A \sim_{i} B$ by definition, if there are words $X, P, R$, where $P \in \mathbf{0}(A), R \in \mathbf{0}(B)$ (in rank 0 ), such that $A P=X B^{ \pm 1} R X^{-1}$ in rank $i$.

Proof. Obviously, $\sim_{i}$ is a reflexive relation.

The equality $A P=X B^{ \pm 1} R X^{-1}$ implies $B^{ \pm 1} R=X^{-1} A P X$ in rank $i$. Taking the inverses, if necessary, we have equality $B R_{ \pm}=X^{-1} A^{ \pm 1} P_{ \pm} X$ for a suitable $P_{ \pm} \in \mathbf{0}(A), R_{ \pm} \in \mathbf{0}(B)$, because $\mathbf{0}(A)$ and $\mathbf{0}(B)$ are normalized by $A$ and $B$ respectively. By Lemma $3.9 \mathbf{0}(B)=\mathbf{0}\left(B R_{ \pm}\right)$, and so $R_{ \pm} \in X^{-1} \mathbf{0}(A) X$ by Lemma 3.23. Then $Q \equiv X R_{ \pm} X^{-1} \in \mathbf{0}(A)$ by Lemma 3.7. Hence $B=$ $X^{-1} A^{ \pm 1} V X$ in rank $i$, where $V \equiv P_{ \pm} Q \in \mathbf{0}(A)$, and so the relation $\sim_{i}$ is symmetric.

We have also proved that $B \sim_{i} A$ implies that $B=X^{-1} A^{ \pm 1} V X$ in rank $i$ for some $V \in \mathbf{0}(A)$ and a word $X$. 
Assume now that $B \sim_{i} C$, that is $B=Y C^{ \pm 1} Q Y^{-1}$ in rank $i$ for a word $Y$ and some $Q \in \mathbf{0}(C)$. Then, in rank $i, A P=X\left(Y C^{ \pm 1} Q_{ \pm} Y^{-1}\right) R X^{-1}$ for suitable $Q_{ \pm} \in \mathbf{0}(C)$, and $V \equiv Y^{-1} R Y \in \mathbf{0}(C)$ by lemmas 3.23 and 3.7. Hence, in rank $i, A P=(X Y) C^{ \pm 1}\left(Q_{ \pm} V\right)(X Y)^{-1}$, where $Q_{ \pm} V \in \mathbf{0}(C)$. Therefore the relation $\sim_{i}$ is transitive.

Lemma 3.25. Every word $X$ is a conjugate in rank $i \geq 0$ either of a 0 -word or of a word $A^{l} P$, where $|A| \leq|X|$, $A$ is either period of rank $j \leq i$ or a simple in rank $i$ word and $P$ represents in rank 0 an element of the subgroup $\mathbf{0}(A)$.

Proof. If the statement were false for $X$, then, in rank $i, X=Y A^{s} R Y^{-1}$ for some ( $Y$-reduced) essential word $A$ with $|A|<|X|$ and word $R \in \mathbf{0}(A)$. By the inductive hypothesis, we may assume that $A=Z B^{t} P Z^{-1}$ in rank $i$ where $B$ is either a period of rank $j \leq i$ or a simple in rank $i$ word, and $P \in \mathbf{0}(B)$.

We have that $A^{s} R=Z\left(B^{t} P\right)^{s} Z^{-1} R$ in rank $i$. The word $Z^{-1} R Z$ is equal in rank $i$ to a word $Q \in \mathbf{0}\left(B^{t} P\right)=\mathbf{0}(B)$ by Lemma 3.23 and Lemma 3.11. Therefore $A^{s} R=Z\left(\left(B^{t} P\right)^{s} Q\right) Z^{-1}$. Since $\mathbf{0}(B)$ is normalized by $B$ (by definition), $X$ is a conjugate in rank $i$ of a word of the form $B^{s t} S$ for some $S \in \mathbf{0}(B)$, as desired.

Lemma 3.26. If a word $X$ has a finite order in rank $i$, then it is a conjugate in rank $i$ either of a 0 -word or of a word $A^{l} R$, where $A$ is a period of rank $j \leq i$ and $R \in \mathbf{0}(A)$.

Proof. If the claim were false, then, by Lemma 3.25, since $\mathbf{0}(A)$ is normalized by $A, A^{l} R=1$ in rank $i$ for some simple in $\operatorname{rank} i$ word $A, R \in \mathbf{0}(A)$ and $|l|>0$. Then $A^{l}=R^{-1}$ in rank $i$ and $\bar{\beta}|l||A|<|R|=0$ by lemmas 3.38, 3.39 and Lemma 2.7. The statement is proved by contradiction.

Lemma 3.27. If $A$ and $B$ are simple in rank $i$ words and $A$ is equal in rank $i$ to $X B^{l} R X^{-1}$ for some word $X$, and some word $R \in \mathbf{0}(B)$, then $l= \pm 1$ and $|A|=|B|$.

Proof. When proving the first assertion by contradiction, one can assume that $l \geq 2$. By Lemma 3.39 and Lemma 2.7 for a g-reduced annular diagram for the conjugacy of $A$ and $B^{l} R$, we have $2 \bar{\beta}|B|<|A|$, whence $|B|<|A|$ contrary to the simplicity of the word $A$ in rank $i$. Then we have $P \equiv X R X^{-1} \in \mathbf{0}(A)$ in rank $i$ by Lemma 3.7, Lemma 3.11, and $B=X^{-1}\left(A P^{-1}\right)^{ \pm 1} X$. Hence $|A|=\left|A P^{-1}\right| \geq|B|$ since $|P|=0$ by definition of $\mathbf{0}(A)$ (see property (Z3)) and the simplicity of $B$ in rank $i$.

Lemma 3.28. If words $X$ and $Y$ are conjugate in rank $i$, and $X$ is not a conjugate in rank $i$ of a 0 -word, then there is a word $Z$, such that $|Z| \leq \bar{\alpha}(|X|+|Y|)$ and $Z Y Z^{-1}$ is equal to $X$ in rank $i$.

Proof. The proof is analogous to the proof of Lemma 18.5 [30]. One may apply Lemma 2.16 (which is an analog of Lemma 17.1 [30]) since $X$ is not a conjugate of a 0 -word.

Lemma 3.29. Let $\Delta$ be a narrow contiguity diagram between sections $q_{1}$ and $q_{2}$ of its contour. Assume that it is of rank 0 and has contour $p_{1} q_{1} p_{2} q_{2}$, where $q_{1}$ and $q_{2}^{-1}$ are paths labeled by $A$ periodic words with a simple in rank $i$ period $A$. Assume that $\left|q_{1}\right|,\left|q_{2}\right| \geq 2|A|$, and $o_{1}$ and $o_{2}$ are vertices of $q_{1}$ and $q_{2}$ which are in the same phase, $\left|\left(q_{1}\right)_{-}-\left(o_{1}\right)\right| \leq\left|q_{1}\right| / 2,\left|\left(q_{2}\right)_{+}-\left(o_{2}\right)\right| \leq\left|q_{2}\right| / 2$, and $\phi\left(o_{1}-o_{2}\right)$ is a conjugate in rank $i$ of a 0 -word. Then the vertices $\left(q_{1}\right)_{-}$and $\left(q_{2}\right)_{+}$are in the same phase too. 
Proof. Without loss of generality, one may assume that $\left(q_{2}\right)_{+}=o_{2}$ since the vertices $o_{1}$ and $o_{2}$ can be simultaneously shifted along the paths $q_{1}$ and $q_{2}$.

Every $Y$-band of $\Delta$ is a 0 -bond between $q_{1}$ and $q_{2}$ because the narrow diagram $\Delta$ of rank 0 is defined by 0 -bonds and by Lemma 3.2, there are no bands connecting different $Y$-edges of $q_{1}$ (of $q_{2}$ ) as the period $A$ is simple in rank 0 . Hence $\left|p_{1}\right|=0$, and the vertex $o_{1}$ can be connected with a vertex $o_{0}$ of $q_{2}$ by a path of length 0 . Proving by contradiction, one may suppose that $\left|V^{\prime}\right|>0$ for the word $V^{\prime} \equiv \phi\left(\left(q_{1}\right)_{-}-o_{1}\right)$. Then $|V|=\left|V^{\prime}\right|>0$ for the word $V \equiv \phi\left(o_{2}-o_{0}\right)$.

Denote $U \equiv \phi\left(o_{0}-o_{1}\right)$. Then $|U|=0$, and the word $V U$ is a conjugate of a 0 -word in rank $i$ by the hypothesis of the lemma. Lemma 3.20 provides us with a decomposition $V \equiv V_{1} V_{2}$, where $V_{2} U V_{1}$ is equal in rank 0 to a word $S$ of length at most $3 \gamma|V|$.

Consider the vertex $o$ of the subpath $o_{2}-o_{0}$ that defines the decomposition $V_{1} V_{2}$ of the label $V$ of $o_{2}-o$. Then the word $V_{2} U V_{1}$ is readable in $\Delta$ starting with $o$ : the suffix $V_{1}$ can be read in the beginning of $o_{1}-\left(q_{1}\right)_{+}$since $o_{1}$ and $o_{2}$ are in the same phase. The vertex $o$ can be connected with a vertex $o^{\prime}$ of $q_{1}$ by a path of length 0 (see Figure 10).

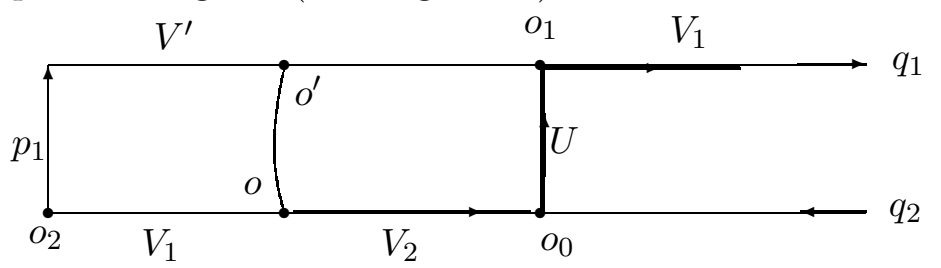

Fig. 10 .

Thus, up to a factor of length 0 , the word $V_{2} U V_{1}$ is equal to a subword of $q_{1}$ of the length $\left|V_{2}\right|+\left|V_{1}\right|=|V|$, which is not equal in rank $i$ to a word of length $\leq \bar{\beta}|V|$, by Lemma 3.39 and Lemma 2.7. The inequality $\bar{\beta}|V| \leq 3 \gamma|V|$ contradicts the Lowest Parameter Principle. This completes the proof of the lemma.

The following lemmas 3.30-3.32 are analogous to lemmas 18.6 - 18.8 [30]. For a given rank $i \geq 1 / 2$, they are proved by simultaneous induction on the sum $L$ of lengths of periods.

Lemma 3.30. Let $\Delta$ be a g-reduced diagram of rank $i$ with contour $p_{1} q_{1} p_{2} q_{2}$, where $\phi\left(q_{1}\right)$ and $\phi\left(q_{2}\right)^{-1}$ are periodic words with a simple in rank $i$ period $A$. Then the paths $q_{1}$ and $q_{2}$ are $A$ compatible in $\Delta$, provided $\left|p_{1}\right|,\left|p_{2}\right|<\alpha|A|$ and $\left|q_{1}\right|,\left|q_{2}\right|>\left(\frac{5}{6} h+1\right)|A|$. (The inductive parameter $L$ is equal to $|A|+|A|$.)

Proof. The same argument, as in the proof of Lemma 18.6 [30] allows us to assume that the vertices $\left(p_{1}\right)_{+}$and $\left(p_{1}\right)_{-}$are in the same phase, $\left|q_{1}\right|,\left|q_{2}\right|>\frac{5}{6} h|A|$ and $\left|p_{1}\right|<(\alpha+1 / 2)|A|=\bar{\alpha}|A|$. In view of Lemma 3.7, to prove the lemma, one may replace period $A$ by a cyclic permutation. Therefore we suppose that both $q_{1}$ and $q_{2}^{-1}$ start with the prefix $A$.

Suppose that the word $P_{1} \equiv \phi\left(p_{1}\right)$ is not a conjugate in rank $i$ of a 0 -word. In this case the annular diagram $\bar{\Delta}$ obtained after identification of the the long subpaths of $q_{1}$ and $q_{2}$ with equal labels, has no paths of length 0 surrounding the hole. Therefore we can apply Lemma 2.16 (which is Lemma 17.1 from [30]). The rest of the proof of Lemma 18.6 from [30] carries out with one minor change: by Lemma 3.25 , the word $D \equiv \phi\left(p_{1}\right)$ is equal in rank $i$ to the product $Y B^{m} R Y^{-1}$, $R \in \mathbf{0}(B)$ instead of simply $Y B^{m} Y^{-1}$. The word $D^{l}$ is equal in rank $i$ to $Y B^{m l} Q Y^{-1}$, for some 
0 -word $Q \in \mathbf{0}(B)$ since $\mathbf{0}(B)$ is normalized by $B$. Then we have to define $Z \equiv Q Y^{-1} \phi\left(s^{\prime}\right)$, instead of $Z \equiv Y^{-1} \phi\left(s^{\prime}\right)$ in [30]. This does not effect on the further proof because $\left|Q^{l}\right|=0$ by the definition of $\mathbf{0}(B)$.

Assume now that the word $P$ is a conjugate in rank $i$ of a 0 -word. Since $q_{1}$ and $q_{2}$ are long enough $\left(h=\delta^{-1}\right)$, we can excise a narrow contiguity subdiagram $\Delta^{0}$ of $\Delta$ with contour $p_{1}^{\prime} q_{1}^{\prime} p_{2}^{\prime} q_{2}^{\prime}$ according to Lemma 2.11 (which can be applied because $q_{1}$ and $q_{2}$ are smooth sections of rank $|A|$ by Lemma 3.39 in the smaller rank). Moreover by Lemma 2.11, we may suppose that $\left|q_{1}^{\prime}\right|,\left|q_{2}^{\prime}\right|>\frac{1}{2} h|A|$ and $\left|p_{1}^{\prime}\right|,\left|p_{2}^{\prime}\right|<\alpha|A|$. Again, by Lemma 2.11, there are vertices $o_{1}$ and $o_{2}$ of $q_{1}^{\prime}$ and $q_{2}^{\prime}$ respectively, such that the paths $\left(p_{1}\right)_{+}-o_{1}$ and $\left(p_{1}\right)_{-}-o_{2}$ have the same label (say, $X$ ) and

$$
\left|\left(q_{1}^{\prime}\right)_{-}-o_{1}\right|,\left|\left(q_{2}^{\prime}\right)_{+}-o_{2}\right|<\gamma^{-1}(\bar{\alpha}+\alpha)|A|<\frac{h}{6}|A| .
$$

Then the label of any path $o_{1}-o_{2}$ is equal in rank $i$ to $X^{-1} P^{-1} X$, and therefore it is a conjugate in rank $i$ of a 0 -word. By Lemma 3.22, $r\left(\Delta^{0}\right)=0$, and the vertices $\left(q_{1}^{\prime}\right)_{-}$and $\left(q_{2}^{\prime}\right)_{+}$are in the same phase by Lemma 3.29 (see Figure 11).

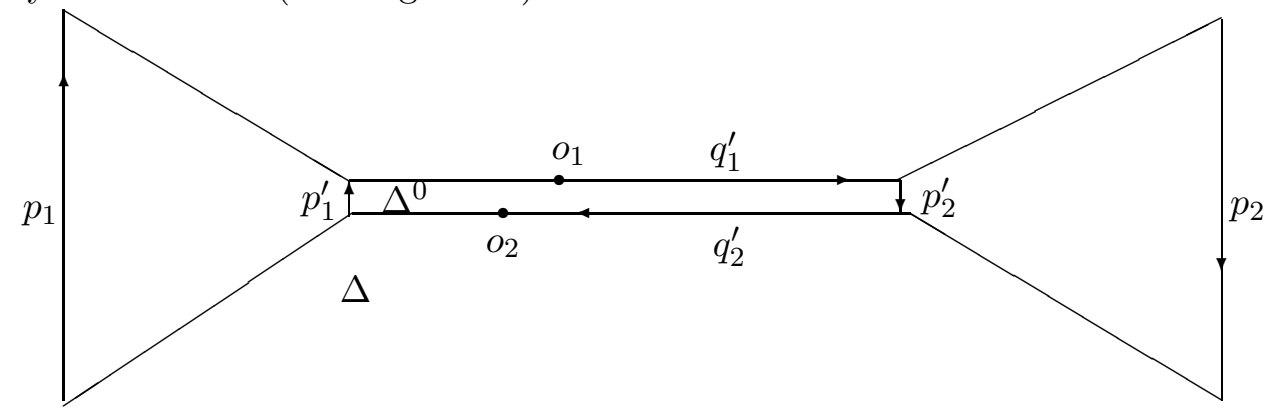

Fig. 11 .

Every $Y$-band starting on $q_{1}^{\prime}$ must end on $q_{2}^{\prime}$ by Lemma 3.2. Hence one may suppose that $\phi\left(q_{1}^{\prime}\right) \equiv \phi\left(q_{2}^{\prime}\right)^{-1} \equiv U$ for some word $U$. Then $U^{-1} \phi\left(p_{1}^{\prime}\right) U=\phi\left(p_{2}^{\prime}\right)^{-1}$ in $\mathbf{G}(0)$, and since $|U|>$ $\frac{h}{2}|A|>4|A|$, we have $A_{1}^{-4} \phi\left(p_{1}\right) A_{1}^{4} \in \mathbf{0}\left(A_{1}\right)$ by Lemma 3.6 , where $A_{1}$ is the cyclic shift of $A$ which is the prefix of $\phi\left(q_{1}^{\prime}\right)$. Thus the paths $q_{1}^{\prime}$ and $q_{2}^{\prime}$ are compatible by (Z3.1) since $A$ is simple in rank $1 / 2$ (recall that $i \geq 1 / 2$ ). Therefore the paths $q_{1}, q_{2}$ are also compatible by Lemma 3.7, and the lemma is proved.

Lemma 3.31. Let $Z_{1} A^{m_{1}} Z_{2}=A^{m_{2}}$ in rank $i, m=\min \left(m_{1}, m_{2}\right)$, and $A$ be a simple word in rank $i$. Then each of the words $Z_{1}, Z_{2}$ is equal in rank $i$ to a product of a power of $A$ and a word representing an element of the subgroup $\mathbf{0}(A)$ provided $\left|Z_{1}\right|+\left|Z_{2}\right|<\left(\gamma\left(m-\frac{5}{6} h-1\right)-1\right)|A|$. (The inductive parameter $L$ is equal to $|A|+|A|$.)

Proof. As in Lemma 18.7 [30] we can consider a g-reduced disc diagram $\Delta$ of rank $i$ with the contour $p_{1} q_{1} p_{2} q_{2}$, where $\phi\left(q_{1}\right) \equiv A^{m_{1}}, \phi\left(q_{2}^{-1}\right) \equiv A^{m_{2}}, \phi\left(p_{1}\right)=Z_{1}, \phi\left(p_{2}\right)=Z_{2}$. By lemmas 3.38, 3.39, $q_{1}$ and $q_{2}$ are smooth of rank $|A|$. By lemmas 3.38 and 2.15, we can excise from $\Delta$ a subdiagram $\Delta^{\prime}$ with contour $p_{1}^{\prime} q_{1}^{\prime} p_{2}^{\prime} q_{2}^{\prime}$ where

$$
\begin{aligned}
& \left|p_{1}^{\prime}\right|,\left|p_{2}^{\prime}\right|<\alpha|A|,\left|q_{j}^{\prime}\right|>\left|q_{j}\right|-\gamma^{-1}\left(\left|Z_{1}\right|+\left|Z_{2}\right|+|A|\right)> \\
& m|A|-\left(m-\frac{5}{6} h-1\right)|A|=\left(\frac{5}{6} h+1\right)|A|, j=1,2 .
\end{aligned}
$$


By Lemma 3.30, $q_{1}^{\prime}$ and $q_{2}^{\prime}$ are compatible in $\Delta^{\prime}$. So there exist phase vertices $o_{1} \in q_{1}$ and $o_{2} \in q_{2}$ such that the label $T=\phi(t)$ of a path $t$ connecting these vertices represents an element of $\mathbf{0}(A)$ in rank $i$. Then $\phi\left(\left(q_{1}\right)_{-}-o_{1}\right)$ and $\phi\left(\left(q_{2}\right)_{+}-o_{2}\right)$ are powers of $A$, we have an equality of the following form in rank $i$ : $Z_{1}=A^{s_{1}} T A^{s_{2}}$. Since $\mathbf{0}(A)$ is normalized by $A$ in rank 0 , the lemma is proved.

Lemma 3.32. Let $\Delta$ be a g-reduced disc diagram of rank $i$ with the contour $p_{1} q_{1} p_{2} q_{2}$, where $\phi\left(q_{1}\right)$ and $\phi\left(q_{2}\right)$ are periodic words with simple in rank $i$ periods $A$ and $B$, respectively, and $|A| \geq|B|$. Assume that $\left|p_{1}\right|,\left|p_{2}\right|<\alpha|B|,\left|q_{1}\right|>\frac{3}{4} h|A|$ and $\left|q_{2}\right|>h|B|$. Then the word $A$ is a conjugate in rank $i$ of a product $B^{ \pm 1} R$, where $R$ represents an element of the subgroup $\mathbf{0}(B)$, and $|A|=|B|$. Moreover, if the words $\phi\left(q_{1}\right)$ and $\phi\left(q_{2}\right)^{-1}$ start with $A$ and $B^{-1}$, respectively, then $A$ is equal in rank $i$ to $\phi\left(p_{1}\right)^{-1} B^{ \pm 1} R \phi\left(p_{1}\right)$. (The inductive parameter $L$ is equal to $|A|+|B|$.)

Proof. The proof is similar to the proof of Lemma 18.8 [30], but, at the very end, by Lemma 3.31, we obtain the equality $Z_{1}=B^{s} R$ (instead of $\left.Z_{1}=B^{s}\right)$ with $R \in \mathbf{0}(B)$. Then $s= \pm 1$ and $|A|=|B|$ by Lemma 3.27 .

Lemma 3.33. Let $\Delta$ be a disc diagram of rank $i$ with the contour $p_{1} q_{1} p_{2} q_{2}$, where both $\phi\left(q_{1}\right)$ and $\phi\left(q_{2}\right)$ are periodic words with a simple in rank $i$ period $A$ and $\left|p_{1}\right|,\left|p_{2}\right|<\alpha|A|$. Then $\left|q_{1}\right|,\left|q_{2}\right| \leq h|A|$.

Proof. We prove the statement by contradiction. The proof is parallel to the proof of Lemma 18.9 [30] up to the application of Lemma 3.32 (instead of Lemma 18.8). This lemma gives the equality $A R=Z^{-1}\left(A^{-1}\right)^{l} Z$ in $\operatorname{rank} i$, where $l= \pm 1, R \in \mathbf{0}(A),|Z|<(1+\alpha)|A|$. Then, in case $l=-1$, $Z^{-1} A^{k} Z=A^{k} Q$ in rank $i$, where $Q \in \mathbf{0}(A)$, because of (Z3) where $\phi\left(q_{1}\right) \equiv A^{k} \bar{A},|\bar{A}|<|A|$. The rest of the argument of the proof of Lemma 18.9 from [30] in this case remains valid because $\mathbf{0}(A)$ is normalized by $A$ in rank 0 and all elements in $\mathbf{0}(A)$ have length 0 (so the corresponding factors do not affect length estimates).

In case $l=1$, the equality $A R=Z^{-1} A^{-1} Z$, in rank $i$, implies $A^{n}=Z^{-1} A^{-n} Z$ by Lemma 3.12. Hence $Z^{2}$ commutes with $A^{n}$ in rank $i$. Therefore $Z^{2}$ commutes with any $A^{s n}, s=1,2, \ldots$. Taking $s$ sufficiently large, we can apply Lemma 3.31 and conclude that $Z^{2}=A^{d} P$ in rank $i$, with $P \in \mathbf{0}(A)$. Then again, $Z^{2 n}=A^{d n}$ in rank $i$ by Lemma 3.12. Therefore the word $Z$ commutes with $A^{d n}$ in rank $i$. But the equality $A^{n}=Z^{-1} A^{-n} Z$ implies that $Z^{-1} A^{d n} Z=A^{-d n}$ in rank $i$. Hence $A^{2 d n}=1$ in rank $i$. This would contradict the definition of a simple word and Lemma 3.26, if $d \neq 0$.

Consequently $d=0$ and $Z^{2}=P$ in rank $i$. Then $Z$ has finite order in rank $i$ by property (Z1.2). This order must divide $n$ by property (Z1.2), Lemma 3.12 and Lemma 3.26 since $B^{n}=1$ in rank $i$ for every period $B$ of rank $\leq i$. Therefore $Z^{2}=P \in \mathbf{0}(A)$ implies that $Z \in \mathbf{0}(A)$ in rank $i$ as $n$ is an odd number.

By Lemma $3.13 Z$ commutes with $A^{n}$ in rank $i$. It follows that $A^{2 n}=1$ in rank $i$. This is the final contradiction, as simple in rank $i$ word $A$ has infinite order in rank $i$ by Lemma 3.26.

Lemma 3.34. Let $\Delta$ be a g-reduced disc diagram of rank $i$ with the contour $p_{1} q_{1} p_{2} q_{2}$, where $\phi\left(q_{1}\right)$ and $\phi\left(q_{2}\right)$ are periodic words with simple in rank $i$ periods $A$ and $B$. Assume that $\left|p_{1}\right|,\left|p_{2}\right|<\alpha|B|$, $\left|q_{1}\right|>\left(1+\frac{\gamma}{2}\right)|A|,\left|q_{2}\right|>\frac{1}{2} \varepsilon n|B|$. Then the word $A$ is a conjugate in rank $i$ of a word $B^{ \pm 1} R$, where $R \in \mathbf{0}(B)$. Moreover it is equal in rank $i$ to $\phi\left(p_{1}\right)^{-1} B^{ \pm 1} R \phi\left(p_{1}\right)$ if the word $\phi\left(q_{1}\right)$ starts with $A$ and $\phi\left(q_{2}\right)^{-1}$ starts with $B^{-1}$. 
Proof. To prove the lemma, one should repeat the argument from Lemma 19.1 [30] up to the reference to Lemma 3.31, instead of Lemma 18.7 [30] which provides us with the additional factor $R$ as compared with [30].

Lemma 3.35. Let $\Delta$ be a g-reduced disc diagram of rank $i$ with the contour $p_{1} q_{1} p_{2} q_{2}$, where $\phi\left(q_{1}\right)$ and $\phi\left(q_{2}\right)$ are periodic words with simple in rank $i$ periods $A$ and $B ;\left|q_{2}\right| \geq \varepsilon n|B|,\left|p_{1}\right|,\left|p_{2}\right|<\zeta n c$ for $c=\min (|A|,|B|)$. Then either $\left|q_{1}\right|<(1+\gamma)|A|$, or $A$ is a conjugate in rank $i$ of a word $B^{ \pm 1} R$ where $R \in \mathbf{0}(B)$. Furthermore, equality $A \equiv B^{ \pm 1}$ implies $A \equiv B^{-1}$ and the paths $q_{1}, q_{2}$ are $A$-compatible in $\Delta$.

Proof. The statement can be reduced to the claim of Lemma 3.34 in the similar way as in Lemma $19.2[30]$.

Recall that $i_{+}$means 1 if $i=1 / 2$, and $i+1$ if $i \geq 1$.

Lemma 3.36. 1) If $|X| \leq i_{+}$then $X^{n}=1$ in rank $i_{+}$.

2) period $A$ of rank $i_{+}$is simple in any rank $j \leq i$.

3) Let $A$ and $B$ be periods of ranks $k, l \leq i_{+}$, and $A \sim_{j} B$ for some $j<k, l$. Then $A \equiv B$.

Proof. 1) If $X$ is a conjugate of a word of length 0 in rank $i_{+}$, the statement follows from (Z1.2). Otherwise by Lemma $3.25 X$ is a conjugate in rank $i$ of a word of the form $A^{l} P$ with $|A| \leq|X|, P \in$ $\mathbf{0}(A)$ and $A$ is either a period of rank $\leq i$ or a simple word in rank $i$. Then $\left(A^{l} P\right)^{n}=A^{l n}$ by Lemma 3.12. If $|A|<i_{+}$, then $A^{l n}=1$ in rank $i$ by the definition of relators of rank $\leq i$. Hence $X^{n}=1$ in rank $i$, and consequently, in rank $i_{+}$. If $|A|=i_{+}$and so, $A$ is simple in rank $i$, then $A V=Y B^{ \pm 1} R Y^{-1}$ in rank $i$ for a period $B$ of rank $i_{+}$, some $V \in \mathbf{0}(A), R \in \mathbf{0}(B)$ and a word $Y$, by the definition of the set $X_{i_{+}}$. Hence equality $A^{n}=1$ in rank $i_{+}$follows from equality $B^{n}=1$ in rank $i_{+}$and Lemma 3.12.

2) Follows from the inclusion of the set of relators of rank $j$ into the set of relators of rank $i$ for $j \leq i$.

3) If $k$ were less than $l$, for example, then $B=X A^{ \pm 1} P X^{-1}$ in rank $l_{-}$for some $X$ and $P \in \mathbf{0}(A)$ as it was shown at Lemma 3.24 , but this contradicts the choice of periods of rank $l$. If $k=l$, then $A \equiv B$ by the definition of the set $X_{l}$.

Lemmas $3.37-3.39$ will be proved by simultaneous induction on the type of diagrams.

Lemma 3.37. Let $\pi$ be a hub in a g-reduced diagram $\Delta$ of rank $i_{+}$. Then (1) the degree of contiguity of arbitrary cell $\Pi$ to $\pi$ is less than $\varepsilon$; (2)arbitrary contiguity subdiagram $\Gamma$ of $\pi$ to a cell $\Pi$ or to a section $p$ of the boundary $\partial(\Delta)$, labeled by a A-periodic word where $A$ is a simple in rank $i_{+}$word or a period of rank $\leq i_{+}$, is of rank 0 , provided in the last case that there are no cells compatible with $p$, in $\Delta$.

Proof. Consider a contiguity subdiagram $\Gamma$ between a hub $\pi$ and another cell $\Pi$. Let $E$ be one of the bonds defining $\Gamma$. If $r(E)>0$, then the principal cell of $E$ has the contiguity degree to $\pi$ at least $\varepsilon$, and this contradicts the statement of the lemma for a subdiagram of a smaller type (the subdiagram does not contain $\Pi$ ). Thus $E$ is a 0 -bond.

Furthermore the same argument shows that a cell $\pi^{\prime}$ of positive rank from $\Gamma$ possesses no contiguity subdiagram to $\pi$ (to $\Pi$ if $\Pi$ is also a hub), with degree $\geq \varepsilon$. If $\Pi$ is a cell of rank $\geq 1$, 
then by lemmas 3.38, 3.39 for $\Gamma$ and Lemma 3.17, one may apply Lemma 2.3 and conclude that a contiguity degree of $\pi^{\prime}$ to $\Pi$ is less than $\bar{\alpha}$. Since side arcs of $\Gamma$ are of length 0 , this contradicts Lemma 2.4 because $\varepsilon+\bar{\alpha}<\bar{\gamma}$. Thus $r(\Gamma)=0$ if $\pi \neq \Pi$.

If $\Pi$ is a hub, then $(\Pi, \Gamma, \pi)<\varepsilon$ by property (Z2.3) because otherwise a subdiagram containing hubs $\pi$ and $\Pi$ could be replaced by a subdiagram of rank 0 , but $\Delta$ is a g-reduced diagram. If $\Pi$ has rank $\geq 1$, then every maximal $Y$-band of $\Gamma$, starting on $\Pi$, must end on $\pi$ by Lemma 3.2, and so $\Gamma \wedge \Pi$ cannot contain a subword of length $>|A|$ since every letters of $\phi(\Gamma \wedge \pi)$ are distinct by $(Z 2.2)$. Therefore $(\Pi, \Gamma, \pi) \leq \iota<\varepsilon$ by A1, and the lemma claim is proved for a contiguity subdiagram between a hub and another cell.

If $\pi=\Pi$, then again the bond $E$ has rank 0, because otherwise the principal cell $\pi^{\prime}$ of this bond differs from $\pi$, and, by previous case, $\pi^{\prime}$ possesses no contiguity subdiagram of degree $\geq \varepsilon$ to $\pi$ contrary to the definition of a bond. Then $r(\Gamma)=0$ as above, and the assumption $(\pi, \Gamma, \pi)>\varepsilon$ would contradict property (Z2.2).

Finally, if $\Gamma$ is the contiguity subdiagram of the hub $\pi$ to the boundary section $p$ given in the lemma hypothesis, then again the rank of the bond $E$ is 0 as above, and then the same argument shows that $r(\Gamma)=0$.

Lemma 3.38. A g-reduced diagram $\Delta$ of rank $i_{+}$is an A-map.

Proof. 1) Hubs have rank $1 / 2$ and perimeter at least $n$ by property (Z2.1). The perimeter of a cell with a period of a positive integer rank $j \leq i_{+}$is equal to $n j$. Thus $\Delta$ satisfies condition A1.

2) Let $q$ be a subpath of $\partial(\Pi)$, where $r(\Pi)=j \leq i_{+}$, and let $|q| \leq \max (j, 2)$. Let $p$ be a path homotopic to $q$ in $\Delta$, and $\Gamma$ denote the subdiagram with contour $p^{-1} q$. If $\Pi$ does not occur in $\Gamma$, then the type of $\Gamma$ is smaller than that of $\Delta$, and $\Gamma$ is an $A$-map by the inductive hypothesis. If also $|q| \leq 2$ and $|p|<2$, then $|\partial(\Gamma)| \leq 3$, and $r(\Gamma)=0$ by Lemma 2.8. Then $\phi(p)=\phi(q)$ in $\mathbf{G}(0)$, which is impossible by the cyclic irreducibility of periods and, if $\Pi$ is a hub, by (Z2.2).

We suppose next that $|q| \leq j$ and $j>1$. If $|p|<|q|$, then $|\partial(\Gamma)|<2 j$, and by Lemma 2.8 , the perimeters of all cells of positive rank in $\Gamma$ are less than $2 j \bar{\beta}^{-1}$, and so $r(\Gamma)<2 j \bar{\beta}^{-1} n^{-1}<j$ by A1. In this case, one can find a subword $\phi(q)$ of the cyclic permutation of the period $A$ of $\Pi$, equal in rank $j-1$ to the shorter word $\phi(p)$. Thus, one has a simple in rank $j-1$ word $A$ that is a conjugate in rank $j-1$ of a word of length $<|A|$; a contradiction.

We now assume that $\Pi$ occurs in $\Gamma$. Then $\Pi$ does not occur in the subdiagram $\Gamma^{\prime}$ with contour $p q^{\prime}$ where $q^{\prime}$ is the complement of $q$ in the contour of $\Pi$. Thus $\Gamma^{\prime}$ is an $A$-map by the inductive hypothesis. If $r(\Pi) \geq 1$, then, by Lemma 3.172 ), no cell of $\Gamma^{\prime}$ is compatible with $q^{\prime}$. Hence, applying Lemma 3.39 to $\Gamma^{\prime}, q^{\prime}$ is a smooth section in $\partial\left(\Gamma^{\prime}\right)$, and by Lemma $2.7, \bar{\beta}\left|q^{\prime}\right| \leq|p|$. Therefore

$$
|q| \leq 2(n-2)^{-1}\left|q^{\prime}\right| \leq 2 \bar{\beta}^{-1}(n-2)^{-1}|p|<|p| .
$$

It remains to suppose that $\Pi$ is a hub. Then, by Lemma 3.37, there is no cell in $\Gamma^{\prime}$ with a contiguity subdiagram to $q^{\prime}$ of degree $\geq \varepsilon$. Hence $q^{\prime}$ is a smooth section in $\partial\left(\Gamma^{\prime}\right)$. Then again $|q| \leq 2(n-2)^{-1} \bar{\beta}^{-1}|p|<|p|$, which completes the verification of condition A2.

3) Let $\Gamma$ be a contiguity subdiagram of a cell of rank $j$ to a cell of rank $k$ in $\Delta$, with $p_{1} q_{1} p_{2} q_{2}=$ $\partial(\pi, \Gamma, \Pi)$ and $(\pi, \Gamma, \Pi) \geq \varepsilon$. Then $k \geq 1$ by Lemma 3.37 , and $\Gamma$ is an $A$-map by the inductive hypothesis.

Assume first that $j=1 / 2$. Then $r(\Gamma)=0$ by Lemma 3.37. By Lemma 3.2 every maximal $Y$-band of $\Gamma$ starting on $q_{2}$ must end on $q_{1}$, and therefore, by property (Z2.2), all the edges of $q_{2}$ have distinct labels. Hence $\left|q_{2}\right| \leq|A|=k<(1+\gamma) k$ where $A$ is the period of $\Pi$. 
Then suppose that $j \geq 1$. In this case, $q_{1}$ and $q_{2}$ are smooth sections of ranks $j$ and $k$, respectively, in $\partial(\Gamma)$ by Lemma 3.172 ) and Lemma 3.39 applied to $\Gamma$. Thus, applying Lemma 2.1 to $\Gamma$, $\left|p_{1}\right|,\left|p_{2}\right|<\zeta n \min (j, k)$. Hence, by Lemma 2.14, $r=r(\Gamma)<\min (j, k)$, and, by Lemma 3.36, the periods $B, A$ corresponding to $\pi$ and $\Pi$ are simple in rank $r$. Thus, applying Lemma 3.35 to $\Gamma$, either $\left|q_{2}\right|<(1+\gamma)|A|=(1+\gamma) k$ (that is, condition A3 holds), or $A=X B^{ \pm 1} R X^{-1}$ in rank $r$ for $R \in \mathbf{0}(B)$, and therefore $A \equiv B$ by Lemma 3.36. Hence, by Lemma 3.35, $q_{1}$ and $q_{2}$ are compatible, contradicting Lemma 3.17 1). This completes the proof.

Lemma 3.39. Let $p$ be a section of a contour of a g-reduced diagram $\Delta$ of rank $i_{+}$and one of the following two conditions holds.

(1) The path $p$ is not a cyclic section and $\phi(p)$ is an A-periodic word where $A$ is a period of a rank $k \leq i_{+}$, and there are no cells in $\Delta$, compatible with $p$, or a simple word in rank $i_{+}$.

(2)The path $p$ is a cyclic section and $\phi(p) \equiv A^{m} P$ where $A$ is a simple in rank $i_{+}$word and $P \in \mathbf{0}(A)$.

Then $p$ is a smooth section of rank $|A|$ in $\partial(\Delta)$.

Proof. Case 1. Suppose first that assumption (1) from the lemma holds. Let us check two conditions from the definition of a smooth section.

1) Let $q$ be a subpath in $p,|q| \leq \max (|A|, 2)$, and $t$ a path homotopic to $q$ in $\Delta$. The subdiagram $\Gamma$ with contour $q t^{-1}$ is an A-map by Lemma 3.38. If $|t|<|q| \leq 2$, then $|\partial(\Gamma)| \leq 3$, which gives a contradiction as in the proof of Lemma 3.38. We can thus assume that $|q| \leq|A|$. If $A$ is a simple in rank $i_{+}$, word then it is not a conjugate in rank $i_{+}$of a shorter word, and so we have, for the subword $\phi(q)$ of a cyclic permutation of $A,|\phi(q)| \leq|\phi(t)|$, since $\phi(q)=\phi(t)$ in rank $i_{+}$. This enables us to assume that $r(A)=k \leq i_{+}$.

If we now suppose that $|t|<|q|$, then $|\partial(\Gamma)|<2 k$, and by Lemma $2.8, r(\Gamma)=j<k$. Then by Lemma 3.36, $A$ is simple in rank $j$, and we arrive at the previous case. Thus, the first condition in the definition of smoothness of section of rank $|A|$ is verified.

2 ) Let $\Gamma$ be a contiguity subdiagram of a cell $\Pi$ of rank $j$ to $q$, where $\partial(\Pi, \Gamma, q)=p_{1} q_{1} p_{2} q_{2}$ and $(\Pi, \Gamma, q) \geq \varepsilon$. If $j=1 / 2$, then $\left|q_{2}\right| \leq|A|<(1+\gamma)|A|$ as in the proof of Lemma 3.38. Hence we suppose that $j \geq 1$ and $B$ is the period for $\Pi$.

Subdiagram $\Gamma$ is an A-map by Lemma 3.38, and by the inductive hypothesis $q_{2}$ is a smooth section of rank $|A|$ in $\partial(\Gamma)$. The section $q_{1}$ is smooth of rank $j$ by lemmas 3.172$)$ and 3.39 applied to $\Gamma$. Applying Lemma 2.1 to $\Gamma,\left|p_{1}\right|,\left|p_{2}\right|<\zeta n \min (j,|A|)$. Hence, by Lemma $2.14, r=r(\Gamma)<$ $\min (j,|A|)$, and by lemma 3.36, the periods $A$ and $B$ are simple in rank $r$. So, applying Lemma 3.35 to $\Gamma$, either $\left|q_{2}\right|<(1+\gamma)|A|$ (that is, the second condition in the definition of smooth section holds), or $A=X B^{ \pm 1} R X^{-1}$ in rank $r$, where $R \in \mathbf{0}(B)$. Then, by Lemma 3.36, $A \equiv B^{ \pm 1}$, and by Lemma 3.35, $q_{1}$ and $q_{2}$ are $A$-compatible in $\Gamma$, that is, $\Pi$ and $q$ are $A$-compatible in $\Delta$, contrary to the hypothesis of the lemma

Case 2. Suppose now the assumption (2) of the lemma holds. The label of the contiguity arc $q_{2}$ in item 2) (and $\phi(q)$ in item 1)) can be of the form $U_{1} P U_{2}$ where $U_{1} U_{2}$ is a phase decomposition of an $A$-periodic word. By the definition of $\mathbf{0}(A)$, by Lemma 3.6 and by Lemma $3.7 P U_{2}=U_{2} P^{\prime}$ in $\mathbf{G}(0)$, where $P^{\prime} \in \mathbf{0}\left(A^{\prime}\right),\left|P^{\prime}\right|=0, A^{\prime}$ is a cyclic permutation of $A$ and $U \equiv U_{1} U_{2}$ is an $A^{\prime}$-periodic word with phase decomposition $U \cdot 1$. Hence one can transform $\Gamma$ by adding a subdiagram of rank 0 whose contour labelled by $U P^{\prime}\left(U_{1} P U_{2}\right)^{-1}$. The new diagram $\Gamma^{\prime}$ can be considered as in Case 1 , because $\left|P^{\prime}\right|=0$ and $P^{\prime}$ can be can be considered a part of the label of the side $\operatorname{arc}$ of $\Gamma^{\prime}$.

The lemma is proved. 
This together with Lemma 3.36 1) complete the proof of Proposition 3.19.

Proposition 3.19 immediately implies the following statement that shows that one can construct analogs of HNN extensions in the class of groups of odd exponent $n>>1$.

Corollary 3.40. Let $G=\langle A \mid R\rangle$ be a group satisfying the identity $x^{n}=1$ for some odd $n>>1$. Let $P_{i}, Q_{i}$ be subgroups of $G, i=1,2, \ldots, k, k \geq 1$, and for each $i=1, . ., k$, let $\phi_{i}: P_{i} \rightarrow Q_{i}$ be an isomorphism. Let $H$ be the (multiple) HNN extension of $G$ corresponding to these pairs of isomorphic subgroups,

$$
\left.H=\left\langle A, t_{1}, \ldots, t_{k}\right| R, t_{i} u t_{i}^{-1}=\phi_{i}(u), \text { for all } u \in P_{i}, i=1, \ldots, k\right\rangle .
$$

Consider $A$ as a set of 0 -generators and $Y=\left\{t_{1}, \ldots, t_{k}\right\}$ as the set of non-zero generators of $H$. Suppose that $H$ satisfies the conditions (Z3.1) and (Z3.2) (or (Z3.1) and (Z3.2')). Then the natural homomorphism $G \rightarrow H / H^{n}$ is an embedding.

Proof. Indeed, notice that $H$ obviously satisfies conditions (Z1). Conditions (Z2) also are satisfied because there are no hubs. Conditions (Z3) are satisfied by our assumption. Hence by Proposition 3.19 every $g$-reduced diagram over some graded presentation $R \cup S_{1} \cup S_{2} \cup \ldots$ of $H / H^{n}$ is an A-map, and every relator in $S_{i}, i=1,2, \ldots$ is of the form $A^{n}$ where $A$ is a cyclically reduced word of $Y$-length $i$.

Let $W$ be a word in $A$ which is equal to 1 in $H / H^{n}$. Let $\Delta$ be a $g$-reduced van Kampen diagram with boundary label $W$. If the rank of $\Delta$ is at least 1 then by Lemma $2.5 \Delta$ contains a cell $\Pi$ of rank $\geq 1$ and a contiguity subdiagram of rank 0 of $\Pi$ to $\partial(\Delta)$ with non-zero contiguity degree. This implies that the label of $\partial(\Delta)$ must contain a letter from $Y$, a contradiction (we assumed that $W$ contains only letters from $A$ ). Hence $\Delta$ has rank 0 , so $\Delta$ is a diagram over $R$. Hence $W=1$ in $G$.

Remark 3.41. Sergei Ivanov also proved Corollary 3.40[17]. We added this corollary in our paper after the second author attended Ivanov's talk at the Geometric Group Theory conference in Montreal (May, 2002). A particular case of that corollary has been obtained by K.V.Mikhajlovskii [24]. He considered the case when $k=1$, each of the subgroups $P_{1}$ and $Q_{1}$ is malnormal (i.e. $P_{1} \cap x P_{1} x^{-1}=\{1\}$ if $x \in G \backslash P_{1}, Q_{1} \cap x Q_{1} x^{-1}=\{1\}$ if $\left.x \in G \backslash Q_{1}\right)$, and $x P_{1} x^{-1} \cap Q_{1}=\{1\}$ for every $x \in G$. In that case $\mathbf{0}(h)=\{1\}$ for every non-zero cyclically minimal element $h \in H$. Hence condition (Z3) trivially holds.

\section{Subgroups of the free Burnside groups.}

In this section we prove certain properties of free Burnside groups. Although Theorem 4.4 below is of independent interest, this section plays an auxiliary role in the paper and can be skipped in the first reading.

\subsection{Sets of words with small cancellation}

Let $\mathcal{A}=\left\{a_{1}, \ldots, a_{m}\right\}$ be an alphabet. For a constant $c>1$, we say that a word $v$ is $c$-aperiodic if for every word $A$, there are no non-empty $A$-periodic subwords $w$ of $v$ with $|w| \geq c|A|$. 
Lemma 4.1. For every $\varepsilon>0$, one can find $m=m(\varepsilon)$ such that the set of (positive) $(1+\varepsilon)$ aperiodic words in the alphabet $\mathcal{A}_{m}=\left\{a_{1}, \ldots, a_{m}\right\}$ is infinite. Moreover, the number $f(i)$ of such words of length $i \geq 1$ is greater than $m^{(1-\varepsilon / 2) i}$.

Proof. First notice that one can assume that $\varepsilon$ is sufficiently small and fix a number $m$ such that

$$
m-m^{\frac{\varepsilon}{2}+\frac{1}{1+\varepsilon}}-m^{-1+\frac{\varepsilon}{2}+\frac{2}{1+\varepsilon}}>m^{1-\frac{\varepsilon}{2}}-m^{\frac{1}{1+\varepsilon}}
$$

Obviously, $f(1)=m>m^{1-\varepsilon / 2}$. We will prove the inequality $f(i+1)>m^{1-\varepsilon / 2} f(i)$, for $i \geq 1$, by induction on $i$.

By adding a letter to a $(1+\varepsilon)$-aperiodic word $w^{\prime}$ of length $i$ on the right, one obtains $m f(i)$ words of length $i+1$. But some of these words $w$ may contain non-empty $A$-periodic subwords $v$ with $|v| \geq(1+\varepsilon)|A|$ for some $|A|$. We need an upper estimate for the number of such "bad" possibilities.

Clearly, the subword $v$ must be a suffix of $w, w \equiv u v$, since otherwise $v$ would be a subword of the prefix $w^{\prime}$ of length $i$ in $w$. Further we may assume that the word $v$ starts with the period $A$, and the previous argument shows that $A$ is uniquely determined by $v$, because otherwise word $w^{\prime}$ were not $(1+\varepsilon)$-aperiodic. Since $|v| \leq(1+\varepsilon)|A|$, the total number of the words $v$ of length $k$, which can occur, is at most $m^{\frac{k}{1+\varepsilon}}$. Therefore the number of "bad" products $w \equiv u v$ with $|v|=k$ and $|u|=n+1-k$, is at most $f(n+1-k) m^{\frac{k}{1+\varepsilon}}$. By using the inductive hypothesis, one can substitute the first factor by $f(n) m^{(1-k)(1-\varepsilon / 2)}$. Then, by summing over $k \geq 2$, we obtain

$$
\begin{gathered}
f(n+1)>m f(n)-f(n) \sum_{k=2}^{\infty} m^{(1-\varepsilon / 2)(1-k)+\frac{k}{1+\varepsilon}}= \\
f(n)\left(m-\frac{m^{-1+\frac{\varepsilon}{2}+\frac{2}{1+\varepsilon}}}{1-m^{-1+\frac{\varepsilon}{2}+\frac{1}{1+\varepsilon}}}\right)>m^{1-\varepsilon / 2} f(n)
\end{gathered}
$$

by (14), as required.

Lemma 4.2. For any $\varepsilon>0$, there exists $m=m(\varepsilon)$ and an infinite set $S$ of (positive) words $A_{1}, A_{2}, \ldots$ in the alphabet $\mathcal{A}_{m}=\left\{a_{1}, \ldots, a_{m}\right\}$ such that:

(1) every reduced product, whose factors belong to $S$, has no non-empty A-periodic subwords of length $\geq(1+\varepsilon)|A|$ unless the word $A$ is freely conjugate to a product of some words of $S$;

(2) suppose that $A^{\prime} \equiv U V^{\prime}$ and $A^{\prime \prime} \equiv U V^{\prime \prime}$, or $A^{\prime} \equiv V^{\prime} U$ and $A^{\prime \prime} \equiv V^{\prime \prime} U$, are distinct cyclic permutations of the words of $S$ (that is, $U$ is a common prefix or a common suffix). Then $|U|<$ $\frac{\varepsilon}{10} \min \left(\left|A^{\prime}\right|,\left|A^{\prime \prime}\right|\right)$.

(3) $\left|A_{i}\right| \geq n, i=1,2, \ldots$

Proof. We can assume that $\varepsilon<1 / 3$ and choose a number $m_{1}=m_{1}\left(\varepsilon_{1}\right)$ for $\varepsilon_{1}=\varepsilon / 10$ and an infinite set of positive words $B_{1}, B_{2}, \ldots$ in alphabet $\mathcal{A}_{m_{1}}$, according to Lemma 4.1. Then define $m=s m_{1}$ for a number $s \geq 2 \varepsilon_{1}^{-1}$.

Set $A_{i}=B_{i, 1} \ldots B_{i, s}$ for $i=1,2, \ldots$, where $B_{i, j}$ are the copies of the word $B_{i}$ in the alphabet $\mathcal{A}_{j}=\left\{a_{1 j}, \ldots, a_{m_{1} j}\right\}$. Throwing out, if necessary, a finite subset of $\left\{A_{1}, A_{2}, \ldots\right\}$ we can assume that $\left|A_{i}\right| \geq n$. Hence property (3) holds. Property (2) follows from the inequality $s \geq 2 \varepsilon_{1}^{-1}$ because the alphabets $\mathcal{A}_{j}$ are disjoint. 
In particular, in any product $A_{i} A_{j}^{-1}$, we can cancel at most $\varepsilon_{1}$ (in length) of $A_{i}$ or $A_{j}$ if $i \neq j$, and no letter of $B_{i, s-1}$ or $B_{j, s-1}^{-1}$ can be cancelled in the product. Similar remark concerns the product $A_{i}^{-1} A_{j}$.

To prove (1), suppose that $v$ is a non-empty $A$-periodic subword of a reduced form $w \equiv$ $\bar{A}_{i_{1}}^{ \pm 1} \ldots \bar{A}_{i_{l}}^{ \pm 1}$ of a product $A_{i_{1}}^{ \pm 1} \ldots A_{i_{l}}^{ \pm 1}$, and $|v| \geq(1+\varepsilon)|A|$. We may assume that $v \equiv A^{\prime} A^{\prime \prime} A^{\prime}$, where $A \equiv A^{\prime} A^{\prime \prime}$ and $\left|A^{\prime}\right| \geq \varepsilon|A|$, because otherwise we can just shorten the subword $v$.

Assume first that both occurrences $A^{\prime}$ are contained in the same factor $\bar{A}_{i_{r}}^{ \pm 1}$ of $w$. Then $v^{ \pm 1}$ is a subword of the product $B_{i_{r} 1} \ldots B_{i_{r} s}$, and the both occurrences of $A^{\prime}$ are present in some $B_{i_{r} j}$. Hence $v$ is a subword of $B_{i_{r} j}$, contrary to the choice of the words $B_{1}, B_{2} \ldots$

Now assume, the first $A^{\prime}$ of the decomposition of $v$ starts in some $\bar{A}_{i_{l}}^{ \pm 1}$, and the last $A^{\prime}$ ends in some $\bar{A}_{i_{r}}^{ \pm 1}$ with $r>l$. Then the definition of the words $A_{i}$ implies that $v$ must contain at least $\frac{s-3}{2}>\frac{2}{5} s$ factors $B_{i_{l} j}$ of some $A_{i_{k}}$ where $l \leq k \leq r$. Then

$$
\left|A^{\prime}\right| \geq \varepsilon(1+\varepsilon)^{-1}|v|>\varepsilon(1+\varepsilon)^{-1} \frac{2}{5}\left|A_{i_{k}}\right|>3 \varepsilon_{1}\left|A_{i_{k}}\right|
$$

By the choice of $s, A^{\prime}$ contains a subword $\left(B_{i_{k}, t} B_{i_{k}, t+1} B_{i_{k}, t+2} B_{i_{k}, t+3}\right)^{ \pm 1}$ for some $t$. We will suppose that the exponent is equal to +1 . Hence a cyclic permutation of $A$ is freely equal to a word $\left(B_{i_{k}, t+2} B_{i_{k}, t+3}\right) \ldots\left(B_{i_{k}, t} B_{i_{k}, t+1}\right)$. This subword of $w$ must be freely equal to a cyclic permutation of a product of a number of factors $A_{i}^{ \pm 1}$, because there is only one occurrence of the form $B_{j, q} B_{j, q+1}$ in all the words $A_{1}, A_{2}, \ldots$ The lemma is proved.

Remark 4.3. In the proof of Theorem 1.1, we do not need infinite collections of words satisfying the conditions of Lemma 4.2. We only need a collection of words satisfying that conditions and consisting of $C m$ words where $C$ is a constant depending on $n$ (but not on $m$ ), $C>n>\frac{20}{\varepsilon}$, and $m$ is a sufficiently large integer.

An explicit and easy construction of such collections of words can be found in [35]. Let us repeat this construction here. Let us fix integers $r>C^{2}$ and $m=r n$. Consider an $r^{2} \times n$-matrix $M$ where every odd-numbered column is equal to

$$
(1,2, \ldots, r, 1,2, \ldots, r, \ldots, 1,2, \ldots, r)
$$

and every even-numbered column is equal to

$$
(1,1, \ldots, 1,2,2, \ldots, 2, \ldots, r, r, \ldots, r) .
$$

Now let us replace every number $i$ in column $j$ of $M$ by letter $a_{i, j}$. Rows of the resulting matrix can be viewed as words of length $n$ over the alphabet with $m=r n$ letters $\left\{a_{i, j}, i=1, \ldots, r, j=1, \ldots, n\right\}$. For example the first word is equal to $a_{1,1} a_{1,2} \ldots a_{1, n}$, etc. Let $S=\left\{w_{1}, \ldots, w_{r^{2}}\right\}$ be the collection of these words. Let us call words from $S$ and their inverses blocks.

It is mentioned in [35] (and obvious) that no two different words in $S$ have a common subword of length 2. Condition (3) of Lemma 4.2 holds by definition of the blocks. Since $n>\frac{20}{\varepsilon}$, condition (2) of Lemma 4.2 holds.

To prove (1), consider a reduced product $W=w_{i_{1}}^{s_{1}} \ldots w_{i_{k}}^{s_{k}}$ of words from $S, s_{i}= \pm 1$, where $w_{i_{j}}^{s_{j}} w_{i_{j+1}}^{s_{j+1}} \neq 1, j=1, \ldots, k-1$. Suppose that $W$ contains an $A$-periodic subword $V$ where $|V|>$ $(1+\varepsilon)|A|$. Since $n>4 / \varepsilon$, we can assume that $V$ contains a subword $A B$ where $B$ is the beginning of $A$ of length 4 . Then either the first two letters of $B$ are in one block of $W$ or the last two 
letters of $B$ are in one block of $W$. Since every block is completely determined by any of its 2-letter subwords, we can shift $A$ to the left or to the right in $W$, replacing $A$ by a cyclic shift $A^{\prime}$ of $A$, such that $A^{\prime}$ starts with the beginning of a block and ends with the end of a block. Then $A^{\prime}$ is a product of blocks as required.

\subsection{Subgroups of $B(m, n)$ satisfying the congruence extension property}

Recall that a subgroup $H \leq G$ satisfies the congruence extension property if for every normal in $H$ subgroup $N$ there is a normal subgroup $L$ of $G$, such that $N=L \cap H$.

In this section, we prove

Theorem 4.4. For any sufficiently large odd $n$, there exists $m=m(n)$ such that the free Burnside group $B(m, n)$ has a subgroup $H$ with three properties:

(1) $H$ is isomorphic to the free Burnside group $B(\infty, n)$ of the infinite countable rank;

(2) $H$ satisfies the congruence extension property in $B(m, n)$.

(3) the words $A_{1}, A_{2}, \ldots$ representing the free generators of $H$ can be effectively chosen in accordance with Lemma 4.2, and for every $l \geq 1$, the free Burnside subgroup generated by $A_{1}, \ldots, A_{l}$, also possesses the congruence extension property in $B(m, n)$.

The statements (1) and (2) constitute Theorem 1.2. The proof of Theorem 4.4 follows very closely Section 6 of [30] although we repeat a few notation and definitions from [30].

In particular, we use the positive parameters $\alpha, \beta, \gamma, \delta, \varepsilon, \zeta, \iota, n$ from Section 2. Let $m=m(\varepsilon)$ be taken according to Lemma 4.2. Then we fix the infinite set of words $A_{1}, A_{2}, \ldots$ which exists by Lemma 4.2. By Lemma 4.2 (3) the words $A_{k}$ are sufficiently long, say, $\left|A_{k}\right| \geq n$. Let $H$ be the subgroup generated by all words $A_{1}, A_{2}, \ldots$ in the free Burnside group $B(m, n)$ with the free basis $\mathcal{A}=\left\{a_{1}, . ., a_{m}\right\}$.

Suppose that $v$ is a (cyclically) reduced word of length $t$ in certain variables $x_{1}, \ldots, x_{s}$. The (cyclic) word $v\left(A_{1}, \ldots, A_{s}\right) \equiv A_{i_{1}}^{\eta_{1}} \ldots A_{i_{t}}^{\eta_{t}}$ obtained by replacing $A_{i}$ for $x_{i}$ will be called a (cyclic) $H$-word and will be considered together with its decomposition into factors $A_{i_{1}}^{\eta_{1}}, \ldots, A_{i_{t}}^{\eta_{t}}$.

Lemma 4.5. The words $A_{1}, A_{2} \ldots$, satisfying conditions (1) and (2) of Lemma 4.2, freely generate a free Burnside subgroup (of infinite countable rank) of exponent $n$ in the group $B(m, n)$.

Proof. To prove the lemma, we consider the graded presentation of $B(m, n)$ from Section 18 in [30]. Assume there is a non-trivial relation $v\left(A_{1}, \ldots, A_{l}\right)=1$. Then there is a g-reduced diagram $\Delta$ of some rank $i>0$ whose boundary label $\phi(q)$ is freely equal to an $H$-word. Proving by contradiction, we may suppose that the number of cells of $\Delta$ is minimal.

By Lemma 2.5, there is a cell $\Pi$ of $\Delta$ and a contiguity subdiagram $\Gamma$ with $(\Pi, \Gamma, q) \geq \varepsilon$. Since $n \varepsilon>2$, we obtain, by Lemma 4.2(1), (3) that $\Pi$ corresponds to a period $A$ where $A$ is freely conjugate to an $H$-word $\bar{A}$. By Lemma $4.2(2)$ the boundary label $\phi\left(q^{\prime}\right)$ of the subdiagram $\Delta^{\prime}$, obtained from $\Delta$ by cutting off the cell $\Pi$, is freely equal to an $H$-word also, and $\phi(q)$ is freely equal to the product of $\phi\left(q^{\prime}\right)$ and a word $X \bar{A}^{n} X^{-1}$ for some $H$-word $X$. By the minimality of $\Delta$, diagram $\Delta^{\prime}$ corresponds to a relation $v^{\prime}\left(A_{1}, \ldots, A_{l}\right)=1$ such that $v^{\prime}\left(x_{1}, \ldots, x_{l}\right)$ follows from the Burnside identity $x^{n}=1$. Then the relation $v\left(A_{1}, \ldots, A_{l}\right)=1$ follows from Burnside relations too.

Let $\Delta$ be a map. A smooth section $q$ of rank 1 in the contour $\partial(\Delta)$ is said to be $\varepsilon$-section if for any cell $\pi$ of $\Delta$ and any contiguity subdiagram $\Gamma,(\pi, \Gamma, q)<\varepsilon$. 
Let us now fix an arbitrary normal subgroup $N$ in $H$. We would like to define a group presentation of the factor group of $B(m, n)$ by the normal closure of $N$ in $B(m, n)$.

We denote by $T$ the set of all $H$-words (in the alphabet $a_{1}, \ldots, a_{m}$ ) representing elements of $N$, that are cyclically reduced as elements of the free subgroup $H$ (of the absolutely free group) with generators $A_{1}, A_{2}, \ldots$ In particular, $T$ contains all powers of the form $v\left(A_{1}, \ldots, A_{s}\right)^{n}$. The following alteration of the definitions from Section 18 of [30] depends on $N$.

We define $\mathbf{G}(0)=F(\mathcal{A})$ to be the absolutely free group with the empty set $\mathcal{R}_{0}$ of defining words. For every $i \geq 0$ a non-trivial word $A$ is called simple in rank $i$ if it is not conjugate in rank $i$ (i.e. in group $\mathbf{G}(i)$ ) either to a power of a period $B$ of rank $k$, where $1 \leq k \leq i$, or to a power of a word $C$, where $|C|<|A|$, or to an $H$-word.

Every word of $T$ is included in the system $\mathcal{R}_{1}$ of relators of rank 1. (Let us call them $T$-relators.) We also include words $a_{1}^{n}, \ldots, a_{m}^{n}$ in the system $\mathcal{R}_{1}$. Every word $a_{k}$ of length 1 is, by definition, a period of rank 1 , and $\mathbf{G}(1)=\left\langle\mathcal{A} \mid \mathcal{R}_{1}\right\rangle$.

For ranks $i \geq 2$, the definitions of periods of rank $i$ and the group $\mathbf{G}(i)=\left\langle\mathcal{A} \mid \mathcal{R}_{i}\right\rangle, \mathbf{G}(\infty)$, given in Section 18 of [30] remain valid.

Instead of Lemma 18.1 [30] we claim now that the following statement holds.

Lemma 4.6. Every word $X$ over $\mathcal{A}$ is a conjugate in rank $i \geq 0$ either of a power of some period of rank $j \leq i$ or of a power of a simple in rank $i$ word, or of an $H$-word.

The proof can be easily derived from the definition as in [30].

Analogs of Lemma 18.2, Corollaries 18.1 and 18.2 are not used in the proof, so we do not need them now.

The following statement replaces Lemma 18.3 [30].

Lemma 4.7. If a word $X$ has a finite order in rank $i$, then it is a conjugate in rank $i$ of a power of a period of rank $k \leq i$ or of an $H$-word.

There are no changes in the proof as compared with [30].

Assume that $\Pi$ is a $T$-cell (i.e. it corresponds to a $T$-relator) in a g-reduced annular diagram $\Delta$ of rank $i$, and $\Gamma$ is a contiguity diagram of the cell $\Pi$ to itself, such that the hole of $\Delta$ is "surrounded" by $\Pi$ and $\Gamma$, i.e. the union of $\Pi$ and $\Gamma$ does not belong to any disc subdiagram of $\Delta$ (see Figure $12)$.

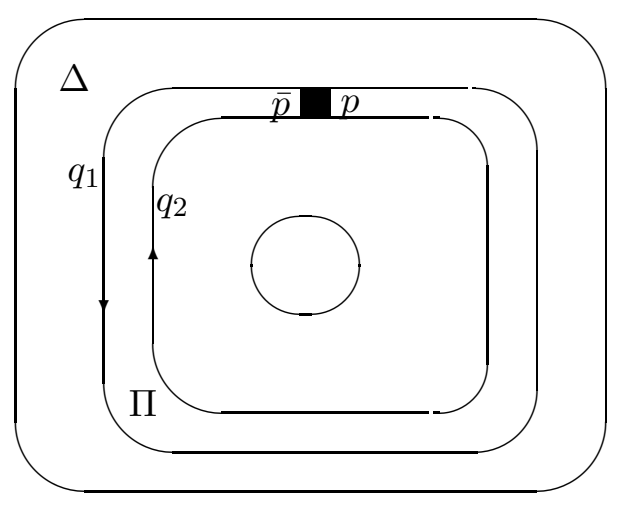

Fig. 12. 
The subdiagram $\Gamma$ is of rank 0 by Lemma 4.13 (1) applied for a smaller rank. If the degree of $\Gamma$-contiguity of $\Pi$ to $\Pi$ is at least $\varepsilon$, we call $\Pi$ a hoop. The reduced boundary $q$ of a hoop can be decomposed as $q_{1} p q_{2} \bar{p}^{-1}$, where $p, \bar{p}$ are the contiguity $\operatorname{arcs}$ of $\Gamma, \phi(\bar{p})$ is freely equal to $\phi(p)$, and so $|p|,|\bar{p}| \geq \varepsilon|q|$. In view of property (2) of Lemma 4.2 , there is a subpath $p^{\prime}$ of $p$, such that $\phi\left(p^{\prime}\right)$ is a unique subword of a unique word $A_{k}^{ \pm 1}$. (More details of such an argument can be found in $\S 2$ of [31].) This implies that the words $\phi\left(q_{1}\right)$ and $\phi\left(q_{2}\right)$ are freely conjugate to some $H$-words. Besides, it follows from $T$-relations that $X \phi\left(q_{1}\right) X^{-1}=\phi\left(q_{2}\right)^{-1}$ for some $H$-word $X$.

Lemma 18.4 in [30] remains unchanged:

Lemma 4.8. If $A$ and $B$ are simple in rank $i$ and $A$ is equal in rank $i$ to $X B^{l} X^{-1}$ for some $X$ then $l= \pm 1$.

The proof does not change much also. Notice only that by definition, a simple word of rank $i \geq 1$ cannot be conjugate in rank $i$ of an $H$-word. Therefore the diagram $\Delta$ from the proof of Lemma 18.4 [30], cannot have hoops, and so the analog of Lemma 19.4 [30] (see Lemma 4.15 below) is applicable.

The formulation of Lemma 18.5 changes as follows.

Lemma 4.9. If words $X$ and $Y$ are conjugate in rank $i$ and $X$ is not a conjugate of any $H$-word in rank $i$ then there exists a word $Z$ such that $X$ is equal to $Z Y Z^{-1}$ in rank $i$ and $|Z| \leq \bar{\alpha}(|X|+|Y|)$.

The additional assumption that $X$ is not a conjugate of an $H$-word again allows us to apply Lemma 4.15, the analog of Lemma 19.4 [30].

Consider a section $q$ of a contour of some diagram $\Delta$, where $\phi(q)$ is freely equal to an $H$-word. We call $q$ an $H$-section. Assume that there is a $T$-cell $\Pi$ in $\Delta$ with boundary path $p$ starting with a vertex $o$ of $\partial(\Pi)$, and there is a path $t$ having no self-intersections, which connects $o$ and a vertex $o^{\prime}$ cutting $q$ into a product $q_{1} q_{2}$. We say that $\Pi$ is compatible with $q$, if the boundary label of the path $q_{1} t^{-1} p t q_{2}$, without self-intersections, is freely equal to an $H$-word. Obviously, one can cut such a cell out of $\Delta$ replacing $q$ by another $H$-section. The definition of compatibility can be generalized in the case when $\phi(q)$ is a subword of a reduced form $W$ of an $H$-word with $|\phi(q)| \geq \varepsilon|W|$ in view of Lemma 4.2(2). (Such a path $q$ is also an $H$-section by definition.)

Similarly, one defines the compatibility of two $T$-cells in a diagram. A pair of two compatible $T$-cells can be substituted by (at most) one $T$-cell. Therefore a g-reduced diagram of some rank $i$ cannot contain pairs of compatible $T$-cells or $j$-pairs in the terminology of [30] for $j=1$. For Burnside cells (i.e. cells corresponding to relations of the form $A^{n}=1$ where $A$ is a period of rank $j>0)$ we are using the same definition of $j$-pairs as in [30].

Lemma 4.10. Assume that a word $W$ is a conjugate of an $H$-word in rank $i$. Then $W=U V U^{-1}$, in rank $i$, for some $H$-word $V$ and some word $U$, such that $|V| \leq \bar{\beta}^{-1}|W|$ and $|U| \leq 2|W|$.

Proof. Consider a g-reduced annular diagram $\Delta$ for the conjugacy of $W$ to some $H$-word $V$. We may assume that there are no hoops in $\Delta$, because otherwise one can replace $\Delta$ by a diagram of a smaller type, that also demonstrates the conjugacy of $W$ to a subword of the boundary label of a hoop. For the same reason, we may assume that $\Delta$ has no $T$-cells compatible with the contour, say $p$, of $\Delta$ labeled by $V$. Then $\Delta$ is an $A$-map by Lemma 4.15, and $p$ is a smooth section of $\partial(\Delta)$ 
by Lemma 4.16. Then $|V| \leq \bar{\beta}^{-1}|W|$ by Lemma 2.7 , and the word $U$ can be taken in such a way that

$$
|U| \leq \bar{\alpha}\left(1+\bar{\beta}^{-1}\right)|W| \leq 2|W|
$$

by Lemma 4.9 .

The following Lemma implies malnormality of the subgroup $H$ in $B(m, n)$.

Lemma 4.11. Let $V$ and $W$ be two non-trivial in rank $i H$-words and $W=U V U^{-1}$ in rank $i$. Then the word $U$ is itself equal in rank $i$ to an $H$-word.

Proof. Let $\Delta$ be a g-reduced annular diagram of rank $i$ with contours $p$ and $q$ labeled by $V$ and $W$ respectively. One can assume that there is a path $t=q_{-}-p_{-}$in $\Delta$, such that $\phi(t)=U$ in rank $i$. Then it suffices to show that $\phi(t)$ is freely equal to an $H$-word.

One can suppose that $\Delta$ has no $T$-cells compatible with the contours, because otherwise our problem reduces to a similar problem for an annular diagram of smaller type. If $\Delta$ has a hoop, then $t$ can be constructed as a product $t_{1} t_{2} t_{3}$ where the path $t_{2}$ cuts the hoop and $t_{1}, t_{3}$ are the cutting paths for annular subdiagrams of smaller types whose labels are $H$-words by induction. Thus, we may assume that there are no hoops in $\Delta$. Then both $p$ and $q$ are $\varepsilon$-sections by Lemma 4.14. It follows that $\Delta$ has rank 0 , because otherwise the inequality $\bar{\gamma}>2 \varepsilon$, Lemma 2.4 and Lemma 4.15 lead to a contradiction. Then the desired property of $\Delta$ follows from the malnormality of the subgroup generated by the words $A_{1}, A_{2}, \ldots$ in the absolutely free group $F(\mathcal{A})$, which, in turn, can be easily derived from the statement of Lemma $4.2(2)$.

Next as in [30, Section 18] we prove analogs of lemmas $18.6,18.7,18.8$ by a simultaneous induction on the sum $L$ of the two periods involved in the formulations of these lemmas. The formulations of these lemmas do not change.

Lemma 4.12. (Analog of Lemma 18.6 [30].) Let $\Delta$ be a g-reduced disc diagram of rank $i$ with contour $p_{1} q_{1} p_{2} q_{2}$ where $\phi\left(q_{1}\right)$ and $\phi\left(q_{2}^{-1}\right)$ are periodic words with period $A$ simple in rank $i$. If $\left|p_{1}\right|,\left|p_{2}\right|<\alpha|A|$ and $\left|q_{1}\right|,\left|q_{2}\right|>\left(\frac{5}{6} h+1\right)|A|$, then $q_{1}$ and $q_{2}$ are $A$-compatible in $\Delta$. (The inductive parameter is $L=|A|+|A|$.)

Proof. As in the proof of Lemma 18.6 from [30], assume that $A$ is a prefix of both $\phi\left(q_{1}\right)$ and $\phi\left(q_{2}^{-1}\right)$ by making if necessary $p_{1}$ a little longer (by at most $1 / 2|A|$ ). The new path is also denoted by $p_{1}$.

Notice first that if $\phi\left(p_{1}\right)$ is not a conjugate of an $H$-word in rank $i$ then in any g-reduced annular diagrams of rank $\leq i$ where one of the contours is labeled by $\phi\left(p_{1}\right)$, there are no hoops. So the proof proceeds as in [30].

Otherwise, as in [30], consider the annular diagram $\bar{\Delta}$ of rank $i$ with boundary labels $W \equiv \phi\left(p_{1}\right)$ and $W^{\prime} \equiv \phi\left(q^{\prime}\right) \phi\left(p_{2}\right)^{-1}$. Then $W, W^{\prime}$ are conjugate in rank $i$ of some $H$-word. If $W=1$ in rank $i$ then there is nothing to prove. Thus we assume that the word $W$ is non-trivial in rank $i$.

Inequalities (2), (3) from Section 18 of [30] are still valid:

$$
|W|<\bar{\alpha}|A|, \quad\left|W^{\prime}\right|<\left(\bar{\beta}^{-1}-1\right)\left(\frac{5}{6} \delta^{-1}|A|+|A|\right)+2|A|<\left(\bar{\beta}^{-1}-1\right)\left(\left|q_{2}\right|+2|A|\right)
$$

By Lemma 2.10, one can find $H$-words $V$ and $V^{\prime}$ such that

$$
|V| \leq \bar{\beta}^{-1}|W|<\bar{\beta}^{-1} \bar{\alpha}|A|<|A|, \quad\left|V^{\prime}\right|<\bar{\beta}^{-1}\left(\bar{\beta}^{-1}-1\right)\left(5 / 6 \delta^{-1}|A|+|A|\right)<\beta \delta^{-1}|A|,
$$


and, in rank $i, W=U V U^{-1}, W^{\prime}=U^{\prime} V^{\prime} U^{\prime-1}$, where $|U|<2|A|,\left|U^{\prime}\right|<2 \beta \delta^{-1}|A|$ by Lemma 4.10.

On the other hand, $Q W Q^{-1}=W^{\prime}$, in $\operatorname{rank} i$, for $Q \equiv \phi\left(q_{2}\right),|Q|>\frac{5}{6} \delta^{-1}|A|\left(\right.$ recall that $\left.h=\delta^{-1}\right)$. Therefore $H$-words $V$ and $V^{\prime}$ are conjugate in rank $i$ by the product $U^{\prime-1} Q U$. By Lemma 4.11, this product must be equal, in rank $i$ to an $H$-word $X$.

Let $\Delta_{0}$ be a g-reduced diagram of rank $i$ for the equality $\left(U^{\prime}\right)^{-1} Q U=X$. Its contour has a natural decomposition $\left(u^{\prime}\right)^{-1} q u x^{-1}$. By changing $H$-word $X$, one can suppose that there are no $T$-cells compatible with $x$ in $\Delta$. Hence this section is an $\varepsilon$-section of $\Delta_{0}$ by Lemma 4.14 . Therefore the sum of the degrees of contiguity of an arbitrary cell $\Pi$ of $\Delta$ to $q$ and to $x$, is less than $\bar{\alpha}+\varepsilon<2 / 3$ by Lemmas 2.3 and lemma 4.16 below. Recall that $Q$ is a periodic word with the simple in rank $i$ period $A$.

Notice that

$$
\left|u^{\prime}\right|+|u|<2\left(\beta \delta^{-1}+1\right)|A|<\frac{5}{6} \alpha^{2} \delta^{-1}|A|<\alpha^{2}|q| .
$$

Thus, there is an 0-bond between $q$ and $x$ by Lemma 2.10 (we have mentioned above that the other possibility from that lemma is not possible). Then $q=q(1) e q(2)$, for the contiguity edge $e$ of the 0-bond. If the length of $q(1)$ or $q(2)$ is greater than $2 \alpha^{-2}\left(\beta \delta^{-1}+1\right)|A|$, then one is able to get a new 0-bond between $q$ and $x$ by Lemma 2.10. By keeping obtaining new and new such 0-bonds, we conclude, that there is a subpath $q(0)$ of $q$ and a contiguity subdiagram $\Gamma(0)$ of rank 0 between $q(0)$ and $x$, where $|q(0)|>|q| / 2>2|A|>(1+\varepsilon)|A|$.

Consequently, a reduced form of the $H$-word $X^{-1}$ has an $A$-periodic subword of length $>$ $(1+\varepsilon)|A|$. By Lemma 4.2(1), the word $A$ is itself freely conjugate to an $H$-word. This contradicts the definition of simple words and completes the proof of our lemma.

The proofs of Lemmas 18.7, 18.8 remain unchanged.

The formulations of lemmas 18.9 - 19.3 [30] are left unchanged. The proofs are also unchanged. Only when we apply our analog of Lemma 18.3 (Lemma 4.7 above) in the proof of Lemma 18.9, we take into account that by $T$-relations every $H$-word has order dividing the odd number $n$ in rank $i \geq 1$. In the proof of the first statement of Lemma 19.3, one should remember that the reduced form of $W^{n}$ is included in the list of $T$-relators for an arbitrary $H$-word $W$.

In order to prove analogs of Lemmas 19.4 and 19.5 [30], we need two more Lemmas 4.13 and 4.14. These two lemmas and our analogs of Lemmas 19.4, 19.5 [30] will be proved by a simultaneous induction on the number of positive cells.

Lemma 4.13. Let $\Gamma$ be a contiguity subdiagram of a cell $\pi$ to a $T$-cell $\Pi$ in a g-reduced diagram $\Delta$ of rank $i+1$. Then:

(1) the $\operatorname{rank} r(\Gamma)$ is 0 and

(2) if $\pi \neq \Pi$, then $(\pi, \Gamma, \Pi)<\varepsilon$.

Proof. We can assume that the statement of the lemma is not true and the triple $(\pi, \Gamma, \Pi)$ is a minimal counterexample in the sense that $|\Gamma(2)|$ is minimal possible.

Since $|\Gamma(2)|<|\Delta(2)|$, the subdiagram $\Gamma$ is an $A$-map by Lemma 4.15. Let $p_{1} q_{1} p_{2} q_{2}=\partial(\pi, \Gamma, \Pi)$. Notice that $\left|p_{1}\right|=\left|p_{2}\right|=0$, because otherwise the principal cell of one of the bonds defining $\Gamma$ would be a positive cell with degree of contiguity to $\Pi$ at least $\varepsilon$, which would contradict the minimality of our counterexample.

There are no cells in $\Gamma$ which are compatible with either $q_{1}$ or $q_{2}$ because otherwise these cells would be compatible with cells in $\Delta$, and $\Delta$ would be a non-g-reduced diagram. Then $q_{2}$ is an 
$\varepsilon$-section by Lemma 4.14(2) for $\Gamma$. Also $q_{1}$ is a smooth section in $\partial(\Gamma)$ by Lemma 4.16 or $4.14(2)$. Therefore the sum of contiguity degrees of any cell of $\Gamma$ to $q_{1}$ and $q_{2}$ is less than $\bar{\alpha}+\varepsilon<\bar{\gamma}$, and so $r(\Gamma)=0$ by Lemma 2.4 .

Assume that $(\pi, \Gamma, \Pi) \geq \varepsilon$. It follows from Lemma 4.2(2) that the cells $\pi$ an $\Pi$ are compatible if $\pi$ is a $T$-cell. But this is impossible in the g-reduced diagram $\Delta$. If $\pi$ is not a $T$-cell, we obtain a contradiction to the definition of periods by Lemma 4.2(1). Thus the lemma is proved by contradiction.

Lemma 4.14. Let $\Gamma$ be a contiguity diagram of a cell $\pi$ to an $H$-section $q$ of the boundary of a $g$-reduced diagram $\Delta$ of rank $i+1$. Assume there are no $T$-cells compatible with $q$ in $\Delta$. Then:

(1) $r(\Gamma)=0$ and

(2) $q$ is an $\varepsilon$-section of $\partial(\Delta)$.

Proof. The proof is analogous to the proof of Lemma 4.13.

The formulation of Lemma 19.4 of [30] is modified:

Lemma 4.15. A g-reduced diagram of rank $i+1$, having no hoops, is an A-map.

Proof. The perimeter of any $T$-cell is at least $n$ by Lemma 4.2 (3), and so condition A1 is true. The proof of part A2 remains the same except at the end the argument related to $A$-compatibility should be replaced by a similar argument related to the compatibility of a $T$-cell with an $H$-section, if the cell $\Pi$ is a $T$-cell. The assumption $(\pi, \Gamma, \Pi) \geq \varepsilon$, in part A3 means that $\Pi$ is not a $T$-cell by Lemma 4.13(2), since $\pi \neq \Pi$ (the diagram contains no hoops). Therefore there are no changes in the proof of this part if $\pi$ is not a $T$-cell as well. Otherwise the inequality $\left|q_{2}\right|<(1+\gamma)|A|$ follows from Lemma 4.13(1) and Lemma 4.2(1) (because $\gamma>\varepsilon$ ).

We add one more possibility to the hypothesis of the analog of Lemma 19.5 in [30]. This lemma is now formulated as follows.

Lemma 4.16. Let $p$ be a section of the contour of a g-reduced diagram $\Delta$ of rank $i+1$ and one of the following possibilities holds:

- The label of $p$ is an A-periodic word where $A$ is simple in rank $i+1$,

- The label of $p$ is an A-periodic word where $A$ is a period of rank $k \leq i+1$, and $\Delta$ has no cells of rank $k$ A-compatible with $p$.

- The label of $p$ is an $H$-word and there are no T-cells in $\Delta$ which are compatible with $p$.

(If $p$ is a cyclic section then in the first two cases we further require that $\phi(p) \equiv A^{m}$ for some integer $m$.) Then $p$ is a smooth section of rank $|A|$ in the first and the second cases and of rank 1 in the third case, in $\partial(\Delta)$.

Proof. We need to prove the two properties from the definition of a smooth section.

1. The proof that every subpath of $p$ of length $\leq \max (k, 2)$ is geodesic in $\Delta$ coincides with part 1 ) in the proof of Lemma 19.5 [30] if $\phi(p)$ is not an $H$-word. In the case when $\phi(p)$ is an $H$-word, this property follows from Lemmas 4.13, 2.8 and 4.2 .

2. The proof that for every contiguity submap $\Gamma$ of a cell $\pi$ to $p$ satisfying $(\pi, \Gamma, p) \geq \varepsilon$ we have $|\Gamma \wedge p|<(1+\gamma)(i+1)$ proceeds along the lines of part 2) of Lemma 19.5 [30]. But we need to 
consider a new case when $\pi$ is a $T$-cell. In this case, $r(\Gamma)=0$ as in the proof of Lemma 4.13 (one should just replace $\Pi$ by $p)$. Then the inequality $\left|q_{2}\right| \leq(1+\gamma)(i+1)$ follows from Lemma 4.2(1) and from the definition of $T$-relations.

If $\phi(p)$ is an $H$-word, then the second property of smooth sections follows from Lemma 4.14 $(2)$.

Lemma 4.17. The constructed group $\mathbf{G}(\infty)$ satisfies identity $x^{n}=1$.

Proof. This immediately follows from Lemma 19.3 (1) [30] (its formulation remains the same in our situation). That lemma states that the order of every word of length at most $i$, in rank $i$, is a divisor of $n$.

Proof of Theorem 4.4. By Lemma 4.5, it remains to show that for arbitrary normal in $H$ subgroup $N$, the intersection of the normal closure $L$ of $N$ in $B(m, n)$ and $H$ is equal to $N$, i.e. any $H$-word $w$ representing an element of $L$, represents an element of $N$.

Consider the group $\mathbf{G}(\infty)$ constructed above for this particular $N$. Since $w$ represents an element from $L$ in $B(m, n)$, it belongs to the normal closure, in the absolutely free group, of set $T$ plus the set of all words of the form $A^{n}=1$. By Lemma 4.17 and the definition of $\mathbf{G}(1)$ then $w$ represents 1 in $\mathbf{G}(\infty)$. Therefore there is a g-reduced diagram $\Delta$ of some rank $i$ whose boundary label $\phi(q)$ is freely equal to $w$.

By contradiction assume that the $H$-word $w$ does not represent an element of $N$, and the diagram $\Delta$ has minimal possible number of positive cells.

By Lemma 2.5, there are a cell $\Pi$ of $\Delta$ and a contiguity subdiagram $\Gamma$ of rank 0 with $(\Pi, \Gamma, q) \geq \varepsilon$. If $\Pi$ is a $T$-cell, then it must be compatible with $q$ by Lemma 4.2(2). Therefore when we cut the cell $\Pi$ from $\Delta$, we multiply (in the free group) the boundary label of $\Delta$ by a word $U$ of the form $V W V^{-1}$ where $W$ is the boundary label of $\Pi$, and $V$ is an $H$-word. Since $W$ represents an element of $N, V$ represents an element of $H$, and $N$ is normal in $H$, the word $U$ represents an element of $N$. Hence the boundary label of the resulting diagram $\Delta_{1}$ is an $H$-word which does not represent an element from $N$. So $\Delta_{1}$ is a counterexample with a smaller number of positive cells. If $\Pi$ is not a $T$-cell, i.e. it corresponds to a relation $A^{n}=1$, then we get a contradiction with Lemma 4.14(2).

It is clear that if $H$ is a subgroup of $B(m, n)$ satisfying properties (1) and (2) from Theorem 4.4 then any subgroup generated by finitely many of the free generators of $H$ is also free in the variety of Burnside groups of exponent $n$, and has the congruence extension property because retracts of subgroups with the congruence extension property also have this property and because the congruence extension property is transitive (if $H_{1}$ has the congruence extension property in $H$ and $H$ has it in $\mathbf{G}$ then $H_{1}$ has the congruence extension property in $\mathbf{G}$ ).

Theorem 4.4 (and Theorem 1.2) are proved.

\subsection{Burnside groups and free products}

The following lemma is Theorem VI.3.2 from [2] (or Theorem 19.5 from [30]).

Lemma 4.18. The centralizer of every non-trivial element of $B(m, n)$ is cyclic of order $n$.

We are going to use the following Lemma repeatedly in Section 6.

Lemma 4.19. If $a, b, c \in B(m, n), n>>1$ odd, $a \neq 1, c \neq 1,[a, c]=1,\left[b a b^{-1}, c\right]=1$ then $[b, c]=[b, a]=1$. 
Proof. Indeed, suppose that $[a, c]=1,\left[b a b^{-1}, c\right]=1$. By Lemma 4.18, the centralizer of every non-trivial element of $B(m, n)$ is cyclic of order $n$. Hence $a, b a b^{-1}, c$ belong to the same cyclic subgroup $C$ of $B(m, n)$. Therefore $b C b^{-1}$ and $C$ are non-trivially intersecting cyclic subgroups of order $n$. By Lemma 4.18, $b C b^{-1}=C$. Hence $\langle b, C\rangle$ is a finite subgroup of $B(m, n)$ (or order at most $\left.n^{2}\right)$. By Theorem 19.6 from [30], $\langle b, C\rangle$ is cyclic of order $n$, so $b \in C$. Hence $[b, c]=1$ as required.

In order to prove Proposition 6.17 below, we will need some properties of free products. Let $P$ and $Q$ be two free groups. Elements of the free product $P * Q$ are represented by words of the form $p_{1} q_{1} p_{2} \ldots q_{s} p_{s+1}$ where $p_{i} \in P, i=1, \ldots, s+1$, is called a $P$-syllable, $q_{i} \in Q, i=1, \ldots, s$, is called a $Q$-syllable. By definition, a $P$-syllable (resp. $Q$-syllable) is a maximal subword over the set of generators of $P$ (resp. $Q$ ). Elements of $P$ and $Q$ are called 1-syllable elements.

A word $p_{1} q_{1} \ldots p_{s} q_{s} p_{s+1}$ from $P * Q$, where $p_{i}, q_{j}$ are syllables, is called ${ }^{*}$-reduced in $P * Q$ if none of its syllables except possibly for $p_{1}, p_{s+1}$ is equal to 1 . If a product is *-reduced, it is not equal in $P * Q$ to such a product with smaller number of syllables. The $P$-syllable $p_{s+1}$ is called the last syllable of $p_{1} q_{1} \ldots p_{s} q_{s} p_{s+1}$ (even if $p_{s+1}$ is empty). If in $p_{1} q_{1} \ldots p_{s} q_{s} p_{s+1}$, a syllable, say, $p_{i}$, is freely equal to 1 , we can remove it and glue $q_{i-1}$ with $q_{i}$ to form a new $Q$-syllable. Applying this operation several times, we get a ${ }^{*}$-reduced in $P * Q$ form of the product which is unique. Notice that a word which is $*$-reduced in $P * Q$ may not be freely reduced (as a word written in the free generators of $P$ and $Q$ ).

Let $\psi: P \rightarrow \bar{P}$ be a homomorphism of $P$ onto a group $\bar{P}$ satisfying the identity $x^{n}=1$. We say that a word $h$ of $P * Q$ is $\psi$-trivial if the $\psi$-image of every syllable of $h$ is 1 in $\bar{P}$.

Lemma 4.20. Let $h_{1}, h_{2}$ be words in $P * Q$ which are equal modulo Burnside relations. Then the following properties hold.

(i) If $h_{1}$ is $\psi$-trivial, and $h_{2}$ is of minimal length among all words which are equal to $h_{1}$ modulo Burnside relations then $h_{2}$ is $\psi$-trivial.

(ii) Suppose that $h_{1}$ has exactly two P-syllables $p, p^{\prime}$ with non-trivial $\psi$-images, $h_{1} \equiv x p y p^{\prime} z$, and $y$ is not equal to a word in $P$ modulo Burnside relations. Then $h_{2}$ is not $\psi$-trivial.

(iii) If $h_{1}$ is $\psi$-trivial, $h_{1} \neq 1$ modulo Burnside relations, $h_{2} \in P$, and modulo Burnside relations $h_{1}=x h_{2} x^{-1}$ for some $x \in P * Q$, then there exists a word $x_{1} \in P * Q$ such that $x_{1} h_{2} x_{1}^{-1}=h_{1}$ modulo Burnside relations and the word $x_{1} h_{2} x_{1}^{-1}$ is $\psi$-trivial.

Proof. (i) Obviously we can assume that $h_{1}$ is ${ }^{*}$-reduced. Consider a g-reduced diagram $\Delta$ over the graded presentation of the free Burnside quotient of $P * Q$ from Section 18 of [30] corresponding to the equality $h_{1}=h_{2}$. If the rank of $\Delta$ is 0 then $h_{1}=h_{2}$ in $P * Q$, and each syllable of the word $h_{2}$ (of minimal length) must be a product of several syllables of $h_{1}$. Hence $h_{2}$ is $\psi$-trivial.

Suppose that the rank of $\Delta$ is $>0$, and $\partial(\Delta)=s_{1} s_{2}$ where $\phi\left(s_{1}\right)=h_{1}, \phi\left(s_{2}^{-1}\right)=h_{2}$. Since $s_{2}$ is geodesic, $s_{2}$ is a smooth section of rank $\left|s_{2}\right|$. By Lemma 2.4, part a), and Lemma 2.3 there exists a cell $\Pi$ of rank $>0$ and its contiguity subdiagram $\Gamma$ to $s_{1}$ of contiguity degree $>1 / 2-\gamma-\alpha>3 \varepsilon$. By Lemma 2.6, $\Delta$ contains a cell $\pi$ and a rank 0 contiguity subdiagram $\Gamma^{\prime}$ of $\pi$ to $s_{1}$, such that the contiguity degree of $\Gamma^{\prime}$ is $>\varepsilon$. Let $t$ be the contiguity arc of $\Gamma^{\prime}$ on $\partial(\pi),|t|>\varepsilon|\partial(\pi)|$. There are two possibilities.

Case 1. The period of $\phi(\partial(\pi))$ is a 1-syllable word. Then $\phi(\partial(\pi))$ is a 1-syllable word, and one can cut the subdiagram $\pi \cup \Gamma^{\prime}$ from $\Delta$ preserving the $\psi$-triviality of $\phi\left(s_{1}\right)$. Here we use the fact that $\bar{P}$ satisfies the identity $x^{n}=1$. 
Case 2. The period $A$ of $\phi(\partial(\pi))$ contains at least 2 syllables. Since $\Gamma^{\prime}$ is a diagram of rank 0 , and $\varepsilon n>2$, every syllable of $A$ is a syllable of $\phi\left(s_{1}\right)^{ \pm 1}=h_{1}^{ \pm 1}$. Hence $A$ is $\psi$-trivial (since $h_{1}$ is $\psi$-trivial). Therefore, again, one can cut the subdiagram $\Gamma^{\prime} \cup \pi$ from $\Delta$ preserving the $\psi$-triviality of $\phi\left(s_{1}\right)$ : we replace subword $A$ of $\phi\left(s_{1}\right)^{ \pm 1}$ by $A^{1-n}$ and reduce the resulting word.

Thus in each of these cases we replace $\Delta$ by a diagram of smaller type, and complete the proof by induction on the type of $\Delta$.

(ii) By contradiction assume that $h_{2}$ is $\psi$-trivial. We have that $p y p^{\prime}=x^{-1} h_{2} z^{-1}$. By part (i) of this lemma we can replace $x^{-1} h_{2} z^{-1}$ by a $\psi$-trivial word $h$ of minimal possible length. Consider a g-reduced diagram $\Delta$ with $\partial(\Delta)=s_{1} t_{1} s_{2} t_{2}$ where $\phi\left(s_{1}\right)=p, \phi\left(t_{1}\right)=y, \phi\left(s_{2}\right)=p^{\prime}, \phi\left(t_{2}^{-1}\right)=h$. If the rank of $\Delta$ is 0 then $p y p^{\prime}=h$ in $P * Q$. Since $y$ is not equal to a word from $P$ and is $\psi$-trivial, and $\psi(p) \neq 1$, we have that the ${ }^{*}$-reduced in $P * Q$ form of $p y p^{\prime}$ is not $\psi$-trivial, a contradiction with the $\psi$-triviality of $h$.

If the rank of $\Delta$ is $>0$ then as in part (i), we get a cell $\pi$ and a contiguity subdiagram $\Gamma^{\prime}$ of $\pi$ to one of the subpaths $s_{1}, t_{1}, s_{2}$ with contiguity degree $\geq \varepsilon$, such that $\Gamma^{\prime}$ has rank 0 . Since $\phi\left(s_{1}\right)$ and $\phi\left(s_{2}\right)$ are 1-syllable words, the period $A$ of $\pi$ is a 1-syllable word if $\Gamma^{\prime}$ is a contiguity subdiagram to $s_{1}$ or $s_{2}$. If $\Gamma^{\prime}$ is a contiguity subdiagram to $t_{1}, A$ is a $\psi$-trivial word. As in part (i) we can remove $\Gamma^{\prime} \cup \pi$ from $\Delta$ preserving the structure of the boundary of $\Delta$ and the condition that $\phi(y)$ is a $\psi$-trivial, and does not belong to $P$ modulo Burnside relations, $\phi\left(s_{1}\right), \phi\left(s_{2}\right)$ are 1-syllable words from $P, \psi\left(\phi\left(s_{1}\right)\right), \psi\left(\phi\left(s_{2}\right)\right) \neq 1$. We can complete the proof by induction of the type of $\Delta$.

(iii) Consider a g-reduced annular diagram $\Delta$ with pointed contours $s_{1}, s_{2}$, where $\phi\left(s_{1}\right) \equiv$ $h_{1}, \phi\left(s_{2}\right) \equiv h_{2}$. If the rank of $\Delta$ is 0 then $h_{1}=x h_{2} x^{-1}$ in $P * Q$ and $x h_{2} x^{-1}$ is $\psi$-trivial.

Suppose that the rank of $\Delta$ is $>0$. As in parts (i), (ii), we can remove a cell of rank $>0$ from $\Delta$ and change $s_{1}$ or $s_{2}$ preserving $\phi\left(s_{1}\right), \phi\left(s_{2}\right)$ modulo Burnside relations. The labels of the contours $s_{1}^{\prime}, s_{2}^{\prime}$ of the new annular diagram satisfy all the conditions of part (iii) of the lemma, and again we can complete the proof by induction on the type of $\Delta$.

Lemma 4.21. Suppose that $h, g \in P * Q$, and there is no $x \in P * Q$ such that $g=x p x^{-1}, h=x p^{\prime} x^{-1}$ modulo Burnside relations for some $p, p^{\prime} \in P$. Suppose also that $h$, the ${ }^{*}$-reduced forms of $g g^{-1}$, and $a$ word $b$ which is equal to $g^{-1}$ hg modulo Burnside relations are $\psi$-trivial. Then $g$ is $\psi$-trivial.

Proof. Suppose that $g$ is not $\psi$-trivial. Let $g \equiv g_{1} p g_{2}$ where $g_{2}$ is $\psi$-trivial, $p$ is a $P$-syllable, $\psi(p) \neq 1$. Consider the ${ }^{*}$-reduced form of $g h g^{-1} \equiv g_{1} p g_{2} h g_{2}^{-1} p^{-1} g_{1}^{-1}$. Since this word is $\psi$-trivial, and $g_{2}$ is also $\psi$-trivial, the syllable $p$ is glued with a $P$-syllable $p_{h}$ of $h$ and with the $P$-syllable $p^{-1}$ of $g^{-1}$. This means that $h=g_{2}^{-1} p_{h} g_{2}$ in the free group $P * Q$, and the ${ }^{*}$-reduced form of $g h g^{-1}$ is $g_{1} p p_{h} p^{-1} g_{1}^{-1}$. This implies that $g_{1}$ is $\psi$-trivial, so $g$ has only one $P$-syllable with non-trivial $\psi$-image. Now consider the ${ }^{*}$-reduced form $b^{\prime}$ of the word $g^{-1} h g=g_{2}^{-1} p^{-1} g_{1}^{-1} g_{2}^{-1} p_{h} g_{2} g_{1} p g_{2}$.

Since $b$ is equal to $b^{\prime}$ modulo Burnside relations and $b$ is $\psi$-trivial, we can conclude by Lemma 4.20(ii) that $g_{1}^{-1} g_{2}^{-1} p_{h} g_{2} g_{1}$ is equal to a word $p^{\prime \prime} \in P$ modulo Burnside relations. Taking the projection of the equality $\left(g_{2} g_{1}\right)^{-1} p_{h} g_{2} g_{1}=p^{\prime \prime}$ onto the subgroup $P$, we get $P\left(g_{2} g_{1}\right)^{-1} p_{h} P\left(g_{2} g_{1}\right)=$ $p^{\prime \prime}$ modulo Burnside relations, where $P\left(g_{2} g_{1}\right)$ is the $P$-projection of $g_{2} g_{1}$. Therefore $P\left(g_{2} g_{1}\right)^{-1}\left(g_{2} g_{1}\right)$ centralizes $p_{h}$ modulo Burnside relations. Since the centralizers of $p_{h}$ in the free Burnside quotient of $P$ and $P * Q$ have order $n$ (by Lemma 4.18), these centralizers coincide. Hence $P\left(g_{2} g_{1}\right)^{-1} g_{2} g_{1}$ belongs to $P$ modulo Burnside relations, so $g_{2} g_{1} \in P$ modulo Burnside relations. Then $g_{2} g g_{2}^{-1} \in$ $P, g_{2} h g_{2}^{-1}=g_{2} g_{2}^{-1} p_{h} g_{2} g_{2}^{-1}=p_{h} \in P$ modulo Burnside relations, which is impossible by our assumption. 


\section{The presentation of the group}

In this section, we define an $S$-machine consisting of a set of (positive) $S$-rules $\mathbb{S}^{+}$. Using this $S$-machine, we define our groups $\mathcal{H}$ (Theorem 1.3).

Fix some numbers first. We use the same odd number $n>>1$ as in the previous sections. Let $N>n$, and let $m=m(n)$ be the number which exists by Theorem 4.4.

\subsection{S-machines and their interpretation}

We start with definition of the generating set $\mathbf{X}$ of the group $H$ and the alphabet of the $S$-machine.

Let $\mathcal{A}$ be the set $\left\{a_{1}, \ldots, a_{m}\right\}$. Let $\Omega$ be the set $\{0, \ldots, 2 n+1\}$.

The set $\mathbb{S}^{+}$that we shall define in the next section, will be the union of disjoint sets $\mathbb{S}_{\omega}^{+}, \omega \in \Omega$, and the set $\left\{\mathbf{c}_{0}, \ldots, \mathbf{c}_{2 n}\right\}$ called the set of connecting rules. For each $\omega \in\{0, \ldots, 2 n\}$, the set $\mathbb{S}_{\omega}^{+}$will consist of $m$ rules, one for each letter in $\mathcal{A}$. The rules from $\cup_{\omega=0}^{2 n} \mathbb{S}_{\omega} \cup\left\{\mathbf{c}_{0}, \ldots, \mathbf{c}_{2 n}\right\}$ will be called working rules.

The rules from $\mathbb{S}_{2 n+1}^{+}$will be called cleaning rules. The set $\mathbb{S}_{2 n+1}^{+}$will be in one-to-one correspondence with the set of all working rules (thus the set of cleaning rules and the set of working rules are disjoint but have the same cardinality).

Consider the alphabet

$$
\mathbf{K}=\{\lambda(i, \omega), \kappa(i), \rho(i), \vec{\kappa}(i, j, \omega), \overleftarrow{\kappa}(i, j, \omega), i=1, \ldots, N, j=1, \ldots, n, \omega \in \Omega\}
$$

Elements of $\mathbf{K}$ will be called $\mathbf{K}$-letters. Let also $\overline{\mathbf{K}}$ be the set of letters obtained from $\mathbf{K}$ by removing the $\Omega$-coordinate $\omega$, and let us call the corresponding map from $\mathbf{K}$ to $\overline{\mathbf{K}}$ the $\overline{\mathbf{K}}$-projection. The preimage of every letter $z \in \overline{\mathbf{K}}$ under the $\overline{\mathbf{K}}$-projection will be denoted by $\overline{\mathbf{K}}(z)$. For every $z \in(\overline{\mathbf{K}})^{-1}$ we denote $\overline{\mathbf{K}}\left(z^{-1}\right)^{-1}$ by $\overline{\mathbf{K}}(z)$. Sometimes it will be convenient to assume that letters with $\overline{\mathbf{K}}$-projection $\kappa(i)$ or $\rho(i)$ also have $\Omega$-coordinates but we identify all letters $\kappa(i, \omega)$ with $\kappa(i)$ and all letters $\rho(i, \omega)$ with $\rho(i)$.

If $U$ is any word containing letters from $\overline{\mathbf{K}}$, and $\omega \in \Omega$, then we define $U(\omega)$ as the word, obtained from $U$ by replacing every letter $z$ from $\overline{\mathbf{K}} \backslash\{\kappa(i), \rho(i), i=1, \ldots, N\}$ by $z(\omega)$ (in other words, we add the coordinate $\omega$ to each letter from $\overline{\mathbf{K}}$ in $U$ except $\kappa(i), \rho(i))$. In this case we shall call $\omega$ the $\Omega$-coordinate of $U(\omega)$.

For every $z \in \overline{\mathbf{K}} \backslash\{\lambda(i), \rho(i), \kappa(j), 1 \leq i \leq N, j=2, \ldots, N\}$ consider a copy $A(z)$ of the alphabet $\mathcal{A}$. For each $z \in\{\lambda(i), \rho(i), 1 \leq i \leq N\}$, consider the set $\underline{A(z)}$ which is a copy of the set of working rules: with every working rule $\tau$ we associate a letter $\overline{\tau(z)}$ in $A(z)$ (all sets $A(z)$ are disjoint). Notice that we have not defined the sets $A(\kappa(j)), j=2, \ldots, N$. For convenience we assume that all these sets are empty.

Let $\mathbf{A}$ be the union of all the sets $A(z), z \in \overline{\mathbf{K}}$. Elements of this set will be called A-letters.

Consider also the set of $\mathbf{R}$-letters $\mathbf{R}=\left\{r(\tau, z), \tau \in \mathbb{S}^{+}, z \in \overline{\mathbf{K}}\right\}$.

Now let

$$
\mathbf{X}=\mathbf{K} \cup \mathbf{A} \cup \mathbf{R}
$$

This is the generating set of our group $\mathcal{H}$. We rank some of the letters in $\mathbf{X}$ in the following way: $\mathbf{K}>\mathbf{R}>\mathbf{A}$, that is letters from $\mathbf{K}$ have rank higher than all other letters, etc. For every word over $\mathbf{X}$ we can consider its $\mathbf{K}$-length, etc.

Consider the following word $\Lambda$ over $\overline{\mathbf{K}}$ : 


$$
\begin{aligned}
& \lambda(1) \kappa(1) \rho(1) \vec{\kappa}(1,1) \overleftarrow{\kappa}(1,1) \ldots \vec{\kappa}(1, n) \overleftarrow{\kappa}(1, n) \ldots \\
& \lambda(N) \kappa(N) \rho(N) \vec{\kappa}(N, 1) \overleftarrow{\kappa}(N, 1) \ldots \vec{\kappa}(N, n) \overleftarrow{\kappa}(N, n)
\end{aligned}
$$

The word $\Lambda(0)$ will be called the $h u b$.

We define a circular order on $\overline{\mathbf{K}}$ according to the appearance in $\Lambda$ if we consider the word $\Lambda$ as written on a circle. For every $z \in \overline{\mathbf{K}}$ let $z_{-}$be the letter immediately preceding $z$ in our order, and let $z_{+}$be the letter next to $z$ in that order. We extend that order also on letters from $\overline{\mathbf{K}}^{-1}$. If $z \in \overline{\mathbf{K}}$ then $\left(z^{-1}\right)_{-} \equiv\left(z_{+}\right)^{-1},\left(z^{-1}\right)_{+} \equiv\left(z_{-}\right)^{-1}$. We define a corresponding circular order on $\mathbf{K} \cup \mathbf{K}^{-1}$ :

$$
z(\omega)_{+} \equiv z_{+}(\omega)
$$

For every $z \in \overline{\mathbf{K}}^{-1}$ we set $A(z)=A\left(z_{-}^{-1}\right)$. Also by definition for every $\bar{z} \in \mathbf{K}$ with $\overline{\mathbf{K}}$-projection $z$ we let $A\left(\bar{z}^{ \pm 1}\right)=A\left(z^{ \pm 1}\right)$.

The language of admissible words consists of all words of the form $y_{1} u_{1} y_{2} u_{2} \ldots y_{t}$ where $y_{i} \in \mathbf{K}$, $u_{i}$ are words in $A\left(y_{i}\right), i=1,2, \ldots, t-1$, and for every $i=1,2 \ldots$, either $y_{i+1} \equiv\left(y_{i}\right)_{+}$or $y_{i+1} \equiv y_{i}^{-1}$. Thus all $\mathbf{K}$-letters in an admissible word have the same $\Omega$-coordinates. (Notice that $\Lambda(\omega)$ is an admissible word for every $\omega$.) The subword $y_{i} u_{i} y_{i+1}$ is called the $y_{i}$-sector of the admissible word, $i=1,2 \ldots$. Two admissible words $y_{1} u_{1} y_{2} u_{2} \ldots y_{t}$ and $y_{1}^{\prime} u_{1}^{\prime} y_{2}^{\prime} u_{2}^{\prime} \ldots y_{t^{\prime}}^{\prime}$ are considered equal if $t=t^{\prime}$, $y_{i} \equiv y_{i}^{\prime}, i=1, \ldots, t$, and $u_{i}=u_{i}^{\prime}, i=1, \ldots, t-1$, in the free Burnside group (later on we will identify these admissible words if $y_{i} \equiv y_{i}^{\prime}$ and $u_{i}=u_{i}^{\prime}$ in the group $H_{k a}^{*}$ defined below).

The set of defining relations of the group $\mathcal{H}$ consists of the hub $\Lambda(0)$ and relations corresponding to the $S$-rules. We are going to present these relations now.

We start with defining the set of $S$-rules (an $S$-machine in the terminology of [36]) and the corresponding defining relations.

Each rule means simultaneous replacing certain subwords in admissible words by other subwords (the rule specifies the subwords which need be replaced and the replacements).

We shall write a rule $\tau$ in the form $\left[z_{1} \rightarrow v\left(\left(z_{1}\right)_{-}\right) z_{1} u\left(z_{1}\right), \ldots, z_{s} \rightarrow v\left(\left(z_{s}\right)_{-}\right) z_{s} u\left(z_{s}\right) ; \omega \rightarrow \omega^{\prime}\right]$ where $z_{i} \in \overline{\mathbf{K}}$, each of $u(z), v(z)$ is either a letter from $A(z)^{ \pm 1}$ or empty, $z \in \overline{\mathbf{K}}$.

Some of the arrows in a rule can have the form $\stackrel{\ell}{\rightarrow}$. This means that the rule can be applied if the corresponding sector does not have A-letters in it. We shall say that the corresponding sectors are locked by the rule and the rule locks these sectors. If a $z$-sector is locked by the rule, $u(z)$ and $v(z)$ must be empty.

If $z \in \overline{\mathbf{K}}$ does not appear in this rule then we set $u(z), v\left(z_{-}\right)$to be empty. Thus with every rule $\tau$ and every $z \in \overline{\mathbf{K}}$ we associate two words over $\mathbf{A}: u(z)$ and $v(z)$. We can extend this definition to $z \in \overline{\mathbf{K}}^{-1}$ by saying that $v\left(z^{-1}\right) \equiv u(z)^{-1}, u\left(z^{-1}\right) \equiv v\left(z_{-}\right)^{-1}$.

If for some $z \in \overline{\mathbf{K}}, u(z)$ is not empty then we call $\tau$ left active for $z$-sectors, if $v(z)$ is not empty then $\tau$ is right active for $z$-sectors.

If $\tau$ is a rule as above then for every $z \in \overline{\mathbf{K}} \cup \overline{\mathbf{K}}^{-1}$ we define two words $L(\tau, z) \equiv r\left(\tau, z_{-}\right) v\left(z_{-}\right)$ and $R(\tau, z) \equiv r(\tau, z) u(z)^{-1}$. If $h \equiv \tau_{1} \tau_{2} \ldots \tau_{t}$ is a word in the alphabet $\mathbb{S}$, we set $L(h, z) \equiv$ $L\left(\tau_{1}, z\right) L\left(\tau_{2}, z\right) \ldots L\left(\tau_{t}, z\right), R(h, z) \equiv R\left(\tau_{1}, z\right) R\left(\tau_{2}, z\right) \ldots R\left(\tau_{t}, z\right)$.

The rule $\tau$ is applicable to a one-letter admissible word $y$ if and only if the $\Omega$-coordinate of $y$ is $\omega$.

Let $W \equiv y_{1} u y_{2}$ be an admissible word with two K-letters. The following two conditions must hold for $\tau$ to be applicable to $W$ :

(i) $y_{1}$ and $y_{2}$ belong to $\overline{\mathbf{K}}(\omega)$; 
(ii) if $y_{1}$ is one of the letters $z_{i}, i=1, \ldots, s, \tau$ locks $y_{1}$-sectors then $u$ must be empty.

If conditions (i) and (ii) hold then the result of the application of $\tau$ to $W$ is the word

$$
y_{1}^{\prime} u\left(y_{1}\right) u v\left(\left(y_{2}\right)_{-}\right) y_{2}^{\prime}
$$

where $y_{i}^{\prime}$ are obtained from $y_{i}$ by replacing the $\Omega$-coordinate $\omega$ by $\omega^{\prime}$ (recall that $\tau$ contains the instruction $\left.\omega \rightarrow \omega^{\prime}\right)$.

If $W \equiv y_{1} u_{1} y_{2} \ldots u_{t} y_{t}$ is an admissible word with $t \geq 2 \overline{\mathbf{K}}(\omega)$-letters then the rule $\tau$ is applicable to $W$ if and only if it is applicable to every sector of $W$. In order to apply an $S$-rule to $W$ we apply it to every sector of $W$.

Let $W^{\prime}$ be the resulting word. Notice that $W^{\prime}$ is clearly again an admissible word.

With every rule $\tau=\left[z_{1} \rightarrow v\left(\left(z_{1}\right)_{-}\right) z_{1} u\left(z_{1}\right), \ldots, z_{s} \rightarrow v\left(\left(z_{s}\right)_{-}\right) z_{s} u\left(z_{s}\right) ; \omega \rightarrow \omega^{\prime}\right]$ we associate the inverse rule $\left.\tau^{-1}=\left[z_{1} \rightarrow v\left(\left(z_{1}\right)_{-}\right)^{-1} z_{1} u_{(} z_{1}\right)^{-1}, \ldots, z_{s} \rightarrow v\left(\left(z_{s}\right)_{-}\right)^{-1} z_{s} u\left(z_{s}\right)^{-1} ; \omega^{\prime} \rightarrow \omega\right]$. Clearly if $\tau$ is applicable to an admissible word $W$ and $W^{\prime}$ is the result of application of $\tau$ to $W$, then $\tau^{-1}$ is applicable to $W^{\prime}$ and $W$ is the result of application of $\tau^{-1}$ to $W^{\prime}$.

The rules from $\mathbb{S}^{+}$, described below, will be called positive, the inverses of these rules will be called negative.

Now let us describe the procedure of converting an $S$-rule into a set of relations. After that we shall present the list of rules $\mathbb{S}$ corresponding to our group $\mathcal{H}$.

Let $\tau=\left[z_{1} \rightarrow v\left(\left(z_{1}\right)_{-}\right) z_{1} u\left(z_{1}\right), \ldots, z_{s} \rightarrow v\left(\left(z_{s}\right)_{-}\right) z_{s} u\left(z_{s}\right) ; \omega \rightarrow \omega^{\prime}\right]$ be an $S$-rule. Then for every $z \in \overline{\mathbf{K}}$, we introduce a relation

$$
r\left(\tau, z_{-}\right)^{-1} z(\omega) r(\tau, z)=v\left(z_{-}\right) z\left(\omega^{\prime}\right) u(z) .
$$

If (and only if) $\tau$ does not lock $z$-sectors, we also include all relations of the form

$$
r(\tau, z) b=b r(\tau, z), b \in A(z) .
$$

For every $z \in \overline{\mathbf{K}}$ let $\mathbf{R}(z)$ denote the set $\{r(\tau, z) \mid \tau \in \mathbb{S}\}$.

To make formulas look less frightening let us agree on the following:

In every word of the form $u_{a r} z v_{a r}$ appearing in an $S$-rule or in a defining relation of our group, or in an admissible word, where $z \in \overline{\mathbf{K}}(\omega), u_{a r}, v_{a r}$ are words in the alphabet $\mathbf{A} \cup \mathbf{R}$, we shall always assume that the letters in $u_{a r}$ are from $A\left(z_{-}\right) \cup \mathbf{R}\left(z_{-}\right)$only, and the letters in $v_{a r}$ are from $A(z) \cup \mathbf{R}(z)$ only. So we shall omit the $\overline{\mathbf{K}}$-coordinates in these words. We shall also omit the $\Omega$-coordinate in $\mathrm{K}$-letters if the value of it is clear.

Example. Suppose, for example, that the rule $\tau$ has the form

$$
[\kappa(1) \rightarrow \kappa(1) a, \vec{\kappa}(1,1) \rightarrow \vec{\kappa}(1,1) a, \overleftarrow{\kappa}(1,2) \stackrel{\ell}{\rightarrow} a \overleftarrow{\kappa}(1,2), 1 \rightarrow 2]
$$

(this rule is not in $\mathbb{S}$, we use it just as an example). Then the set of relations corresponding to this rule consists of the following relations.

- $r(\tau)^{-1} \kappa(1) r(\tau)=\kappa(1) a$;

- $r(\tau)^{-1} \vec{\kappa}(1,1,1) r(\tau)=\vec{\kappa}(1,1,2) a$; 


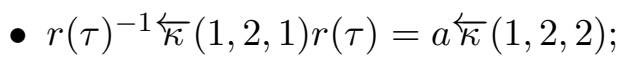

- $r(\tau)^{-1} z(1) r(\tau)=z(2)$ for every $z \in \overline{\mathbf{K}}, z \not \equiv \kappa(1), \vec{\kappa}(1,1)$.

- $r(\tau) b=\operatorname{br}(\tau)$ for every $b \in A(z)$ and $z \not \equiv \overleftarrow{\kappa}(1,2)$.

Notice that, for example in the relation $r(\tau)^{-1} \vec{\kappa}(1,1,1) r(\tau)=\vec{\kappa}(1,1,2) a$, the two letters $r(\tau)$ are different according to our agreement: the first letter is $r(\tau, \kappa(1))$ and the second letter is $r(\tau, \rho(1))$. This rule $\tau$ is left active for $\kappa(1)$-sectors and for $\vec{\kappa}(1,1)$-sectors, right active for $\overleftarrow{\kappa}(1,2)$-sectors, and locking for $\overleftarrow{\kappa}(1,1)$-sectors.

Here is one of the main properties of the systems of relations corresponding to $S$-rules. The lemma immediately follows from definitions.

Lemma 5.1. Let $W$ be an admissible word, starting with $z \in \mathbf{K}$ and ending with $z^{\prime} \in \mathbf{K}$, let $\tau=\left[z_{1} \rightarrow v\left(\left(z_{1}\right)_{-}\right) z_{1} u\left(z_{1}\right), \ldots, z_{s} \rightarrow v\left(\left(z_{s}\right)_{-}\right) z_{s} u\left(z_{s}\right) ; \omega \rightarrow \omega^{\prime}\right]$ be an $S$-rule applicable to $W$ (some of the arrows $\rightarrow$ may be of the form $\stackrel{\ell}{\rightarrow})$. Then:

1. $L(\tau, z)^{-1} W R\left(\tau, z^{\prime}\right)$ is equal modulo the relations corresponding to $\tau$ to the admissible word $W^{\prime}$ obtained by applying $\tau$ to $W$.

2. If $\tau$ locks z-sectors then the z-sectors of $W$ and $W^{\prime}$ have trivial retraction to the subgroup $\langle\mathbf{A}\rangle$ in the free group generated by $\mathbf{A} \cup \mathbf{K}$.

3. For every $z \in \overline{\mathbf{K}}, L(\tau, z) z\left(\omega^{\prime}\right)=z(\omega) R(\tau, z)$ is one of the defining relations corresponding to $\tau$.

\subsection{The $S$-machine $\mathbb{S}$}

Now let us define our collection of $S$-rules $\mathbb{S}^{+}$. As we have announced in the Section 5.1, the set $\mathbb{S}^{+}$will be divided into $2 n+3$ subsets:

$$
\mathbb{S}_{0}^{+}, \ldots, \mathbb{S}_{2 n+1}^{+},\left\{\mathbf{c}_{i} ; i=0, \ldots, 2 n\right\} .
$$

The sets $\mathbb{S}_{i}$ will be called steps, $\mathbf{c}_{i}$ will be called connecting rules. Rules from $\mathbb{S}_{2 n+1}$ are called cleaning rules, other rules are called working.

In each set $\mathbb{S}_{i}^{+}, i \leq 2 n$, each rule corresponds to a letter $a \in \mathcal{A}$, it will be denoted by $\tau(i, a)$.

The rule $\tau(0, a)$ in the set $\mathbb{S}_{0}^{+}$is the following

$$
\left[\begin{array}{l}
\left.\lambda(i) \stackrel{\ell}{\rightarrow} \lambda(i), \kappa(i) \stackrel{\ell}{\rightarrow} \kappa(i), \rho(i) \stackrel{\ell}{\rightarrow} \rho(i), \vec{\kappa}(i, j) \rightarrow \vec{\kappa}(i, j) a^{-1}, \overleftarrow{\kappa}(i, j) \stackrel{\ell}{\rightarrow} \overleftarrow{\kappa}(i, j),\right] \\
i=1, \ldots, N, j=1, \ldots, n ; 0 \rightarrow 0
\end{array}\right.
$$

This rule inserts copies of the letter $a^{-1}$ to the right of $\vec{\kappa}(i, j)$. A sequence

$$
\tau\left(0, a_{i_{1}}\right)^{ \pm 1}, \ldots, \tau\left(0, a_{i_{s}}\right)^{ \pm 1}
$$

of these rules inserts copies of the word $\left(a_{i_{1}}^{ \pm 1} \ldots a_{i_{s}}^{ \pm 1}\right)^{-1}$ in $\vec{\kappa}(i, j)$-sectors. These rules lock all other sectors. The connecting rule $\mathbf{c}_{0}$ has the form 


$$
[\lambda(i) \stackrel{\ell}{\rightarrow} \lambda(i), \kappa(i) \stackrel{\ell}{\rightarrow} \kappa(i), \rho(i) \stackrel{\ell}{\rightarrow} \rho(i), \overleftarrow{\kappa}(i, j) \stackrel{\ell}{\rightarrow} \overleftarrow{\kappa}(i, j), i=1, \ldots, N, j=1, \ldots, n ; 0 \rightarrow 1] .
$$

It locks all sectors except for $\vec{\kappa}(i, j)$-sectors.

The rule $\tau(1, a)$ has the form

$$
\left[\begin{array}{l}
\kappa(i) \stackrel{\ell}{\rightarrow} \overline{\tau(1, a)} \kappa(i), \vec{\kappa}(i, 1) \rightarrow \overline{\tau(1, a)} \vec{\kappa}(i, 1) a \\
\vec{\kappa}(i, j) \rightarrow \vec{\kappa}(i, j) a, \overleftarrow{\kappa}(i, j) \rightarrow \overleftarrow{\kappa}(i, j) a \\
1 \leq i \leq N, 2 \leq j \leq n ; 1 \rightarrow 1
\end{array}\right]
$$

This rule moves letters from $\vec{\kappa}(i, j)$-sectors to $\overleftarrow{\kappa}(i, j)$-sectors (i.e. the rule removes a letter from $\vec{\kappa}(i, j)$-sectors, and adds a copy of that letter to each of the $\overleftarrow{\kappa}(i, j)$-sectors). It writes a copy of itself in the $\lambda(i)$-sectors and $\rho(i)$-sectors. It locks all $\kappa(i)$-sectors.

The connecting rule $\mathbf{c}_{1}$ has the form

$$
\left[\begin{array}{l}
\kappa(i) \stackrel{\ell}{\rightarrow} \overline{\mathbf{c}_{1}} \kappa(i), \vec{\kappa}(i, 1) \stackrel{\ell}{\rightarrow} \overline{\mathbf{c}_{1}} \vec{\kappa}(i, 1), \vec{\kappa}(i, j) \stackrel{\ell}{\rightarrow} \vec{\kappa}(i, j), \\
1 \leq i \leq N, 2 \leq j \leq n ; 1 \rightarrow 2
\end{array}\right]
$$

It writes a copy of itself in the $\lambda(i)$-sectors and $\rho(i)$-sectors. It locks $\vec{\kappa}(i, j)$-sectors and $\kappa(i)$-sectors.

For every $t=2, \ldots, n$ and every $a \in \mathcal{A}$ the rule $\tau(2 t-1, a)$ has the form:

$$
\left[\begin{array}{l}
\kappa(1) \rightarrow \overline{\tau(2 t-1, a)} \kappa(1), \kappa\left(i^{\prime}\right) \stackrel{\ell}{\rightarrow} \overline{\tau(2 t-1, a)} \kappa\left(i^{\prime}\right) \\
\vec{\kappa}(i, 1) \stackrel{\ell}{\rightarrow} \overline{\tau(2 t-1, a)} \vec{\kappa}(i, 1), \overleftarrow{\kappa}(i, 1) \stackrel{\ell}{\rightarrow} \overleftarrow{\kappa}(i, 1) \\
\vec{\kappa}(i, s) \stackrel{\ell}{\rightarrow} \vec{\kappa}(i, s), \overleftarrow{\kappa}(i, s) \stackrel{\ell}{\rightarrow} \overleftarrow{\kappa}(i, s), \vec{\kappa}(i, j) \rightarrow \overleftrightarrow{\kappa}(i, j) a, \overleftarrow{\kappa}(i, j) \rightarrow \overleftarrow{\kappa}(i, j) a, \\
1 \leq i \leq N, 2 \leq i^{\prime} \leq N, 2 \leq s<t, t \leq j \leq n ; \quad 2 t-1 \rightarrow 2 t-1
\end{array}\right]
$$

This rule moves letters from $\vec{\kappa}(i, j)$-sectors to $\overleftarrow{\kappa}(i, j)$-sectors, $j=t, \ldots, n$, and writes a copy of itself in the $\lambda(i)$-sectors and $\rho(i)$-sectors. It locks $\kappa(i)$-sectors, $i \geq 2$, all $\vec{\kappa}(i, j)$-sectors, $j<t$, and all $\overleftarrow{\kappa}(i, j)$-sectors, $j<t$

The connecting rule $\mathbf{c}_{2 t-1}$ has the form

$$
\left[\begin{array}{l}
\kappa(1) \rightarrow \overline{\mathbf{c}_{2 t-1}} \kappa(1), \kappa\left(i^{\prime}\right) \stackrel{\ell}{\rightarrow} \overline{\mathbf{c}_{2 t-1}} \kappa\left(i^{\prime}\right) \\
\vec{\kappa}(i, 1) \stackrel{\ell}{\rightarrow} \overline{\mathbf{c}_{2 t-1}} \vec{\kappa}(i, 1), \overleftarrow{\kappa}(i, 1) \stackrel{\ell}{\rightarrow} \overleftarrow{\kappa}(i, 1) \\
\vec{\kappa}(i, j) \stackrel{\ell}{\rightarrow} \vec{\kappa}(i, j), \overleftarrow{\kappa}(i, s) \stackrel{\ell}{\rightarrow} \overleftarrow{\kappa}(i, s) \\
1 \leq i \leq N, 2 \leq i^{\prime} \leq N, 2 \leq s<t, 2 \leq j \leq n ; 2 t-1 \rightarrow 2 t
\end{array}\right]
$$

It locks $\kappa(i)$-sectors, $i \geq 2$, all $\vec{\kappa}(i, j)$-sectors, and all $\overleftarrow{\kappa}(i, j)$-sectors, $j<t$. It also writes a copy of itself in the $\lambda(i)$-sectors and $\rho(i)$-sectors.

For every $t=1, \ldots, n$ the rule $\tau(2 t, a)$ has the form:

$$
\left[\begin{array}{l}
\kappa(1) \rightarrow \overline{\tau(2 t, a)} \kappa(1) a^{-1}, \vec{\kappa}(i, 1) \stackrel{\ell}{\rightarrow} \overline{\tau(2 t, a)} \vec{\kappa}(i, 1), \overleftarrow{\kappa}(i, 1) \stackrel{\ell}{\rightarrow} \overleftarrow{\kappa}(i, 1), \\
\kappa\left(i^{\prime}\right) \stackrel{\ell}{\rightarrow} \overline{\tau(2 t, a)} \kappa\left(i^{\prime}\right), \vec{\kappa}(i, s) \stackrel{\ell}{\rightarrow} \vec{\kappa}(i, s), \overleftarrow{\kappa}\left(i, s^{\prime}\right) \stackrel{\ell}{\rightarrow} \overleftarrow{\kappa}\left(i, s^{\prime}\right), \vec{\kappa}(i, j) \rightarrow \vec{\kappa}(i, j) a^{-1}, \\
\overleftarrow{\kappa}\left(i, j^{\prime}\right) \rightarrow \overleftarrow{\kappa}\left(i, j^{\prime}\right) a^{-1} \\
1 \leq i \leq N, 2 \leq i^{\prime} \leq N, 2 \leq s \leq t, t<j \leq n, 1 \leq s^{\prime}<t, t \leq j^{\prime} \leq n ; 2 t \rightarrow 2 t
\end{array}\right]
$$


This rule moves letters from $\overleftarrow{\kappa}(i, t)$-sector to $k(1)$-sector, letters from $\overleftarrow{\kappa}(i, j)$-sectors, $j>t$, to $\vec{\kappa}(i, j)$-sectors, and writes a copy of itself in the $\lambda(i)$-sectors and $\rho(i)$-sectors. It locks all other sectors.

The connecting rule $\mathbf{c}_{2 t}, t=1, \ldots, n-1$, has the form

$$
\left[\begin{array}{l}
\kappa(i) \stackrel{\ell}{\rightarrow} \overline{\mathbf{c}_{2 t}} \kappa(i), \overleftarrow{\kappa}(i, 1) \stackrel{\ell}{\rightarrow} \overleftarrow{\kappa}(i, 1) \\
\vec{\kappa}(i, s) \stackrel{\ell}{\rightarrow} \vec{\kappa}(i, s), \overleftarrow{\kappa}(i, j) \stackrel{\ell}{\rightarrow} \overleftarrow{\kappa}(i, j) \\
1 \leq i \leq N, 2 \leq s \leq t, 1 \leq j \leq n ; \quad 2 t \rightarrow 2 t+1
\end{array}\right]
$$

It writes a copy of itself in the $\lambda(i)$-sectors and $\rho(i)$-sectors. It locks all $\kappa(i)$-sectors, $i>1$, all $\overleftarrow{\kappa}(i, j)$-sectors and all $\vec{\kappa}(i, j)$-sectors, $i \leq t$.

The connecting rule $\mathbf{c}_{2 n}, t=1, \ldots, n-1$, has the form

$$
\left[\begin{array}{l}
\kappa(i) \stackrel{\ell}{\rightarrow} \overline{\mathbf{c}_{2 n}} \kappa(i), \vec{\kappa}(i, 1) \stackrel{\ell}{\rightarrow} \overline{\mathbf{c}_{2 n}} \vec{\kappa}(i, 1), \overleftarrow{\kappa}(i, j) \stackrel{\ell}{\rightarrow} \overleftarrow{\kappa}(i, j) \\
\vec{\kappa}\left(i, j^{\prime}\right) \stackrel{\ell}{\rightarrow} \vec{\kappa}\left(i, j^{\prime}\right) \\
1 \leq i \leq N, 1 \leq j \leq n, 2 \leq j^{\prime} \leq n ; \quad 2 n \rightarrow 2 n+1
\end{array}\right]
$$

It writes a copy of itself in the $\lambda(i)$-sectors and $\rho(i)$-sectors. It locks all other sectors.

The cleaning rules are in one-to-one correspondence with working rules. For every working rule $r$, the corresponding cleaning rule $\tau(2 n+1, r)$ has the form

$$
\left[\begin{array}{l}
\kappa(i) \stackrel{\ell}{\rightarrow} \bar{r} \kappa(i), \vec{\kappa}(i, 1) \stackrel{\ell}{\rightarrow} \bar{r} \vec{\kappa}(i, 1), \overleftarrow{\kappa}(i, 1) \stackrel{\ell}{\rightarrow} \overleftarrow{\kappa}(i, 1) \\
\vec{\kappa}(i, j) \stackrel{\ell}{\rightarrow} \vec{\kappa}(i, j), \overleftarrow{\kappa}(i, j) \stackrel{\ell}{\rightarrow} \overleftarrow{\kappa}(i, j), 1 \leq i \leq N, 2 \leq j \leq n ; 2 n+1 \rightarrow 2 n+1
\end{array}\right]
$$

These rules remove the content of $\lambda(i, j)$-sectors and $\rho(i, j)$-sectors (which contain copies of the history of the computation) and locks all other sectors.

Notice that only connecting rules change the $\Omega$-coordinates of $\mathbf{K}$-letters. Notice also that for every $a \in \mathcal{A}$ and every $i=1, \ldots, 2 n$, one can define the rule $\tau\left(i, a^{-1}\right)$ as before. Similarly for every working rule $r$ one can define $\tau\left(2 n+1, r^{-1}\right)$. It is easy to see that

$$
\tau\left(i, a^{-1}\right)=\tau(i, a)^{-1}
$$

The following picture (Figure 13) shows which rules of the $S$-machine $\mathbb{S}$ are locking, active or neither for which sectors of admissible words. 

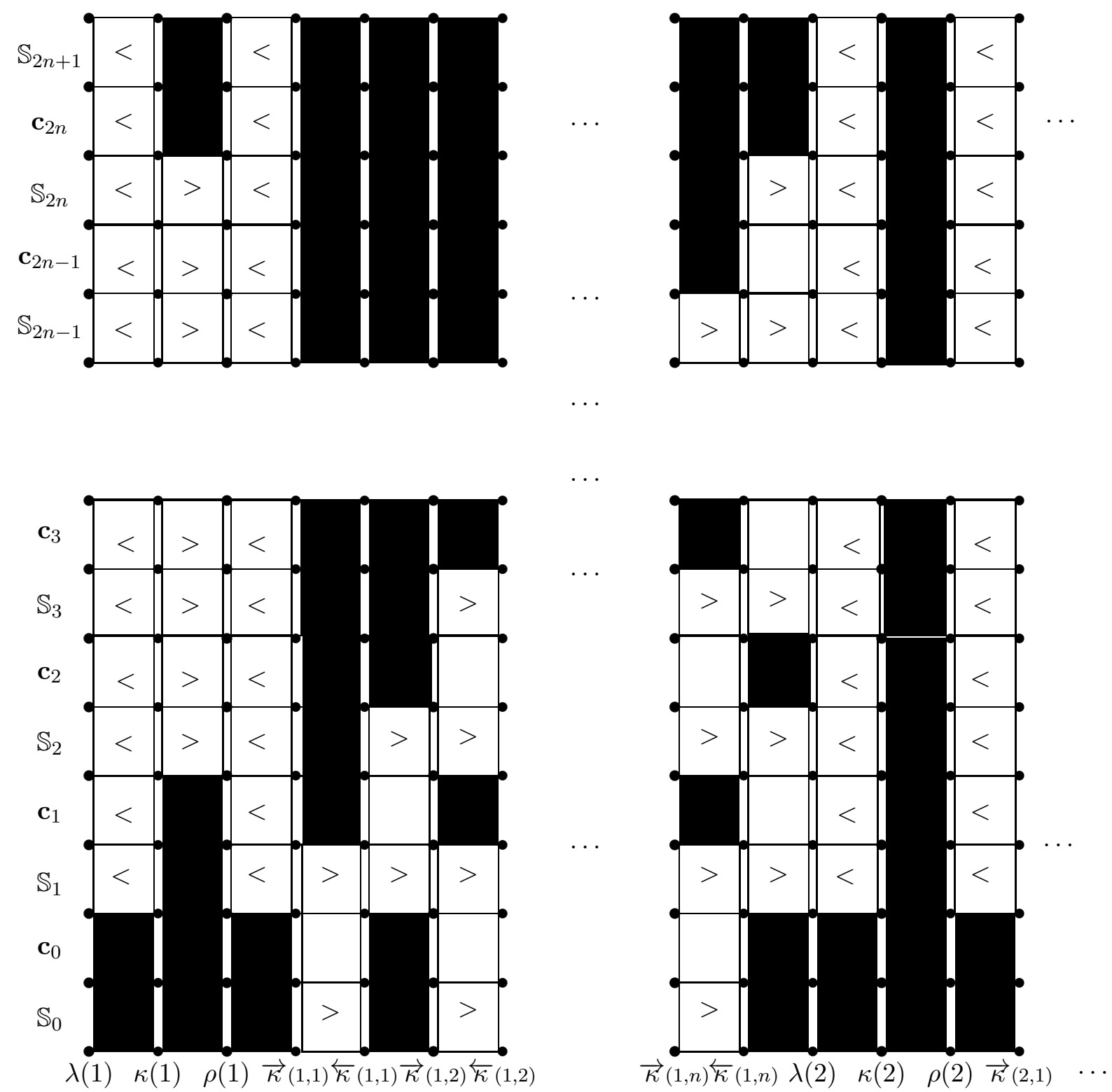

Fig. 13.

A filled box means that the sector is locked, a box with ">" in it means that the rules are left active for that sector, a box with " $<$ " in it means that the rules are right active, and an empty box means that the rules from the corresponding set of $S$-rules are neither active for that sector nor locking it. For example, this picture shows that rules from $\mathbb{S}_{2}$ are right active for $\lambda(i)$-sectors and $\rho(i)$-sectors, lock $\vec{\kappa}(1,1)$-sectors but are left active for $\vec{\kappa}(1,2)$-sectors. It also shows that $\kappa(i)$-sectors, $i \geq 2$, are locked by all rules of $\mathbb{S}$. 


\subsection{The presentation}

The set of relations corresponding to the rules from $\mathbb{S}$ will be denoted by $Z(\mathbb{S})$. By $Z(\mathbb{S}, \Lambda)$ we shall denote the set $Z(\mathbb{S})$ together with the hub $\Lambda(0)$.

Our main result is that the group $\mathcal{H}$ defined by the set of relations $Z(\mathbb{S}, \Lambda)$ satisfies the two conditions of Theorem 1.3.

\subsection{The subgroup $\langle\mathcal{A}\rangle$ of $\mathcal{H}$ is a torsion group of exponent $n$}

For every group word $u$ in the alphabet $\mathcal{A}$ and every K-letter $z \notin\{\lambda(i), \rho(i, 1), i=1, \ldots, N\}$ we denote a copy of $u$ in the alphabet $A(z)$ by $u(z)$. We may identify $\mathcal{A}$ with $A(\kappa(1))$.

Lemma 5.2. For every word $u$ in $\mathcal{A} \cup \mathcal{A}^{-1}$ the relation $u(\kappa(1))^{n}=1$ follows from $Z(\mathbb{S}, \Lambda)$.

Proof. Let $u \equiv a_{i_{1}} a_{i_{2}} \ldots a_{i_{s}}$ be a group word in the alphabet $\mathcal{A}$. Start with the admissible word $\Lambda(0) \lambda(1,0)$ and apply $S$-rules $\tau\left(0, a_{i_{1}}\right), \ldots, \tau\left(0, a_{i_{s}}\right)$ from $\mathbb{S}_{0}$ to $\Lambda(0) \lambda(1,0)$. The resulting word is equal to

$$
\begin{aligned}
& \lambda(1) \kappa(1) \rho(1) \vec{\kappa}(1,1) u^{-1} \overleftarrow{\kappa}(1,1) \vec{\kappa}(1,2) u^{-1} \overleftarrow{\kappa}(1,2) \ldots \lambda(N) \kappa(N) \rho(N) \\
& \vec{\kappa}(N, n) u^{-1} \overleftarrow{\kappa}(N, n) \lambda(1)
\end{aligned}
$$

the $\Omega$-coordinate of each $\mathbf{K}$-letter is 0 .

Applying now the connecting rule $\mathbf{c}_{0}$, we change the $\Omega$-coordinates of all $\mathbf{K}$-letters to 1 .

After that, applying rules $\tau\left(1, a_{i_{s}}\right), \ldots, \tau\left(1, a_{i_{1}}\right)$, we get the word

$$
\begin{aligned}
& \lambda(1) v_{1} \kappa(1) \rho(1) v_{1} \vec{\kappa}(1,1) \overleftarrow{\kappa}(1,1) u \vec{\kappa}(1,2) \ldots \lambda(N) v_{1} \kappa(N) \rho(N) v_{1} \vec{\kappa}(N, 1) \ldots \\
& \vec{\kappa}(N, n) \overleftarrow{\kappa}(N, n) u \lambda(1)
\end{aligned}
$$

(the $\Omega$-coordinates are 1$)$ where $v_{1} \equiv \overline{\tau\left(1, a_{i_{s}}\right)} \ldots \overline{\tau\left(1, a_{i_{1}}\right)}$.

The connecting rule $\mathbf{c}_{1}$ makes all $\Omega$-coordinates 2 and adds letter $\overline{\mathbf{c}_{1}}$ at the end of $v$.

Now we can apply rules $\tau\left(2, a_{i_{1}}\right), \ldots, \tau\left(2, a_{i_{s}}\right), \mathbf{c}_{2}, \tau\left(3, a_{i_{s}}\right), \ldots, \tau\left(3, a_{i_{1}}\right), \mathbf{c}_{3}, \ldots, \tau\left(2 n, a_{i_{1}}\right), \ldots$, $\tau\left(2 n, a_{i_{s}}\right)$, and obtain the following word:

$$
\begin{aligned}
& \lambda(1) v_{2 n} \kappa(1) u^{-n} \rho(1) v_{2 n} \vec{\kappa}(1,1) \overleftarrow{\kappa}(1,1) \ldots \lambda(N) v_{2 n} \kappa(N) \rho(N) v_{2 n} \vec{\kappa}(N, 1) \overleftarrow{\kappa}(N, 1) \\
& \ldots \vec{\kappa}(N, n) \overleftarrow{\kappa}(N, n) \lambda(1)
\end{aligned}
$$

where the $\Omega$-coordinate of every $\mathbf{K}$-letter is $2 n, v_{2 n}$ is a copy of the history of the computation.

Let $W$ be the word (18) without the last letter.

Let $h \equiv \tau\left(0, a_{i_{1}}\right) \ldots \tau\left(2 n, a_{i_{s}}\right)$ be the string of all rules from $\mathbb{S}$ applied so far to $\Lambda(0) \lambda(1)$. Then by Lemma 5.1 (part 3), we have the following equality:

$$
L(h, \lambda(1)) W \lambda(1,2 n)=\Lambda(0) \lambda(1,0) R(h, \lambda(1))=\Lambda(0) L(h, \lambda(1)) \lambda(1,2 n) .
$$

So

$$
W=L(h, \lambda(1))^{-1} \Lambda(0) L(h, \lambda(1))=1
$$

modulo $Z(\mathbb{S}, \Lambda)$.

Notice that if we apply connecting rules $\mathbf{c}_{0}, \ldots, \mathbf{c}_{2 n}$ to $\Lambda(0) \lambda(1,0)$, we obtain

$$
\Lambda(2 n+1) \lambda(1,2 n+1)
$$


Now take the word $\Lambda(2 n+1) \lambda(1,2 n+1)$ and apply the cleaning rules $\tau\left(2 n+1, t_{1}\right) \ldots \tau\left(2 n+1, t_{s}\right)$ where $\overline{t_{1}} \ldots \overline{t_{s}} \equiv v_{2 n}$, and the rule $\mathbf{c}_{2 n}^{-1}$. We get the word

$$
\begin{aligned}
& \lambda(1) v_{2 n} \kappa(1) \rho(1) v_{2 n} \vec{\kappa}(1,1) \overleftarrow{\kappa}(1,1) \ldots \lambda(N) v_{2 n} \kappa(N) \rho(N) v_{2 n} \vec{\kappa}(N, 1) \overleftarrow{\kappa}(N, 1) \\
& \ldots \vec{\kappa}(N, n) \overleftarrow{\kappa}(N, n) \lambda(1)
\end{aligned}
$$

with $\Omega$-coordinate $2 n$. Let $W^{\prime}$ be the word (19) without the last letter. Let $h^{\prime}$ be the word of rules applied to $\Lambda(0) \lambda(1,0)$ to get (19). Then by Lemma 5.1 , we have

$$
L\left(h^{\prime}, \lambda(1)\right) W^{\prime} \lambda(1,2 n)=\Lambda(0) \lambda(1,0) R\left(h^{\prime}, \lambda(1)\right)=\Lambda(0) L\left(h^{\prime}, \lambda(1)\right) \lambda(1,2 n)
$$

modulo $Z(\mathbb{S}, \Lambda)$. This implies $W^{\prime}=1$ modulo $Z(\mathbb{S}, \Lambda)$. But the words $W$ and $W^{\prime}$ differ only by the factor $u(\kappa(1))^{-n}$. Thus $u(\kappa(1))^{n}=1$ modulo $Z(\mathbb{S}, \Lambda)$. Hence the $n$-th power of every word over $A(\kappa(1))$ is 1 modulo $Z(\mathbb{S}, \Lambda)$.

\section{Properties of the $S$-machine $\mathbb{S}$}

\subsection{The inverse semigroup $P(\mathbb{S})$}

We fix an arbitrary set $R(\kappa(1))$ of words in the alphabet $A(\kappa(1))=\mathcal{A}$. (We choose $R(\kappa(1))=\emptyset$ for the construction of the group $\mathcal{H}$, but later we should consider arbitrary $R(\kappa(1))$ to prove part $(2)$ of Theorem 1.3.)

Let $H_{a}$ be the group generated by the set $\mathbf{A}$ subject all relations of the form $u^{n}=1$ ( $u$ is any word in $\mathbf{A}$ ) and relations $r=1$ for all $r \in R(\kappa(1))$. The group $H_{a}$ is isomorphic to the free product in the variety of groups of exponent $n$ of subgroups $\langle A(z)\rangle, z \in \overline{\mathbf{K}}$, where all $\langle A(z)\rangle, z \neq \kappa(1)$, are free Burnside groups of exponent $n$, and $\langle A(\kappa(1))\rangle$ is a group of exponent $n$ with additional relators from $R(\kappa(1))$.

Let $H_{k a}^{*}$ be the free product of the group $H_{a}$ and the free group freely generated by the set $\mathbf{K}$.

¿From now on we will not distinguish admissible words which are equal in $H_{k a}^{*}$. Thus a word of the form $y_{1} u_{1} \ldots y_{t}$ where $y_{i} \in \mathbf{K}, u_{i}$ are words in $A\left(z_{i}\right)$, is admissible if for every $i=1, \ldots, t-1$, either $y_{i+1} \equiv\left(y_{i}\right)_{+}$or $y_{i+1} \equiv y_{i}^{-1}$.

If no sector of an admissible word $W$ is equal in $H_{k a}^{*}$ to a word without K-letters then $W$ is called a reduced admissible word. By Lemma 3.3 this property of an admissible word is equivalent to being $\mathbf{K}$-reduced in $H_{k a}^{*}$.

The result of an application of an $S$-rule to an admissible word is again an admissible word.

Let $W^{\prime}$ be a word obtained from $W$ by an application of a rule $\tau$ from $\mathbb{S}$. Then the $\mathbf{K}$-letters in $W$ differ from the corresponding $\mathbf{K}$-letters in $W^{\prime}$ by their $\Omega$-coordinates only.

Therefore we have the following simple fact.

Lemma 6.1. If $W^{\prime}$ is obtained from $W$ by a nonempty sequence of applications of $S$-rules from $\mathbb{S}$ then the word $W^{\prime}$ is also admissible and the $\mathbf{K}$-letters of $W^{\prime}$ differ from the corresponding $\mathbf{K}$-letters in $W$ only by the $\Omega$-coordinate. The $\Omega$-coordinate of $W^{\prime}$ is $\omega$ if and only if the last rule applied was from $\mathbb{S}_{\omega}$ or equal to $\mathbf{c}_{\omega-1}, \mathbf{c}_{\omega}^{-1}$.

Thus every $\tau \in \mathbb{S}$ defines a partial one-to-one transformation of the set of admissible words. Let $P(\mathbb{S})$ be the subsemigroup generated by these partial transformations in the inverse semigroup of all partial one-to-one transformations of the set of admissible words. Then $P(\mathbb{S})$ is an inverse semigroup because for every rule $\tau$ in $\mathbb{S}$, the inverse rule $\tau^{-1}$ is also in $\mathbb{S}$. The zero of $P(\mathbb{S})$ is 
a transformation with empty domain. Removing zero from $P(\mathbb{S})$ we get what is usually called a pseudogroup of partial transformations. Every word in $\mathbb{S}$ induces a partial one-to-one transformation from $P(\mathbb{S})$.

The following obvious lemma is a general property of inverse semigroups [8].

Lemma 6.2. Let a word $h$ over $\mathbb{S}$ be graphically equal to $h_{1} a a^{-1} h_{2}$. Then, considered as a transformation of the set of admissible words, the domain of $h$ is contained in the domain of $h_{1} h_{2}$, but the partial transformations induced by $h$ and $h_{1} h_{2}$ coincide on the domain of $h$. Thus for every word $h$ over $\mathbb{S}$ the domain of the transformation induced by $h$ is contained in the domain of transformation induced by the reduced form of $h$.

Notice that the domain of the reduced form of $h$ may be strictly bigger than the domain of $h$.

If $h$ is a word in $\mathbb{S}$ then denote by $W \cdot h$ ( $W$ is an admissible word), the result of the application of the freely reduced form of $h$ to $W$ (if it exists). Thus the equality $W \cdot h=W^{\prime}$ means that $W$ is in the domain of the freely reduced form of $h$ and the transformation induced by the reduced form of $h$ takes $W$ to $W^{\prime}$.

The following easy but important lemma immediately follows from the definition of application of an $S$-rule to an admissible word.

Lemma 6.3. Let $W$ be an admissible word. Let $h \equiv \tau_{1} \ldots \tau_{t}$ be a word in $\mathbb{S}$. Then the following conditions are equivalent:

(i) W belongs to the domain of $h$;

(ii) Every sector of $W$ belongs to the domain of $h$.

Moreover $W^{\prime}=W \cdot h$ if and only if for every $z$-sector $W_{1}$ of $W$ and z-sector $W_{1}^{\prime}$ of $W^{\prime}$ we have $W_{1}^{\prime}=W_{1} \cdot h, z \in \overline{\mathbf{K}} \cup \overline{\mathbf{K}}^{-1}$.

For every $\tau \in \mathbb{S}$ and $z \in \overline{\mathbf{K}} \cup \overline{\mathbf{K}}^{-1}$ we have defined two words $L(\tau, z)$ and $R(\tau, z)$. These words belong to the free group generated by $\mathbf{A} \cup \mathbf{R}$. Let $L^{a}(\tau, z)$ and $R^{a}(\tau, z)$ be the $\mathbf{A}$-projections of $L(\tau, z)$ and $R(\tau, z)$. For every word $h$ in the alphabet $\mathbb{S}$, we define the words $L(h, z), R(h, z)$, $L^{a}(h, z)$ and $R^{a}(h, z)$ in the natural way $(L(h, z)$ and $R(h, z)$ have been already defined before). Clearly $L^{a}(., z)$ and $R^{a}(., z)$ are homomorphisms from the free semigroup generated by $\mathbb{S}$ into the subgroups $\left\langle A\left(z_{-}\right)\right\rangle$and $\langle A(z)\rangle$ respectively.

We say that a word $w$ is a copy of a word $w^{\prime}$ if $w^{\prime}$ is obtained from $w$ by replacing equal letters for equal letters and different letters for different letters. For example, $x y x$ is a copy of $a b a$.

The following lemma immediately follows from the definition of the words $L(\tau, z)$ and $R(\tau, z)$ and the definition of an application of an $S$-rule.

Lemma 6.4. (i) Let $h$ be a word over $\mathbb{S}$ applicable (in $P(\mathbb{S})$ ) to a one-sector admissible word $z_{1} w z_{2}$ then $z_{1} w z_{2} \cdot h=z_{1}^{\prime}\left(R^{a}\left(h, z_{1}\right)\right)^{-1} w L^{a}\left(h, z_{2}\right) z_{2}^{\prime}$ where $z_{i}^{\prime}$ differs from $z_{i}$ only by the $\Omega$-coordinate, $i=1,2$.

(ii) For every $\omega \in \Omega$ and every $z \in \overline{\mathbf{K}} \cup \overline{\mathbf{K}}^{-1}$ either $L^{a}(\tau, z)$ (resp. $R^{a}(\tau, z)$ ) is empty for every $\tau \in \mathbb{S}_{\omega}$ or $L^{a}(\tau, z)$ (resp. $R^{a}(\tau, z)$ ) is not empty for every $\tau \in \mathbb{S}_{\omega}$. If for some $\tau \in \mathbb{S}$ and some $z \in \overline{\mathbf{K}} \cup \overline{\mathbf{K}}^{-1}, L^{a}(\tau, z)$ is not empty (resp. $R^{a}\left(\tau, z_{+}\right)$is not empty) then for every $\tau \in \mathbb{S}, L^{a}\left(\tau, z_{+}\right)$ is empty (resp. $R^{a}(\tau, z)$ is empty).

(iii) Suppose that either none of the rules appearing in a word $h$ over $\mathbb{S}$ are from $\mathbb{S}_{2 n+1}$ or all of them are from $\mathbb{S}_{2 n+1}$. Then if all rules of a word $h$ over $\mathbb{S}$ are left active for z-sectors for some $z \in \overline{\mathbf{K}}$ then $R^{a}(h, z)$ is a copy of $h$ written in the alphabet $A(z) \cup A(z)^{-1}$. If all rules of a word $h$ 
are right active for $z$-sectors for some $z \in \overline{\mathbf{K}}$ then $L^{a}\left(h, z_{+}\right)$is a copy of $h$ written in the alphabet $A(z)$.

Lemma 6.5. (i) If $h \equiv h_{1}^{s}$ is a reduced word in $\mathbb{S}, s>1, W$ belongs to the domain of $h$ then $W \cdot h$ and $W$ have the same $\Omega$-coordinates.

(ii) Let $h_{1}$ and $h_{2}$ be two words in $\mathbb{S}$ which are equal in the free group modulo Burnside relations. Suppose that $W$ is in the domain of both $h_{1}$ and $h_{2}$. Then $W \cdot h_{1}=W \cdot h_{2}$.

(iii) Let $h$ be a word in $\mathbb{S}$ containing subword $g$ which is equal to 1 modulo Burnside relations: $h=h_{1} g h_{2}$. Suppose $W$ is in the domain of $h$. Then $W$ is in the domain of $h_{1} h_{2}$ and $W \cdot h_{1} h_{2}=W \cdot h$.

(iv) If $W$ belongs to the domain of $h^{2}$, where $h$ is a cyclically reduced word, then it belongs to the domain of $h^{s}$ for every integer s (in particular, $h^{s}$ has a non-empty domain).

Proof. (i) Recall that non-connecting rules do not change $\Omega$-coordinates and each connecting rule $\mathbf{c}_{\omega}$ replaces $\omega$ by $\omega+1, \omega \in \Omega$. Hence for every word $g$ over $\mathbb{S}$, there exists $\omega$ in $\Omega$ such that the domain of $g$ consists of admissible words with $\Omega$-coordinate $\omega$. Since $W \cdot h_{1}$ is in the domain of $h_{1}$, the $\Omega$-coordinate of $W \cdot h_{1}$ is the same as the $\Omega$-coordinate of $W$. Hence for every $\omega \in \Omega$, the product of occurrences of $\mathbf{c}_{\omega}^{ \pm 1}$ in $h_{1}$ is 1 . The same is true for any power $h=h_{1}^{s}, s \in \mathbb{Z}$, of $h$. Therefore for $W \cdot h$ has the same $\Omega$-coordinate as $W$.

(ii) Let $W$ be in the domain of $h_{1}, h_{2}$. Suppose that $h_{1}$ is equal to $h_{2}$ modulo the Burnside relations in the free group. Clearly it is enough to assume that $W$ has only one sector: $W \equiv z_{1} w z_{2}$. Then by Lemma $6.4(\mathrm{i}) W \cdot h_{i}=z_{1, i}\left(R^{a}\left(h_{i}, z_{1}\right)\right)^{-1} w L^{a}\left(h_{i}, z_{2}\right) z_{2, i}, i=1,2$. Since $R^{a}\left(., z_{1}\right)$ and $L^{a}\left(., z_{2}\right)$ are homomorphisms and $h_{1}$ and $h_{2}$ are equal modulo Burnside relations, $R^{a}\left(h_{1}, z_{1}\right)=$ $R^{a}\left(h_{2}, z_{1}\right)$ and $L^{a}\left(h_{1}, z_{2}\right)=L^{a}\left(h_{2}, z_{2}\right)$ modulo Burnside relations. But all Burnside relations hold in $\langle\mathbf{A}\rangle$, so $\left(R^{a}\left(h_{1}, z_{1}\right)\right)^{-1} w L^{a}\left(h_{1}, z_{2}\right)=\left(R^{a}\left(h_{2}, z_{1}\right)\right)^{-1} w L^{a}\left(h_{2}, z_{2}\right)$.

It remains to show that the $\Omega$-coordinates of $W \cdot h_{1}$ and $W \cdot h_{2}$ are the same. These $\Omega$-coordinates are determined by the projections of $h_{1}$ and $h_{2}$ on the alphabet $\left\{\mathbf{c}_{1}, \ldots, \mathbf{c}_{2 n+1}\right\}$ (because other rules do not change the $\Omega$-coordinate). Each connecting rule $\mathbf{c}_{\omega}$ induces a partial transformation on $\Omega$ of the form $\omega \rightarrow \omega+1$. Therefore every product of $\mathbf{c}_{\omega}, \omega \in \Omega$ which has a non-empty domain considered as a transformation of $\Omega$, must be freely equal to a word of the form $\left(\mathbf{c}_{i} \mathbf{c}_{i+1} \ldots \mathbf{c}_{s}\right)^{ \pm 1}$ for some $i \leq s$.

Since $h_{1}$ and $h_{2}$ are equal modulo Burnside relations, their projections are also equal modulo Burnside relations. But words of the form $\left(\mathbf{c}_{i} \mathbf{c}_{i+1} \ldots \mathbf{c}_{s}\right)^{ \pm 1}$ can be equal modulo Burnside relations only if they are graphically equal. Thus projections of $h_{1}$ and $h_{2}$ onto the alphabet $\left\{\mathbf{c}_{1}, \ldots, \mathbf{c}_{2 n+1}\right\}$ are freely equal. It remains to observe that if two words in $\mathbf{c}_{\omega}, \omega \in \Omega$, are freely equal and induce non-empty transformations of $\Omega$, then they induce the same transformation of $\Omega$. Hence $W \cdot h$ and $W \cdot h^{\prime}$ have the same $\Omega$-coordinates.

(iii) Indeed by part (ii) $W \cdot h_{1}$ is equal to $W \cdot h_{1} g$ and is in the domain of $h_{2}$. Therefore $W$ is in the domain of $h_{1} h_{2}$ and $W \cdot h_{1} h_{2}=W \cdot h$.

(iv) Let $W$ belong to the domain of $h^{2}$ where $h$ is cyclically reduced. It is enough to consider the case when $W$ consists of one sector: $W \equiv z_{1} w z_{2}$.

Suppose first that $h$ does not contain rules which lock $z_{1}$-sectors. By Lemma 6.4(i) $W \cdot h^{2}=$ $z_{1}^{\prime}\left(R^{a}\left(h^{2}, z_{1}\right)\right)^{-1} w L^{a}\left(h^{2}, z_{2}\right) z_{2}^{\prime}$ where $z_{1}^{\prime}$ and $z_{2}^{\prime}$ differ from $z_{1}$ and $z_{2}$ only by the $\Omega$-coordinate. By part (i), $z_{1}^{\prime} \equiv z_{1}$ and $z_{2}^{\prime} \equiv z_{2}$. So $W \cdot h^{2}$ is in the domain of $h$, because $W \cdot h^{2}$ has the same $\Omega$-coordinate as $W$, and $h$ does not contain rules which lock $z_{1}$-sectors. Thus $W$ is in the domain of $h^{s}$ for all positive integers $s$. By part (ii), $W \cdot h^{n}=W$. Therefore $W$ is in the domain of $h^{-2}$ $(n>2)$. This implies as before that $W$ is in the domain of $h^{s}$ for all negative integers $s$. 
Now suppose that $h$ contains a rule $\tau$ which locks $z_{1}$-sectors, $h \equiv h_{1} \tau h_{2}$ for some words $h_{1}, h_{2}$ in $\mathbb{S}$. Then $W \cdot h_{1}$ has the form $z_{1}^{\prime} z_{2}^{\prime}$ where $z_{1}^{\prime}, z_{2}^{\prime}$ differ from $z_{1}, z_{2}$ only by the $\Omega$-coordinate, $h^{2} \equiv h_{1} \tau h_{2} h_{1} \tau h_{2}$. Then $W \cdot h_{1}$ and $W \cdot h_{1} \tau h_{2} h_{1}$ have the same $\Omega$-coordinates because both words are in the domain of $\tau$. Since $\tau$ locks $z_{1}$-sectors, $W \cdot h_{1}$ and $W \cdot h_{1} \tau h_{2} h_{1}$ are both equal to the word of the form $z_{1}^{\prime} z_{2}^{\prime}$ in $H_{k a}^{*}$. Since $W \cdot h_{1}$ is in the domain of $\tau h_{2} h_{1} \tau h_{2}, W \cdot h_{1} \tau h_{2} h_{1}$ must be in the domain of $\tau h_{2} h_{1} \tau h_{2}$ too. Thus $W$ is in the domain of $h_{1} \tau h_{2} h_{1} \tau h_{2} h_{1} \tau h_{2} \equiv h^{3}$. Considering consequently $W \cdot h, W \cdot h^{2}, \ldots$ instead of $W$, we conclude that $W$ is in the domain of $h^{s}$ for every positive integer $s$. Using part (ii), as before, we deduce the same fact for negative $s$.

Lemma 6.6. For every admissible word $U$ with $\Omega$-coordinate $\omega$, and every two non-empty words $h_{1}, h_{2}$ over $\mathbb{S}_{\omega}$ if $W$ is in the domains of $h_{1}, h_{1}=h_{2}$ modulo Burnside relations then $U \cdot h_{1}=U \cdot h_{2}$.

Proof. By Lemma 6.3, we can assume that $U \equiv z u z^{\prime}$ is a one-sector admissible word. Recall that either all rules from $\mathbb{S}_{\omega}$ are left (resp. right) active for $z$-sectors or all these rules lock $z$-sectors or none of these rules are active or locking for $z$-sectors. Hence we can conclude that since $U$ is in the domain for $h_{1}$, and $h_{1}$ is not empty, it is also in the domain for $h_{2}$. The equality $W \cdot h_{1}=W \cdot h_{2}$ follows from Lemma 6.5 (ii).

Words in the alphabet $\mathbb{S}$, that do not have subwords which are equal to 1 modulo Burnside relations, will be called Burnside-reduced.

Lemma 6.7. Let $W \equiv z_{1} w z_{2}$ be an admissible word consisting of one sector, $z_{2} \not \equiv z_{1}^{-1}$. Let $h$ be a word in $\mathbb{S}$. Suppose that $R^{a}\left(\tau, z_{1}\right)$ (resp. $L^{a}\left(\tau, z_{2}\right)$ ) is non-empty for all $\tau$ in $h$. Suppose that either none of the rules in $h$ is from $\mathbb{S}_{2 n+1}$ or all of them are from $\mathbb{S}_{2 n+1}$. Suppose also that $W \cdot h=W$. Finally suppose that $z_{1} \neq \kappa(1), z_{1} \neq \rho(1)^{-1}$. Then $h$ is equal to 1 modulo Burnside relations.

Proof. We only consider the case when $R^{a}\left(\tau, z_{1}\right)$ is not empty because the $L^{a}$-case is similar. Recall that by Lemma 6.4 (ii) then $L^{a}\left(\tau, z_{1}\right)$ is empty for every $\tau$ from $h$. By Lemma 6.4(i), $W \cdot h$ is graphically equal to $z_{1} R^{a}\left(h, z_{1}\right)^{-1} w z_{2}$ and by Lemma 6.4 (iii) $R^{a}\left(h, z_{1}\right)$ is a copy of the word $h$ written in the alphabet $A\left(z_{1}\right) \cup A\left(z_{1}\right)^{-1}$. Since $W \cdot h=W, R^{a}\left(h, z_{1}\right)=1$ in the subgroup $\left\langle A\left(z_{1}\right)\right\rangle$ of $H_{k a}^{*}$. This subgroup is a free Burnside group of exponent $n$ (freely generated by $A\left(z_{1}\right)$ ). Therefore $h$ is equal to 1 modulo Burnside relations.

The presentation of $H_{k a}^{*}$ satisfies conditions (Z1) if we consider letters from $\mathbf{A}$ as 0-letters and let $Y=\mathbf{K}$, so we can apply Lemmas from Section 3.2. In particular, by Lemma 3.3, an admissible word $W$ is $\mathbf{K}$-reduced if and only if it does not have sectors $z u z^{-1}$ where $u=1$ modulo relations of $H_{a}$.

Lemma 6.8. Let $W$ be a $\mathbf{K}$-reduced admissible word which is in the domain of some word $h$ over $\mathbb{S}$. Then $W \cdot h$ is also $\mathbf{K}$-reduced.

Proof. Indeed, one only needs to check that no sector in $W \cdot h$ is equal to 1 in $H_{k a}^{*}$. Indeed, it is trivial for sectors of the form $z w z_{+}$because the application of $\tau$ do not change the $\overline{\mathbf{K}}$-projections of $\mathbf{K}$-letters and the free group $\langle\mathbf{K}\rangle$ is a retract of $H_{k a}^{*}$. If a sector of $W$ has the form $z w z^{-1}$ then the corresponding sector in $W \cdot h$ has the form $z u w u^{-1} z^{-1}$ for some $u$ by Lemma 6.4. This sector is equal to 1 only if $u w u^{-1}=1$. But then $w=1$ and the sector $z w z^{-1}$ is also equal to 1 in $H_{k a}^{*}$.

From now on we consider only $\mathbf{K}$-reduced admissible words. 
Lemma 6.9. Let $W \equiv z w z^{-1}$ be an admissible word consisting of one sector. Let $h$ be a reduced word in $\mathbb{S}$ and $W$ is in the domain of $h$. Then $h$ does not contain rules which lock $z$-sectors.

Proof. Indeed, suppose $h \equiv h_{1} \tau h_{2}$ for some $\tau \in \mathbb{S}$ which locks $z$-sectors. Then by Lemma 6.4

$$
W \cdot h_{1}=z^{\prime} R^{a}\left(h_{1}, z\right) w\left(R^{a}\left(h_{1}, z\right)\right)^{-1}\left(z^{\prime}\right)^{-1}=z^{\prime}\left(z^{\prime}\right)^{-1}
$$

because $W \cdot h_{1}$ is in the domain of $\tau$. This implies that $w=1$ and $W=1$ which contradicts the assumption that $W$ is reduced in $H_{k a}^{*}$.

Lemma 6.10. Let $W \equiv z w z^{-1}$ be an admissible word consisting of one sector. Let $h$ be a word in $\mathbb{S}$ and all rules in $h$ are left active for $z$-sectors. Suppose that $W \cdot h=W$. Then

(i) $R^{a}(h, z)$ commutes with $w$

(ii) if in addition $z \not \equiv \kappa(1)$ and $h$ either does not contain rules from $\mathbb{S}_{2 n+1}$ or contains only rules from $\mathbb{S}_{2 n+1}$ then $h$ is equal modulo Burnside relations to the power of a certain word root $\omega_{\omega}(w)$ depending only on $w$ and the $\Omega$-coordinate of $z$. Moreover $W \cdot \operatorname{root}_{\omega}(w)=W$.

Proof. The first statement immediately follows from Lemma 6.4(i). To prove (ii), recall that by Lemma 6.4(iii) $R^{a}(h, z)$ is a copy of $h$ written in the alphabet $A(z)^{-1}$ and the subgroup $\langle A(z)\rangle$ of $H_{k a}^{*}$ is the free Burnside group of exponent $n$. By Lemma 4.18 then $R^{a}(h, z)$ and $w$ belongs to the same maximal cyclic subgroup $\left\langle w_{0}\right\rangle$ of $\langle A(z)\rangle$ where $w_{0}$ depends only on $w$. Therefore $h$ is equal modulo Burnside relations to a power of a copy of $w_{0}$ written in the alphabet $\mathbb{S}$. Denote this copy by $\operatorname{root}_{\omega}(w)$. Then all rules in $\operatorname{root}_{\omega}(w)$ are left active for $z$-sectors, and so $W$ is in the domain of $\operatorname{root}_{\omega}(w)$. Since $R^{a}\left(\operatorname{root}_{\omega}(w), z\right)=w_{0}$ commutes with $w$, we have $W \cdot \operatorname{root}_{\omega}(w)=W$.

Lemma 6.11. Let $h$ be a reduced word in $\mathbb{S}$ containing a subword of the form $\mathbf{c}_{\omega}^{ \pm 1} h^{\prime} \mathbf{c}_{\omega}^{\mp 1}$ where $h^{\prime}$ is a word in $\mathbb{S}_{\omega}$ or $\mathbb{S}_{\omega+1}$. Let $W \equiv z_{1} w z_{2}$ be an admissible word consisting of one sector, $z_{1} \not \equiv \kappa(1), \rho(1)^{-1}$. Suppose that $\mathbf{c}_{\omega}$ locks $z_{1}$-sectors, but rules from $h^{\prime}$ are (left or right) active for $z_{1}$-sectors. Finally suppose that $W$ is in the domain of $h$. Then $h^{\prime}$ is equal to 1 modulo Burnside relations.

Proof. Indeed, since $\mathbf{c}_{\omega}$ locks $z_{1}$-sectors, by Lemma $6.9 z_{2} \not \equiv z_{1}^{-1}$. Thus $z_{2} \equiv\left(z_{1}\right)_{+}$by the definition of admissible words. From the definition of $\mathbb{S}$, it follows that then either $R^{a}\left(\tau, z_{1}\right)$ is empty for all $\tau \in \mathbb{S}$ or $L^{a}\left(\tau, z_{2}\right)$ is empty for all $\tau \in \mathbb{S}$. Without loss of generality assume that $L^{a}\left(\tau, z_{2}\right)$ is empty for all $\tau$ from $h^{\prime}$. Then $R^{a}\left(\tau, z_{1}\right)$ are not empty for $\tau$ from $h^{\prime}$ and by Lemma 6.4 (iii) $R^{a}\left(h^{\prime}, z_{1}\right)$ is a copy of $h^{\prime}$ written in the alphabet $A\left(z_{1}\right) \cup A\left(z_{1}\right)^{-1}$. By Lemma 6.4 (i), $W \cdot h=z_{1} R^{a}\left(h^{\prime}, z_{1}\right) z_{2}=z_{1} z_{2}$ because $W \cdot h$ is in the domain of $\mathbf{c}_{\omega}^{-1}$ which locks $z_{1}$-sectors. Therefore $R^{a}\left(h^{\prime}, z_{1}\right)=1$, thus $h^{\prime}$ is 1 modulo Burnside relations.

The argument in the proof of Lemma 6.11 can be easily generalized to prove the following statement.

Lemma 6.12. Let $h$ be a reduced word in $\mathbb{S}$ of the form $h_{1} h_{2}$. Let $W \equiv z_{1} w z_{2}$ be an admissible word consisting of one sector, $z_{2} \equiv\left(z_{1}\right)_{+}, z_{1} \not \equiv \kappa(1), \rho(1)^{-1}$. Suppose that $W$ is in the domain of $h$ and $W \cdot h_{1}=W \cdot h_{1} h_{2}$ and that rules from $h_{2}$ are active for $z_{1}$-sectors. Then $h_{2}=1$ modulo Burnside relations. 
Lemma 6.13. Let $W \equiv z u z^{\prime}$ be a one-sector admissible word and $h, g$ be words over $\mathbb{S}, W \cdot h=W$, $W \cdot g h g^{-1}=W$. Suppose that at least one of the following three possibilities holds for all rules $\tau$ of $h, g$ simultaneously:

(i) $R^{a}(\tau, z)$ is not empty;

(ii) $L^{a}\left(\tau, z^{\prime}\right)$ is not empty;

(iii) $L^{a}(\tau, z)$ and $R^{a}(\tau, z)$ are empty.

Suppose also that either all rules from $h, g$ are not from $\mathbb{S}_{2 n+1}$ or all of them are from $\mathbb{S}_{2 n+1}$. Finally suppose that $z \not \equiv \kappa(1), z \not \equiv \rho(1)^{-1}$. Then either $g, h$ commute modulo Burnside relations or $W \cdot g=W$.

Proof. Consider two possible cases.

Case 1. $W \equiv z u z_{+}$. Since $z \not \equiv \kappa(1), \rho(1)^{-1}$, by Lemma 6.4(ii) possibilities (i) and (ii) from the formulation of the lemma cannot both hold. By assumption, we have the following possibilities:

Case 1.1. For every rule $\tau$ from $h, g, R^{a}(\tau, z)$ is not empty but $L^{a}\left(\tau, z_{+}\right)$is empty.

Case 1.2. For every rule $\tau$ from $g, h, L^{a}\left(\tau, z_{+}\right)$is not empty but $R^{a}(\tau, z)$ is empty.

Case 1.3. For every rule $\tau$ from $g, h R^{a}(\tau, z)$ and $L^{a}\left(\tau, z_{+}\right)$are empty.

In Cases 1.1, 1.2, by Lemma 6.7, $h=1$ modulo Burnside relations, so $g, h$ commute modulo Burnside relations, as required.

In Case 1.3 by Lemma 6.4(i) for every $\tau$ in $g$, we have $W \cdot \tau=W$, and so $W \cdot g=W$, as required.

Case 2. $W \equiv z u z^{-1}$ (again $z \not \equiv \kappa(1), \rho(1)^{-1}$ by assumption).

Case 2.1. Suppose first that for every rule from $g, h, R^{a}(\tau, z)$ is not empty.

In this case by Lemma $6.10 h$ commutes with $g h g^{-1}$ modulo Burnside relations which implies by Lemma 4.19 that $h$ commutes with $g$ modulo Burnside relations.

Case 2.2. Suppose now that every rule $\tau$ of $g, h, R^{a}(\tau, z)$ (and hence $L^{a}\left(\tau, z^{-1}\right)$ ) is empty.

In this case again by Lemma 6.4(i), for every $\tau \in \mathbb{S}_{\omega}$ we have $W \cdot \tau=W$, and so $W \cdot g=W$, as required.

\subsection{Stabilizers in $P(\mathbb{S})$ of subwords of the hub}

For every $i=1, \ldots, N$ let $\Lambda_{i}$ be the subword of $\Lambda$ starting with $\lambda(i)$ and ending with $\lambda(i+1)$ (" $N+1$ " here is 1).

Suppose $i>1$, and that $\Lambda_{i}(0)$ is in the domain of a word $h$ over $\mathbb{S}$. Let $U_{i}(\omega) \equiv \Lambda_{i}(0) \cdot h$, $U_{i} \equiv \lambda(i) u_{1} \kappa(i) \rho(i) u_{2} \ldots \overleftarrow{\kappa}(i, n) u_{2 n+2} \lambda(i+1)$ for some words $u_{1}, \ldots, u_{2 n+2}$ (notice that the word between $\kappa(i)$ and $\rho(i)$ is empty, $i=2, \ldots, N$ because every rule in $\mathbb{S}$ locks $\kappa(i)$-sectors).

Let $U_{j}$ be the admissible word obtained from $U_{i}$ by replacing all indexes $i$ in $\overline{\mathbf{K}}$-letters by $j$ :

$$
U_{j} \equiv \lambda(j) u_{1} \kappa(j) \rho(j) u_{2} \ldots \overleftarrow{\kappa}(j, n) u_{2 n+2} \lambda(j+1)
$$

It is easy to see by inspection that rules of $\mathbb{S}$ act in the same way on $U_{j}$ for all $j>1$. Therefore, an induction on $|h|$ gives us

$$
\Lambda_{j}(0) \cdot h=U_{j}(\omega)
$$

Let us also define $U_{1}$ : 


$$
U_{1} \equiv \lambda(1) u_{1} \kappa(1) \Phi\left(u_{1}\right) \rho(1) u_{2} \ldots \overleftarrow{\kappa}(1, n) u_{2 n+2} \lambda(2)
$$

where $\Phi$ is the homomorphism from $\langle A(\lambda(1))\rangle$ to $\langle A(\kappa(1))\rangle$ which takes every letter $\overline{\tau(\omega, a)}$ to $a(\kappa(1))^{-1}$ if $\omega$ is even and greater than 0 , and every other letter to 1 . Notice that the map $\Phi$ from $A(\lambda(1))$ to $A(\kappa(1)) \cup\{1\}$ is indeed extendable to a homomorphism from $\langle A(\lambda(1))\rangle$ to $\langle A(\kappa(1))\rangle$ because $\langle A(\lambda(1))\rangle$ is a free Burnside group and $\langle A(\kappa(1))\rangle$ satisfies all Burnside relations.

Remark 6.14. Notice that $U_{1}$ would be a copy of $U_{j}$ obtained by replacing index 1 for $j$ if not for the $\kappa$-sector: the $\kappa(j)$-sector of an admissible word contains no A-letters, but $\kappa(1)$-sectors may contain A-letters. If the $\mathbf{A}$-subword of the $\kappa(1)$-sector of $U_{1}$ is trivial (say, if $U_{1}$ is in the domain of $\mathbf{c}_{2 n}^{ \pm 1}$ ), then $U_{1}$ is a copy of $U_{j}$.

For every word $u \equiv a_{i_{1}} \ldots a_{i_{s}}$ over $\mathcal{A} \cup \mathcal{A}^{-1}$ and every even $\omega$ from $\Omega$ let $T_{\omega}(u)$ be the word

$$
\tau\left(\omega, a_{i_{1}}\right) \ldots \tau\left(\omega, a_{i_{s}}\right)
$$

For every odd $\omega \neq 2 n+1$ from $\Omega$ let

$$
T_{\omega}(u) \equiv \tau\left(\omega, a_{i_{s}}\right) \ldots \tau\left(\omega, a_{i_{1}}\right) .
$$

Let $z, z^{\prime}$ be two letters from $\overline{\mathbf{K}}, z^{\prime} \equiv z_{+}$or $z^{\prime} \equiv z^{-1}$ then every sector of an admissible word of the form $\left(z(\omega) u z^{\prime}(\omega)\right)^{ \pm 1}$ will be called a sector of the type (form) $\left[z z^{\prime}\right]$.

Lemma 6.15. Let $j>1$. Suppose $\Lambda_{j}(0)$ is in the domain of a word of the form $h \mathbf{c}_{2 n}$ where the word $h$ is Burnside-reduced and does not contain $\mathbf{c}_{2 n}^{ \pm 1}$. Let $U_{j}(2 n+1) \equiv \Lambda_{j}(0) \cdot h \mathbf{c}_{2 n}$. Then $\Lambda_{1}(0)$ is in the domain of $h \mathbf{c}_{2 n}$ and $U_{1}(2 n+1) \equiv \Lambda_{1}(0) \cdot h \mathbf{c}_{2 n}$.

Proof. Suppose that $h$ contains a subword of the form $\mathbf{c}_{i} h^{\prime} \mathbf{c}_{i}^{-1}$ where $h^{\prime}$ contains no connecting rules: $h \equiv h_{1} \mathbf{c}_{i} h^{\prime} \mathbf{c}_{i}^{-1} h_{2}$ for some even $i$ from 0 to $2 n-2$. Then consider the sector $W^{\prime}$ of the type $[\overleftarrow{\kappa}(j, n) \lambda(j+1)]$ of $\Lambda_{j}(0) \cdot h_{1} \mathbf{c}_{i}$. Since $\mathbf{c}_{i}$ locks $\overleftarrow{\kappa}(j, n)$-sectors, $W^{\prime}$ has the form $\overleftarrow{\kappa}\left(j, n, \omega^{\prime}\right) \lambda\left(j+1, \omega^{\prime}\right)$ (the A-part of the sectors is trivial). Since $W^{\prime} \cdot h^{\prime}$ is in the domain of $\mathbf{c}_{i}^{-1}, W^{\prime}=W^{\prime} \cdot h^{\prime}$. Since all rules in $h^{\prime}$ are left active for $\overleftarrow{\kappa}(j, n)$-sectors (all these rules belong to $\mathbb{S}_{i+1}$, and $i$ is odd), by Lemma $6.11, h^{\prime}=1$ modulo Burnside relations, a contradiction. Similarly, $h$ does not contain subwords of the form $\mathbf{c}_{i}^{-1} h^{\prime} \mathbf{c}_{i}$ with even $i>0$ where $h^{\prime}$ does not contain connecting rules.

Suppose that $h$ contains a subword of the form $\mathbf{c}_{i} h^{\prime} \mathbf{c}_{i}^{-1}$ where $h^{\prime}$ contains no connecting rules and $i$ is odd, $i \neq 2 n-1$. Then applying the argument similar to the one used in the previous paragraph to the sector of the form $[\vec{\kappa}(j, n) \overleftarrow{\kappa}(j, n)]$, we get a contradiction. Similarly one can prove that $h$ does not contain subwords of the form $\mathbf{c}_{i}^{-1} h^{\prime} \mathbf{c}_{i}$ where $h^{\prime}$ contains no connecting rules, $i$ is any odd number between 1 and $2 n-1$.

Suppose now that $h$ contains one of the connecting rules twice. Since $\Lambda_{j}(0)$ has $\Omega$-coordinate 0 , and $\Lambda_{j}(0) \cdot h$ has $\Omega$-coordinate $2 n$, the facts proved in the previous two paragraphs imply that $h$ contains a subword of the form

$$
h_{0} \mathbf{c}_{0} h_{1} \ldots h_{2 n-2} \mathbf{c}_{2 n-1} h_{2 n-1} \mathbf{c}_{2 n-1}^{-1} h_{2 n-2}^{\prime} \ldots \mathbf{c}_{0}^{-1} h_{0}^{\prime}
$$

where the subwords $h_{\omega}, h_{\omega}^{\prime}$ are words over $\mathbb{S}_{\omega}$. Let then $W^{\prime}$ be the sector of the form $[\rho(j) \vec{\kappa}(j, 1)]$ of $\Lambda_{j}(0) \cdot h_{0}$. The word $W^{\prime}$ is in the domain of

$$
h^{\prime \prime} \equiv \mathbf{c}_{0} h_{1} \ldots h_{2 n-2} \mathbf{c}_{2 n-1} h_{2 n-1} \mathbf{c}_{2 n-1}^{-1} h_{2 n-2}^{\prime} \ldots \mathbf{c}_{0}^{-1},
$$


$\mathbf{c}_{0}$ locks $W^{\prime}$, all other rules from $h^{\prime \prime}$ are right active for $\rho(j)$-sectors. None of these rules belong to $\mathbb{S}_{2 n+1}$. Hence by Lemma $6.11, h^{\prime}=1$ modulo Burnside relations, a contradiction. Therefore $h$ contains each of its connecting rules only once.

Hence $h$ can be represented in the form $h_{0} \mathbf{c}_{0} h_{1} \mathbf{c}_{1} \ldots h_{2 n}$ for some words $h_{i}$ over $\mathbb{S}_{i}, i=0, \ldots, 2 n$.

For some words $u_{0}, \ldots, u_{2 n}$ over $\mathcal{A} \cup \mathcal{A}^{-1}, h_{i} \equiv T_{i}\left(u_{i}\right), i=0, \ldots, 2 n$. Let us prove by induction on $i$ that $u_{i}=u_{0}$ modulo Burnside relations. Indeed, this statement is trivial for $i=0$. Suppose that it is true for some $\omega$ from 0 to $2 n-1$. Let us prove it for $\omega+1$. Since $u_{0}=u_{1}=\ldots=u_{\omega}$ modulo Burnside relations, by Lemma 6.6,

$$
\Lambda_{j}(0) \cdot h_{0} \mathbf{c}_{0} h_{1} \mathbf{c}_{1} \ldots h_{\omega} \mathbf{c}_{\omega}=\Lambda_{j}(0) \cdot T_{0}(u) \mathbf{c}_{0} T_{1}(u) \mathbf{c}_{1} \ldots T_{\omega}(u) \mathbf{c}_{\omega}=V .
$$

In particular the $\Omega$-coordinate of $V$ is $\omega+1$.

Suppose that $\omega$ is even. Then as in the proof of Lemma 5.2 the sector of the form $[\vec{\kappa}(j, n) \overleftarrow{\kappa}(j, n)]$ of $V$ is equal to $\vec{\kappa}(j, n, \omega+1) u \overleftarrow{\kappa}(j, n, \omega+1)$. Since $V \cdot T_{\omega+1}\left(u_{\omega+1}\right)$ is in the domain of $\mathbf{c}_{\omega+1}$ and $\mathbf{c}_{\omega+1}$ locks $\vec{\kappa}(j, n)$-sectors, we get that the sector of the form $[\vec{\kappa}(j, n) \overleftarrow{\kappa}(j, n)]$ of $V \cdot T_{\omega+1}\left(u_{\omega+1}\right)$ is equal to $\vec{\kappa}(j, n, \omega+1) \overleftarrow{\kappa}(j, n, \omega+1)$. By Lemma 6.4 , we get that $u_{\omega+1}=u$ modulo Burnside relations, as required. $V$.

If $\omega$ is odd then we can apply the above argument to the sector of the form $[\overleftarrow{\kappa}(j, n) \lambda(j+1)]$ of

Therefore we can apply Lemma $6.62 n+1$ times and conclude that $\Lambda_{1}(0) \cdot h_{0}=\Lambda_{1}(0) \cdot T_{0}(u)$, $\Lambda_{1}(0) \cdot h_{0}$ is in the domain of $\mathbf{c}_{0} h_{1}$ and $\Lambda_{1}(0) \cdot h_{0} \mathbf{c}_{0} h_{1}=\Lambda_{1}(0) \cdot T_{0}(u) \mathbf{c}_{0} T_{1}(u), \ldots, \Lambda_{1}(0) \cdot h_{1} \mathbf{c}_{0} \ldots h_{2 n-1}=$ $\Lambda_{1}(0) \cdot T_{0}(u) \mathbf{c}_{0} \ldots T_{2 n-1}(u)$ is in the domain of $\mathbf{c}_{2 n-1} h_{2 n}$ and

$$
\Lambda_{1}(0) \cdot h_{1} \mathbf{c}_{0} \ldots h_{2 n-1} \mathbf{c}_{2 n-1} h_{2 n}=\Lambda_{1}(0) \cdot T_{0}(u) \mathbf{c}_{0} \ldots T_{2 n-1}(u) \mathbf{c}_{2 n-1} T_{2 n}(u)=V^{\prime} .
$$

All sectors of the latter word contain no A-letters except for the $\lambda(1)$-sector, $\kappa(1)$-sector, and $\rho(1)$ sector. The first and the third of these sectors contain copies $\overline{h \mathbf{c}_{2 n}}$ of the word $h \mathbf{c}_{2 n}$, the $\kappa(1)$-sector contains $u^{n}$ (as in the proof of Lemma 5.2) which is equal to 1 modulo relations of $\langle A(\kappa(1))\rangle$. Clearly $\Phi\left(\overline{h \mathbf{c}_{2 n}}\right)=u^{n}$. Thus $\Lambda_{1}(0) \cdot h \mathbf{c}_{2 n}$ exists and is equal to $U_{1}(2 n+1)$.

Proposition 6.16. Suppose that for some Burnside-reduced word $h$ over $\mathbb{S}$, and some $j>1$, $\Lambda_{j}(0) \cdot h=\Lambda_{j}(0)$. Then for every $i=1, \ldots, N, \Lambda_{i}(0) \cdot h=\Lambda_{i}(0)$.

Proof. If $h$ is empty, the statement is obviously true. By (20), the statement is also true if $i>1$. So let $h$ be non-empty and $i=1$.

Then as in the proof of Lemma 6.15, Lemma 6.11 implies that $h$ contains no subwords of the form $\mathbf{c}_{i}^{ \pm 1} f \mathbf{c}_{i}^{\mp 1}$ where $f$ does not contain connecting rules, $i \neq 0,2 n$. Hence $h$ can be represented in the form

$$
h_{1} \mathbf{c}_{2 n} g_{1} \mathbf{c}_{2 n}^{-1} h_{2}^{-1} \mathbf{c}_{0}^{-1} h_{2}^{\prime} \mathbf{c}_{2 n} g_{2} \mathbf{c}_{2 n}^{-1} h_{3}^{-1} \mathbf{c}_{0}^{-1} h_{3}^{\prime} \mathbf{c}_{2 n} g_{3} \ldots g_{s-1} \mathbf{c}_{2 n}^{-1} h_{s}^{-1}
$$

where $g_{p}$ are non-empty words over $\mathbb{S}_{2 n+1}, p=1, \ldots, s-1, h_{1}, \ldots, h_{s}, h_{2}^{\prime}, \ldots, h_{s-1}^{\prime}$.

We claim that for every $t=2, \ldots, s-1$, the word

$$
V \equiv \Lambda_{j}(0) \cdot h_{1} \mathbf{c}_{2 n} g_{1} \mathbf{c}_{2 n}^{-1} h_{2}^{-1} \mathbf{c}_{0}^{-1} h_{2}^{\prime} \mathbf{c}_{2 n} g_{2} \mathbf{c}_{2 n}^{-1} \ldots g_{t-1} \mathbf{c}_{2 n}^{-1} h_{t}^{-1} \mathbf{c}_{0}^{-1}
$$

is equal to $\Lambda_{j}(0) \cdot T_{0}\left(u_{t}\right)$ for some word $u_{t}$ over $\mathbf{A} \cup \mathbf{A}^{-1}$.

Indeed, let

$$
V^{\prime} \equiv \Lambda_{j}(0) \cdot h_{1} \mathbf{c}_{2 n} g_{1} \mathbf{c}_{2 n}^{-1} h_{2}^{-1} \mathbf{c}_{0}^{-1} h_{2}^{\prime} \mathbf{c}_{2 n} g_{2} \mathbf{c}_{2 n}^{-1} \ldots g_{t-1} \mathbf{c}_{2 n}^{-1} .
$$


Then $V \equiv V^{\prime} \cdot h_{t}^{-1} \mathbf{c}_{0}^{-1}$. By Lemma 6.11 , the word $h_{t}$ is represented in the form $p_{1} \mathbf{c}_{1} p_{2} \mathbf{c}_{2} \ldots p_{2 n}$ where $p_{i} \equiv T_{i}\left(v_{i}\right)$ for some words $v_{i}$ over $\mathbb{S}_{i}, i=1, \ldots, 2 n$. Since $V^{\prime} \cdot h_{t}^{-1}$ is in the domain of $\mathbf{c}_{0}^{-1}$, $V^{\prime} \cdot h_{t}^{-1}$ has the form

$$
\lambda(j, 1) \kappa(j, 1) \rho(j, 1) \vec{\kappa}(j, 1,1) w_{1} \overleftarrow{\kappa}(j, 1,1) \vec{\kappa}(j, 2,1) w_{2} \ldots \vec{\kappa}(j, n, 1) w_{n} \overleftarrow{\kappa}(j, n, 1) \lambda(j+1,1) .
$$

Since $V^{\prime} \cdot h_{t}^{-1} T_{1}\left(v_{1}\right)$ is in the domain of $\mathbf{c}_{1}$, and $\mathbf{c}_{1}$ locks any $\vec{\kappa}(j, i)$-sector, by Lemma 6.4(iii), each of the words $w_{1}, \ldots, w_{n}$ is a copy of $v_{1}$. Hence $V^{\prime} \cdot h_{t}^{-1} \mathbf{c}_{0}^{-1}=\Lambda_{j}(0) \cdot T_{0}\left(v_{1}\right)$ as required.

Therefore $\Lambda_{j}(0)$ is in the domain of $T_{0}\left(u_{t}\right) \mathbf{c}_{0} h_{t} \mathbf{c}_{2 n}$ and $T_{0}\left(u_{t}\right) h_{t}^{\prime} \mathbf{c}_{2 n}$.

Let $U_{j, t}(2 n+1) \equiv \Lambda_{j}(0) \cdot T_{0}\left(u_{t}\right) \mathbf{c}_{0} h_{t} \mathbf{c}_{2 n}, U_{j, t}^{\prime}(2 n+1) \equiv \Lambda_{j}(0) \cdot T_{0}\left(u_{t}\right) h_{t}^{\prime} \mathbf{c}_{2 n}$. By Lemma 6.15, $\Lambda_{1}(0)$ is in the domain of $T_{0}\left(u_{t}\right) \mathbf{c}_{0} h_{t} \mathbf{c}_{2 n}$ and $T_{0}\left(u_{t}\right) h_{t}^{\prime} \mathbf{c}_{2 n}$, and $U_{1, t}(2 n+1)=\Lambda_{j}(0) \cdot T_{0}\left(u_{t}\right) \mathbf{c}_{0} h_{t} \mathbf{c}_{2 n}$, $U_{1, t}^{\prime}(2 n+1)=\Lambda_{j}(0) \cdot T_{0}\left(u_{t}\right) h_{t}^{\prime} \mathbf{c}_{2 n}$.

By Remark 6.14, $U_{1, t}(2 n+1)$ is a copy of $U_{j, t}(2 n+1)$ and $U_{1, t}^{\prime}(2 n+1)$ is a copy of $U_{j, t}^{\prime}(2 n+1)$ An easy induction on the length of a word $g$ over $\mathbb{S}_{2 n+1}$ gives that $U_{1, t}(2 n+1) \cdot g\left(\right.$ resp. $\left.U_{1, t}^{\prime}(2 n+1) \cdot g\right)$ is a copy of $U_{j, t}(2 n+1)$ (resp. $\left.U_{j, t}^{\prime}(2 n+1)\right)$.

Thus we can conclude that

$$
\begin{aligned}
& \left.\left.\left(\ldots\left(\left(\Lambda_{1}(0) \cdot h_{1} \mathbf{c}_{2 n} g_{1}\right) \cdot\left(\mathbf{c}_{2 n}^{-1} h_{2}^{-1} \mathbf{c}_{0}^{-1} T_{0}\left(u_{1}\right)^{-1}\right)\right) \cdot T_{0}\left(u_{1}\right) h_{2}^{\prime} \mathbf{c}_{2 n} g_{2}\right) \cdot \ldots\right) \cdot T_{0}\left(u_{s-1}\right) h_{s-1}^{\prime} \mathbf{c}_{2 n} g_{s-1}\right) . \\
& \mathbf{c}_{2 n}^{-1} h_{s}^{-1}=\Lambda_{1}(0) .
\end{aligned}
$$

By Lemma 6.2 , we obtain $\Lambda_{1}(0) \cdot h=\Lambda_{1}(0)$ as required.

\subsection{Stabilizers of arbitrary admissible words}

We call an admissible word $W$ accepted if for some word $h$ over $\mathbb{S}$, the word $W \cdot h$ is a cyclic shift of $\Lambda(0)^{ \pm 1}$. Clearly $\Lambda(0)$ is an accepted word and every admissible word of the form $\Lambda(0) \cdot h$ is accepted.

In the rest of the section, we are going to prove the following statement.

Proposition 6.17. Let $W$ be an admissible word of the form $z_{1} w_{1} z_{2} \ldots z_{s} w_{s} z_{1}$ (the first and the last $\mathbf{K}$-letters are the same) which does not contain an accepted subword.

(1) Suppose that for some $i=1, \ldots, N$, every sector of $W$ is of one of the types $\left[\kappa(i)^{-1} \kappa(i)\right]$, $[\kappa(i) \rho(i)]$ or $\left[\rho(i) \rho(i)^{-1}\right]$. Let $h, g, b$ be words in the alphabet $\mathbb{S}$ such that $W \cdot h=W, W \cdot g h g^{-1}=W$, $W \cdot b=W$ and $b$ is equal to $g^{-1} h g$ modulo Burnside relations. Then for every integer $s$ there exists a word $b_{s}$ which is equal to $g^{s} h^{-s}$ modulo Burnside relations and such that $W \cdot b_{s}=W$.

(2) Suppose that there is no $i$ from 1 to $N$ such that every sector of $W$ is of one of the types $\left[\kappa(i)^{-1} \kappa(i)\right],[\kappa(i) \rho(i)]$ or $\left[\rho(i) \rho(i)^{-1}\right]$. Let $h, g$ be words in the alphabet $\mathbb{S}$ such that $W \cdot h=W$, $W \cdot g h g^{-1}=W$. Then $W \cdot g=W$ or $g$ and $h$ commute modulo Burnside relations.

Remark 6.18. Clearly if $W \cdot g=W$ or $g$ and $h$ commute modulo Burnside relations then the conclusion of (1) holds, so the conclusion of (2) is stronger than the conclusion of (1).

Let us prove condition (1) of the proposition. Thus consider the case when for some $i=1, \ldots, N$, $W$ contains sectors only of the form $\left[\kappa(i)^{-1} \kappa(i)\right],[\kappa(i) \rho(i)]$ or $\left[\rho(i) \rho(i)^{-1}\right]$.

Notice that since $W$ starts and ends with the same $\mathbf{K}$-letter, the definition of admissible words forces $W$ to have all three of these types of sectors. Indeed, suppose that $W$ contains a sector of the type $[\kappa(i) \rho(i)]$. Then $W$ must contain sectors of the types $[z \kappa(i)]$ and $\left[\rho(i) z^{\prime}\right]$ for some $z, z^{\prime}$. 
By assumption, each of these sectors can be of one of the three types $\left[\kappa(i)^{-1} \kappa(i)\right],[\kappa(i) \rho(i)]$ or $\left[\rho(i) \rho(i)^{-1}\right]$. Hence $z \equiv \kappa(i)^{-1}, z^{\prime} \equiv \rho(i)^{-1}$. Thus sectors of all three types are inside $W$. If $W$ contains a sector of the type $\left[\kappa(i)^{-1} \kappa(i)\right]$ then it must contain a sector of the type $[\kappa(i) z]$ for some $z$ and by assumption $z$ cannot be anything but $\rho(i)$. So $W$ contains a sector of the type $[\kappa(i) \rho(i)]$ which implies that $W$ contains all three types of sectors. Finally if $W$ contains a sector of the type $\left[\rho(i) \rho(i)^{-1}\right]$ then $W$ contains a sector of the type $[z \rho(i)]$ for some $z$. This $z$ must be equal to $\kappa(i)$, so $W$ contains a sector of the type $[\kappa(i) \rho(i)]$ which, as before, implies that $W$ contains all three types of sectors.

Therefore by Lemma $6.9 \mathrm{~h}$ and $g$ do not contain rules from $\mathbb{S}_{0} \cup\left\{\mathbf{c}_{0}, \mathbf{c}_{0}^{-1}\right\}$. We will use this below several times.

We are going to use lemmas from Section 4.3. Consider the free group freely generated by $\mathbb{S} \backslash\left(\mathbb{S}_{0} \cup\left\{\mathbf{c}_{0}, \mathbf{c}_{0}^{-1}\right\}\right)$ as the free product of the group $Q$ freely generated by $\mathbb{S}_{2 n+1} \cup\left\{\mathbf{c}_{2 n}\right\}$ and the group $P$ freely generated by other generators. Consider the homomorphism $v$ from $P$ to $\langle A(\kappa(1))\rangle$ which takes every rule $\tau$ from $P$ to $R^{a}(\tau, \kappa(1))$. This allows us to talk about syllables of words in $P * Q$ and about $v$-trivial elements of $P * Q$.

Notice that if $h$ and the reduced form of $g h g^{-1}$ consist of rules from $P$, we can apply Lemma 6.13 to a sector of the form $\left[\kappa(i)^{-1} \kappa(i)\right]$ (case (i) of the formulation of that lemma holds) and conclude that $g, h$ commute modulo Burnside relations. This implies part (1) of the proposition.

Thus we can assume that $h$ or the reduced form of $g h g^{-1}$ contain rules from $\mathbb{S}_{2 n+1} \cup\left\{\mathbf{c}_{2 n}, \mathbf{c}_{2 n}^{-1}\right\}$. Since $W \cdot h=W, W \cdot g h g^{-1}=W, g$ or the reduced form of $g h g^{-1}$ must contain a rule from $\mathbb{S}_{2 n+1} \cup\left\{\mathbf{c}_{2 n}, \mathbf{c}_{2 n}^{-1}\right\}$.

Therefore there exists a word $x$ in $\mathbb{S}$ such that $W^{\prime}=W \cdot x$ is in the domain of a rule from $\mathbb{S}_{2 n+1} \cup\left\{\mathbf{c}_{2 n}, \mathbf{c}_{2 n}^{-1}\right\}$. By Lemma $6.2 W^{\prime} \cdot x^{-1} h x=W^{\prime}, W^{\prime} \cdot\left(x^{-1} g x\right)\left(x^{-1} h x\right)\left(x^{-1} g x\right)=W^{\prime}, W^{\prime} \cdot x^{-1} b x=$ $W^{\prime}$. It is clear that if for every integer $s$ there exists $b_{s}^{\prime}$ which is equal to $\left(x^{-1} g x\right)^{s}\left(x^{-1} h x\right)\left(x^{-1} g x\right)^{-s}$ modulo Burnside relations and such that, $W^{\prime} \cdot b_{s}^{\prime}=W^{\prime}$, then condition (1) holds for $W, g, h$. Hence we can assume that $W=W^{\prime}$.

Thus all sectors of the form $[\kappa(i) \rho(i)]$ in $W$ are equal to $\kappa(i) \rho(i)$ (since every rule from $\mathbb{S}_{2 n+1} \cup$ $\left\{\mathbf{c}_{2 n}, \mathbf{c}_{2 n}^{-1}\right\}$ locks $\kappa(i)$-sectors).

For every $i=1, \ldots, N$, consider the homomorphism $\vartheta_{i}: \tau \rightarrow L^{a}(\tau, \kappa(i))$ from $P * Q$ to $\langle A(\lambda(i))\rangle$.

Lemma 6.19. Let $x \in P * Q$. Then the following conditions hold:

(i) $\kappa(1) \rho(1) \cdot x=\kappa(1) \rho(1)$ if and only if $x$ is v-trivial.

(ii) $\kappa(1) \rho(1)$ is in the domain of $x$ if and only if every P-syllable of $x$ except possibly the last one has trivial $v$-image.

(iii) Let $W^{\prime} \equiv \kappa(i)^{-1} u \kappa(i)$ be a 1-sector admissible word, $i=1, \ldots, N$. Then $W^{\prime} \cdot x=W^{\prime}$ if and only if $\vartheta_{i}(x)$ commutes with $u$ modulo Burnside relations.

(iv) Every 1-sector admissible word of the form $\left[\kappa(i)^{-1} \kappa(i)\right]$ or $\left[\rho(i) \rho(i)^{-1}\right], i=1, \ldots, N$, is in the domain of $x$.

(v) For every sector $W^{\prime}$ of the form $\left[\rho(i) \rho(i)^{-1}\right]$ of the word $W, W^{\prime} \cdot x=W^{\prime}$.

(vi) If $i=1$ then the word $W$ is in the domain of $x$ if and only if all P-syllables of $x$ except possibly the last one are $v$-trivial. If $i>1$ then the word $W$ is in the domain of $x$.

(vii) If $i=1$ then $W \cdot x=W$ if and only if $x$ is $v$-trivial, and for every sector $W^{\prime} \equiv \kappa(1)^{-1} u \kappa(1)$ of $W, \vartheta_{1}(x)$ commutes with $u$ modulo Burnside relations. If $i>1$ then $W \cdot x=W$ if and only if for every sector $W^{\prime} \equiv \kappa(i)^{-1} u \kappa(i), \vartheta_{i}(x)$ commutes with u modulo Burnside relations. 
Proof. Since we identify letters $\kappa(i, \omega), \omega \in \Omega$, with $\kappa(i)$ and $\rho(i, \omega), \omega \in \Omega$, with $\rho(i)(i=1, \ldots, N)$, all rules from $P$ are applicable to $W$ and to any word of the form $W \cdot x$. Rules from $Q$ are applicable to such a word if and only if every sector of the form $[\kappa(1) \rho(1)]$ is equal to $\kappa(1) \rho(1)$ in $H_{k a}^{*}$. This implies parts (i), (ii) of the lemma.

All rules from $P * Q$ are applicable to any sector of the form $\left[\kappa(i)^{-1} \kappa(i)\right]$ or $\left[\rho(i) \rho(i)^{-1}\right]$. For every rule $\tau$ from $P * Q, R^{a}(\tau, \rho(i))=L^{a}\left(\tau, \rho(i)_{+}\right)=1$. This implies parts (iii),(iv) and (v) because of Lemma 6.4.

Parts (vi), (vii) follow from the previous parts of the lemma by Lemma 6.3.

Lemma 6.20. Let $U \equiv \kappa(i)^{-1} u \kappa(i)$ be a one-sector admissible word $i \geq 1$. Suppose that $U \cdot h=$ $U, U \cdot g h g^{-1}=U$. Then for every positive integer $s, U \cdot g^{s} h g^{-s}=U$.

Proof. By Lemma 6.19 (iii) $\vartheta_{i}(h), \vartheta_{i}\left(g h g^{-1}\right)$ commutes with $u$ modulo Burnside relations. Since $u \neq 1$ modulo Burnside relations (we have agreed to consider only reduced admissible words), by Lemma 4.19, then $\vartheta_{i}(g)$ commutes with $\vartheta_{i}(h)$ modulo Burnside relations. Hence $\vartheta\left(g^{s} h g^{-s}\right)=\vartheta(h)$ modulo Burnside relations. Since by Lemma 6.19 (iv) $U$ is in the domain of $g^{s} h g^{-s}$, by Lemma 6.5(ii), we get $U \cdot g^{s} h g^{-s}=U \cdot h=U$.

Now we are ready to prove part (1) of the proposition if $i>1$.

Lemma 6.21. Part (1) of the Proposition 6.17 holds if $i>1$.

Proof. Indeed all rules from $\mathbb{S}$ lock sectors of the form $[\kappa(i) \rho(i)]$ and are not left active for $\rho(i)$ sectors and for $\rho(i)^{-1}$-sectors. Therefore if $W^{\prime}$ is any of the sectors of $W$ of the form $[\kappa(i) \rho(i)]$ or $\left[\rho(i) \rho(i)^{-1}\right]$ then $W^{\prime} \cdot g=W^{\prime}$, so for every integer $s, W^{\prime} \cdot g^{s} h g^{-s}=W^{\prime}$. By Lemma 6.20 the same conclusion can be drawn for sectors of the form $\left[\kappa(i)^{-1} \kappa(i)\right]$. Hence by Lemma $6.3 \mathrm{~W} \cdot g^{s} h g^{-s}=W$ as required.

Now let $i=1$. It is obvious that we can assume that $g, h \neq 1$ modulo Burnside relations.

Lemma 6.22. If there exists a word $x \in P * Q$ such that $g=x p x^{-1}, h=x q x^{-1}$ modulo Burnside relations, where $p, q \in P$, then $g$ and $h$ commute modulo Burnside relations.

Proof. Suppose that such a word $x$ exists. By Lemma 6.19 (i), the word $h$, the reduced form of $g h g^{-1}$ and the word $b$ from the formulation of part (1) of the proposition are $v$-trivial. By Lemma 4.21, $g$ is also $v$-trivial. Then by Lemma 4.20(iii) (since $g, h \neq 1$ ) there exist words $x_{1}$ and $x_{2}$ such that $x_{1} p x_{1}^{-1}=g, x_{2} q x_{2}^{-1}=h$ modulo Burnside relations, $x_{1} p x_{1}^{-1}, x_{2} q x_{2}^{-1}$ are $v$-trivial. Since $h, g \neq 1$ modulo Burnside relations, $x_{1} p x_{1}^{-1}, x_{2} q x_{2}^{-1}$ can be considered reduced. This implies that $v(p)=v(q)=1$.

We have that $x^{-1} x_{1}$ commutes with $p$ modulo Burnside relations. Therefore $x^{-1} x_{1}$ belongs to the centralizer of $p$ modulo Burnside relations. The centralizer of every non-trivial element of a free Burnside group is cyclic of order $n$ by Lemma 4.18. Therefore the centralizer of $p$ in the free Burnside factor of $P * Q$ and in the free Burnside factor of $P$ are the same. Thus $x^{-1} x_{1}$ belongs to $P$ modulo Burnside relations and $x=x_{1} p_{1}$ for some $p_{1} \in P$ from the same cyclic subgroup as $p$. Since $x_{1} p x_{1}^{-1}$ is $*$-reduced and $v$-trivial, all $P$-syllables of $x_{1} p_{1}$ except for, possibly, the last one are $v$-trivial. Hence by Lemma $6.19(\mathrm{vi}), W$ is in the domain of $x_{1} p_{1}$. 
Notice that $x_{1} p_{1} q p_{1}^{-1} x_{1}^{-1}=x q x^{-1}=h$ modulo Burnside relations. Hence by Lemma 6.5(ii) $W \cdot x_{1} p_{1} q p_{1}^{-1} x_{1}^{-1}=W \cdot h=W$. The word $g h g^{-1}$ is equal to

$$
x_{1} p x_{1}^{-1} x_{1} p_{1} q p_{1}^{-1} x_{1}^{-1} x_{1} p^{-1} x_{1}^{-1}=x_{1} p p_{1} q p_{1}^{-1} p^{-1} x_{1}^{-1}
$$

modulo Burnside relations.

Let $W_{1}=W \cdot x_{1}$. Then $W_{1} \cdot p_{1} q p_{1}^{-1}=W_{1}$ and $W_{1} \cdot p\left(p_{1} q p_{1}^{-1}\right) p^{-1}=W_{1}$. By Lemma 6.10 applied to a $\left[\kappa(i)^{-1} \kappa(i)\right]$-sector of $W_{1}, p$ commutes with $p_{1} q p_{1}^{-1}$ modulo Burnside relations. Conjugating by $x_{1}$, we deduce that $g, h$ commute modulo Burnside relations.

Now we are ready to prove part (1) of Proposition 6.17 for $i=1$.

Recall that we can assume that all sectors of $W$ of the form $[\kappa(1) \rho(1)]$ are equal to $\kappa(1) \rho(1)$ in $H_{k a}^{*}$.

By Lemma 6.22, we can assume that there is no word $x$ such that $g=x p x^{-1}, h=x q x^{-1}$ modulo Burnside relations, where $p, q \in P$.

By Lemma 6.19(i), $h, g h g^{-1}$, and $b$ are $v$-trivial words. By Lemma 4.21 then $g$ is an $v$-trivial word. Hence by Lemma $6.19(\mathrm{i}), W_{1} \cdot g=W_{1}$ for every sector of $W$ of the form $[\kappa(1) \rho(1)]$. This implies that $W_{1} \cdot g^{s} h g^{-s}=W_{1}$ for every integer $s$. By Lemma 6.20, the same equality is true for every sector of $W$ of the form $\left[\kappa(1)^{-1} \kappa(1)\right]$. By Lemma $6.19(\mathrm{v})$ the same is true for sectors of the form $\left[\rho(1) \rho(1)^{-1}\right]$. Thus $W \cdot g^{s} h g^{-s}=W$ for every integer $s$.

This completes the proof of part (1) of Proposition 6.17.

Now let us prove part (2) of the proposition. So let us assume that there is no $i=1, \ldots, N$ such that each sector of $W$ is of one of the forms $[\kappa(i) \rho(i)],\left[\kappa(i)^{-1} \kappa(i)\right],\left[\rho(i) \rho(i)^{-1}\right]$. Assume that part (2) of Proposition 6.17 is not true and that $(|h|,|g|)$ is the smallest in lexicographic order pair of numbers for all such counterexamples $(h, g)$ (for any $W$ ). Clearly the word $h$ is not empty.

The next four lemmas allow us to improve out counterexample $(h, g)$.

Lemma 6.23. For every word $x$ over $\mathbb{S}$ such that $W$ is in the domain of $x$, the pair $\left(x^{-1} h x, x^{-1} g x\right)$ is also a counterexample to statement (2) of Proposition 6.17.

Proof. Indeed by Lemma $6.2(W \cdot x) \cdot x^{-1} h x=W \cdot x$,

$$
(W \cdot x) \cdot\left(x^{-1} g x\right)\left(x^{-1} h x\right)\left(x^{-1} g x\right)^{-1}=W \cdot x .
$$

Also since $W$ does not contain accepted subwords, $W \cdot x$ does not contain accepted subwords.

Lemma 6.24. The word $h$ is cyclically reduced.

Proof. Suppose that $h$ is not cyclically reduced. We can represent $h$ in the form $h_{1} h_{2}$ such that the reduced form $h^{\prime}$ of the word $h_{2} h_{1}$ is cyclically reduced and $\left|h^{\prime}\right|<|h|$. By Lemma 6.23, the pair $\left(h^{\prime}, h_{1}^{-1} g h_{1}\right)$ is also a counterexample to part (2) of Proposition 6.17. This contradicts the minimality of the counterexample $(h, g)$. Hence $h$ is cyclically reduced.

Lemma 6.25. There exists a possibly different counterexample $(\bar{h}, \bar{g})$ with $|\bar{h}|=|h|,|\bar{g}|=|g|$ such that $\bar{h}$ is cyclically reduced and $\bar{g} \bar{h} \bar{g}^{-1}$ is reduced. Moreover for some prefix $x^{-1}$ of the word $h$, we have $\bar{h}=x h x^{-1}, \bar{g}=x g x^{-1}$ in the free group. 
Proof. By Lemma 6.24, $h$ is cyclically reduced.

Therefore either $g h$ or $h g^{-1}$ is a freely reduced word. Let us assume that $h g^{-1}$ is freely reduced. The right-left dual of the argument presented below will work in the case when $g h$ is freely reduced.

Notice that $g \not \equiv g_{1} h^{-1}$ for any $g_{1}$, since otherwise $g_{1} h g_{1}^{-1}$ is freely equal to $g h g^{-1}$, and the counterexample $\left(h, g_{1}\right)$ would be smaller than $(g, h)$. Thus $g \equiv g_{1} f$ where $f^{-1} f^{\prime} \equiv h$ and $g_{1} f^{\prime}$ is a reduced word. Hence $g h g^{-1}=g_{1} f^{\prime} f^{-1} g_{1}^{-1}$, whence

$$
W \cdot g_{1} f^{\prime} f^{-1} g_{1}^{-1}=W
$$

Since $W \cdot f^{-1} f^{\prime}=W \cdot h=W$, we have $\left(W \cdot f^{-1}\right) \cdot f^{\prime} f^{-1}=W \cdot f^{-1}$. Let $W^{\prime}=W \cdot f^{-1}$. Then $W^{\prime} \cdot f^{\prime} f^{-1}=W^{\prime}$ and $W^{\prime} \cdot\left(f g_{1}\right) f^{\prime} f^{-1}\left(f g_{1}\right)^{-1}=W^{\prime}$ by $(21)$.

Now notice that since $h$ is cyclically reduced, $\left|f^{\prime} f^{-1}\right|=|h|$. If $\left|f g_{1}\right|<|g|$ then the minimality of $(|h|,|g|)$ implies that $W^{\prime} \cdot\left(f g_{1}\right)=W^{\prime}$ or $f g_{1}$ commutes with $f^{\prime} f^{-1}$ modulo Burnside relations. In the first case $W \cdot g_{1} f=W$. Since $W \cdot h=W$, we get $W \cdot g=W \cdot\left(g_{1} f\right)=W$, a contradiction. In the second case conjugating by $f^{-1}$, we get that $g_{1} f$ commutes with $f^{-1} f^{\prime}=h$ modulo Burnside relations. Therefore $g \equiv g_{1} f$ commutes with $h$ modulo Burnside relations, a contradiction.

Hence the length of the freely reduced form of $f g_{1}$ is equal to $|g|$. This implies that if $g_{1}$ is not empty then $g$ is cyclically reduced (since $f$ is not empty by our assumption).

Case 1. Suppose that $g_{1}$ is not empty. Then the word $f g_{1} f^{\prime} f^{-1} g_{1}^{-1} f^{-1}$ is reduced $\left(f g_{1}\right.$ is reduced because $g \equiv g_{1} f,\left|f g_{1}\right|=|g|, g_{1} f^{\prime}$ is reduced by the definition, $f^{\prime} f^{-1}$ is reduced because it is a cyclic shift of the cyclically reduced word $h, f^{-1} g_{1}^{-1}$ is reduced because $g_{1} f \equiv g$ is reduced). In this case $\bar{h} \equiv f^{\prime} f^{-1}, \bar{g} \equiv f g_{1}$ and $x \equiv f$. Therefore $W$ is in the domain of $x^{-1}$. By Lemma 6.23, the pair $(\bar{h}, \bar{g})$ is a (minimal) counterexample, as required.

Case 2. Now let $g_{1}$ be empty, that is $f \equiv g$. If $g$ is not cyclically reduced, then $g \equiv y g_{2} y^{-1}$, $h \equiv y g_{2}^{-1} y^{-1} h_{2}$ for some $g_{2}, h_{2}$ and a non-empty $y$. Applying Lemma 6.23 for $x \equiv y$, we obtain a counterexample $\left(g_{2} y^{-1} h_{2} y, g_{2}\right)$. Since $g_{2}$ is shorter than $g$, and $g_{2} y^{-1} h_{2} y$ is of the same length as $h$, we get a contradiction. Hence $g$ is cyclically reduced.

Now let $g^{-s}$ be the maximal power of $g$ which is a prefix of $h$. Using Lemma 6.23 with $x \equiv g^{-s}$, we can pass from the pair $(h, g)$ to another minimal counterexample $(\hat{h}, g)=\left(h_{1} g^{-s}, g\right)$ where $h_{1}$ is defined by the formula $h \equiv g^{-s} h_{1}$. Notice that since $h$ is cyclically reduced by Lemma $6.24, h_{1} g^{-s}$ is cyclically reduced. The word $h_{1}$ is not trivial because otherwise $g$ and $h$ would commute, so $(h, g)$ would not be a counterexample. Since $g$ is cyclically reduced, the product $\hat{h} g^{-1}$ is reduced. If $g \hat{h}$ is reduced, the product $g \hat{h} g^{-1}$ is reduced and we are done. Otherwise, since $g^{-1}$ is not a prefix of $\hat{h}$ (by the choice of $s$ ), we can apply the argument of Case 1 and produce the required counterexample.

Lemma 6.25 allows us to assume (from now on) that $\mathrm{ghg}^{-1}$ is reduced.

Lemma 6.26. The words $g, h$ are Burnside reduced.

Proof. Let $h$ contain a non-empty subword $c$ which is equal to 1 modulo Burnside relations. Then $h \equiv h_{1} c h_{2}$ for some $h_{1}, h_{2}$. By Lemma 6.5 (iii), $W \cdot h_{1} h_{2}=W$. Since $g h g^{-1}$ is reduced, we have $W \cdot g \cdot h_{1} c h_{2} \cdot g^{-1}=W$, so $W \cdot g h_{1} h_{2} g^{-1}=W$. Now it is clear that $\left(h_{1} h_{2}, g\right)$ is a counterexample to part (2) of Proposition 6.17 , which contradicts the minimality of $(h, g)$. Similarly if $g$ is not Burnside-reduced, we can shorten $g$. 
Lemma 6.27. One cannot represent $h$ as a product $h_{1} h_{2} h_{3}$ where $h_{1}=h_{3}^{-1}$ modulo Burnside relations and $h_{1}, h_{3}$ consist of rules from the same $\mathbb{S}_{\omega}, \omega \in \Omega$, and at least one of the words $h_{1}$ or $h_{3}$ is not empty.

Proof. If one of the words $h_{1}$ or $h_{3}$ is empty then $h_{1}=h_{3}=1$ modulo Burnside relations, and we get a contradiction with Lemma 6.26. Hence both $h_{1}$ and $h_{3}$ are not empty. Let $f$ be a shortest word among $h_{1}, h_{3}^{-1}$. By Lemma $6.6, h_{1}, h_{3}^{-1}, f$ define the same transformation from $P(\mathbb{S})$. Hence we have

$$
W \cdot h=W \cdot h_{1} \cdot h_{2} \cdot h_{3}=W \cdot f \cdot h_{2} \cdot f^{-1}=W \cdot f h_{2} f^{-1}=W
$$

and, since $g h g^{-1}$ is reduced,

$$
W \cdot g \cdot h_{1} \cdot h_{2} \cdot h_{3} \cdot g^{-1}=W \cdot g\left(f h_{2} f^{-1}\right) g^{-1}=W .
$$

Since $h=f h_{2} f^{-1}$ modulo Burnside relations, the pair $\left(f h_{2} f^{-1}, g\right)$ is a counterexample to part (2) of Proposition 6.17. Since $|f| \leq\left|h_{1}\right|,\left|h_{3}\right|,\left(f h_{2} f^{-1}, g\right)$ is a minimal counterexample. But $f h_{2} f^{-1}$ is not cyclically reduced, a contradiction with Lemma 6.24.

Lemma 6.28. Suppose that $\mathrm{ghg}^{-1}$ contains a connecting rule $\mathbf{c}_{\omega}^{ \pm 1}$ which locks a sector $z w z^{\prime}$ of $W$, $z \not \equiv \kappa(1), \rho(1)^{-1}$. Suppose that all rules in ghg $^{-1}$ which differ from $\mathbf{c}_{\omega}^{ \pm 1}$ are left or right active for $z$-sectors. Then ghg $^{-1}$ contains at least two different connecting rules $\mathbf{c}_{i}^{ \pm 1}$ and $\mathbf{c}_{i+1}^{ \pm 1}, i \in \Omega$.

Proof. By Lemma 6.9, $z^{\prime} \equiv z_{+} ; z \not \equiv \kappa(i), \rho(i)^{-1}$ for any $i$ because all rules lock $\kappa(i)$-sectors if $i>1$, and $z \not \equiv \kappa(1), \rho(1)^{-1}$ by assumption. Hence every connecting rule changes the $\Omega$-coordinate of $z$-sectors.

Suppose, by contradiction, that $g h g^{-1}$ does not contain two different connecting rules. Since $W \cdot g h g^{-1}=W$ and $W \cdot h=W$, and the word $g h g^{-1}$ is reduced, $W$ and $W \cdot g$ have the same $\Omega$ coordinate. Hence either $g$ does not contain $\mathbf{c}_{\omega}^{ \pm 1}$ or $g$ contains a subword $\mathbf{c}_{\omega}^{ \pm 1} f \mathbf{c}_{\omega}^{\mp 1}$ where $f$ contains no connecting rules. In the first case, $h$ contains $\mathbf{c}_{\omega}^{ \pm 1}$, so (since $W \cdot h=W$ ), $h$ contains a subword of the form $\mathbf{c}_{\omega}^{ \pm 1} f \mathbf{c}_{\omega}^{\mp 1}$. In both cases $f$ contains rules from only one $\mathbb{S}_{i}$. All these rules are active for $z$-sectors by our assumption. Hence by Lemma 6.11 (which can be applied since $z \not \equiv \kappa(1), \rho(1)^{-1}$ ), $f=1$ modulo Burnside relations, a contradiction with Lemma 6.26.

Lemma 6.29. $W$ cannot contain a sector of the form $\left[\vec{\kappa}(i, j) \vec{\kappa}(i, j)^{-1}\right]$.

Proof. Suppose that $W$ contains such a sector $W^{\prime} \equiv \vec{\kappa}(i, j, \omega) w \vec{\kappa}(i, j, \omega)^{-1}$. Notice that since $W$ starts and ends with the same $\mathbf{K}$-letter, it must contain a sector $W^{\prime \prime}$ of the form $z^{-1} w^{\prime \prime} z, z \in \overline{\mathbf{K}}(\omega)$, either to the left or to the right of our sector $W^{\prime}$. Replacing $W$ by $W^{-1}$ if necessary, we can assume that such a sector occurs to the left of $W^{\prime}$. We also choose $W^{\prime \prime}$ to be as close to $W^{\prime}$ on the left as possible.

By Lemma $6.9, \mathrm{ghg}^{-1}$ does not contain occurrences of $\mathbf{c}_{\omega}$ or rules from $\mathbb{S}_{\omega}$ which lock $\vec{\kappa}(i, j)$ sectors. For every $t \in \Omega$ either $\mathbf{c}_{t}$ or $\mathbf{c}_{t+1}$ locks $\vec{\kappa}(i, j)$-sectors, and $\mathbf{c}_{2 n}$ and every rule from $\mathbb{S}_{2 n+1}$ lock these sectors. Therefore $g h g^{-1}$ can only contain rules from two consecutive $\mathbb{S}_{t}, \mathbb{S}_{t+1}(\omega=t$ or $\omega=t+1$ ), and does not contain rules from $\mathbb{S}_{2 n+1} \cup\left\{\mathbf{c}_{2 n}, \mathbf{c}_{2 n}^{-1}\right\}$.

If $g h g^{-1}$ contains rules only from one $\mathbb{S}_{t}$, then by Lemma 6.10 (ii), we have that $h$ and $g h g^{-1}$ are equal modulo Burnside relations to powers of $\operatorname{root}_{\omega}(w)$. By Lemma 4.19, it follows that $h$ and $g$ commute modulo Burnside relations, a contradiction. 
Thus we can assume that $g h g^{-1}$ contains rules from two different sets $\mathbb{S}_{t}$ and $\mathbb{S}_{t+1}$ or $g h g^{-1} \equiv$ $\mathbf{c}_{t}^{ \pm 1} f \mathbf{c}_{t}^{\mp 1}$ for some $f$. In both cases $g h g^{-1}$ contains an occurrence of $\mathbf{c}_{t}$ and an occurrence of $\mathbf{c}_{t}^{-1}$.

Since $W^{\prime}$ is not a prefix of $W$, by the definition of admissible words, $W$ contains a subword of one of the following forms:

$$
\begin{aligned}
& \vec{\kappa}(i, j, \omega)^{-1} w_{1} \vec{\kappa}(i, j, \omega) w \vec{\kappa}(i, j, \omega)^{-1}, \\
& \overleftarrow{\kappa}(i, j-1, \omega) w_{1} \vec{\kappa}(i, j, \omega) w \vec{\kappa}(i, j, \omega)^{-1} \quad(j>1), \\
& \rho(i) w_{1} \vec{\kappa}(i, 1, \omega) w \vec{\kappa}(i, 1, \omega)^{-1}
\end{aligned}
$$

for some word $w_{1}$ over $A\left(\vec{\kappa}(i, j)_{-}\right)$.

Consider each of these cases.

Case 1. $W$ contains $\vec{\kappa}(i, j, \omega)^{-1} w_{1} \vec{\kappa}(i, j, \omega) w \vec{\kappa}(i, j, \omega)^{-1}$ for some $w$.

Case 1.1. Let $j>1$. Then $\vec{\kappa}(i, j)_{-}=\overleftarrow{\kappa}(i, j-1)$. Since for every $t \geq 0$ if $\mathbf{c}_{t}$ is active for $\vec{\kappa}(i, j)$-sectors then it locks $\overleftarrow{\kappa}(i, j-1)$-sectors, $\mathbf{c}_{t}$ locks $\overleftarrow{\kappa}(i, j-1)$-sectors. This contradicts Lemma 6.9 .

Case 1.2. Let $j=1$. Then $\vec{\kappa}(i, j)_{-} \equiv \rho(i)$. Then by Lemma $6.9, t, t+1 \neq 0$. Recall also that $t, t+1 \neq 2 n+1$ and that all other (working) rules except for rules from $\mathbb{S}_{0} \cup\left\{\mathbf{c}_{0}\right\}$ are right active for $\rho(i)$-sectors. By Lemma 6.10 (ii) $h$ and $g h g^{-1}$ are a powers of $\operatorname{root}_{\omega}\left(w_{1}\right)$ modulo Burnside relations, so by Lemma 4.19, $h$ and $g$ commute modulo Burnside relations, a contradiction.

Case 2. $W$ contains $\overleftarrow{\kappa}(i, j-1, \omega) w_{1} \vec{\kappa}(i, j, \omega) w \vec{\kappa}(i, j, \omega)^{-1}, j>1$. Then $\mathbf{c}_{t}$ locks $\overleftarrow{\kappa}(i, j-1)-$ sectors (indeed, every connecting rule which does not lock some $\vec{\kappa}(s, l)$-sectors locks all $\overleftarrow{\kappa}(s, l)$ sectors). Lemma 6.28 now implies that rules of one of the sets $\mathbb{S}_{t}$ or $\mathbb{S}_{t+1}$ lock $\overleftarrow{\kappa}(i, j-1)$-sectors. Let $\mathbb{S}_{t^{\prime}}, t^{\prime} \in\{t, t+1\}$ consist of rules which lock $\overleftarrow{\kappa}(i, j-1)$-sectors. By definition of $\mathbb{S}, t^{\prime} \in$ $\{0,2 j-1,2 j, 2 j+1, \ldots, 2 n+1\}$. Since for $t^{\prime} \in\{2 j, \ldots, 2 n+1\}$ the rules of $\mathbb{S}_{t^{\prime}}$ lock also $\vec{\kappa}(i, j)$ sectors, we conclude that $t^{\prime} \in\{0,2 j-1\}$.

Case 2.1. Let $t^{\prime}=2 j-1, j>1$. Then $t^{\prime}=t+1$ because rules from $\mathbb{S}_{2 j}$ lock $\vec{\kappa}(i, j)$-sectors. So $t=2 j-2$. Since all rules from $\mathbb{S}_{2 j-1}$ lock all $\vec{\kappa}(i, 1)-, \overleftarrow{\kappa}(i, 1)-, \ldots, \overleftarrow{\kappa}(i, j-1)$-sectors, none of the letters $\vec{\kappa}(i, 1, \omega), \overleftarrow{\kappa}(i, 1, \omega), \ldots, \overleftarrow{\kappa}(i, j-1, \omega)$ belong to the sector $W^{\prime \prime}$ (by Lemma 6.9 ). The definition of admissible words now implies that in $W$, K-letters to the left of $\overleftarrow{\kappa}(i, j-1, \omega)$ are placed in the natural (decreasing) order from $\overleftarrow{\kappa}(i, j-1, \omega)$ to $\vec{\kappa}(i, 1, \omega)$. The $\mathbf{K}$-letter in $W$ next to the left of $\vec{\kappa}(i, 1, \omega)$ may be either $\rho(i)$ or $\vec{\kappa}(i, 1, \omega)^{-1}$. Consider both cases.

Case 2.1.1. Suppose that $W$ contains a sector of the form $\rho(i) w^{\prime \prime \prime} \vec{\kappa}(i, 1, \omega)$. Since neither $t$ nor $t+1$ are equal to 0 or $2 n+1$ (recall that $t^{\prime} \in\{t, t+1\}, t^{\prime}=2 j-1, j>1$ ), all rules from $\mathbb{S}_{t} \cup \mathbb{S}_{t+1} \cup\left\{\mathbf{c}_{t}\right\}$ are right active for the $\rho(i)$-sectors and (by Lemma 6.4) $L^{a}(h, \vec{\kappa}(i, 1)$ ) is a copy of $h$. Since $W \cdot h=W$, we conclude that $h=1$ modulo Burnside relations, a contradiction.

Case 2.1.2. Suppose that $W$ contains a sector of the form $\vec{\kappa}(i, 1, \omega)^{-1} w^{\prime \prime \prime} \vec{\kappa}(i, 1, \omega)$. Then by Lemma $6.10 h$ and $g h g^{-1}$ belong to the same cyclic subgroup generated by $\operatorname{root}_{\omega}\left(w^{\prime \prime \prime}\right)$ modulo Burnside relations. As before, by Lemma 4.19, this implies that $h$ and $g$ commute modulo Burnside relations, a contradiction.

Case 2.2. Let $t^{\prime}=0$. Then $g h g^{-1}$ contain rules from $\mathbb{S}_{0} \cup \mathbb{S}_{1} \cup\left\{\mathbf{c}_{0}, \mathbf{c}_{0}^{-1}\right\}$ only.

Notice that $g$ cannot contain a subword of the form $\mathbf{c}_{0} g_{1} \mathbf{c}_{0}^{-1}$ where $g_{1}$ is a word over $\mathbb{S}_{1}$. Indeed otherwise by Lemma 6.11 applied to the sector $\overleftarrow{\kappa}(i, j-1, \omega) w_{1} \vec{\kappa}(i, j, \omega)$ of $W$, we get $g_{1}=1$ modulo Burnside relations (since $\mathbf{c}_{0}$ locks this sector, and all rules from $\mathbb{S}_{1}$ are left active for it). 
This would contradict Lemma 6.26. Similarly $h$ cannot contain a subword of the form $\mathbf{c}_{0} h_{1} \mathbf{c}_{0}^{-1}$ where $h_{1}$ consists of rules from $\mathbb{S}_{1}$. Hence either $h$ contains rules from only one $\mathbb{S}_{\omega}$ or $h$ has the form $h_{1} \mathbf{c}_{0}^{-1} h_{2} \mathbf{c}_{0} h_{3}$ where $h_{1}, h_{3}$ are possibly empty words over $\mathbb{S}_{1}, h_{2}$ is a word over $\mathbb{S}_{0}$.

Since $W$ and $W \cdot g$ are in the domain of $h, W$ and $W \cdot g$ have the same $\Omega$-coordinate. Hence the total degree of $\mathbf{c}_{0}$ in $g$ is 0 . Therefore either $g$ does not contain $\mathbf{c}_{0}$ and $\mathbf{c}_{0}^{-1}$ or $g$ has the form $g_{1} \mathbf{c}_{0}^{-1} g_{2} \mathbf{c}_{0} g_{3}$ where $g_{1}, g_{3}$ are words over $\mathbb{S}_{1}$ (these words can be empty) and $g_{2}$ is a non-empty word over $\mathbb{S}_{0}$.

Consider these two cases separately.

Case 2.2.1. Suppose that $g$ does not contain $\mathbf{c}_{0}$ and $\mathbf{c}_{0}^{-1}$. Since $g h g^{-1}$ must contain $\mathbf{c}_{0}$ or $\mathbf{c}_{0}^{-1}$, $h$ has the form $h_{1} \mathbf{c}_{0}^{-1} h_{2} \mathbf{c}_{0} h_{3}$ where $h_{1}, h_{3}$ are words over $\mathbb{S}_{1}, h_{2}$ is a word over $\mathbb{S}_{0}$. Since $W \cdot h=W$, the $\Omega$-coordinate of $W$ is 1 , and $g$ is a word over $\mathbb{S}_{1}$.

Let us apply Lemma 6.4 (i) to the sector $\overleftarrow{\kappa}(i, j-1, \omega) w_{1} \vec{\kappa}(i, j, \omega)$ of $W$. Since $\mathbf{c}_{0}$ locks this sector, $R^{a}\left(g h_{1}, \overleftarrow{\kappa}(i, j-1)\right)=R^{a}\left(h_{1}, \overleftarrow{\kappa}(i, j-1)\right)=w_{1}$ modulo Burnside relations. By Lemma 6.4 (iii), we obtain that $g h_{1}=h_{1}$ modulo Burnside relations, so $g=1$ modulo Burnside relations, a contradiction.

Case 2.2.2. Suppose that $g \equiv g_{1} \mathbf{c}_{0}^{-1} g_{2} \mathbf{c}_{0} g_{3}$ where $g_{1}, g_{3}$ are words over $\mathbb{S}_{1}, g_{2}$ is a word over $\mathbb{S}_{0}$.

If $h$ consists of rules from $\mathbb{S}_{1}$, then we can apply Lemma 6.11 to the subword $g_{3} h g_{3}^{-1}$ of $g h g^{-1}$ and a sector of the type $[\overleftarrow{\kappa}(i, j-1) \vec{\kappa}(i, j)]$ of $W \cdot g_{1} \mathbf{c}_{0}^{-1} g_{2} \mathbf{c}_{0}$ to obtain that $g_{3} h g_{3}^{-1}$ is equal to 1 modulo Burnside relations. Hence $h=1$ modulo Burnside relations, a contradiction.

Since $h$ does not have subwords of the form $c_{0} \bar{h} c_{0}^{-1}, h$ has the form $h_{1} \mathbf{c}_{0}^{-1} h_{2} \mathbf{c}_{0} h_{3}$ where $h_{1}, h_{3}$ are words over $\mathbb{S}_{1}, h_{2}$ is a word over $\mathbb{S}_{0}$. Then again by Lemma $6.11, g_{3} h_{1}=1, h_{3} g_{3}^{-1}=1$ modulo Burnside relations. Hence $h_{1}=h_{3}^{-1}$ modulo Burnside relations, which contradicts Lemma 6.27 if $h_{1}$ or $h_{3}$ are not empty or Lemma 6.24 if $h_{1}$ and $h_{3}$ are empty.

Remark 6.30. Notice that the only properties of the sector $\overleftarrow{\kappa}(i, j-1, \omega) w_{1} \vec{\kappa}(i, j, \omega)$ used in Case 2.2 are the properties that all rules from $\mathbb{S}_{0} \cup\left\{\mathbf{c}_{0}\right\}$ lock this sector and all rules from $\mathbb{S}_{1}$ are active for this sector.

Case 3. Let $W$ contain sectors $\rho(i) w_{1} \vec{\kappa}(i, 1, \omega)$ and $\vec{\kappa}(i, 1, \omega) w \vec{\kappa}(i, 1, \omega)^{-1}$. Recall that $t, t+1 \neq 2 n+1$.

Case 3.1. Suppose that $t \neq 0$. Then all rules from $\mathbb{S}_{t} \cup \mathbb{S}_{t+1} \cup\left\{\mathbf{c}_{t}\right\}$ are right active for $\rho(i)$-sectors. Therefore by Lemma $6.12 h=1$ modulo Burnside relations, a contradiction.

Case 3.2. Let $t=0$. By Remark 6.30, this case is similar to Case 2.2 because all rules from $\mathbb{S}_{0} \cup\left\{\mathbf{c}_{0}\right\}$ lock $\rho(i)$-sectors and all rules from $\mathbb{S}_{1}$ are (right) active for these sectors.

Lemma 6.31. W cannot contain a sector of the form $\left[\overleftarrow{\kappa}(i, j) \overleftarrow{\kappa}(i, j)^{-1}\right], i=1, \ldots, N, j=1, \ldots, n$.

Proof. Suppose that $W$ contains a sector $W^{\prime}$ of the form $\overleftarrow{\kappa}(i, j, \omega) w \overleftarrow{\kappa}(i, j, \omega)^{-1}, i=1, \ldots, N, j=$ $1, \ldots, n, \omega \in \Omega$. As in the proof of Lemma 6.29 , we can assume that $W$ contains a sector $W^{\prime \prime}$ of the form $\left[z^{-1} z\right]$ to the left of $W^{\prime}, z \in \mathbf{K}$. We also assume that $W^{\prime \prime}$ is the closest to $W^{\prime}$ such sector (in principle, $W^{\prime \prime}$ can have a common letter $\overleftarrow{\kappa}(i, j, \omega)$ with $\left.W^{\prime}\right)$.

Since all rules from $\mathbb{S}_{0} \cup \mathbb{S}_{2 n+1} \cup\left\{\mathbf{c}_{2 i}, i=0, \ldots, n\right\}$ lock $\overleftarrow{\kappa}(i, j)$-sectors, none of these rules can occur in $g h g^{-1}$ by Lemma 6.9. Therefore $g h g^{-1}$ involves rules from at most two sets $\mathbb{S}_{t}, \mathbb{S}_{t+1}$. 
If $g h g^{-1}$ contains rules of only one set $\mathbb{S}_{\omega}$ then by Lemma $6.13(h, g)$ is not a counterexample to part (2) of Proposition 6.17, a contradiction.

Thus $g h g^{-1}$ contains $\mathbf{c}_{t}$ for some $t$. Moreover this $t$ must be odd because connecting rules with even indices lock $\overleftarrow{\kappa}(i, j)$-sectors.

Then $\mathbf{c}_{t}$ locks $\vec{\kappa}(k, \ell)$-sectors, $1 \leq k \leq N, 1 \leq \ell \leq n$. So by Lemma $6.9, z \not \equiv \overleftarrow{\kappa}(k, \ell)$ for any $k, \ell$.

Hence the first letter $\overleftarrow{\kappa}(i, j, \omega)$ of $W^{\prime}$ does not belong to $W^{\prime \prime}$. Therefore $W$ contains a sector of the form $[\vec{\kappa}(i, j) \overleftarrow{\kappa}(i, j)]$. If rules from $\mathbb{S}_{t}$ and $\mathbb{S}_{t+1}$ are active for $\vec{\kappa}(i, j)$-sectors, and $\mathbf{c}_{t}$ locks these sectors, we get a contradiction with Lemma 6.28.

Hence rules from $\mathbb{S}_{t+1}$ must lock $\vec{\kappa}(i, j)$-sectors. Therefore these rules lock all $\vec{\kappa}\left(i, j^{\prime}\right)$ - and $\overleftarrow{\kappa}\left(i, j^{\prime}\right)$-sectors with $j^{\prime}<j$. Hence $z \not \equiv \vec{\kappa}\left(i, j^{\prime}\right), j^{\prime}=2,3, \ldots, j$ and $z \not \equiv \overleftarrow{\kappa}\left(i, j^{\prime}\right), j^{\prime}=1,2, \ldots, j$. Hence the K-letter preceding $\overleftarrow{\kappa}(i, j, \omega)$ in $W$ is $\vec{\kappa}(i, j, \omega)$, the $\mathbf{K}$-letter preceding $\vec{\kappa}(i, j, \omega)$ is $\overleftarrow{\kappa}(i, j-1, \omega)$, etc. We conclude that $W$ contains a sector of one of the forms $[\rho(i) \vec{\kappa}(i, 1)]$ or $\left[\vec{\kappa}(i, 1)^{-1} \vec{\kappa}(i, 1)\right]$.

In the first case we get a contradiction with Lemma 6.7. In the second case by Lemma 6.10 $h, g h g^{-1}$ belong to the same cyclic subgroup modulo Burnside relations, and we get a contradiction using Lemma 4.19 .

Lemma 6.32. Not all sectors of $W$ have the form $\left[z z^{-1}\right]$ where $z$ is one of the letters $\lambda(i, \omega)$, $\lambda(i, \omega)^{-1}, \rho(i), \rho(i)^{-1}$.

Proof. Suppose that each sector of $W$ has one of these forms. But for each

$$
z \in\left\{\lambda(i, \omega), \lambda(i, \omega)^{-1}, \rho(i), \rho(i)^{-1}\right\}
$$

$L^{a}(\tau, z)=R^{a}(\tau, z)=1$ for every $\tau \in \mathbb{S}$. Notice that $W$ is in the domain of $g$ because $g h g^{-1}$ is reduced and $W \cdot g h g^{-1}=W$. Now by Lemma 6.4 we have $W \cdot g=W$, a contradiction.

Lemma 6.33. $W$ does not contain sectors of the form $\left[\kappa(i) \kappa(i)^{-1}\right]$.

Proof. If $i>1$ then the statement immediately follows from Lemma 6.9 because all rules in $\mathbb{S}$ lock $\kappa(i)$-sectors. So let $i=1$ and suppose that $W$ contains a sector of the form $\left[\kappa(1) \kappa(1)^{-1}\right]$. Then by Lemma $6.9 \mathrm{ghg}^{-1}$ does not contain rules from $\mathbb{S}_{0}$ and $\mathbb{S}_{2 n+1}$ and connecting rules $\mathbf{c}_{0}^{ \pm 1}, \mathbf{c}_{2 n}^{ \pm 1}$. As before we may assume that there is a sector $W^{\prime \prime}$ of the form $z^{-1} w_{1} z$ to the left of $W^{\prime}$ in $W$ (if not we replace $W$ by $\left.W^{-1}\right)$. In particular, $W^{\prime}$ is not a prefix of $W$. The $\mathbf{K}$-letter preceding $\kappa(1)$ of $W^{\prime}$ in $W$ is either $\kappa(1)^{-1}$ or $\lambda(1, \omega)$. In the first case we get a contradiction using Lemma 6.10 (ii) and Lemma 6.19(iii) because by Lemma 6.4 (iii) $\vartheta_{1}\left(g h g^{-1}\right)$ is a copy of $g h g^{-1}$ in this case. In the second case we get a contradiction with Lemma 6.7 .

Lemma 6.34. $W$ does not contain sectors of the form $z^{-1} w z$, where $z \equiv \vec{\kappa}(i, j, \omega)$ for $j \neq 1$ or $z \equiv \overleftarrow{\kappa}(i, j, \omega)$ or $z \equiv \lambda(i, \omega)$

Proof. Indeed, suppose that $W$ contains such a sector $W^{\prime}$. Then by Lemma 6.9 , rules from $\mathbb{S}_{2 n+1} \cup$ $\left\{\mathbf{c}_{2 n}, \mathbf{c}_{2 n}^{-1}\right\}$ do not occur in $g h g^{-1}$ if $z \equiv \vec{\kappa}(i, j, \omega)$ for $j \neq 1$ or $z \equiv \overleftarrow{\kappa}(i, j, \omega)$ or $z \equiv \lambda(i, \omega)$.

Since $W$ starts and ends with the same $\mathbf{K}$-letter, we can assume that $W$ contains a sector $W^{\prime \prime}$ of the form $z_{1} w_{1} z_{1}^{-1}$ to the right of $W^{\prime}$. By Lemmas 6.29 and 6.31 the $\mathbf{K}$-letter $z_{1}$ in $W^{\prime \prime}$ can be of one of the forms $\lambda(s, \omega), \kappa(s), \rho(s)$ for some $s$. By Lemma 6.9 this implies that rules from $\mathbb{S}_{0}$ and 
$\mathbf{c}_{0}$ cannot occur in $g h g^{-1}$. As in the proofs of Lemmas 6.29,6.31, all rules in $g h g^{-1}$ are contained in $\mathbb{S}_{t} \cup \mathbb{S}_{t+1} \cup\left\{\mathbf{c}_{t}, \mathbf{c}_{t}^{-1}\right\}, t \neq 0,2 n$.

Case 1. Suppose that the $\mathbf{K}$-letter in $W^{\prime}$ is $\vec{\kappa}(i, j, \omega)$. Then by Lemma 6.29 the $\mathbf{K}$-letter next to the right of $W^{\prime}$ cannot be $\vec{\kappa}(i, j, \omega)^{-1}$, so it must be $\overleftarrow{\kappa}(i, j, \omega)$. But rules from $\mathbb{S}_{t} \cup \mathbb{S}_{t+1}$ are active for $\vec{\kappa}(i, j)$-sectors and the rule $\mathbf{c}_{t}$ locks this sector. Thus we get a contradiction with Lemma 6.28 .

Case 2. Suppose now that $z \equiv \overleftarrow{\kappa}(i, j, \omega)$. Then by Lemma 6.31 , the K-letter next to the right of $W^{\prime}$ cannot be $\overleftarrow{\kappa}(i, j, \omega)^{-1}$, so it must be $\vec{\kappa}(i, j+1, \omega)$ if $j<n$ or $\lambda(i+1, \omega)$ if $j=n$. As in Case 1 , rules from $\mathbb{S}_{t} \cup \mathbb{S}_{t+1}$ are active for $\overleftarrow{\kappa}(i, j)$-sectors, and the rule $\mathbf{c}_{t}$ locks these sectors, which contradicts Lemma 6.28.

Case 3. Suppose that the K-letter in $W^{\prime}$ is $\lambda(i, \omega)$. Since by Lemma 6.32 not all sectors of $W$ have the form $z w z^{-1}$ where $z \in\left\{\lambda(i, \omega), \lambda(i, \omega)^{-1}\right\}, W$ either has a sector of the form $[\lambda(i) \kappa(i)]$ or it has sectors of the forms $\left[\lambda(i) \lambda(i)^{-1}\right]$ and $[\overleftarrow{\kappa}(i-1, n) \lambda(i)]$.

Case 3.1. Suppose that $W$ has a sector of the form $[\lambda(i) \kappa(i)]$. Recall that rules from $\mathbb{S}_{0}, \mathbf{c}_{0}, \mathbf{c}_{2 n}$ do not occur in $g h g^{-1}$. All other rules are right active for $\lambda(i)$-sectors. This by Lemma 6.7 implies that $g h g^{-1}=1$ modulo Burnside relations, a contradiction.

Case 3.2. Suppose that $W$ has a sector of the form $\left[\lambda(i) \lambda(i)^{-1}\right]$ and a sector of the form $[\overleftarrow{\kappa}(i-1, n) \lambda(i)]$. Since we have already proved (Cases 1,2$)$ that $W$ does not contain sectors

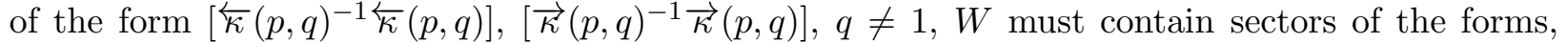
$[\vec{\kappa}(i-1, n) \overleftarrow{\kappa}(i-1, n)],[\overleftarrow{\kappa}(i-1, n-1) \vec{\kappa}(i-1, n)], \ldots,[\vec{\kappa}(i-1,1) \overleftarrow{\kappa}(i-1,1)]$. Thus $W$ contains $\vec{\kappa}(i-1,1, \omega)^{-1}$. The $\mathbf{K}$-letter next to the right of $\vec{\kappa}(i-1,1, \omega)^{-1}$ in $W$ is either $\vec{\kappa}(i-1,1, \omega)$ or $\rho(i-1)^{-1}$. Since all rules from $\mathbb{S}_{t} \cup \mathbb{S}_{t+1} \cup\left\{\mathbf{c}_{t}\right\}$ are left active for $\vec{\kappa}(i-1,1)^{-1}$-sectors, in the first case we get a contradiction by using Lemma 6.10(ii) and Lemma 4.19. In the second case we get a contradiction with Lemma 6.7.

Lemma 6.35. If $W$ contains a sector $W^{\prime}$ of the form $[\vec{\kappa}(i, n) \overleftarrow{\kappa}(i, n)]$ and a sector $W^{\prime \prime}$ of the form $[\overleftarrow{\kappa}(i, n) \lambda(i+1)]$, and $h$ contains a subword $h^{\prime}$ of the form $\mathbf{c}_{s}^{ \pm 1} h_{1} \mathbf{c}_{s}^{\mp 1}$, where $h_{1}$ does not contain either connecting rules or rules from $\mathbb{S}_{2 n+1}$, then $h_{1}$ contains rules from $\mathbb{S}_{0}$.

Proof. Indeed suppose that $h_{1}$ does not contain rules from $\mathbb{S}_{0}$. Notice that $\mathbf{c}_{s}$ locks either $W^{\prime}$ or $W^{\prime \prime}$, but all rules from $\mathbb{S} \backslash\left(\mathbb{S}_{0} \cup \mathbb{S}_{2 n+1} \cup\left\{\mathbf{c}_{0}, \ldots, \mathbf{c}_{2 n}\right\}\right)$ are left active for $\vec{\kappa}(i, n)$-sectors and for $\overleftarrow{\kappa}(i, n)$-sectors. Hence we can apply Lemma 6.11 to either $W^{\prime}$ or $W^{\prime \prime}, h_{1}$ is equal to 1 modulo Burnside relations which contradicts Lemma 6.26.

Lemma 6.36. If $W$ contains a sector of the form $[\vec{\kappa}(i, n) \overleftarrow{\kappa}(i, n)]$ and a sector of the form $[\overleftarrow{\kappa}(i, n) \lambda(i+1)]$ then $h$ contains a rule from $\mathbb{S}_{0} \cup\left\{\mathbf{c}_{0}\right\}$ or $h$ is a word over $\mathbb{S}_{2 n+1}$.

Proof. Let $\omega$ be the $\Omega$-coordinate of $W$. Suppose $h$ does not contain rules from $\mathbb{S}_{0}$ and does not consist of rules from $\mathbb{S}_{2 n+1}$.

Then by Lemma $6.35, h$ has the form

$$
h_{\omega} \mathbf{c}_{\omega} h_{\omega+1} \ldots h_{2 n} \mathbf{c}_{2 n} h_{2 n+1} \mathbf{c}_{2 n}^{-1} h_{2 n}^{\prime} \ldots \mathbf{c}_{\omega}^{-1} h_{\omega}^{\prime}
$$

where $h_{j}$ and $h_{j}^{\prime}$ are words over $\mathbb{S}_{j}, j=\omega, \ldots, 2 n+1$. 
The connecting rule $\mathbf{c}_{\omega}$ locks either $\vec{\kappa}(i, n)$-sectors or $\overleftarrow{\kappa}(i, n)$-sectors. The rule $\mathbf{c}_{2 n}$ locks both $\vec{\kappa}(i, n)$-sectors and $\overleftarrow{\kappa}(i, n)$-sectors. All rules from $\mathbb{S}_{\omega}, \omega<2 n$, are left active for $\overleftarrow{\kappa}(i, n)$-sectors, and for $\vec{\kappa}(i, n)$-sectors, rules from $\mathbb{S}_{2 n}$ are left active for $\overleftarrow{\kappa}(i, n)$-sectors. Hence by Lemma 6.4(iii) $h_{\omega}=\left(h_{\omega}^{\prime}\right)^{-1}$ modulo Burnside relations. By Lemma $6.27, h_{\omega}$ and $h_{\omega}^{\prime}$ are empty. Then $h$ is not cyclically reduced (it starts with $\mathbf{c}_{\omega}$ and ends with $\mathbf{c}_{\omega}^{-1}$, a contradiction).

Lemma 6.37. Suppose $W$ contains a sector of the form $[\vec{\kappa}(i, n) \overleftarrow{\kappa}(i, n)]$ and a sector of the form $[\overleftarrow{\kappa}(i, n) \lambda(i+1)]$. Then $\mathrm{ghg}^{-1}$ contains rules from $\mathbb{S}_{0}$.

Proof. Suppose by contradiction that $g h g^{-1}$ does not contain rules from $\mathbb{S}_{0}$. By Lemma $6.36 h$ is a word over $\mathbb{S}_{2 n+1}$.

Since $W \cdot h=W$, the $\Omega$-coordinate of $W$ is $2 n+1$.

Suppose that $g$ consists of rules from $\mathbb{S}_{2 n+1}$. Suppose that rules from $\mathbb{S}_{2 n+1}$ are active for a sector of the form $\left[z z^{\prime}\right]$ of $W$. Then $z \not \equiv \kappa(1), \rho(1)^{-1}$. Hence by Lemma 6.13 , either $g$ and $h$ commute modulo Burnside relations or $W \cdot g=W$, a contradiction. If rules from $\mathbb{S}_{2 n+1}$ are not active for sectors of $W$ then $W \cdot g=W$ by Lemma 6.4(i), a contradiction. Hence $g$ contains some rules not from $\mathbb{S}_{2 n+1}$.

Since $W$ is in the domain of $g$ (indeed $W$ is in the domain of $g h g^{-1}$ and $g h g^{-1}$ is reduced), $g$ must start with a rule from $\mathbb{S}_{2 n+1} \cup\left\{\mathbf{c}_{2 n}^{-1}\right\}$. Since $g$ contains some rules not from $\mathbb{S}_{2 n+1}, g$ has a subword of the form $\mathbf{c}_{t}^{ \pm 1} f \mathbf{c}_{t}^{\mp 1}$ where $f$ is a word over some $\mathbb{S}_{t^{\prime}}, t^{\prime} \neq 0$. By Lemma $6.11, g$ is not Burnside-reduced, a contradiction with Lemma 6.26.

Lemma 6.38. If $W$ contains an occurrence of $\vec{\kappa}(i, j, \omega)$ or $\overleftarrow{\kappa}(i, j, \omega)$ or $\lambda(i, \omega)$ then $g^{-1} g^{-1}$ contains rules from $\mathbb{S}_{0}$.

Proof. Consider the cyclic graph labeled by $\Lambda(\omega)$ where $\omega$ is the $\Omega$-coordinate of $W$. We can consider this graph as an "inverse automaton", so that we can read clockwise and counterclockwise. Of course when we pass an edge labeled by $z$ in the counterclockwise direction, we read $z^{-1}$, when we pass it in the clockwise direction, we read $z$. Then the word $z_{1} z_{2} \ldots z_{1}$ of $\mathbf{K}$-letters from $W$ can be read on this automaton, and the start edge coincides with the end edge. This interpretation makes it clear that if $W$ contains $\vec{\kappa}(i, j, \omega), \overleftarrow{\kappa}(i, j, \omega)$ or $\lambda(i, \omega)$ and does not contain sectors of the form $\left[z z^{-1}\right]$ with $z \in\left\{\vec{\kappa}(p, q, \omega), \overleftarrow{\kappa}(p, q, \omega), \overleftarrow{\kappa}(p, q, \omega)^{-1}, \lambda(p, \omega)^{-1}\right\}$ for any $p, q$ (see Lemmas $6.29,6.31,6.34)$ then $W$ must contain a sector of the form $[\vec{\kappa}(p, n) \overleftarrow{\kappa}(p, n)]$ and a sector of the form $[\overleftarrow{\kappa}(p, n) \lambda(p+1, n)]$. Now the statement of the lemma follows from Lemma 6.37 .

Lemma 6.39. $W$ does not contain sectors of the form $z w z^{-1}$ where $z \in \mathbf{K}$.

Proof. Suppose that $W$ contains such a sector. By Lemmas 6.29, 6.31, 6.33,

$$
z \in\{\lambda(i, \omega), \rho(i)\} .
$$

Case 1. Let $z \equiv \lambda(i, \omega)$. Since by Lemma $6.34 W$ does not contain sectors of the form $\left[\lambda(i)^{-1} \lambda(i)\right], W$ must contain a sector of the form $[\overleftarrow{\kappa}(i-1, n) \lambda(i)]$. Since by Lemma $6.34 W$ does not contain sectors of the form $\left[\overleftarrow{\kappa}(i-1, n)^{-1} \overleftarrow{\kappa}(i-1, n)\right], W$ must contain a sector of the form $[\vec{\kappa}(i-1, n) \overleftarrow{\kappa}(i-1, n)]$. Now by Lemma $6.37, g h g^{-1}$ contains a rule from $\mathbb{S}_{0}$. But this possibility cannot occur because rules from $\mathbb{S}_{0}$ lock $\lambda(i)$-sectors.

Case 2. Let $z \equiv \rho(i)$. Then $g h g^{-1}$ does not contain rules from $\mathbb{S}_{0}$ by Lemma 6.9. 
Suppose that $W$ contains a K-letter of the form $\vec{\kappa}(i, j, \omega), \overleftarrow{\kappa}(i, j, \omega)$ or $\lambda(i, \omega)$. Then as in the proof of Lemma 6.38, by Lemmas 6.29, 6.31, 6.34 $W$ must contain sectors of the form $[\vec{\kappa}(i, n) \overleftarrow{\kappa}(i, n)]$ and $[\overleftarrow{\kappa}(i, n) \lambda(i)]$. But this contradicts Lemma 6.37.

Therefore all K-letters in $W$ belong to the set $\left\{\kappa(i), \kappa(i)^{-1}, \rho(i), \rho(i)^{-1}\right\}$. By Lemma 6.32 not all K-letters belong to the set $\left\{\rho(i), \rho(i)^{-1}\right\}$. By Lemma 6.33, not all of them belong to $\left\{\kappa(i), \kappa(i)^{-1}\right\}$. Therefore for some $i$ from 1 to $N$ each sector of $W$ has one of the four forms $[\kappa(i) \rho(i)],\left[\kappa(i)^{-1} \kappa(i)\right]$, $\left[\rho(i) \rho(i)^{-1}\right],\left[\rho(i)^{-1} \rho(i)\right]$ (recall that sectors of the form $\left[\kappa(i) \kappa(i)^{-1}\right]$ cannot appear by Lemma 6.33). Moreover a sector of the form $\left[\kappa(i)^{-1} \kappa(i)\right]$ must appear among sectors of $W$ (the $\mathbf{K}$-letter preceding $\kappa(i)$ in $W$ cannot be $\lambda(i, \omega)$ since $W$ does not contain letters of the form $\lambda(j, \omega))$.

If rules from $\mathbb{S}_{2 n+1}$ do not appear in $g, h$ then we can consider any sector of $W$ of the form $\left[\kappa(i)^{-1} \kappa(i)\right]$, and use Lemma 6.10 to get a contradiction. Therefore rules from $\mathbb{S}_{2 n+1}$ do appear in $g h g^{-1}$. By the same reason not all rules in $g h g^{-1}$ are from $\mathbb{S}_{2 n+1}$. Therefore $g h g^{-1}$ contains $\mathbf{c}_{2 n}$. Since $\mathbf{c}_{2 n}$ locks $\kappa(i)$-sectors, sectors of the form $\left[\rho(i)^{-1} \rho(i)\right]$ do not appear in $W$. Therefore for some $i$ all sectors of $W$ have the form $\left[\kappa(i)^{-1} \kappa(i)\right]$, $[\kappa(i) \rho(i)]$ or $\left[\rho(i) \rho(i)^{-1}\right]$. But this contradicts the assumption of part (ii) of Proposition 6.17.

Recall that $W$ is an admissible word which starts and ends with the same letter and does not contain accepted subwords. By replacing $W$ with $W^{-1}$ if necessary we can assume that the first letter in $W$ belongs to $\overline{\mathbf{K}}(\omega)$ for some $\omega \in \Omega$. Then by Lemma 6.39 all $\mathbf{K}$-letters in $W$ belong to $\overline{\mathbf{K}}(\omega)$. Since $W$ is an admissible word, it must contain an admissible subword $W^{\prime}$ whose projections onto K-letters is equal to a cyclic shift of $\Lambda(\omega)$.

Lemma 6.40. $W^{\prime}$ is an accepted word.

Proof. By definition, $W$ contains sectors of the form $\left[z z_{+}\right]$for every $z \in \overline{\mathbf{K}}(\omega)$. In particular, $W$ contains sectors of the form $[\vec{\kappa}(1, n) \overleftarrow{\kappa}(1, n)]$ and $[\overleftarrow{\kappa}(1, n) \lambda(1, n)]$. By Lemma $6.37, g h g^{-1}$ contains a rule from $\mathbb{S}_{0}$. By Lemma $6.13, g h g^{-1}$ contains rules from at least two different $\mathbb{S}_{i}$. Hence $g h g^{-1}$ also contains either rules from $\mathbf{S}_{1}$ or $\mathbf{c}_{0}^{ \pm 1}$.

Let us prove that $g h g^{-1}$ or $g h^{-1} g^{-1}$ contains a subword $\mathbf{c}_{0} h_{1} c_{1}$ where $h_{1}$ is a word over $\mathbf{S}_{1}$. Suppose that it is not true. Then $g h g^{-1}$ contains rules from $\mathbb{S}_{0} \cup\left\{\mathbf{c}_{0}, \mathbf{c}_{0}^{-1}\right\} \cup \mathbb{S}_{1}$ only. Hence $g h g^{-1}$ contains $\mathbf{c}_{0}$ and $\mathbf{c}_{0}^{-1}$. Since the $\Omega$-coordinates of $W, W \cdot g, W \cdot g h$ are the same, the total exponent of $\mathbf{c}_{0}$ in $g$ is 0 and the total exponent of $\mathbf{c}_{0}$ in $h$ is 0 . If $g$ or $h$ contains a subword $\mathbf{c}_{0} f \mathbf{c}_{0}^{-1}$ where $f$ is a word over $\mathbb{S}_{1}$, then by Lemma 6.7 , applied to any $\overleftarrow{\kappa}(i, j)$-sector of $W$, we get that $f=1$ modulo Burnside relations, a contradiction with Lemma 6.26. Hence neither $g$ nor $h$ contain subwords of the form $\mathbf{c}_{0} f \mathbf{c}_{0}^{-1}$. Now consider two cases.

Case 1. Suppose that $\omega=0$. Since $W \cdot g h g^{-1}=W$, the first occurrence of $\mathbf{c}_{0}^{ \pm 1}$ in $g h g^{-1}$ is $\mathbf{c}_{0}$. Since $h$ does not contain subwords of the form $\mathbf{c}_{0} f \mathbf{c}_{0}^{-1}$, this $\mathbf{c}_{0}$ occurs in $g$. But since $W \cdot g$ has the same $\Omega$-coordinate as $W, g$ must contain $\mathbf{c}_{0}^{-1}$, hence $g$ contains a subword of the form $\mathbf{c}_{0} f \mathbf{c}_{0}^{-1}$, a contradiction.

Case 2. Suppose that $\omega=1$. Then the first occurrence of $\mathbf{c}_{0}^{ \pm 1}$ in $g h g^{-1}$ is $\mathbf{c}_{0}^{-1}$. Since $W \cdot g=W$, we can represent $g$ as $g_{1} g_{2}$ where $g_{2}$ is the maximal suffix of $g$ consisting of rules from $\mathbb{S}_{1}$ and $g_{1}$ is either empty or ends with $\mathbf{c}_{0}$. Since $W \cdot h=W$, we can represent $h$ as $h_{1} h_{2} h_{3}$ where $h_{1}$ and $h_{3}$ consist of rules from $\mathbb{S}_{1}$ and either $h_{2}=h_{3}$ is empty or $h_{2}$ starts with $\mathbf{c}_{0}^{-1}$ and ends with $\mathbf{c}_{0}$. Consider any $\overleftarrow{\kappa}(1,1)$-sector $U \equiv \overleftarrow{\kappa}(1,1,1) u \vec{\kappa}(1,2,1)$ of $W$.

Case 2.1. Suppose that $g_{1}$ is empty, $g \equiv g_{2}$ consists of rules from $\mathbb{S}_{1}$. Since $g h g^{-1}$ contains $\mathbf{c}_{0}$, $h$ must contain $\mathbf{c}_{0}$, so $h_{2}$ is not empty. 
Since $\mathbf{c}_{0}$ locks $U$ and rules from $g$ are left active for $\overleftarrow{\kappa}(1,1)$-sectors, by Lemma 6.4 (i), we have $R^{a}\left(g h_{1}\right)=u=\left(R^{a}\left(h_{3} g^{-1}\right)\right)^{-1}$ modulo Burnside relations. By Lemma 6.4 (iii), we have that $g h_{1}=g h_{3}^{-1}$ modulo Burnside relations. Hence $h_{1}=h_{3}^{-1}$ modulo Burnside relations. This contradicts Lemma 6.27 if $h_{1}$ or $h_{3}$ is not empty or Lemma 6.24 if $h_{1}, h_{3}$ are empty.

Case 2.2. Suppose that $g_{1}$ is not empty. Then $g_{1}$ ends with $\mathbf{c}_{0}^{-1}$. If $h$ does not contain $\mathbf{c}_{0}$, that is $h_{2}, h_{3}$ are empty, then by Lemma 6.7 applied to a $\vec{\kappa}(1,1)$-sector of $W, g_{2} h g_{2}^{-1}=1$ modulo Burnside relations, so $h=1$ modulo Burnside relations, a contradiction.

If $h$ contains $\mathbf{c}_{0}$, that is $h_{2}$ is not empty, then again by Lemma $6.7 g_{2} h_{1}=1, h_{3} g_{2}^{-1}=1$ modulo Burnside relations. Thus $h_{1}=h_{3}^{-1}$ modulo Burnside relations which as before contradicts Lemma 6.27 or Lemma 6.24 .

Thus, since one can always consider $h^{-1}$ instead of $h$, we can assume that $h$ has the form $e \mathbf{c}_{0} h_{1} \mathbf{c}_{1} f$ for some words $e, f$. Since $\mathbf{c}_{0}$ locks $z$-sectors, $z \in\{\lambda(i), \kappa(i), \rho(i), \overleftarrow{\kappa}(i, j)\}$, a cyclic shift of $W^{\prime} \cdot e$ has the form

$$
\lambda(1,0) \kappa(1) \rho(1) \vec{\kappa}(1,1,0) u_{1,1} \overleftarrow{\kappa}(1,1,0) \vec{\kappa}(1,2,0) u_{1,2} \overleftarrow{\kappa}(1,2,0) \ldots \vec{\kappa}(N, n, 0) u_{N, n} \overleftarrow{\kappa}(N, n, 0)
$$

The word $W^{\prime} \cdot e \mathbf{c}_{0}$ has the form

$$
\lambda(1,1) \kappa(1) \rho(1) \vec{\kappa}(1,1,1) u_{1,1} \overleftarrow{\kappa}(1,1,1) \vec{\kappa}(1,2,1) u_{1,2} \overleftarrow{\kappa}(1,2,1) \ldots \vec{\kappa}(N, n, 1) u_{N, n} \overleftarrow{\kappa}(N, n, 1)
$$

Since $\mathbf{c}_{1}$ locks $\vec{\kappa}(i, j)$-sectors, for every $i \in\{1, \ldots, N\}$ and every $j \in\{1, \ldots, n\}$, we have

$$
R^{a}\left(h_{1}, \vec{\kappa}(i, j)\right)=u_{i, j}
$$

modulo Burnside relations. Hence all $u_{i, j}$ are equal modulo Burnside relations, so a cyclic shift of $W^{\prime} \cdot e$ is equal to the word

$$
\lambda(1,0) \kappa(1,0) \rho(1,0) \vec{\kappa}(1,1,0) u \overleftarrow{\kappa}(1,1,0) \vec{\kappa}(1,2,0) u \overleftarrow{\kappa}(1,2,0) \ldots \vec{\kappa}(N, n, 0) u \overleftarrow{\kappa}(N, n, 0)
$$

for some word $u$ which is equal to $u_{i, j}$ modulo Burnside relations. Denote by $\bar{u}$ the copy of $u$ written in $\mathbb{S}_{0}$. Then by Lemma $6.4 W^{\prime} \cdot e \bar{u}=\Lambda(0)$, so $W^{\prime}$ is accepted.

Lemma 6.40 gives us the final contradiction because by our assumption $W$ cannot contain an accepted subword. Proposition 6.17 is proved.

\section{Bands, annuli, and trapezia}

\subsection{Definitions and basic facts}

In Section 6, we introduced an auxiliary group $H_{k a}^{*}$ which was the free product of the subgroup $\langle\mathbf{K}\rangle$ freely generated by $\mathbf{K}$ and the subgroup $H_{a}$ generated by the set $\mathbf{A}$ subject to the $R(\kappa(1))$ relations and the Burnside relations. Recall that the subgroup $H_{a}$ is the free product in the variety of Burnside groups of exponent $n$ of its subgroups $\langle A(z)\rangle, z \in \overline{\mathbf{K}}$. From now on let us call all relations of $H_{a}$ as a-relations. All relations from $Z(\mathbb{S})$ involving letters from $\mathbf{K}$ will be called $\kappa$-relations and all relations involving letters from $\mathbf{R}$ will be called $r$-relations. We also will call 
the commutativity relations from $Z(\mathbb{S})$ involving $\mathbf{R}$ - and A-letters ra-relations. Accordingly we shall consider $a$-cells, $\kappa$-cells, $r$-cells and $r a$-cells in van Kampen diagrams. Notice that $\kappa$-cells are also $r$-cells. Recall that the set of relations $Z(\mathbb{S}, \Lambda)$ consists of $Z(\mathbb{S})$ and the hub $\Lambda(0)$. The cells corresponding the hub relation, will be called hubs as well.

In this section, we shall consider diagrams over the set $Z(\mathbb{S}, a)$ of all $a$-relations and all $r$-relations (including $\kappa$-relations).

With every word over $\mathbf{K} \cup \mathbf{R} \cup \mathbf{A}$ we associate its $\mathbf{K}$-length, its $\mathbf{R}$-length and its $\mathbf{A}$-length in the natural way. In any diagram over $Z(\mathbb{S}, a)$ we consider $\mathbf{R}$-edges and $\mathbf{A}$-edges as 0-edges and $\mathbf{K}$-edges as non-zero edges. In a diagram is over the presentation consisting of $a$-relations and $r a$-relations, then we consider $\mathbf{R}$-edges as non-zero edges and $\mathbf{A}$-edges as zero edges. Thus the metric on a diagram depends on over what presentation this diagram is.

Let $S$ be one of the sets $\mathbf{K}, \mathbf{R}, \mathbf{A}$. Then we can consider $S$-bands in any diagram over $Z(\mathbb{S}, a)$ as in Section 2.2 .

- K-bands consist of $\kappa$-cells. A maximal $\mathbf{K}$-band which is not an annulus can start and end on the boundary of a diagram. All $\mathbf{K}$-letters of a $\mathbf{K}$-band belong to the same set $\overline{\mathbf{K}}(z)$ for some $z \in \overline{\mathbf{K}} \cup \overline{\mathbf{K}}^{-1}$. Thus we can consider $\overline{\mathbf{K}}(z)$-bands as well.

- $\mathbf{R}$-bands consist of $r$-cells. A maximal $\mathbf{R}$-band which is not an annulus must start and end on the boundary of the diagram. For every $\mathbf{R}$-band $\mathcal{T}$ there exists a rule $\tau \in \mathbb{S}$ such that $\mathbf{R}$-edges in an $\mathbf{R}$-band have labels of the form $r(\tau, z), z \in \overline{\mathbf{K}}$. Thus we will sometimes call $\mathcal{T}$ a $\tau$-band.

The next lemma immediately follows from the definition of words $L(., z)$ and $R(., z)$ and the definition of $\overline{\mathbf{K}}$-relations.

Lemma 7.1. Consider a disc or an annular diagram consisting of one $\overline{\mathbf{K}}(z)$-band $\mathcal{T}$. Then for some word $h$ over $\mathbb{S}$ (which is called the history of the band), the word written on the top of $\mathcal{T}$ is $R(h, z)$, the word written on the bottom of $\mathcal{T}$ is $L(h, z)$. The word $R(h, z)$ (resp. $L(h, z)$ ) is $\mathbf{R}$-reduced if and only if $\mathcal{T}$ does not contain two consecutive cells that cancel. Moreover $L(h, z) z^{\prime}=$ $z R(h, z)$ modulo $\kappa$-relations, where $z, z^{\prime}$ are the labels of the start and end edges of the band.

The next lemma contains easy properties of $\mathbf{R}$-bands which follow immediately from the definition of $Z(\mathbb{S})$ and Lemma 5.1.

Lemma 7.2. Let

$$
w \equiv u_{0} z_{1} u_{1} z_{2} \ldots z_{s} u_{s}
$$

be the bottom side of a $\tau$-band $\mathcal{T}$,

$$
w^{\prime} \equiv u_{0}^{\prime} z_{1}^{\prime} u_{1}^{\prime} z_{2}^{\prime} \ldots z_{s^{\prime}}^{\prime} u_{s^{\prime}}^{\prime}
$$

be the top side of $\mathcal{T}$, where $z_{i}, z_{i}^{\prime} \in \overline{\mathbf{K}} \cup(\overline{\mathbf{K}})^{-1} u_{i}, u_{i}^{\prime}$ are words over $\mathbf{A}$. Then $W \equiv z_{1} u_{1} z_{2} \ldots z_{s}$ and $W^{\prime} \equiv z_{1}^{\prime} u_{1}^{\prime} z_{2}^{\prime} \ldots z_{s^{\prime}}^{\prime}$ are admissible words and $W \cdot \tau=W^{\prime}$. 


\subsection{Forbidden annuli}

Let $\Delta$ be any (disc or annular) diagram over $Z(\mathbb{S}, a)$. We call $\Delta Z(\mathbb{S}, a)$-reduced if every $\mathbf{K}$-band is reduced (that is they do not contain consecutive $\mathbf{K}$-cells that cancel) and every disc subdiagram whose boundary label is a word over $\mathbf{A}$ and is equal to 1 modulo $a$-relations does not contain R-edges.

Lemma 7.3. Let $\Delta$ be a $Z(\mathbb{S}, a)$-reduced disc diagram. Then $\Delta$ does not contain

1. K-annuli,

2. (K, R)-annuli,

3. $\mathbf{R}$-annuli,

4. disc subdiagrams with some r-cells whose boundary path contains no $\mathbf{R}$-edges,

Proof. We are proving these statements by a joint induction on the number of cells of the inside diagrams of the annulus or the "bad" subdiagrams. By contradiction take the smallest (with respect to the number of cells) disc subdiagram $\Delta^{\prime}$ of $\Delta$ which either contains one of the annuli forbidden by the lemma or has boundary path forbidden by the lemma.

Case 1. Assume there is a $\mathbf{K}$-annulus $\mathcal{Q}$ in $\Delta$. Then there exists an $\mathbf{R}$-edge on a side of $\mathcal{Q}$. Hence $\mathcal{Q}$ crosses an $\mathbf{R}$-band $\mathcal{T}$. Since $\mathcal{Q}$ and $\mathcal{T}$ form a $(\mathbf{K}, \mathbf{R})$-annulus with the inside diagram smaller than that for $\mathcal{Q}$, we obtain a contradiction.

Case 2. Suppose that $\Delta^{\prime \prime}$ is the inside diagram of a $(\mathbf{K}, \mathbf{R})$-annulus $\mathcal{W}_{1} \cup \mathcal{W}_{2}$ where $\mathcal{W}_{1}$ is a $\mathbf{K}$-band and $\mathcal{W}_{2}$ is an $\mathbf{R}$-band.

Then the contour of $\Delta^{\prime \prime}$ does not contain $\overline{\mathbf{K}}$-edges (otherwise there would be a $(\mathbf{K}, \mathbf{R})$-annulus with a smaller inside diagram). Therefore $\mathcal{W}_{1}$ consists of two cells. These two cells have a common $\mathbf{K}$-edge and they belong to the same $r$-band $\mathcal{W}_{2}$. Therefore these two $\kappa$-cells cancel, a contradiction.

Case 3. Suppose that there is a $\kappa$-cell in an $\mathbf{R}$-annulus $\mathcal{Q}$. Then we get a contradiction as in Case 1. Therefore the boundary label $W$ of $\Delta^{\prime \prime}$ is a word in A. Since the counterexample is minimal, $\Delta^{\prime \prime}$ has no edges except for A-edges. Hence $W=1$ modulo $a$-relations. Diagram $\Delta^{\prime}$ has the same boundary label $W$, since all $(r, a)$-cells in $\mathcal{Q}$, are commutativity cells. But this contradicts the property that $\Delta^{\prime}$ is $Z(\mathbf{S}, \mathbf{a})$-reduced, because there are $\mathbf{R}$-edges in $\mathcal{Q}$.

Case 4. Suppose that $\partial\left(\Delta^{\prime}\right)$ contains no R-edges. Then by part 3 of the lemma, $\Delta^{\prime}$ contains no $\mathbf{R}$-edges, so it cannot contain $r$-cells, a contradiction.

Corollary 7.4. The group $H_{k a}^{*}$ is (naturally) embedded into the group defined by $Z(\mathbb{S}, a)$.

Proof. Indeed, let $W$ be a word in $\mathbf{K} \cup \mathbf{A}$ which is equal to 1 modulo $Z(\mathbb{S}, a)$. Then there exists a $Z(\mathbb{S}, a)$-reduced van Kampen disc diagram $\Delta$ with boundary label $W$. By Lemma 7.3, part $4, \Delta$ contains no $r$-cells, so all cells in $\Delta$ are $a$-cells. Hence $W=1$ modulo $a$-relations, so $W=1$ in $H_{k a}^{*}$. Thus the natural map of $H_{k a}^{*}$ into the group given by the relations $Z(\mathbb{S}, a)$ is an embedding. 


\subsection{Trapezia}

Here we shall present a tool to translate results from Section 6 into the language of van Kampen diagrams and vice versa.

Let $\Delta$ be a $Z(\mathbb{S}, a)$-reduced disc diagram which has the contour of the form $p_{1} q_{1} p_{2}^{-1} q_{2}^{-1}$ where:

(TR1) $\phi\left(q_{1}\right), \phi\left(q_{2}\right)$ are K-reduced words without R-letters, starting and ending with K-letters, and

(TR2) $p_{1}$ and $p_{2}$ are sides of reduced $\mathbf{K}$-bands each containing at least one $\kappa$-cell.

Then $\Delta$ is called a trapezium. The path $q_{1}$ is called the bottom base, the path $q_{2}$ is called the top base of the trapezium, the paths $p_{1}$ and $p_{2}$ are called the left and right sides of the trapezium. The history of the $\mathbf{K}$-band whose side is $p_{1}$ is called the history of the trapezium.

By Lemma 5.1, if a word $h$ over $\mathbb{S}$ contains just one rule $\tau, W \equiv z_{1} u_{1} \ldots z_{t}$ is a reduced admissible word, and $W \cdot h=W_{1}$, then there exists a word $W^{\prime}$ which is equal to $W$ modulo $a$-relations and such that $\tau$ is applicable to $W^{\prime}, W^{\prime} \cdot h=W_{1}^{\prime}$ where $W_{1}^{\prime}$ is equal to $W_{1}$ modulo $a$-relations. By Lemma 5.1 there exists a trapezium $\Theta$ consisting of one $\tau$-band with bottom base label $W^{\prime}$, top base label $W_{1}^{\prime}$. Since $W=W^{\prime}, W_{1}=W_{1}^{\prime}$ modulo Burnside relations, there exist diagrams $\Delta, \Delta_{1}$ consisting of a-cells with boundary labels $W\left(W^{\prime}\right)^{-1}$ and $W_{1}\left(W_{1}^{\prime}\right)^{-1}$ respectively. Gluing three diagrams $\Delta, \Theta$, $\Delta_{1}$ in a natural way, we get a trapezium $T(W, \tau)$ with bottom base label $W$, top base label $W_{1}$, left side label $L\left(\tau, z_{1}\right)$, right side label $R\left(\tau, z_{t}\right)$.

If a reduced word $h$ consists of several rules, the corresponding trapezium can be obtained by induction. Let $h=h^{\prime} \tau$ where $h^{\prime}$ is a word of smaller length than $h$. Suppose that we have constructed a trapezia $T\left(W, h^{\prime}\right)$ with the bottom base label $W$ and the top base label $W \cdot h^{\prime}$, and suppose that $W \cdot h$ exists. Then $W \cdot h^{\prime}$ is in the domain of $\tau$, so $\tau$ is applicable to a reduced admissible word $W_{1}$ which is equal to $W \cdot h^{\prime}$ modulo $a$-relations. Then there exists a diagram $\Delta$ over the $a$-relations with contour $s_{1} s_{2}^{-1}$ where $\phi\left(s_{1}\right)=W \cdot h^{\prime}, \phi\left(s_{2}\right)=W_{1}$. By Lemma 5.1 there exists a trapezium $T\left(W_{1}, \tau\right)$ with the bottom base label $W_{1}$. Glue $T\left(W, h^{\prime}\right)$ with the diagram $\Delta$ identifying the top base of $T\left(W, h^{\prime}\right)$ with $s_{1}$. Then glue the resulting diagram with $T\left(W_{1}, \tau\right)$ identifying $s_{2}$ with the bottom base of $T\left(W_{1}, \tau\right)$. The $\mathbf{K}$-bands in the resulting diagram $T(W, h)$ are reduced because $h$ is a reduced word. Every closed path in $T(W, h)$ consisting of A-edges is contained in one of the subdiagrams $\Delta$ consisting of $a$-cells. Hence $T(W, h)$ is a $Z(\mathbb{S}, a)$-reduced diagram. It is clear that conditions (TR1) and (TR2) are satisfied. Hence $T(W, h)$ is a trapezium with bottom base label $W$, top base label $W \cdot h$, left side label $L\left(h, z_{1}\right)$ and right side label $R\left(h, z_{t}\right)$. It has a form of a sandwich where diagrams consisting of $a$-cells are sandwiched between $\tau$-bands (see Figure 14).

Clearly $T(W, h)$ is not determined uniquely by $W$ and $h$ because we can choose the $a$-parts of the sandwich in many different ways. Any trapezium of the form $T(W, h)$ is called a normal trapezium with history $h$ and base label $W$. 


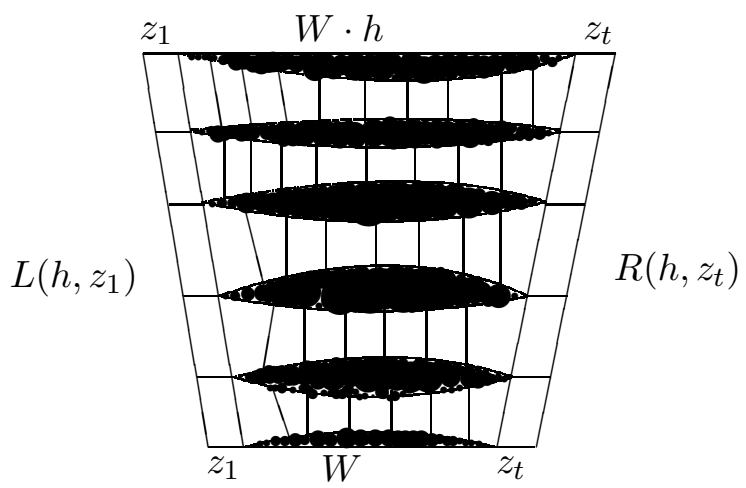

Fig. 14 .

Lemma 7.5. Every trapezium is a normal trapezium $T(W, h)$ for some $W$ and some $h$.

Proof. Consider a trapezium $\Delta$ with bases $q_{1}, q_{2}$ and sides $p_{1}, p_{2}$. By condition (TR2) $p_{1}$ and $p_{2}$ contain R-edges. Since by (TR2), $p_{1}$ and $p_{2}$ are sides of reduced $\mathbf{K}$-bands, $\phi\left(p_{1}\right), \phi\left(p_{2}\right)$ are $\mathbf{R}$ reduced. Since $\Delta$ is $Z(\mathbb{S}, a)$-reduced, we can apply Lemma 7.3(2) and conclude that every maximal $\mathbf{R}$-band starting on $p_{1}$ ends on $p_{2}$ and every maximal $\mathbf{R}$-band starting on $p_{2}$ ends on $p_{1}$. By Lemma 7.3(3), $\Delta$ does not contain any other maximal $\mathbf{R}$-bands.

Since by (TR1) $\phi\left(q_{1}\right)$ and $\phi\left(q_{2}\right)$ are $\mathbf{K}$-reduced, by Lemma 3.2 every maximal K-band starting on $q_{1}$ (resp. $q_{2}$ ) ends on $q_{2}$ (resp. $q_{1}$ ) and by Lemma 7.3(1) $\Delta$ does not have any other maximal K-bands.

Let $\mathcal{T}, \mathcal{T}^{\prime}$ be the first and the last maximal $\mathbf{K}$-bands in $\Delta$ starting on $q_{1}$ counting from $p_{1}$ to $p_{2}$. Let $\mathcal{B}$ and $\mathcal{B}^{\prime}$ be the first and the last $\mathbf{R}$-bands of $\Delta$, counting from $q_{1}$ to $q_{2}$.

Consider the subdiagram $\Delta^{\prime}$ of $\Delta$ bounded by $q_{1}$ and the connecting line of $\mathcal{B}$. By Lemma 7.2 the boundary label of $\Delta^{\prime}$ is equal to $\phi\left(q_{1}\right) W^{-1}$ where $W$ is an admissible word. Since $\Delta^{\prime}$ does not contain $r$-cells (by Lemma 7.3, part 3), $W=\phi\left(q_{1}\right)$ in $H_{k a}^{*}$. Since $\phi\left(q_{1}\right)$ is reduced by (TR1), $W$ is a reduced admissible word and $\phi\left(q_{1}\right)$ is a reduced admissible word which is equal to $W$. Let $\Delta^{\prime \prime}$ be the diagram obtained from $\Delta$ by removing $\Delta^{\prime}$ and $\mathcal{B}$. Then $\partial\left(\Delta^{\prime \prime}\right)=p_{1}^{\prime} q_{1}^{\prime}\left(p_{2}^{\prime}\right)^{-1}\left(q_{2}^{\prime}\right)^{-1}$ where $p_{1}^{\prime}$ is a side of $\mathcal{T}$ without the first cell, $p_{2}^{\prime}$ is a side of $\mathcal{T}^{\prime}$ without the first cell, $q_{1}^{\prime}$ is a side of $\mathcal{B}, q_{2}^{\prime}=q_{2}$. Since by Lemma $7.2 \phi\left(q_{1}^{\prime}\right)=\phi\left(q_{1}\right) \cdot \tau$ in $H_{k a}^{*}$ if $\mathcal{B}$ is a $\tau$-band, we have that $\phi\left(q_{1}^{\prime}\right)$ is $\mathbf{K}$-reduced.

Suppose that $p_{1}^{\prime}$ does not have $\mathbf{R}$-edges. Then $p_{2}^{\prime}$ does not have $\mathbf{R}$-edges either (every maximal $\mathbf{R}$-band of $\Delta^{\prime \prime}$ is a maximal $\mathbf{R}$-band of $\Delta$ ), so the boundary of $\Delta^{\prime \prime}$ contains no $\mathbf{R}$-edges. By Lemma 7.3 (4) $\Delta^{\prime \prime}$ does not contain $r$-cells, hence $\phi\left(q_{2}\right)=\phi\left(q_{1}^{\prime}\right)=\phi\left(q_{1}\right) \cdot \tau$ in $H_{k a}^{*}$, hence $\Delta=T\left(\phi\left(q_{1}\right), \tau\right)$ as required.

Now if $p_{1}^{\prime}$ contains $\mathbf{R}$-edges then $p_{2}^{\prime}$ also contains $\mathbf{R}$-edges, so $\Delta^{\prime \prime}$ satisfies conditions (TR1) and (TR2), so $\Delta^{\prime \prime}$ is a trapezium. Since $\Delta^{\prime \prime}$ contains fewer maximal $\mathbf{R}$-bands than $\Delta$, by induction, we can assume that $\Delta^{\prime \prime}=T\left(\phi\left(q_{1}^{\prime}\right), h\right)$ for some $h$. Then $\Delta=T\left(\phi\left(q_{1}\right), \tau h\right)$ as required

\section{The group $H_{r a}$}

Consider the group $H_{r a}$ which is the group generated by $\mathbf{A} \cup \mathbf{R}$ subject to the set of relations consisted of all $a$-relations and all (commutativity) ra-relations from $Z(\mathbb{S}, a)$. We consider A- 
letters as zero-letters and R-letters as non-zero letters. So in terminology of Section 3, in this section $Y=\mathbf{R}$.

We are going to prove that the presentation of $H_{r a}$ satisfies conditions (Z1), (Z2), (Z3) from Section 3

Locally, in this section, we are going to keep the same notation as in Section 3 . We let $\mathcal{R}_{0}=\mathcal{S}_{0}$ be the set of $a$-relations and ra-relations. The set $\mathcal{S}_{1 / 2}$ is thus empty. Clearly conditions (Z1.1) and (Z1.2) hold.

Since $\mathcal{S}_{1 / 2}$ is empty, conditions (Z2.1), (Z2.2) and (Z2.3) also hold.

We will use the terminology and results from Section 3.1 and Section 3.2. In particular, we are going to talk about essential, reduced and cyclically reduced words in $H_{r a}$.

As in Section 3, for every cyclically reduced essential element $g \in H_{r a}$ we define $\mathbf{0}(g)=\mathbf{0}(g)$ as the maximal subgroup of $H_{a}$ which contains $g$ in its normalizer. For an arbitrary essential element $g$ of the form $v u v^{-1}$ where $u$ is cyclically reduced, we define $\mathbf{0}(g)=v \mathbf{0}(u) v^{-1}$. By Lemma 3.7, $\mathbf{0}(g)$ is well defined (does not depend on the presentation $g=v u v^{-1}$ ).

The following lemma will imply both (Z3.1) and (Z3.2).

Lemma 8.1. Let $g$ be an essential element represented by a cyclically reduced word $A$, let $x \neq 1$ be a 0-element such that $g^{-4} \mathrm{xg}^{4}$ is a 0-element. Then the following two statements hold.

(i) There exists a 0 -element $u$ such that $g u^{-1}$ commutes with every element of $\mathbf{0}(g)$.

(ii) $x \in \mathbf{0}(g)$.

Proof. By Lemma 3.6, $g^{-2} x g^{2}$ is 0-element. Let $B$ be a word in $\mathbf{A}$ representing a non-trivial element $x$ from $\mathbf{0}(g), C$ be a word in $\mathbf{A}$ representing $g^{-2} x g^{2}$.

Let $A \equiv u_{1} r_{1} u_{2} \ldots r_{s} u_{s+1}$ where $r_{i} \in \mathbf{R}, i=1, \ldots, s, u_{i}$ are words in $\mathbf{A}, i=1, \ldots, s+1$. By Lemma 3.7 (all of the conditions of that lemma hold), we can replace $A$ by any cyclic shift of $A$. So we can assume that $u_{1}$ is empty.

Then there exists a diagram $\Delta$ of minimal type over the presentation $\mathcal{S}_{0}$ of $H_{r a}$ with boundary $p_{1} q_{1} p_{2}^{-1} q_{2}^{-1}$ where $\phi\left(q_{1}\right) \equiv \phi\left(q_{2}\right) \equiv A^{2}, \phi\left(p_{1}\right) \equiv B, \phi\left(p_{2}\right) \equiv C$.

Since $A$ is cyclically reduced and $p_{1}, p_{2}$ do not contain $\mathbf{R}$-edges, every maximal $\mathbf{R}$-band in $\Delta$ starting on $q_{1}$ ends on $q_{2}$ and every maximal R-band starting on $q_{2}$ ends on $q_{1}$. By Lemma 7.3 (3), $\Delta$ contains no other maximal $\mathbf{R}$-bands.

Let $\mathcal{T}_{1}, \ldots, \mathcal{T}_{2 s}$ be all maximal $\mathbf{R}$-bands starting on $q_{1}$, counted from $p_{1}$ to $p_{2}$. Notice that every $r a$-relation is a commutativity relation of the form $r a=a r$ where $r \in \mathbf{R}(z), a \in A(z)$ for some $z \in \overline{\mathbf{K}}$. For every $i=1, \ldots, 2 s, r_{i}$ belongs to $\mathbf{R}\left(z_{i}\right)$ for some $z_{i} \in \overline{\mathbf{K}}$. Then the labels of the top and the bottom paths of $\mathcal{T}_{i}$ are equal to a word $v_{i}$ in the alphabet $A\left(z_{i}\right) \cup A\left(z_{i}\right)^{-1}$. Notice that $B$ is conjugate of each of the words $v_{i}, i=1, \ldots, 2 s$ in $H_{r a}$. Indeed, the subdiagram of $\Delta$ bounded by $p_{1}, q_{1}, q_{2}$ and the connecting line of $\mathcal{T}_{i}$ has boundary label $t^{-1} B t v_{i}^{-1}$ for some word $t$. Since $B \neq 1$ modulo in $H_{r a}$, none of the words $v_{i}$ is equal to 1 modulo in $H_{r a}$.

The connecting lines of the bands $\mathcal{T}_{1}, \ldots, \mathcal{T}_{2 s}$ divide $\Delta$ into diagrams $\Delta_{0}, \ldots, \Delta_{2 s+1}$ such that the boundary of each $\Delta_{i}$ contains no R-edges. By Lemma 7.3 (4), each $\Delta_{i}$ has no $r$-cells, so the boundary label of each $\Delta_{i}$ is equal to 1 in the group $H_{a}$.

Since $A$ starts with $r_{1}$, the subdiagram $\Delta_{0}$ is bounded by a side of $\mathcal{T}_{1}$ and $p_{1}$. Hence $B=$ $\phi\left(p_{1}\right)=v_{1}$ modulo $a$-relations.

The boundary label of $\Delta_{1}$ is $u_{2}^{-1} v_{1} u_{2} v_{2}^{-1}$. Hence $u_{2}^{-1} v_{1} u_{2}=v_{2}$ in $H_{a}$. Recall that $H_{a}$ is the free product of subgroups $\langle A(z)\rangle$ in the variety of Burnside groups of exponent $n$. By Lemma 36.2, 34.11 from [30], each of these subgroups is malnormal in $H_{a}$. Therefore $v_{2}, u_{2} \in\left\langle A\left(z_{1}\right)\right\rangle$ modulo 
$a$-relations. Since $u_{2}, v_{2} \neq 1, z_{1} \equiv z_{2}$. Now considering subdiagrams $\Delta_{2}, \ldots, \Delta_{s+1}$ one by one, we conclude that $u_{i}, v_{i}$ are words in $A\left(z_{1}\right), i=1, \ldots, s+1$, and $z_{1}=z_{2}=\ldots=z_{s}$. Therefore all $r_{i}$ belong to $\mathbf{R}\left(z_{1}\right)$. Since $v_{i}$ are not empty, $r_{i}$ commutes with some letters from $A\left(z_{i}\right)$. By definition of $Z(\mathbb{S})$ then $r_{i}$ commutes with every letter from $A\left(z_{1}\right)$ in $H_{r a}$. Hence $r_{1}, \ldots, r_{s}$ commute with all letters from $u_{1}, \ldots, u_{s+1}$. In particular, $A=r_{1} r_{2} \ldots r_{s} u_{1} \ldots u_{s+1}$ in $H_{r a}$.

Let $u=u_{1} \ldots u_{s}$. This word does not contain letters from $\mathbf{R}$. Notice that $H_{a}$ is a retract of $H_{r a}, u$ is the $H_{a}$-projection of $g$, so $u$ does not depend in $H_{r a}$ on the word $A$ representing $g$. Let also $g_{1}=r_{1} \ldots r_{s}$. Since $\langle\mathbf{R}\rangle$ is a retract of $H_{r a}, g_{1}$ does not depend on the word $A$ representing $g$, $g u^{-1}=g_{1}$.

We have proved that $B$ is equal modulo $a$-relations to a word in $A\left(z_{1}\right)$. So $g_{1}$ commutes with $B=x$. Since $\mathbf{0}(g)$ is a 0 -subgroup normalized by $g$, it consists of 0 -elements $y$ such that $g^{-2} y g^{2}$ is a 0 -element. We have proved that $g_{1}$ commutes with every such $y$. Hence $g u^{-1}$ commutes with all elements in $\mathbf{0}(g)$ which proves (i).

Notice that the subgroup $\left\langle A\left(z_{1}\right)\right\rangle$ is normalized by $g=g_{1} u$ because $g_{1}$ commutes with this subgroup and $u$ belongs to it. Hence $\left\langle A\left(z_{1}\right)\right\rangle \subseteq \mathbf{0}(g)$. Since $x \in\left\langle A\left(z_{1}\right)\right\rangle, x \in \mathbf{0}(g)$, which proves (ii).

We have checked all properties (Z1), (Z2), (Z3), so by Proposition 3.19, we have the following statement.

Proposition 8.2. There exists a graded presentation $\mathcal{R}_{\text {ra }}$ of the factor group $H_{r a}(\infty)$ of $H_{\text {ra }}$ over the subgroup generated by all $n$-th powers of elements of $H_{r a}$, such that every g-reduced diagram over $\mathcal{R}_{\text {ra }}$ satisfies property A from section 2.4, and all the lemmas from Section 3 hold.

As a corollary of Proposition 8.2, we have the following statements which we are going to use later.

Lemma 8.3. Let $\Delta$ be a g-reduced diagram over $\mathcal{R}_{r a}, \partial(\Delta)=p_{1} q_{1} s^{-1} q_{2}^{-1}$, obtained by gluing two diagrams $\Gamma$ and $\Pi$ where

(i) $\partial(\Gamma)=p_{1} q_{1} p_{2}^{-1} q_{2}^{-1}, \phi\left(p_{1}\right)$ is a subword of $R(h, z)$ for some reduced word $h$ over $\mathbb{S}$ and some $z \in \mathbf{K} \cup \mathbf{K}^{-1}, p_{1}$ starts and ends with $\mathbf{R}$-edges; $q_{1}$ and $q_{2}$ do not contain $\mathbf{R}$-edges; $\Gamma$ is a diagram of rank 0 (that is over $Z(\mathbb{S}, a)$ );

(ii) $\Pi$ is a cell of corresponding to a relation $v^{n}=1$ in $\mathcal{R}_{\text {ra }}$ of rank $\geq 1, \partial(\Pi)=p_{2} s^{-1}$;

(iii) $v$ starts with an $\mathbf{R}$-letter $r, \phi\left(p_{2}\right) \equiv v^{l} r$ where $l>1 / 2 \varepsilon n$.

Then there exists a diagram $\Delta^{\prime}$ over $\mathcal{R}_{\text {ra }}$ which has the same boundary label as $\Delta, \partial\left(\Delta^{\prime}\right)=$ $p_{1}^{\prime} q_{1}^{\prime}\left(s^{\prime}\right)^{-1}\left(q_{2}^{\prime}\right)^{-1}$ where $\phi\left(p_{1}^{\prime}\right) \equiv \phi\left(p_{1}\right), \phi\left(q_{1}^{\prime}\right) \equiv \phi\left(q_{1}\right), \phi\left(s^{\prime}\right) \equiv \phi(s), \phi\left(q_{2}^{\prime}\right) \equiv \phi\left(q_{2}\right)$, and

(1) $\Delta^{\prime}$ contains a subdiagram $\Pi^{\prime}$ where $\phi\left(\partial\left(\Pi^{\prime}\right)\right) \equiv u^{n}$ where $u$ is a cyclically $\mathbf{R}$-reduced word,

(2) $\left|\partial\left(\Pi^{\prime}\right) \cap p_{1}^{\prime}\right|>6|u|$,

(3) $\Delta^{\prime} \backslash \Pi^{\prime}$ is of rank 0.

Proof. Consider the $\mathbf{R}$-bands in $\Gamma$ starting on $p_{2}$. Since $v$ is a period, it is cyclically $\mathbf{R}$-reduced (by Proposition 8.2 and the definition of periods from Section 3. Since $q_{1}$ and $q_{2}$ are of $\mathbf{R}$-length 0 , every $\mathbf{R}$-band starting on $p_{2}$ ends on $p_{1}$. Since $\phi\left(p_{1}\right)$ is a subword of an $\mathbf{R}$-reduced word $R(h, z)$, every $\mathbf{R}$-bands starting on $p_{1}$ ends on $p_{2}$. Hence the $\mathbf{R}$-projections of words $\phi\left(p_{1}\right)$ and $\phi\left(p_{2}\right)$ are identical (the labels of $\mathbf{R}$-edges in all cells of a $\mathbf{R}$-band are the same). 
Therefore $\phi\left(p_{1}\right) \equiv u^{l} r$ where $|u|_{\mathbf{R}}=|v|_{\mathbf{R}}, u$ starts with $r$. Since $u$ and $u^{2}$ are subwords of an $\mathbf{R}$-reduced word $R(h, z)$ (since $l \geq \varepsilon n>2$ ), $u$ is cyclically $\mathbf{R}$-reduced. In particular, $u$ is an essential word.

Let $\mathcal{T}$ and $\mathcal{T}^{\prime}$ be the first and the last maximal $\mathbf{R}$-bands in $\Gamma$ starting on $p_{1}$ (we count the R-bands from $\left(p_{1}\right)_{-}$to $\left.\left(p_{1}\right)_{+}\right)$. Consider the subdiagram $\Gamma_{1}$ of $\Gamma$ bounded by the top side of $\mathcal{T}, p_{1}$, $p_{2}$ and the connecting line of $\mathcal{T}^{\prime}$ (so this subdiagram is equal to $\Gamma$ minus $\mathcal{T}^{\prime}$ ). Since all cells in $\mathcal{T}^{\prime}$ are commutativity cells, $x_{1}=\phi\left(\operatorname{top}\left(\mathcal{T}^{\prime}\right)\right)=\phi\left(\operatorname{bot}\left(\mathcal{T}^{\prime}\right)\right)$. The subdiagram of $\Gamma$ bounded by a side of $\mathcal{T}^{\prime}$ and $q_{1}$ does not contain $\mathbf{R}$-bands, so $\phi\left(q_{1}\right)=x_{1}$ modulo $a$-relations. Similarly $\phi\left(q_{2}\right)=x_{2}$ modulo $a$-relations where $x_{2}$ is the word written in a side of $\mathcal{T}$. Clearly $\left|x_{1}\right|_{\mathbf{R}}=\left|x_{2}\right|_{\mathbf{R}}=0$.

Let $\bar{p}_{1}$ be the path $p_{1}$ without the last edge, $\bar{p}_{2}$ be $p_{2}$ without the last edge. Then $\phi\left(\bar{p}_{1}\right) \equiv u^{l}$, $\phi\left(\bar{p}_{2}\right) \equiv v^{l}$,

$$
u^{l} x_{1}=x_{2} v^{l}
$$

modulo $a$-relations and $r a$-relations.

Similarly every subword of the cyclic word $u$ is equal to a subword of the same $\mathbf{R}$-length of the cyclic word $v$ up to multiples of zero length. Hence $v$ is cyclically reduced by Lemma 3.3. Therefore $v$ is essential.

Suppose that $u$ is not simple in rank 0. By Lemma 3.25 there exists a word $w$ which is simple in rank 0 (and so $w$ is simple in rank $1 / 2$ because $\mathcal{R}_{r a}$ does not contain hubs), $|w|_{\mathbf{R}}<|u|_{\mathbf{R}}$, and a word $t \in \mathbf{0}(w)$ such that $u=x w^{b} t x^{-1}$ in rank 0 , for some word $x$ and some integer $b \neq 0$. Since $u$ is cyclically reduced, $|x|_{\mathbf{R}}=0$ and $\left|w^{b}\right|_{\mathbf{R}}=|u|_{\mathbf{R}}$.

Therefore by $u^{l}=x w^{b l} t_{1} x^{-1}$ for some $t_{1} \in \mathbf{0}(w)$ (since $\mathbf{0}(w)$ is normalized by $w$ ). Using (23), we get

$$
u^{l} x_{1}=x w^{b l} t_{1} x^{-1} x_{1}=x_{2} v^{l}
$$

in rank 0 , where both $w$ and $v$ are simple in rank 0 , the $\mathbf{R}$-lengths of $x, x_{1}, t_{1}, x_{2}$ are equal to 0 (for $x, x_{1}, x_{2}$ we have established it above, for $t_{1}$, this follows from the definition of $\mathbf{0}(w)$ since $w$ is cyclically R-reduced). Now we can apply Lemma 3.32 and conclude that $v=y w^{ \pm 1} t_{2} y^{-1}$ in rank 0 for some word $y$ and some $t_{2} \in \mathbf{0}(w)$. This contradicts the fact that $v$ is simple in rank 0 (since $\left.|w|_{\mathbf{R}}<|u|_{\mathbf{R}}=|v|_{\mathbf{R}}\right)$.

Thus $u$ is simple in rank 0 (and so it is simple in rank 1/2). Then again by Lemma 3.32, we can conclude that

$$
x_{2} v^{ \pm 1} t_{2} x_{2}^{-1}=u
$$

in rank 0 for some $t_{2} \in \mathbf{0}(v)$. Suppose that the exponent of $v$ in $(24)-1$, that is $x_{2} v^{-1} t_{2} x_{2}^{-1}=u$. Therefore

$$
u^{l}=x_{2}\left(v^{-1} t_{2}\right)^{l} x_{2}^{-1}=x_{2} v^{-l} t^{\prime} x_{2}^{-1}
$$

for some $t^{\prime} \in \mathbf{0}(v)$ (since $\mathbf{0}(v)$ is normalized by $v$ ). By (23),

$$
x_{2}^{-1} u^{l}=v^{l} x_{1}^{-1}=v^{-l} t^{\prime} x_{2}^{-1}
$$

whence

$$
v^{2 l}=t^{\prime} x_{2}^{-1} x_{1}
$$


Since $t^{\prime}$ is of $\mathbf{R}$-length 0 (this follows from the definition of $\mathbf{0}(v)$ since $v$ is cyclically $\mathbf{R}$-reduced), $x_{1}$ and $x_{2}$ are also of $\mathbf{R}$-length 0 , we have that $v^{2 l}$ is of $\mathbf{R}$-length 0 which contradicts the fact that $v$ is cyclically $\mathbf{R}$-reduced. This contradiction shows that in fact the exponent of $v$ in (24) is 1 .

By Lemma 3.12, $\left(v t_{2}\right)^{n}=v^{n}$ in rank 0. Hence $u^{n}=x_{2} v^{n} x_{2}^{-1}$ in rank 0 .

Recall that $\phi\left(\bar{p}_{1}\right) \equiv u^{l}$. Let $\bar{\Delta}$ be a diagram with contour $\bar{p}_{1}\left(p_{1}^{\prime}\right)^{-1}$ where $\phi\left(p_{1}^{\prime}\right) \equiv u^{l}$, which is a result of gluing two mirror copies $\Pi^{\prime}$ and $\bar{\Pi}$ with boundary labels $u^{ \pm n}$ over $\mathcal{R}_{r a}$ where $p_{1}^{\prime}$ is a part of the boundary of $\Pi^{\prime}, \bar{p}_{1}$ is a part of the boundary of $\Pi$. Consider the union of $\Delta$ and $\bar{\Delta}$. Notice that $\left|p_{1}^{\prime}\right| \geq l|u| \geq \frac{1}{2} \varepsilon n|u|>6|u|$. It remains to observe that in the union of $\Delta$ and $\bar{\Delta}$, the $\bar{\Pi}$ is connected with $\Pi$ by a path with label $x_{2}$, hence this pair of subdiagrams and the connecting path can be replaced by a diagram of rank 0 corresponding to the equality $u^{n}=x_{2} v^{n} x_{2}^{-1}$. The lemma is proved.

\section{$9 \quad$ The group $H_{k r a}$}

Recall that in Section 7 we considered the set of defining relations $Z(\mathbb{S}, a)$ which is the union of the set of all $a$-relations and the set $Z(\mathbb{S})$ of relations corresponding to the $S$-machine $\mathbb{S}$.

Now consider the union $Z(\mathbb{S}, \Lambda, r a)=Z(\mathbb{S}, a) \cup\{\Lambda(0)\} \cup \mathcal{R}_{r a}$ and the group $H_{k r a}$ generated by $\mathbf{K} \cup \mathbf{R} \cup \mathbf{A}$ subject to the relations from $Z(\mathbb{S}, \Lambda, r a)$. Each relation of $Z(\mathbb{S}, \Lambda, r a)$ is either the hub, an $a$-relation, a (commutativity) ra-relation, or a $\kappa$-relation or belongs to $\mathcal{R}_{r a}$. Burnside relations from $\mathcal{R}_{r a}$ will be called Burnside ra-relations. The corresponding cells in diagrams will be called Burnside ra-cells. The set of all relations from $Z(\mathbb{S}, \Lambda, r a)$ except the hub will be denoted by $Z(\mathbb{S}, r a)$.

As in Section 3, we divide the set of generators of $H_{k r a}$ into the set of 0-letters and the set of non-0-letters.

Our main goal is to prove that the presentation $Z(\mathbb{S}, \Lambda, r a)$ satisfies properties (Z1), (Z2), (Z3) if we consider $\mathbf{K}$ as the set of non-zero letters and the $\mathbf{R} \cup \mathbf{A}$ as the set of 0-letters.

But at first we show how to get rid of Burnside $r a$-cells in diagrams over $Z(\mathbb{S}, r a)$.

As in Section 7, we can consider $\mathbf{K}$ - and $\mathbf{R}$-bands in diagrams over $Z(\mathbb{S}, r a)$. The definition of these bands is the same as in Section 7 (Burnside cells do not belong to $\mathbf{R}$-bands). The only difference is that now $\mathbf{R}$-bands can start and end on Burnside ra-cells.

\subsection{Getting rid of Burnside ra-cells}

Consider (temporarily) $\mathbf{R}$ as the set of non-0-letters and all other letters as 0-letters. We can consider R-bands connecting two Burnside ra-cells (a Burnside ra-cell and a part of the boundary of a diagram) as 0-bonds, and define bonds and contiguity subdiagrams of all ranks. (We preserve the grading of the set of Burnside $r a$-relations $\mathcal{R}_{r a}$.)

Suppose that a disc van Kampen diagram $\Delta$ is obtained by gluing together two disc diagrams $\Theta$ and $\Pi$ over $Z(\mathbb{S}, r a)$ along pieces of their boundaries, where

(1) $\Theta$ is a trapezium $T(W, h)$ with contour $p_{1} q_{1} p_{2}^{-1} q_{2}^{-1}\left(q_{1}, q_{2}\right.$ are the bottom and the top bases);

(2) $\phi(\Pi) \equiv u^{n}$ for some cyclically $\mathbf{R}$-reduced word $u$ over $\mathbf{R} \cup \mathbf{A}$, the diagram $\Pi$ does not contain K-edges;

(3) $\Pi$ and $\Theta$ are glued along a subpath $p$ of $p_{2} ;|p| \geq 3|u|$.

Then we shall say that $\Delta$ has the form $\Theta * \Pi$. 
Suppose that a disc van Kampen diagram $\Delta$ is obtained by gluing together two diagrams $\Pi$ and $\Theta$ where

(1) $\Theta$ is a trapezium $T(W, h)$ with contour $p_{1} q_{1} p_{2}^{-1} q_{2}^{-1}\left(q_{1}, q_{2}\right.$ are the bases);

(2) $\phi(\Pi) \equiv u^{n}$ for some cyclically $\mathbf{R}$-reduced word $u$ over $\mathbf{R} \cup \mathbf{A}$ containing $\mathbf{R}$-letters; $\Pi$ does not contain $\mathbf{K}$-edges;

(3) $\Pi$ and $\Theta$ are glued along a subpath $p$ of $p_{1}$.

Then we shall say that $\Delta$ has the form $\Pi \circ \Theta$. It is easy to see that

$$
T(W, h) * \Pi=\Pi \circ T\left(W \cdot h, h^{-1}\right)
$$

(this means that if the right hand side of the equality exists then both sides exist and are equal).

Lemma 9.1. For every van Kampen disc diagram $\Delta$ of the form $\Theta * \Pi$ there exists a van Kampen disc diagram $\Delta^{\prime}$ of the form $\Pi^{\prime} \circ \Theta^{\prime}$ with the same boundary label and such that:

(i) the label of the bottom (top) base of $\Theta$ is equal to the label of the bottom (top) base of $\Theta^{\prime}$;

(ii) The histories of the trapezia $\Theta$ and $\Theta^{\prime}$ are equal modulo Burnside relations (see Figure 15).

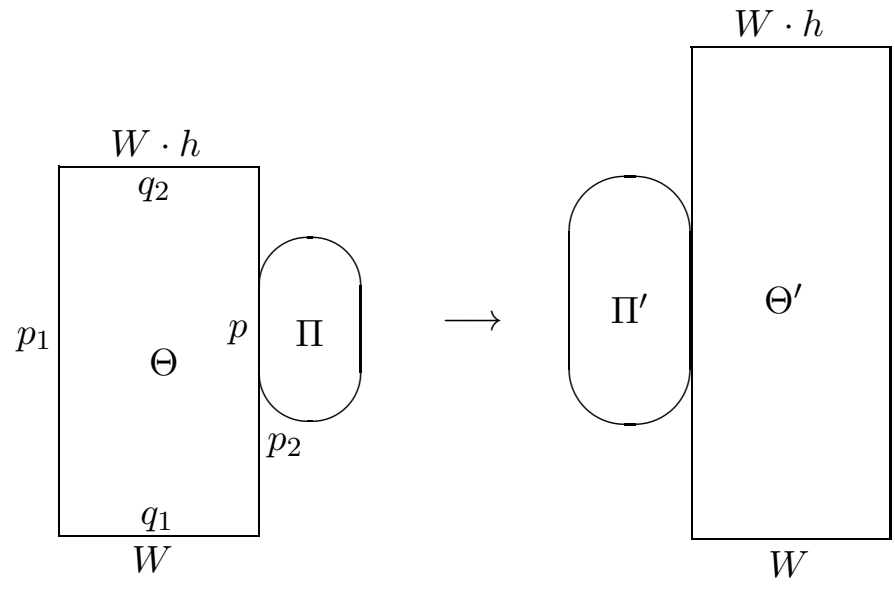

Fig. 15.

Proof. Let $\Theta=T(W, h)$. Then $\phi\left(p_{2}\right) \equiv R(h, z)^{-1}$ where $z \in \mathbf{K} \cup \mathbf{K}^{-1}$ is the last letter of $W$. Let $p$ be the common subpath of the contour of $\Pi$ and the right side of the trapezium $\Theta, \phi(\partial(\Pi)) \equiv u^{n}$ for a cyclically $\mathbf{R}$-reduced word $u$. Then $\phi(p) \equiv R(h, z)^{-1}$ contains a cube of a cyclic shift of $u$ as a subword. Without loss of generality we can assume that this cyclic shift is $u$ itself. The word $R(h, z)^{-1}$ is a product of blocks of the form $R(\tau, z), \tau \in \mathbb{S}$, each of which is either a one-letter word $r \in \mathbf{R} \cup \mathbf{R}^{-1}$ or a two-letter word $(r a)^{ \pm 1}$ where $r \in \mathbf{R}, a \in \mathbf{A} \cup \mathbf{A}^{-1}$. Every block is completely determined by its $\mathbf{R}$-letter. Consider the occurrence of $u^{3}$ in $R(h, z)^{-1}$.

The word $u^{3}$ contains subword $v^{2}$ where $v$ is a cyclic shift of $u$ and the first occurrence of $v$ (in $v^{2}$ ) starts with the beginning of a block. Suppose that $v$ does not end with the end of a block. Then the second occurrence of $v$ in $v^{2}$ starts in the middle of a block, so the first letter of $v$ is both a beginning of a block and an end of a block. Therefore this letter belongs to $\mathbf{A}^{ \pm 1}$. Hence $v \equiv a r_{1} v^{\prime} r_{2}$ where $a \in \mathbf{A} \cup \mathbf{A}^{-1}, r_{1} \in \mathbf{R}^{-1}, r_{2} \in \mathbf{R}, a r_{1}$ and $r_{2} a$ are blocks. But then the second letter of the second occurrence of $v$ must be $r_{1}$, and it must be the beginning of a block. Thus the same $\mathbf{R}$-letter $r_{1}$ is a beginning and the end of a block. This contradicts the fact that every block is completely determined by its $\mathbf{R}$-letter. 
Hence $v$ starts with a beginning of a block and ends with the end of a block. Therefore $v \equiv R\left(h_{1}, z\right)$ for some cyclically reduced word $h_{1}$ over $\mathbb{S}$. Thus $h_{1}^{2}$ is a subword of $h$. Let $h \equiv h^{\prime} h_{1}^{2} h^{\prime \prime}$ for some words $h^{\prime}, h^{\prime \prime}$. Then $W \cdot h^{\prime}$ is in the domain of $h_{1}^{2}$. By Lemma 6.5(iv), then $W \cdot h^{\prime}$ is in the domain of $h_{1}^{s}$ for every integer $s$. In particular, $W \cdot h^{\prime}$ is in the domain of $h_{1}^{2-n}$. By Lemma 6.5(ii),

$$
W \cdot h=W \cdot h^{\prime} h_{1}^{2} h^{\prime}=W \cdot h^{\prime} h_{1}^{2-n} h^{\prime \prime} .
$$

Since $v \equiv R\left(h_{1}, z\right)$ is a cyclic shift of $u$, one can read $v^{n}$ on the boundary of $\Pi$, and the boundary label of $\Theta * \Pi$ is freely equal as a cyclic word to

$$
\begin{aligned}
& W R\left(h^{\prime \prime}, z\right)^{-1} v^{n-2} R\left(h^{\prime}, z\right)^{-1}(W \cdot h)^{-1} L\left(h, z^{\prime}\right)= \\
& W R\left(h^{\prime \prime}, z\right)^{-1} R\left(h_{1}^{2-n}, z\right)^{-1} R\left(h^{\prime}, z\right)^{-1}(W \cdot h)^{-1} L\left(h, z^{\prime}\right)= \\
& W R\left(h^{\prime} h_{1}^{2-n} h^{\prime \prime}, z\right)^{-1}(W \cdot h)^{-1} L\left(h^{\prime} h_{1}^{2} h^{\prime \prime}, z^{\prime}\right)
\end{aligned}
$$

where $z^{\prime}$ is the first letter of $W$.

Consider now the trapezium $\Theta^{\prime}=T\left(W, h^{\prime} h_{1}^{2-n} h^{\prime \prime}\right)$ which exists by Lemma $7.5(\mathrm{i})$ since $W$ is in the domain of $h^{\prime} h_{1}^{2-n} h^{\prime \prime}$. Since $W \cdot h^{\prime} h_{1}^{2-n} h^{\prime \prime}=W \cdot h$, we can assume that the top base label of $\Theta^{\prime}$ is the same as the top base label of $\Theta$, that is $W \cdot h$.

The boundary label of $\Theta^{\prime}$ is thus freely equal (as a cyclic word) to

$$
W R\left(h^{\prime} h_{1}^{2-n} h^{\prime \prime}, z\right)^{-1}(W \cdot h)^{-1} L\left(h^{\prime} h_{1}^{2-n} h^{\prime \prime}, z^{\prime}\right) .
$$

Notice that by Proposition 8.2 there exists a van Kampen disc diagram $\Pi^{\prime}$ containing only $a$-cells, $r a$-cells and Burnside ra-cells, that has boundary label $L\left(h_{1}, z^{\prime}\right)^{n}$. We can glue $\Pi^{\prime}$ and $\Theta^{\prime}$ along the path labeled by $L\left(h_{1}, z^{\prime}\right)^{2-n}$, we get a diagram $\Delta^{\prime}$ of the form $\Pi^{\prime} \circ \Theta^{\prime}$. The boundary label of this diagram will be freely equal to

$$
W R\left(h^{\prime} h_{1}^{2-n} h^{\prime \prime}, z\right)^{-1}(W \cdot h)^{-1} L\left(h^{\prime} h_{1}^{2} h^{\prime \prime}, z^{\prime}\right) .
$$

Comparing this with (25) we conclude that the boundaries of $\Delta$ and $\Delta^{\prime}$ are freely equal. The labels of the bottom (top) bases of $\Theta$ and $\Theta^{\prime}$ are equal by construction, the history $h \equiv h^{\prime} h_{1}^{2} h^{\prime \prime}$ of $\Theta$ is equal to the history $h^{\prime} h^{2-n} h^{\prime \prime}$ of $\Theta^{\prime}$ modulo Burnside relations, as required.

Lemma 9.1 allows us to move subdiagrams with boundary labels of the form $u^{n}$ from one side of a trapezium to another side, preserving the history of the trapezium modulo Burnside relations. The next lemma is the first application of this trick.

Lemma 9.2. For every disc diagram $\Delta$ over $Z(\mathbb{S}, r a)$ there exists another disc diagram $\Delta^{\prime}$ with the same boundary label and without $\mathbf{K}$-annuli.

Proof. We can assume that $\Delta$ contains exactly one $\mathbf{K}$-annulus $\mathcal{B}$ and $\partial(\Delta)$ is equal to $t=\operatorname{top}(\mathcal{B})$. Then the subdiagram $\Delta^{\prime}$ bounded by $\operatorname{bot}(\mathcal{B})$ is a diagram over $\mathcal{R}_{r a}$. We can assume that this subdiagram is $g$-reduced, and that the $\mathbf{K}$-annulus $\mathcal{B}$ is reduced.

By Proposition 8.2 $\Delta^{\prime}$ is an $A$-map. Suppose that $\Delta^{\prime}$ contains a Burnside $r a$-cell. By Lemma 2.6 there exists a Burnside $r a$-cell $\Pi$ in $\Delta^{\prime}$ and its contiguity subdiagram $\Gamma$ to $\operatorname{bot}(\mathcal{B})$ with contiguity degree at least $\varepsilon$. By Lemma 8.3, we can replace $\Pi \cup \Gamma$ by a new subdiagram $\Psi$ with the same boundary label which contains a subdiagram $\Pi^{\prime}$ such that $\phi\left(\partial\left(\Pi^{\prime}\right)\right) \equiv u^{n}$ for some word $u$ and $\left|\partial\left(\Pi^{\prime}\right) \cap \operatorname{bot}(\mathcal{B})\right|>6|u|, \Psi \backslash \Pi^{\prime}$ is a diagram of rank 0. Now using Lemma 9.1, we can move $\Pi^{\prime}$ through $\mathcal{B}$ without producing new $\mathbf{K}$-annuli. 
After a number of such transformations we replace $\Delta$ by a new disc diagram $\bar{\Delta}$ with the same boundary label containing exactly one $\mathbf{K}$-annulus which bounds a subdiagram without Burnside ra-cells. By Lemma 7.3, part 1, applied to the subdiagram bounded by that $\mathbf{K}$-band, we can replace that subdiagram by a subdiagram without $\mathbf{K}$-edges. Since this operation reduces the number of $\mathbf{K}$-annuli, the statement of the lemma follows.

Let $\Delta$ be an annular diagram over $Z(\mathbb{S}, r a)$ with inner contour $p_{1}$ and outer contour $p_{2}$, and let $q$ be a path in $\Delta$. Suppose that $\Delta, q$ satisfy the following conditions:

(T1) $p_{1}$ and $p_{2}$ are sides of $\mathbf{K}$-annuli $\mathcal{T}_{1}$ and $\mathcal{T}_{2}$ respectively; $\phi\left(p_{1}\right) \neq 1$ modulo $Z(\mathbb{S}, r a)$;

(T2) $q$ connects $\left(p_{1}\right)_{-}=\left(p_{1}\right)_{+}$with $\left(p_{2}\right)_{-}=\left(p_{2}\right)_{+}$; the first and the last edges of $q, e_{1}, e_{2}$, are K-edges;

(T3) Every maximal $\mathbf{K}$-band in $\Delta$ is an annulus surrounding the hole of $\Delta$.

Maximal K-bands in $\Delta$ divide $\Delta$ into annular subdiagrams (without $\kappa$-cells) which will be called chambers. We count the chambers of $\Delta$ from $p_{1}$ to $p_{2}$.

Lemma 9.3. Suppose that all chambers of $\Delta$ except the last one, $\bar{\Delta}$, do not contain Burnside ra-cells and $\phi(q)$ is $\mathbf{K}$-reduced.

Then there exists another annular diagram $\Delta^{\prime}$ over $Z(\mathbb{S}, a)$ (that is without Burnside ra-cells) with contours $p_{1}^{\prime}$ and $p_{2}^{\prime}$, and a path $q^{\prime}$ in $\Delta^{\prime}$ satisfying the conditions (T1),(T2), (T3) such that $\phi\left(p_{1}^{\prime}\right)=\phi\left(p_{1}\right), \phi\left(p_{2}^{\prime}\right)=\phi\left(p_{2}\right)$ modulo Burnside relations, $\phi\left(q^{\prime}\right)=\phi(q)$ modulo $Z(\mathbb{S}, r a)$, $\phi\left(q^{\prime}\right)$ is $\mathbf{K}$-reduced.

Proof. Among all pairs $(\Delta, q)$ satisfying the conditions of the lemma and having the same boundary labels of contours $p_{1}, p_{2}$ modulo Burnside relations and the same labels of $q$ modulo $Z(\mathbb{S}, r a)$ let us choose a pair where $\Delta$ has the smallest possible number of chambers and the last chamber $\bar{\Delta}$ has the smallest type in the sense of Section 3.

First of all let us reduce all chambers of $\Delta$. We can get rid of $j$-pairs of Burnside ra-cells by first replacing the path $q$ by a path avoiding the $j$-pair and its connecting path and then reducing the $j$-pair. Similarly, if $\Delta$ contains a disc subdiagram $\Psi$ over $Z(\mathbb{S}, a)$ containing $r$-cells, whose boundary label is a word over $\mathbf{A}$ and is equal to 1 modulo $a$-relations, then we can replace this subdiagram by a subdiagram with the same boundary label containing no $r$-cells. The resulting diagram will satisfy conditions (T1), (T2), (T3). These operations will not increase the number of chambers in $\Delta$ or the type of $\bar{\Delta}$ or the K-length of $\phi(q)$. Thus we can assume that $\Delta$ does not contain disc subdiagrams whose boundary labels are words over $\mathbf{A}$ equal 1 modulo $a$-relations. In particular, all chambers except for the last one are $Z(\mathbb{S}, a)$-reduced in the sense of Section 7.2.

We can also reduce all $\mathbf{K}$-annuli in $\Delta$ and also the bands $\mathcal{T}_{1}$ and $\mathcal{T}_{2}$ without changing the $\mathbf{K}$ length of $q$. Notice that we do not assume that $\mathcal{T}_{1}$ and $\mathcal{T}_{2}$ are cyclically reduced because it may be impossible to reduce the annuli $\mathcal{T}_{1}$ and $\mathcal{T}_{2}$ without changing $q$.

Hence we can assume that $\bar{\Delta}$ is g-reduced in the sense of Section 3 as a diagram over $Z(\mathbb{S}, r a)$ and $\Delta \backslash\left(\bar{\Delta} \cup \mathcal{T}_{2} \cup \mathcal{T}_{1}\right)$ which is a diagram over $Z(\mathbb{S}, a)$ is $Z(\mathbb{S}, a)$-reduced.

Since $\phi(q)$ is $\mathbf{K}$-reduced in $H_{k r a}, q$ intersects every $\mathbf{K}$-annulus in $\Delta$ only once. Therefore the $\mathbf{K}$-length of $q$ is equal to the number of $\mathbf{K}$-annuli in $\Delta$.

Let $\mathcal{B}$ be a maximal R-band connecting the contours of $\Delta^{\prime \prime}=\Delta \backslash\left(\bar{\Delta} \cup \mathcal{T}_{2}\right)$. Such a band exists because if every $\mathbf{R}$-band starting on the boundary of $\mathcal{T}_{1}$ ends on the boundary of $\mathcal{T}_{1}$ then the label of a side of $\mathcal{T}_{1}$ is equal to 1 modulo $Z(\mathbb{S}, r a)$, a contradiction with (T1). 
Since the label of a side of $\mathcal{B}$ is equal to the label of the portion of $q$ in that subdiagram multiplied by words of $\mathbf{K}$-length 0 (read along the boundaries of the subdiagram), the label of any side of $\mathcal{B}$ is $\mathbf{K}$-reduced.

By Proposition 8.2, chamber $\bar{\Delta}$ is an $A$-map. Let $o=\left(e_{2}\right)_{-}$. Let $s$ be the inner contour of $\bar{\Delta}$, and let $t$ be the outer contour of $\bar{\Delta}$. We consider $o$ as the beginning and the end of $t$.

Suppose that $\bar{\Delta}$ contains Burnside $r a$-cells. Then by Lemma 2.5, part c), there exists a Burnside $r a$-cell $\Pi$ in $\bar{\Delta}$ and its contiguity subdiagram $\Gamma$ of rank 0 to one of the contours $s$ or $t$ of contiguity degree $\geq \varepsilon$.

Replacing $\Gamma$ (if necessary) by a smaller contiguity diagram, we can assume that conditions (i)-(iv) of Lemma 8.3 hold. By this lemma, we can replace $\Gamma \cup \Pi$ by a subdiagram $\Psi$ with the same boundary label, which has a subdiagram $\Pi^{\prime}$ such that $\partial\left(\Pi^{\prime}\right) \equiv u^{n}$ where $u$ is a cyclically R-reduced word and $\left|\partial\left(\Pi^{\prime}\right) \cap s\right|>6|u|$ or $\left|\partial\left(\Pi^{\prime}\right) \cap t\right|>6|u|$ and $\Psi \backslash \Pi^{\prime}$ has rank 0 .

Suppose first that $\left|\partial\left(\Pi^{\prime}\right) \cap t\right|>6|u|$. By Lemma 9.1 we can move $\Pi^{\prime}$ through $\mathcal{T}_{2}$ replacing $\mathcal{T}_{2}$ by a $\mathbf{K}$-band with a history which is equal to the history of $\mathcal{T}_{2}$ modulo Burnside relations. The path $q$ can be replaced in the new annular diagram by a path with the same label modulo $Z(\mathbb{S}, r a)$. This operation decreases the type of the last chamber of $\Delta$.

Suppose now that $\left|\partial\left(\Pi^{\prime}\right) \cap s\right|>6|u|$. Let $x=\partial\left(\Pi^{\prime}\right) \cap s$. Consider the annular subdiagram $\Delta^{\prime \prime}$ bounded by $s$ and $p_{1}$. The diagram $\Delta^{\prime \prime}$ is a $Z(\mathbb{S}, a)$-reduced diagram. By Lemma 7.3 every maximal $\mathbf{R}$-band starting on $x$ must end on $p_{1}$. Indeed otherwise the $\mathbf{R}$-band would have two intersections with a reduced $\mathbf{K}$-band in a disc subdiagram of $\Delta^{\prime \prime}$ which contradicts Lemma 7.3, part 2. Since such an $\mathbf{R}$-band cannot intersect twice a $\mathbf{K}$-annulus, and $\phi(q)$ is a $\mathbf{K}$-reduced word, the label of a side of any of these $\mathbf{R}$-bands is a $\mathbf{K}$-reduced word. There exists a disc subdiagram $\Theta$ of $\Delta^{\prime \prime}$ containing at least half of these $\mathbf{R}$-bands, bounded by a subpath $y$ of $x$, a subpath of $p_{1}$ and by two $r$-bands. Now $\Theta$ is a trapezium because it satisfies conditions (TR1) and (TR2). By Lemma 7.5, $\Theta$ is a normal trapezium. The union of $\Theta$ and $\Pi^{\prime}$ is, by definition, equal to $\Theta * \Pi^{\prime}$. As before we can move $\Pi^{\prime}$ through $\Theta$ replacing $\Theta$ by a trapezium with the same history modulo Burnside relations. Again we decrease the type of $\Delta$.

The new diagram satisfies conditions (T1), (T2), (T3) and has a smaller type than $\Delta$.

Thus we can complete the proof by induction on the type of $\Delta$.

Lemma 9.3 implies the following stronger statement.

Lemma 9.4. The conclusion of Lemma 9.3 holds if one removes the restriction that only the last chamber contains Burnside ra-cells.

Proof. Since $\phi(q)$ is reduced, we can assume that it crosses the connecting line of every K-annulus only once.

Let us use induction on the number of chambers in $\Delta$. If the number of chambers is 1 , we can apply Lemma 9.3. Consider the diagram $\Delta_{1}=\Delta \backslash\left(\bar{\Delta} \cup \mathcal{T}_{2}\right)$ where $\bar{\Delta}$ is the last chamber. Let $q_{1}$ be the part of $q$ in $\Delta_{1}$. The number of chambers in $\Delta_{1}$ is smaller than the number of chambers in $\Delta$. Therefore we can apply the induction hypothesis and conclude that there exists a diagram $\Delta_{2}$ and a path $q_{2}$ which satisfy the conclusion of Lemma 9.3.

Since the label of the outer contour of $\Delta_{2}$ is equal to the label of the outer contour of $\Delta_{1}$ modulo Burnside relations, we can glue to the outer contour of $\Delta_{2}$ a diagram over $\mathcal{R}_{r a}$ such that the resulting diagram $\Delta_{3}$ has the same outer boundary label as $\Delta_{1}$. 
Now replace the subdiagram $\Delta_{1}$ in $\Delta$ by $\Delta_{3}$ and the subpath $q_{1}$ of $q$ by $q_{2}$. Let $\left(\Delta_{4}, q_{4}\right)$ be the resulting pair of an annular diagram and a cutting path. Reducing if necessary, the last $\mathbf{K}$-annulus of subdiagram $\Delta_{3}$ of $\Delta_{4}$, we obtain a pair $\left(\Delta_{5}, q_{5}\right)$ satisfying conditions of Lemma 9.3. Applying that lemma, we complete the proof.

The following lemma is an algebraic consequence of Lemma 9.4.

Lemma 9.5. Let $P_{1}, P_{2}$ be words over $\mathbf{A} \cup \mathbf{R}$ which are not equal to 1 modulo $Z(\mathbb{S}, r a)$. Let $A^{-1} P_{1} A=P_{2}$ in $H_{k r a}$ for some word $A$ which is $\mathbf{K}$-reduced in $H_{k r a}$ and starts and ends with a letter $z \in \mathbf{K} \cup \mathbf{K}^{-1}$. Then $A=R_{1} B R_{2}$ in $H_{k r a}$ where $R_{1}=L\left(h_{1}, z\right), R_{2}=R\left(h_{2}, z\right)$, $z$ is in the domain of $h_{1}, h_{2}^{-1}, B$ is a reduced admissible word starting and ending with $z_{1}$ such that $R_{1} z_{1}=z R\left(h_{1}, z\right), z_{1} R_{2}=L\left(h_{2}, z_{1}\right) z$ modulo $Z(\mathbb{S}, a)$.

Proof. Equality $P_{2}=A^{-1} P_{1} A$ gives us an annular diagram $\Delta$ with inner contour $p_{1}$ labeled by $P_{1}$, outer contour $p_{2}$ labeled by $P_{2}$ and a cutting path $q$ labeled by $A$.

The connecting line of any $\mathbf{K}$-annulus in $\Delta$ not surrounding the hole of $\Delta$ cannot cross $q$ because otherwise by Lemma 9.2 we would be able to decrease the $\mathbf{K}$-length of $q$ by going along the boundary of such a $\mathbf{K}$-annulus instead of crossing it. Hence by Lemma 9.2 we can assume that $\Delta$ does not contain $\mathbf{K}$-annuli not surrounding the hole of $\Delta$.

Let $e$ and $f$ be the first and the last edges of $q$. Since $e$ and $f$ are $\mathbf{K}$-edges, they belong to the first and the last $\mathbf{K}$-annulus of $\Delta$ counting from $p_{1}$ to $p_{2}$. The label of $p_{1}$ is equal modulo $Z(\mathbb{S}, r a)$ to the label of a side $p_{1}^{\prime}$ of the first $\mathbf{K}$-annulus in $\Delta$ and the label of $p_{2}$ is equal in $Z(\mathbb{S}, r a)$ to the label of a side of the last $\mathbf{K}$-annulus in $\Delta$. Hence we can assume that $p_{1}$ and $p_{2}$ are the sides of these annuli.

Thus $\Delta$ satisfies conditions (T1), (T2), (T3). Since $\phi(q)$ is $\mathbf{K}$-reduced, we can apply Lemma 9.4 and conclude that there exists another annular diagram $\Delta^{\prime}$ over $Z(\mathbb{S}, a)$ with contours $p_{1}^{\prime}$ and $p_{2}^{\prime}$, and a path $q^{\prime}$ in $\Delta^{\prime}$ satisfying the conditions (T1),(T2), (T3) such that $\phi\left(p_{1}^{\prime}\right)=\phi\left(p_{1}\right), \phi\left(p_{2}^{\prime}\right)=\phi\left(p_{2}\right)$ modulo Burnside relations, $\phi\left(q^{\prime}\right)=\phi(q)=A$ modulo $Z(\mathbb{S}, r a), \phi\left(q^{\prime}\right)$ is K-reduced.

Assume that every maximal $\mathbf{R}$-band starting on the first $\mathbf{K}$-annulus of $\Delta^{\prime}$ does not end on $p_{2}^{\prime}$. Then the label of $p_{1}^{\prime}$ is equal to 1 modulo $Z(\mathbb{S}, r a)$, a contradiction. Hence $\Delta^{\prime}$ contains an $\mathbf{R}$-band $\mathcal{B}$, starting on $p_{1}^{\prime}$ and ending on $p_{2}^{\prime}$.

Let $t$ be a side of $\mathcal{B}$. Then $q$ is homotopic to $\left(p_{1}^{\prime}\right)^{s}\left(p_{1}^{\prime \prime}\right) t p_{2}^{\prime \prime}$ for some integer $s$, where $p_{1}^{\prime \prime}$ is a subpath of $p_{1}^{\prime}$ and $p_{2}^{\prime \prime}$ is a subpath of $p_{2}^{\prime}$. By Lemma $7.2 \phi(t) \equiv B$ is an admissible word starting and ending with a $\mathbf{K}$-letter $z^{\prime}$. The $\mathbf{K}$-length of $B$ is the same as the $\mathbf{K}$-length of $A$ (since $\mathcal{B}$ intersects each of the $\mathbf{K}$-annuli exactly once), whence $B$ is reduced in $H_{k r a}$.

The word $\phi\left(\left(p_{1}^{\prime}\right)^{s}\left(p_{1}^{\prime \prime}\right)\right.$ is written on the top side of $z^{\prime}$-band, so it is equal to $R_{1} \equiv L\left(h_{1}, z\right)$ for some $h_{1}$. Similarly $\phi\left(p_{2}^{\prime \prime}\right) \equiv R\left(h_{2}, z\right)$ for some $h_{2}$. The last equalities from the lemma follow from Lemma 7.1.

Lemma 9.6. Let $A$ be a $\mathbf{K}$-reduced word without $\mathbf{R}$-letters, starting and ending with letters $z_{1}, z_{2}$ from $\mathbf{K} \cup \mathbf{K}^{-1}$, and suppose that for some words $P_{1}$ and $P_{2}$ of $\mathbf{K}$-length $0, P_{1} \neq 1$ modulo $Z(\mathbb{S}$, ra), we have $A^{-1} P_{1} A=P_{2}$ modulo $Z(\mathbb{S}, r a)$. Then:

(1) $P_{1}=L\left(h, z_{1}\right), P_{2}=R\left(h, z_{2}\right)$ modulo $Z(\mathbb{S}, r a)$ for some word $h$ over $\mathbb{S}$;

(2) $A$ is a reduced admissible word;

(3) $A \cdot h=A$. 
Proof. Consider a van Kampen disc diagram $\Delta$ over $Z(\mathbb{S}, r a)$ for the equality $A^{-1} P_{1} A=P_{2}$, $\partial(\Delta)=p_{1} q_{1} p_{2}^{-1} q_{2}^{-1}$ where $\phi\left(p_{i}\right) \equiv P_{i}^{-1}, \phi\left(q_{i}\right) \equiv A, i=1,2$. By Lemma 9.2 we can assume that $\Delta$ does not contain $\mathbf{K}$-annuli. We can also assume that all $\mathbf{K}$-bands in $\Delta$ are reduced. Hence every maximal $\mathbf{K}$-band in $\Delta$ connects $q_{1}$ and $q_{2}$. These maximal $\mathbf{K}$-bands divide $\Delta$ into diagrams over $Z(\mathbb{S}, r a)$ without $\mathbf{K}$-edges, which we shall call chambers as in the annular case before. As in the proof of Lemma 9.4, one can use induction on the number of chambers, Proposition 8.2, Lemma 8.3 and Lemma 9.1, to move all Burnside ra-cells out of $\Delta$. Since only two sides of $\Delta$, namely $p_{1}$ and $p_{2}$ may contain $\mathbf{R}$-edges, only these two sides will change during this process. Their labels will stay the same modulo $Z(\mathbb{S}, r a)$. Hence there exists a van Kampen diagram $\Delta_{1}$ over $Z(\mathbb{S}, a)$ (i.e. over $Z(\mathbb{S}, r a)$ but without Burnside $r a$-cells) with contour $p_{1}^{\prime} q_{1}\left(p_{2}^{\prime}\right)^{-1} q_{2}^{-1}$ where $\phi\left(p_{i}^{\prime}\right)=\phi\left(p_{i}\right)$, modulo Burnside relations $i=1,2$.

Let $\mathcal{T}_{1}$ and $\mathcal{T}_{2}$ be the first and the last $\mathbf{K}$-bands in $\Delta_{1}$ starting on $q_{1}$ and ending on $q_{2}$ (we count the $\mathbf{K}$-bands starting at $p_{1}^{\prime}$ ). Consider the subdiagram $\Delta_{2}$ of $\Delta_{1}$ bounded by $\mathcal{T}_{1}, \mathcal{T}_{2}, q_{1}, q_{2}$ (and containing $\left.\mathcal{T}_{1}, \mathcal{T}_{2}\right)$. Then $\partial\left(\Delta_{2}\right)=p_{1}^{\prime \prime} q_{1}\left(p_{2}^{\prime \prime}\right)^{-1} q_{2}^{-1}$ where $p_{1}^{\prime \prime}$ is a side of $\mathcal{T}_{1}, p_{2}^{\prime \prime}$ is a side of $\mathcal{T}_{2}$.

Considering the subdiagrams of $\Delta_{1}$ bounded by $p_{1}^{\prime}\left(p_{1}^{\prime \prime}\right)^{-1}$ and $p_{2}^{\prime}\left(p_{2}^{\prime \prime}\right)^{-1}$, we conclude that $\phi\left(p_{1}^{\prime}\right)=$ $\phi\left(p_{1}^{\prime \prime}\right), \phi\left(p_{2}^{\prime}\right)=\phi\left(p_{2}^{\prime \prime}\right)$ modulo $Z(\mathbb{S}, r a)$. This gives part (1) of the lemma.

The subdiagram $\Delta_{2}$ is a trapezium (by definition). Hence it is a normal trapezium $T(A, h)$ for some word $h$ (by Lemma 7.5). In particular, $A$ is an admissible word which proves part (2) of the lemma. Moreover, $\phi\left(p_{1}\right)=\phi\left(p_{1}^{\prime}\right)=\phi\left(p_{1}^{\prime \prime}\right)=L\left(h, z_{1}\right)^{-1}, \phi\left(p_{2}\right)=\phi\left(p_{2}^{\prime}\right)=\phi\left(p_{2}^{\prime \prime}\right)=R\left(h, z_{2}\right)^{-1}$ modulo $Z(\mathbb{S}, r a)$ for some word $h$ over $\mathbb{S}$. Since the top base of $\Delta_{2}$ has label $A$, we have $A \cdot h=A$. This gives part (3) of the lemma.

Lemma 9.7. Let $B$ be a $\mathbf{K}$-reduced word, starting with a letter $z \in \mathbf{K} \cup \mathbf{K}^{-1}$ and ending with a letter $z^{\prime} \in \mathbf{K} \cup \mathbf{K}^{-1}$ which does not contain $\mathbf{R}$-letters. Let $U$ be a word over $\mathbf{A}$, let $P, R$ be words of $\mathbf{K}$-length 0 such that $B U R B U z$ is $\mathbf{K}$-reduced, $P \neq 1$ in $Z(\mathbb{S}, r a)$. Suppose that $(B U R B U z)^{-1} P(B U R B U z)$ is a 0 -word $Q$ modulo $Z(\mathbb{S}, r a)$. Then $B U z$ is an admissible word, and there exist 0 -words $P^{\prime}, R^{\prime}$ and $Q^{\prime}$ which are equal modulo $Z(\mathbb{S}$, ra $)$ respectively to $P, R, Q$, such that $\left(B U R^{\prime} B U z\right)^{-1} P^{\prime}\left(B U R^{\prime} B U z\right)=Q^{\prime}$ modulo $Z(\mathbb{S}, a)$.

Proof. Consider a disc diagram $\Delta$ over $Z(\mathbb{S}, r a)$ corresponding to the equality

$$
(B U R B U z)^{-1} P(B U R B U z)=Q,
$$

$\partial(\Delta)=p_{1} q_{1} p_{2}^{-1} q_{2}^{-1}$ where $\phi\left(p_{1}\right) \equiv P, \phi\left(p_{2}\right) \equiv Q, \phi\left(q_{1}\right) \equiv \phi\left(q_{2}\right) \equiv B U R B U z$. Let $q_{1}=s_{1} x_{1} t_{1} s_{1}^{\prime}$, $q_{2}=s_{2} x_{2} t_{2} s_{2}^{\prime}$ where $\phi\left(s_{i}\right) \equiv B, \phi\left(x_{i}\right) \equiv U, \phi\left(t_{i}\right) \equiv R, \phi\left(s_{i}^{\prime}\right) \equiv B U z, i=1,2$. Let $\mathcal{T}_{1}, \mathcal{T}_{2}$ be the first and the last $\mathbf{K}$-bands starting on $s_{1}, \mathcal{T}_{3}, \mathcal{T}_{4}$ be the first and the last $\mathbf{K}$-bands starting on $s_{1}^{\prime}$. Since $B U R$ is cyclically reduced, $\mathcal{T}_{1}, \mathcal{T}_{2}$ end on the first and the last edges of $s_{2}, \mathcal{T}_{3}, \mathcal{T}_{4}$ end on the first and the last edges of $s_{2}^{\prime}$. Then we can assume that $p_{1}$ is a side of $\mathcal{T}_{1}, p_{2}$ is a side of $\mathcal{T}_{4}$. Let $\bar{p}_{1}$ be the side of $\mathcal{T}_{2}$ which connects $\left(s_{2}\right)_{+}$with $\left(s_{1}\right)_{+}$, let $\bar{p}_{2}$ be a side of $\mathcal{T}_{3}$ which connects $\left(s_{2}^{\prime}\right)_{-}$with $\left(s_{1}^{\prime}\right)_{-}$.

By Lemma $9.6 \phi\left(p_{1}\right)=L\left(h_{1}, z\right), \phi\left(p_{2}\right)=R\left(h_{2}, z\right)$ modulo $Z(\mathbb{S}, r a), B$ and $B U z$ are admissible words, and $B \cdot h_{1}=B, B U z \cdot h_{2}=B U z$. Thus as in Lemma 9.4 we can move all Burnside cells out of the subdiagrams $\Delta_{1}$ and $\Delta_{2}$ of $\Delta$ bounded by $p_{1} s_{1}\left(\bar{p}_{1}\right)^{-1} s_{2}^{-1}$ and by $\bar{p}_{2} s_{1}^{\prime} p_{2}^{-1}\left(s_{2}^{\prime}\right)^{-1}$, and replace them by trapezia $T\left(B, h_{1}\right)$ and $T\left(B, h_{2}\right)$ in the expense of possible increasing the number of cells in the subdiagram $\Gamma$ bounded by $\bar{p}_{1} x_{1} t_{1} \bar{p}_{2}^{-1}\left(x_{2} t_{2}\right)^{-1}$. Hence we can assume that $\Delta_{1}=T\left(B, h_{1}\right)$, $\Delta_{2}=T\left(B, h_{2}\right)$. 
Thus all Burnside $r a$-cells in $\Delta$ are in $\Gamma$. Consider the diagram $\Gamma$. By Lemma 9.2 we can assume that $\Gamma$ does not have $\mathbf{K}$-annuli. Since the boundary of $\Gamma$ does not contain $\mathbf{K}$-edges, the entire diagram $\Gamma$ does not have $\mathbf{K}$-edges.

Since $\phi\left(t_{1}\right) \equiv \phi\left(t_{2}\right)$, we can identify $t_{1}$ and $t_{2}$ to obtain an annular diagram $\bar{\Gamma}$ and a path $t$ connecting the contours $x_{2}^{-1} \bar{p}_{1} x_{1}$ and $\bar{p}_{2}$ of $\Gamma$. We can eliminate all $j$-pairs of $\bar{\Gamma}$, replacing $t$ by a path with the same label modulo $Z(\mathbb{S}, r a)$. Hence we can assume that $\bar{\Gamma}$ does not have $j$-pairs. Hence $\bar{\Gamma}$ is an $A$-map by Proposition 8.2.

If $\bar{\Gamma}$ does not have Burnside ra-cells, we are done, because we can again cut $\bar{\Gamma}$ along the path t. Suppose that $\bar{\Gamma}$ contains Burnside ra-cells. Consider $\bar{\Gamma}$ as a map satisfying condition A from Section 2.4 with boundary divided into four parts: $x_{1}, x_{2}, \bar{p}_{1}, \bar{p}_{2}$. Recall that paths $x_{1}, x_{2}$ do not contain R-edges. By Lemma 2.5, there exists a Burnside $r a$-cell $\Pi$ and a contiguity subdiagram $\Gamma^{\prime}$ of rank 0 of $\Pi$ to $\bar{p}_{1}$ or $\bar{p}_{2}$ with contiguity degree at least $\varepsilon$. We can assume that $t$ avoids $\Gamma^{\prime}$. Hence $\Pi$ and $\Gamma^{\prime}$ are subdiagrams of the (disc) diagram $\Gamma$. As in the proofs of Lemmas 9.3, 9.4, we can use Lemmas 8.3 and 9.1 and move $\Pi$ through either the trapezium $\Delta_{1}$ or the trapezium $\Delta_{2}$ leaving $\phi\left(p_{1}\right)$ and $\phi\left(p_{2}\right)$ the same modulo $Z(\mathbb{S}, r a)$, leaving paths $s_{i}, x_{i}$ untouched and decreasing the number of Burnside $r a$-cells in $\Gamma$. The proof now can be completed by induction on the number of Burnside ra-cells in $\Gamma$.

\subsection{Conditions (Z1), (Z2), (Z3)}

Now we are ready to check that the presentation $Z(\mathbb{S}, \Lambda, r a)$ satisfies conditions (Z1), (Z2), (Z3) from Section 3.

Here we are going to use notation from Section 3 again, as in Section 8.

Let $\mathcal{R}_{0}=\mathcal{S}_{0}$ be the set $Z(\mathbb{S}, r a)$ of all relations in $Z(\mathbb{S}, \Lambda, r a)$ except for the hub, and let $\mathcal{S}_{1 / 2}=\{\Lambda(0)\}$, so $Z(\mathbb{S}, \Lambda, r a)=\mathcal{R}_{1 / 2}$. We let $\mathcal{Y}=\mathbf{K}$, so all letters from $\mathbf{R} \cup \mathbf{A}$ are 0-letters.

Condition (Z1.1) holds by an easy inspection. Condition (Z1.2) holds by Proposition 8.2.

The length of $\Lambda$ is $(2 n+3) N>n$, so (Z2.1) holds. The condition (Z2.2) is obviously true.

The next lemma gives us (Z2.3).

Lemma 9.8. Assume that $v_{1} w_{1}$ and $v_{2} w_{2}$ are cyclic permutations of $\Lambda(0)^{ \pm 1}$ and $\left|v_{1}\right| \geq \varepsilon|\Lambda(0)|$. Then if $u_{1} v_{1}=v_{2} u_{2}$ holds modulo $\mathcal{S}_{0}$ for some 0 -words $u_{1}, u_{2}$, then we have $u_{2} w_{1}=w_{2} u_{1}$ modulo $\mathcal{S}_{0}$.

Proof. Suppose that $u_{1} v_{1}=v_{2} u_{2}$ modulo $\mathcal{S}_{0}$. Then there exists a van Kampen diagram $\Gamma$ over $\mathcal{S}_{0}$ with boundary $p_{1} q_{1} p_{2}^{-1} q_{2}^{-1}$ such that $\phi\left(p_{1}\right) \equiv u_{1}, \phi\left(p_{2}\right) \equiv u_{2}, \phi\left(q_{1}\right) \equiv v_{1}, \phi\left(q_{2}\right) \equiv v_{2}$ (see Figure 6). The $\mathbf{K}$-bands of $\Gamma$ starting on $q_{1}$ end on $q_{2}$ since $\phi\left(q_{1}\right)$ is a linear word and $u_{1}, u_{2}$ do not contain letters from $\mathbf{K} \cup \mathbf{K}^{-1}$. The labels of the start and end edges of each of these bands are equal. Hence $v_{2} \equiv \phi\left(q_{2}\right) \equiv \phi\left(q_{1}\right) \equiv v_{1} \equiv v$. Since $|v|>0$, and $\Lambda(0)$ and $\Lambda(0)^{-1}$ do not have common letters, $v$ cannot be a subword of both $\Lambda(0)$ and $\Lambda(0)^{-1}$. Without loss of generality assume that $v$ is a subword of $\Lambda(0)$.

Recall that for every $j=1, \ldots, N$, we denote $\Lambda_{j}(0)$ to be the subword of $\Lambda(0)$ starting with $\lambda(j)$ and ending with $\lambda(j+1)$ ( as usual " $N+1$ " here is 1 ).

By Lemma 9.6 there exists a word $h$ over $\mathbb{S}$ such that $v \cdot h=v, u_{1}=L\left(h, z_{1}\right), u_{2}=R\left(h, z_{2}\right)$ modulo $Z(\mathbb{S}, r a)$ for some $z_{1}, z_{2}$. Hence we can assume that $u_{1}=L\left(h, z_{1}\right), u_{2}=R\left(h, z_{2}\right)$ in the free group and that $\Gamma=T(v, h)$. Since $T(v, h)$ is a diagram over $Z(\mathbb{S}, a), \Gamma$ does not contain Burnside ra-cells. 
Since $v \equiv \phi\left(q_{1}\right) \equiv \phi\left(q_{2}\right)$ is a subword of $\Lambda(0)$, we can attach to $\Gamma$ two hubs $\Pi_{1}$ and $\Pi_{2}$ along the paths $q_{1}$ and $q_{2}$, and consider the resulting diagram $\Delta$ with boundary label $w_{2} u_{1} w_{1}^{-1} u_{2}^{-1}$. We need to show that $\Delta$ can be replaced by a diagram over $\mathcal{S}_{0}$ with the same boundary label.

Since $\left|v_{1}\right| \geq \varepsilon|\Lambda(0)|$ and $N>n>3 / \varepsilon$, we conclude that $v_{1}$ contains $\Lambda_{j}(0)$ for some $j>1$.

Let $q_{1}^{\prime}$ be the subpath of $q_{1}$ such that $\phi\left(q_{1}^{\prime}\right) \equiv \Lambda_{j}(0)$. Since $\Lambda$ is linear, every $\mathbf{K}$-band starting on $q_{1}$ ends on $q_{2}$. Let $\mathcal{T}_{1}$ be the $\lambda(j)$-band and $\mathcal{T}_{2}$ be the $\lambda(j+1)$-band starting on $q_{1}^{\prime}$. Then $\mathcal{T}_{1}$ ends on the edge of $q_{2}$ labeled by $\lambda(j, 0)$ and $\mathcal{T}_{2}$ ends on the edge of $q_{2}$ labeled by $\lambda(j+1,0)$.

The bands $\mathcal{T}_{1}, \mathcal{T}_{2}$, the path $q_{1}^{\prime}$ and the path $q_{2}$ bound a subdiagram $\Gamma^{\prime}$ of $\Gamma$ which is a trapezium. By Lemma 7.5, $\Gamma^{\prime}=T\left(\Lambda_{j}(0), h\right)$ and $\Lambda_{j}(0) \cdot h=\Lambda_{j}(0)$. Let $t$ be the subpath of $\partial\left(\Gamma^{\prime}\right)$ which is a side of $\mathcal{T}_{1}$.

Consider the subdiagram $\Delta^{\prime}$ of $\Delta$ consisting of $\Pi_{1}, \Pi_{2}$ and $t$. Since $\Delta \backslash \Delta^{\prime}$ consists of 0 -cells, it suffices to show that the boundary label of $\Delta^{\prime}$ is equal to 1 modulo $\mathcal{S}_{0}$.

Thus we need to show that $\phi(t)=L(h, \lambda(j))$ commutes with

$$
\Lambda_{j}(0) \lambda(j+1,0)^{-1} \Lambda_{j+1}(0) \lambda(j+2,0)^{-1} \ldots \Lambda_{j-1}(0) \lambda(j, 0)^{-1} .
$$

By Proposition 6.16, for every $i=1, \ldots, N$ we have $\Lambda_{i}(0) \cdot h=\Lambda_{i}(0)$. The trapezium $T\left(\Lambda_{i}(0), h\right)$ gives us the equality

$$
\Lambda_{i}(0)^{-1} L(h, \lambda(i)) \Lambda_{i}(0)=R(h, \lambda(i+1))=\lambda(i+1,0)^{-1}(L(h, \lambda(i+1)) \lambda(i+1,0), i=1, \ldots, N
$$

modulo $\mathcal{S}_{0}$. Hence

$$
\lambda(i+1,0) \Lambda_{i}(0)^{-1} L(h, \lambda(i)) \Lambda_{i}(0) \lambda(i+1,0)^{-1}=L(h, \lambda(i+1)), i=1, \ldots, N .
$$

Hence if we conjugate $L(h, \lambda(i))$ by the word (26), we get $L(h, \lambda(i))$ as required.

As in Section 3, for every cyclically reduced essential element $g \in H_{k r a}$ we define $\mathbf{0}(g)$ as the maximal subgroup of $H_{r a}$ which contains $g$ in its normalizer. For an arbitrary essential element $g$ of the form $v u v^{-1}$ where $u$ is cyclically reduced, we define $\mathbf{0}(g)=v \mathbf{0}(u) v^{-1}$. By Lemma 3.7, $\mathbf{0}(g)$ is well defined (does not depend on the presentation $g=v u v^{-1}$ ).

The following lemma will imply both (Z3.1) and (Z3.2).

Lemma 9.9. Let $w$ be an essential element represented by a cyclically minimal in rank $1 / 2$ word $W$, let $x \neq 1$ be a 0 -element in $H_{k r a}$ such that $w^{-4} x w^{4}$ is a 0 -element. Then the following two statements hold.

(i) There exists a 0 -element $u$ such that $w u^{-1}$ commutes with every element of $\mathbf{0}(w)$.

(ii) $x \in \mathbf{0}(w)$.

Proof. We divide the proof into several steps.

1. Let $P_{1}$ be a 0 -word representing $x$. By Lemma 3.4 every cyclic shift of $W$ is a cyclically $\mathbf{K}$-reduced word. Therefore we can assume that $W$ starts with a letter $z \in \mathbf{K} \cup \mathbf{K}^{-1}$ (by Lemma $3.7)$.

By Lemma 3.6, $(W z)^{-1} P_{1} W z$ is equal in $H_{k r a}$ to a 0 -word $P$. By Lemma $9.5 W z=C B z^{\prime} D$ in $H_{k r a}$ where $B$ does not contain $\mathbf{R}$-letters and starts with a $\mathbf{K}$-letter $z^{\prime}$ such that $C z^{\prime}=z C^{\prime}$, $z^{\prime} D=D^{\prime} z$ modulo $Z(\mathbb{S}, a)$ where $D^{\prime} \equiv L(f, z), C \equiv L\left(f^{\prime}, z\right)$ for some words $f, f^{\prime}$ over $\mathbb{S}$. 
In particular since $W z=C B z^{\prime} D=C B D^{\prime} z$, we have $W=C B D^{\prime}$ in $H_{k r a}$. Since $D^{\prime} C z^{\prime}=$ $z^{\prime} D C^{\prime}$, passing if necessary to a cyclic shift $B D^{\prime} C$ of $W$, we can assume that $C$ is empty, so $z^{\prime}=z$, and replace $W$ by $B D^{\prime}$ where $D^{\prime} z=z D$ modulo $Z(\mathbb{S}, a)$. The word $B$ is equal to $z B_{1} U$ where $B_{1}$ is either empty or ends with a letter $z^{\prime} \in \mathbf{K} \cup \mathbf{K}^{-1}, U$ is a word over $\mathbf{A}$.

2. By Lemma 3.6, we have the following equalities in $H_{k r a}$ :

$$
\begin{gathered}
\left(z B_{1} U D^{\prime} z B_{1} U z\right)^{-1} P_{1}\left(z B_{1} U D^{\prime} z B_{1} U z\right)=P_{2} \\
\left(z B_{1} U D^{\prime} z B_{1} U D^{\prime} z B_{1} U z\right)^{-1} P_{1}\left(z B_{1} U D^{\prime} z B_{1} U D^{\prime} z B_{1} U z\right)=P_{3}
\end{gathered}
$$

where $P_{2}$ and $P_{3}$ are 0 -words.

By Lemma 9.7 (which can be applied because $z B_{1} U D^{\prime} z B_{1} U z$ is reduced since this word is equal to a subword of the same length of the reduced word $W^{3}$ ) we can assume that equality (27) holds modulo $Z(\mathbb{S}, a)$ (replacing $P_{1}$ and $P_{2}$ by equal in $H_{k r a}$ words if necessary), $B z$ is a reduced admissible word. By Lemma 3.6, since $D^{\prime} z=z D$, we have that $\left(z B_{1} U z\right)^{-1} P_{1}\left(z B_{1} U z\right)$ is a 0 -word modulo $Z(\mathbb{S}, a)$. Hence by Lemma $9.6 P_{1}=L(h, z)$ modulo $Z(\mathbb{S}, a)$ and

$$
B z \cdot h=B z .
$$

3. Now consider a diagram $\Delta$ over $Z(\mathbb{S}, a)$ corresponding to equality $(27), \partial(\Delta)=p_{1} q_{1} p_{2}^{-1} q_{2}^{-1}$ where $\phi\left(p_{1}\right) \equiv P_{1}, \phi\left(p_{2}\right) \equiv P_{2}, \phi\left(q_{1}\right) \equiv B D^{\prime} B z \equiv \phi\left(q_{2}\right)$. Then $q_{1}=s_{1} t_{1} s_{1}^{\prime}$, where $\phi\left(s_{1}\right) \equiv B$, $\phi\left(t_{1}\right) \equiv D^{\prime}, \phi\left(s_{1}^{\prime}\right) \equiv B z$. Consider the maximal $z$-band $\mathcal{T}_{1}$ in $\Delta$ starting on the first edge of $s_{1}^{\prime}$. As usual, we can assume that $p_{2}$ is a side of a $z$-band $\mathcal{T}_{2}$. The bands $\mathcal{T}_{1}$ and $\mathcal{T}_{2}$ bound a subdiagram $\Delta_{1}$ of $\Delta$ which is a trapezium. By Lemma $7.5, \Delta_{1}=T\left(B z, h_{1}\right)$ where $h_{1}$ is the history of $\mathcal{T}_{1}$.

Consider the natural homomorphism $\psi$ from $H_{k r a}$ to the free group freely generated by $\mathbb{S}$. This homomorphism takes $\mathbf{K}$ and $\mathbf{A}$ to 1 , and takes each letter from $\mathbf{R}$ to the corresponding rule from $\mathbb{S}$. It is clear that this homomorphism is correctly defined because killing all letters from $\mathbf{K} \cup \mathbf{A}$ in every relation from $Z(\mathbb{S}, a)$ and replacing letters from $\mathbf{R}$ by the corresponding rules from $\mathbb{S}$ gives us a trivial relation $1=1$. Since $D^{\prime}=L(f, z), \psi\left(D^{\prime}\right)=f$. Applying this homomorphism to the boundary label of the subdiagram of $\Delta$ bounded by $p_{1}$ and a side of $\mathcal{T}_{1}$, we get $h_{1}=f^{-1} h f$. Hence we have

$$
B z \cdot f^{-1} h f=B z .
$$

4. Now let $\Delta_{1}$ be a van Kampen diagram corresponding to equality (28). Applying Lemma 9.6 to the subdiagram $\Delta_{2}$ of $\Delta_{1}$ bounded by two $z$-bands $\mathcal{T}_{1}, \mathcal{T}_{2}$ corresponding to the two last distinguished occurrences of $z$ in $z B_{1} U D^{\prime} z B_{1} U D^{\prime} z B_{1} U z$, we obtain $z B_{1} U z \cdot h_{2}=z B_{1} U z$, i.e. $B z \cdot h_{2}=B z$ where $h_{2}$ is the history of the $z$-band $\mathcal{T}_{1}$. Applying the homomorphism $\psi$ to the boundary label of the subdiagram of $\Delta_{2}^{\prime}=\Delta_{1} \backslash \Delta_{2}$ we obtain the equality $\psi\left(D^{\prime}\right)^{-2} h \psi\left(D^{\prime}\right)^{2} h_{2}^{-1}=$ $f^{-2} h f^{2} h_{2}^{-1}$ modulo Burnside relations. Hence $f^{-2} h f^{2} h_{2}^{-1}=1$ modulo Burnside relations. We conclude that

$$
B z \cdot h_{2}=B z
$$

where $h_{2}=f^{-2} h f^{2}$ modulo Burnside relations.

Let $W_{1}=B z, h_{1}=f^{-1} h f, g=f, b=h_{2}$. Then $W_{1} \cdot h_{1}=W_{1}$ by $(30), W_{1} \cdot g h_{1} g^{-1}=W_{1}$ by (29) and $W_{1} \cdot b=W_{1}$ by (31) where $b=g^{-1} h_{1} g$ modulo Burnside relations. 
Suppose that $B z$ contains an accepted subword $B^{\prime}$. Then $B^{\prime} \cdot h^{\prime}$ is a cyclic shift $C$ of $\Lambda(0)$ for some word $h^{\prime}$ over $\mathbb{S}$. Then for some admissible subword $B^{\prime \prime}$ of $B$ and $B^{\prime}, B^{\prime \prime} \cdot h^{\prime}=C^{\prime}$ where $C^{\prime} C^{\prime \prime} \equiv C,\left|C^{\prime \prime}\right| \leq 1$. By Lemma $7.5 B^{\prime \prime}=U C^{\prime} V$ in $H_{k r a}$ where $U$ and $V$ are 0 -words. Hence $B^{\prime \prime}=U\left(C^{\prime \prime}\right)^{-1} V$ in rank $1 / 2$. Since $\left|U\left(C^{\prime \prime}\right)^{-1} V\right|<\left|U C^{\prime} V\right|$, and $B^{\prime \prime}$ is a subword of $W$, we get a contradiction with the assumption that $W$ is cyclically minimal in rank $1 / 2$.

Thus all conditions of Proposition 6.17 hold. By that proposition and by Remark 6.18, we can conclude that for every integer $s$ there exists an word $b_{s+1}$ over $\mathbb{S}$ which is equal to $g^{s} h_{1} g^{-s}=$ $f^{-s-1} h f^{s+1}$ modulo Burnside relations and such that

$$
B z \cdot b_{s+1}=B z .
$$

5. For arbitrary integer $s$ consider the trapezia $T_{s}=T\left(B z, b_{s}\right)$. Let $\partial\left(T_{s}\right)=p_{1} q_{1} p_{2}^{-1} q_{2}^{-1}$, where $\phi\left(p_{1}\right)=L\left(b_{s}, z\right), \phi\left(p_{2}\right)=R\left(b_{s}, z\right), \phi\left(q_{i}\right)=B z, i=1,2$.

Considering the subdiagram of $T_{s}$ obtained by removing the last $z$-band (whose side is $p_{2}$ ), we get the equality

$$
L\left(b_{s}, z\right) B L\left(b_{s}, z\right)^{-1}=B
$$

modulo $Z(\mathbb{S}, a)$. Thus $B$ commutes with $L\left(b_{s}, z\right)$ modulo $Z(\mathbb{S}, a)$. Notice that $b_{0}=h$. Hence $B$ commutes with $L(f, z)^{-s} L(h, z) L(f, z)^{s}$ modulo $Z(\mathbb{S}, r a)$. Recall that $L(f, z) \equiv D^{\prime}, L(h, z) \equiv P_{1} \equiv$ $L\left(b_{0}, z\right)$. Hence $\left(D^{\prime}\right)^{-s} x\left(D^{\prime}\right)^{s}$ commutes with $B$ for every integer $s$.

Therefore for every non-negative integer $s$, we have the following equalities in $H_{k r a}$ :

$$
\begin{aligned}
& W^{-s} P_{1} W^{s}= \\
& \left(B D^{\prime}\right)^{-s} P_{1}\left(B D^{\prime}\right)^{s}=\left(D^{\prime}\right)^{-1} B^{-1} \ldots\left(D^{\prime}\right)^{-1} B^{-1}\left(D^{\prime}\right)^{-1}\left(B^{-1} P_{1} B\right) D^{\prime} B D^{\prime} \ldots B D^{\prime}= \\
& \left(D^{\prime}\right)^{-1} B^{-1} \ldots\left(D^{\prime}\right)^{-1} B^{-1}\left(\left(D^{\prime}\right)^{-1} P_{1} D^{\prime}\right) B D^{\prime} \ldots B D^{\prime}= \\
& \left(D^{\prime}\right)^{-1} B^{-1} \ldots B^{-1}\left(\left(\overline{\left.\left.D^{\prime}\right)^{-2} P_{1}\left(D^{\prime}\right)^{2}\right) B \ldots B} D^{\prime}=\ldots\right.\right. \\
& \left(D^{\prime}\right)^{-s} P_{1}\left(D^{\prime}\right)^{s}
\end{aligned}
$$

(we underline the parts of the words which are changing). Similar equalities hold for negative $s$. Therefore for every integer $s, w^{-s} x w^{s}$ is a 0 -element in $H_{k r a}$. Hence $x \in \mathbf{0}(w)$ (since the subgroup generated by $w^{-s} x w^{s}, s=0, \pm 1, \ldots$, consists of 0 -elements and is normalized by $\left.w\right)$.

6. Let us prove that $B$ commutes with every element $y \in \mathbf{0}(w)$ modulo $Z(\mathbb{S}, r a)$. Let $P_{y}$ be a word in $\mathbf{R} \cup \mathbf{A}$ representing $y$. Since $y \in \mathbf{0}(w), W^{-2} P_{y} W^{2}$ is equal modulo $Z(\mathbb{S}, r a)$ to a 0 -word. By Lemma 3.6, $\left(B D^{\prime} z\right)^{-1} P_{y}\left(B D^{\prime} z\right)$ is a 0 -word (modulo $Z(\mathbb{S}, r a)$ ). Since $D^{\prime} z=z D$ modulo $Z(\mathbb{S}, r a)$, we have that $(B z D)^{-1} P_{y}(B z D)$ is a 0 -word modulo $Z(\mathbb{S}, r a)$. By Lemma 3.6, $(B z)^{-1} P_{y}(B z)$ is a 0 -word $P_{y}^{\prime}$ modulo $Z(\mathbb{S}, r a)$. Considering a van Kampen diagram for this equality, we deduce (by a usual argument) that $P_{y}=L\left(h_{y}, z\right), P_{y}^{\prime}=R\left(h_{y}^{\prime}, z\right)$ for some words $h_{y}, h_{y}^{\prime}$ over $\mathbb{S}$. Applying the homomorphism $\psi$ to the equality

$$
(B z)^{-1} L\left(h_{y}, z\right)(B z)=R\left(h_{y}^{\prime}, z\right)
$$

we get that $h_{y}=h_{y}^{\prime}$ modulo Burnside relations. The last equality implies

$$
B^{-1} P_{y} B=B^{-1} L\left(h_{y}, z\right) B=z R\left(h_{y}^{\prime}, z\right) z^{-1}=L\left(h_{y}^{\prime}, z\right)=P_{y}
$$

modulo $Z(\mathbb{S}, r a)$ (by Lemma 7.1 ). Hence $B$ commutes with $P_{y}$ modulo $Z(\mathbb{S}, r a)$, as required. 
7. Now let $y \in \mathbf{0}(w)$ be represented by a word $P_{y}$. Let us prove by induction on $l$ that $\left(D^{\prime}\right)^{l} P_{y}\left(D^{\prime}\right)^{-l}$ belongs to $\mathbf{0}(w)$ for every $l \geq 0$. This is clearly true for $l=0$. Suppose that it is true for some $l \geq 0$. Then

$$
\left(D^{\prime}\right)^{l+1} P_{y}\left(D^{\prime}\right)^{-l-1}=\left(D^{\prime}\right)\left(D^{\prime}\right)^{l} P_{y}\left(D^{\prime}\right)^{-l}\left(D^{\prime}\right)^{-1}=B^{-1}\left(B D^{\prime}\right)\left(D^{\prime}\right)^{l} P_{y}\left(D^{\prime}\right)^{-l}\left(B D^{\prime}\right)^{-1} B .
$$

Since $\left(D^{\prime}\right)^{l} P_{y}\left(D^{\prime}\right)^{-l}$ belongs to $\mathbf{0}(w)=\mathbf{0}\left(B D^{\prime}\right)$, we have that $\left(B D^{\prime}\right)\left(D^{\prime}\right)^{l} P_{y}\left(D^{\prime}\right)^{-l}\left(B D^{\prime}\right)^{-1}$ belongs to $\mathbf{0}(w)$ (by the definition of $\mathbf{0}(w)$ ). Since $B$ commutes with every element of $\mathbf{0}(w)$, (34) implies that

$$
\left(D^{\prime}\right)^{l+1} P_{y}\left(D^{\prime}\right)^{-l-1}=\left(B D^{\prime}\right)\left(D^{\prime}\right)^{l} P_{y}\left(D^{\prime}\right)^{-l}\left(B D^{\prime}\right)^{-1} \in \mathbf{0}(w) .
$$

Similarly one can prove that $\left(D^{\prime}\right)^{l} P_{y}\left(D^{\prime}\right)^{-l}$ belongs to $\mathbf{0}(w)$ for all $l<0$.

8. Let $u$ be the element of $H_{k r a}$ represented by $D^{\prime}$. Then clearly $u$ is a 0 -element. Let $w$ be the element represented by $W\left(D^{\prime}\right)^{-1}$. We need to prove that $w$ commutes with $y$, that is, $W\left(D^{\prime}\right)^{-1}$ commutes with $P_{y}$ modulo $Z(\mathbb{S}, r a)$, or, equivalently,

$$
\left(B D^{\prime}\right)^{-1} P_{y}\left(B D^{\prime}\right)=\left(D^{\prime}\right)^{-1} P_{y}\left(D^{\prime}\right)
$$

modulo $Z(\mathbb{S}, r a)$. This has been proved already in (33).

This completes verification of properties (Z1), (Z2), (Z3). By Proposition 3.19, we have the following statement.

Proposition 9.10. There exists a graded presentation $\mathcal{R}_{k r a}$ containing $Z(\mathbb{S}, r a)$ of the factor group $H_{k r a}(\infty)$ of $H_{k r a}$ over the subgroup generated by all $n$-th powers of elements of $H_{k r a}$, such that every relation of $\mathcal{R}_{k r a}$ of rank $\geq 1$ has the form $u^{n}=1$ for some word $u$, and every minimal diagram over $\mathcal{R}_{\text {kra }}$ satisfies property A from Section 2.4, and all the lemmas from Section 3.

\section{Proof of Theorem 1.3}

Lemma 10.1. For every word $w\left(x_{1}, \ldots, x_{m}\right)$, if $w\left(a_{1}(\kappa(1)), \ldots, a_{m}(\kappa(1))\right)=1$ in $H_{k r a}(\infty)$, then $w\left(a_{1}, \ldots, a_{m}\right)=1$ in $\langle A(\kappa(1))\rangle$.

Proof. Let $\Delta$ be a g-reduced diagram over $\mathcal{R}_{k r a}$ with boundary label $w\left(a_{1}(\kappa(1)), \ldots, a_{m}(\kappa(1))\right)$. Suppose that $\Delta$ contains cells of rank $>0$. By Proposition $9.10 \Delta$ is an $A$-map. By Lemma 2.5 $\Delta$ contains a cell $\Pi$ of rank $>0$ and a contiguity subdiagram of $\Pi$ to $\partial(\Delta)$. Since non-0 letters in presentation $\mathcal{R}_{k r a}$ are letters from $\mathbf{K}$, by definition of contiguity subdiagrams from Section 2 , $\partial(\Delta)$ contains a $\mathbf{K}$-edge, a contradiction.

Hence $\Delta$ does not contain cells of rank $>0$. Therefore $\Delta$ is a diagram over $H_{k r a}$. So we can assume that $\Delta$ is a g-reduced diagram over $H_{k r a}$. By Lemma 9.2 we can assume that $\Delta$ does not contain $\mathbf{K}$-annuli. Hence $\Delta$ does not contain $\mathbf{K}$-edges. Therefore $\Delta$ is a diagram over $\mathcal{R}_{r a}$ where $\mathbf{R}$ is the set of non-0 letters.

Suppose that $\Delta$ contains a cell of rank $>0$. Then by Proposition 8.2 and Lemma $2.6 \Delta$ contains a cell $\Pi$ of rank $>0$ and a contiguity subdiagram of $\Pi$ to $\partial(\Delta)$. Hence $\partial(\Delta)$ contains $\mathbf{R}$-edges, a contradiction. Therefore $\Delta$ does not contain cells of rank $>0$. Hence $\Delta$ is a diagram over $H_{r a}$. By Lemma 7.3, part (3), $\Delta$ does not contain $\mathbf{R}$-annuli. Hence $\Delta$ does not contain $\mathbf{R}$-edges. This means that all cells of $\Delta$ are $a$-cells. Therefore $\Delta$ is a diagram over $H_{a}$. 
Since $\langle A(\kappa(1))\rangle$ is a retract of $H_{a}$, we obtain that $w\left(a_{1}(\kappa(1)), \ldots, a_{m}(\kappa(1))\right)$ is equal to 1 modulo $R(\kappa(1))$-relations and Burnside relations, as required.

Given the Burnside group $B(m, n)$ generated by $a_{1}, \ldots, a_{m}$, we have, by Lemma 5.2 , a homomorphism $\phi: B(m, n) \rightarrow \mathcal{H}$ such that $\phi\left(a_{i}\right)=a_{i}(\kappa(1)), i=1, \ldots, m$.

Assume that $\phi\left(w\left(a_{1}, \ldots, a_{m}\right)\right) \in \mathcal{H}^{n}$. Then $w\left(a_{1}(\kappa(1)), \ldots a_{m}(\kappa(1))\right)=1$ in $H_{k r a}(\infty)=$ $\mathcal{H}(n)=\mathcal{H} / \mathcal{H}^{n}$ by Proposition 9.10. Setting $R(\kappa(1))=\varnothing$, we have from Lemma 10.1 that $w=1$ in $\langle A(\kappa(1))\rangle=B\left(a_{1}, \ldots, a_{m} ; n\right)$. Hence $\phi$ is an embedding, and moreover, the intersection of $\phi(B(m, n)) \cap \mathcal{H}^{n}$ is trivial. This proves Statement (1) of Theorem 1.3.

Let now $L$ be an arbitrary normal subgroup of $B(m, n)$ (the latter can now be identified with its image in $\left.\mathcal{H} / \mathcal{H}^{n}\right)$. To prove Statement (2) of Theorem 1.3, we have to show that $L=L^{\mathcal{H} / \mathcal{H}^{n}} \cap$ $B(m, n)=L$. Let us define $R(\kappa(1))$ to be the set of all words in $a_{1}(\kappa(1)), \ldots, a_{m}(\kappa(1))$ representing elements of $L$. The new group $H_{k r a}(\infty)$ (whose construction depends now on the new choice of $R(\kappa(1)))$ is a homomorphic image of $\mathcal{H} / \mathcal{H}^{n}$ by Proposition 9.10 . Therefore it suffices to prove that every word $w\left(a_{1}(\kappa(1)), \ldots, a_{m}(\kappa(1))\right)$ that is equal to 1 in $H_{k r a}(\infty)$, is also trivial in $B(m, n) / L \cong$ $\langle A(\kappa(1))\rangle$. But again, this claim is a part of Lemma 10.1 .

The proof of Theorem 1.3 is complete.

\section{References}

[1] S.I. Adian. Random walks on free periodic groups. Izv. Akad. Nauk SSSR, Ser. Mat. 46 (1982), no. $6,1139-1149$.

[2] S.I. Adian. The Burnside problem and identities in groups. Ergebnisse der Mathematik und ihrer Grenzgebiete [Results in Mathematics and Related Areas], 95. Springer-Verlag, BerlinNew York, 1979, 311 pp.

[3] S.I. Adian. Periodic products of groups. Number theory, mathematical analysis and their applications. Trudy Mat. Inst. Steklov. 142 (1976), 3-21, 268.

[4] S. Banach, A. Tarski. Sur la décomposition de ensembles de points an parties respectivement congruentes. Fund. math 6 (1924), 244-277.

[5] J. C. Birget, A.Yu. Ol'shanskii, E.Rips, M. V. Sapir. Isoperimetric functions of groups and computational complexity of the word problem", 1998 (accepted in Annals of Mathematics), preprint is available at http://www.math.vanderbilt.edu/ msapir/publications.html.

[6] M. G. Brin and C. C. Squier. Groups of piecewise linear homeomorphisms of the real line. Invent. Math., 79 (1985), no. 3, 485-498.

[7] J. W. Cannon, W. J. Floyd and W. R. Parry. Introductory notes on Richard Thompson's groups. L'Enseignement Mathématique (2) 42 (1996), no. 3-4, 215-256.

[8] A.H. Clifford and G.B. Preston, The algebraic theory of semigroups. Vol. I. Mathematical Surveys, No. 7 American Mathematical Society, Providence, R.I. 1961

[9] J. M. Cohen. Cogrowth and amenability of discrete groups. J. Funct. Anal. 48 (1982), no. 3, 301-309 
[10] Mahlon M. Day. Amenable semigroups. Illinois J. Math. 1 (1957), 509-544.

[11] E. Følner. On groups with full Banach mean value. Math. Scand. 3 (1955), 243-254.

[12] Open problems in infinite-dimensional topology. Edited by Ross Geoghegan. The Proceedings of the 1979 Topology Conference (Ohio Univ., Athens, Ohio, 1979). Topology Proc. 4 (1979), no. 1, 287-338 (1980).

[13] F. P. Greenleaf. Invariant means on topological groups and their applications. Van Nostrand Reinhold, New York, 1969.

[14] R.I. Grigorchuk. Symmetrical random walks on discrete groups. Multicomponent random systems. Adv. Probab. Related Topics, 6, Dekker, New York, 1980, 285-325.

[15] R. I. Grigorchuk. An example of a finitely presented amenable group that does not belong to the class EG. Mat. Sb., 189(1) (1998) 79-100.

[16] F. Hausdorff. Grunzüge der Mengenlehre. Leipzig, 1914.

[17] S. V. Ivanov. On HNN-extensions in the class of groups of a large odd exponent. Preprint, 2002 .

[18] S. V. Ivanov and A.Y. Ol'shanskii. Hyperbolic groups and their quotients of bounded exponents. Trans. Amer. Math. Soc. 348 (1996), no. 6, 2091-2138.

[19] Harry Kesten. Full Banach mean values on countable groups. Math. Scand. 7 (1959), 146-156.

[20] Harry Kesten. Symmetric random walks on groups. Trans. Amer. Math. Soc. 92 (1959), 336354.

[21] O.G. Kharlampovich and M.V. Sapir. Algorithmic problems in varieties. Internat. J. Algebra Comput. 5 (1995), no. 4-5, 379-602.

[22] Kourovka Notebook. Unsolved Problems in Group Theory. 8th edition, Novosibirsk, 1982.

[23] Roger Lyndon and Paul Schupp. Combinatorial group theory. Springer-Verlag, 1977.

[24] K.V.Mikhajlovskii. Some generalizations of the HNN-construction in the periodic case, \#1063B94, VINITI, Moscow, 1994, 59 pp.

[25] J. von Neumann. Zur allgemeinen Theorie des Masses. Fund. math., 13 (1929), 73-116.

[26] P. S. Novikov and S.I. Adian. Infinite periodic groups. I,II, III, (Russian) Izv. Akad. Nauk SSSR Ser. Mat. 321968 212-244, 251-524, 709-731.

[27] A. Yu. Ol'shanskii. An infinite simple torsion-free Noetherian group. Izv. Akad. Nauk SSSR Ser. Mat. 43 (1979), no. 6, 1328-1393.

[28] A.Yu. Ol'shanskii. An infinite group with subgroups of prime order. Izvestia Akad. Nauk SSSR, Ser. Mat., 44(1980), no. 2, 309-321. 
[29] A. Yu. Ol'shanskii. On the question of the existence of an invariant mean on a group. (Russian) Uspekhi Mat. Nauk 35 (1980), no. 4(214), 199-200.

[30] A. Yu. Ol'shanskii. The geometry of defining relations in groups, Nauka, Moscow, 1989.

[31] A. Yu. Ol'shanskii. The SQ-universality of hyperbolic groups, Mat. Sb., 1995, v. 186, N 8, 119-132.

[32] A. Yu. Ol'shanskii. On distortion of subgroups in finitely presented groups. Mat. Sb., 1997, V.188, N 11, 51-98.

[33] A. Yu. Ol'shanskii, M. V. Sapir. Embeddings of relatively free groups into finitely presented groups. Contemp. Math., 264, 2000, 23-47.

[34] A.Yu. Ol'shanskii and M.V. Sapir. Length and Area Functions on Groups and Quasi-Isometric Higman Embeddings. To appear, IJAC, 2000.

[35] M. V. Sapir. Problems of Burnside type and the finite basis property in varieties of semigroups. Izv. Akad. Nauk. SSSR. Ser. Mat., 51(2), 1987, 319-340.

[36] M. V. Sapir, J. C. Birget, E. Rips. Isoperimetric and isodiametric functions of groups, 1997, accepted in Annals of Mathematics, preprint available at http://www.math.vanderbilt.edu/ msapir/publications.html.

[37] W. Specht. Zur Theorie der messbaren Gruppen. Math. Z., 74 (1960), 325-366.

[38] J. Tits. Free subgroups of linear groups. J. Algebra (20) 1972, 250-270.

[39] A.M. Vershik. Comments to papers by J. von Neumann, in "J. von Neumann, Selected works in functional analysis", v1, Moscow, Nauka, 1987, 357-376. 


\section{Subject index}

accepted admissible word 70

adjacent edges 13

admissible word 55, 62, 65

annulus 12

$(S, T)$-annulus 12

$c$-aperiodic word 42

K-band 83

$\overline{\mathbf{K}}$-band 83

R-band 83

$S$-band 11

$\tau$-band 83

bases of a trapezium 85

bond 13

0-bond 13

bottom side of a band 11

Burnside cells 47

Burnside-reduced word 65

Burnside ra-relations 90

0-cell 10

chamber 93

cleaning rule 57

compatibility of cells 28,47

congruence extension property (CEP) 4

connecting line of a band 11

connecting line of a bond 13

connecting line of a contiguity submap 15

connecting rule 54,57

contiguity arc 15

contiguity degree 15

contiguity submap 14

converting $S$-rules into sets of relations 56

$\Omega$-coordinate of a word 55

copy of a word 63

crossing bands 12

cyclically $Y$-reduced element 20

cyclically $Y$-reduced word 21

diagram divided by a connecting line (band) 12

domain of a word in $\mathbb{S} 63$

0 -edge 10

0-element 20

essential element 20

graded map 10

graded presentation 20

history of a computation 8 history of a $\mathbf{K}$-band 83

history of a trapezium 85

hoop 47

homomorphism $\vartheta_{i} 71$

homomorphism $v 71$

inside diagram of an annulus 12

inverse rule 56

inverse semigroup $P(\mathbb{S}) 62$

Lowest Parameter Principle 4

length of a path 7

$Y$-length 19

$Y$-letter 19

0-letter 11

map 10

A-map 15

narrow bond 17

normal trapezium 85

$j$-pair 28

1/2-pair 28

period of rank $i 27,46$

$A$-periodic word 27

phase decomposition 27

positive edge (cell) 10

positive and negative $S$-rules 56

principal cell of a bond 13

properties A1, A2, A3 15

properties (M1),(M2), (M3) 10

properties (T1), (T2), (T3) 93

properties (TR1), (TR2) 85

properties (Z1),(Z2), (Z3) 20

rank of a cell 10

rank of a map 10

reduced admissible word 62

reduced band 12

reduced path 12

g-reduced diagram 28

$Z(\mathbb{S}, a)$-reduced diagram 84

*-reduced word 52

$Y$-reduced word 21

$T$-relator 46

$a$-relations 82

$k$-relations 82

$r$-relations 82

ra-relations 83

$S$-rule 55 
$S$-rule applicable to an admissible word 55 $S$-rule locking a sector 55

$S$-rule left (right) active for a sector 55 section of a contour of a diagram 13 $\varepsilon$-section 45

\section{$H$-section 47}

sector 55

sector of the type (form) $\left[z z^{\prime}\right] 68$

simple in rank $i$ word 28, 46

smooth section 15

$\alpha$-series 18

side arc of a contiguity subdiagram 15

sides of a trapezium 85

start and end edges of a band 11

0-subgroup 20

top side of a band 11

trapezium 85

$\psi$-trivial word 52

type of a map (diagram) 10

van Kampen diagram 11

van Kampen lemma 11

vertices in the same phase 30

0 -word 20

$H$-word 45

working rule 54,57
$H_{k r a}(\infty) 101$

$\overline{\mathbf{K}} 54$

$\overline{\mathbf{K}}(z) 54$

K 54

$\Lambda 54$

$\Lambda_{i}(0) 67$

$L(\tau, z) 55$

$L^{a}(\tau, z) 63$

$\mathbf{0}(g) 21$

$\Omega 54$

$\left(\Pi_{1}, \Gamma, \Pi_{2}\right) 15$

$\Pi \circ \Theta 91$

$R(\kappa(1)) 62$

$R(\tau, z) 55$

$R^{a}(\tau, z) 63$

R 54

$\mathbf{R}(z) 56$

$\mathcal{R}(\infty) 28$

$\mathcal{R}_{i} 27$

$\mathcal{R}_{\text {kra }} 101$

$\mathcal{R}_{\text {ra }} 88$

$r$ (П) 10

$r(q) 15$

$r(\tau, z) 57$

$\mathcal{S}_{i} 27$

$\mathbb{S}^{+} 54,57$

$\mathbb{S}_{\omega}^{+} 54$

$\sim_{i} 27$

$\Theta * \Pi 90$

$\stackrel{\ell}{\rightarrow} 55$

$\overline{\tau(z)} 54$

$\operatorname{top}(\mathcal{B}) 11$

$U(\omega) 54$

$W \cdot h 63$

X 54

$\mathcal{X}_{i} 27$

$z_{-}, z_{+} 55$

$Z(\mathbb{S}) 61$

$Z(\mathbb{S}, \Lambda) 61$

$Z(\mathbb{S}, a) 83$

$Z(\mathbb{S}, r a) 90$

$Z(\mathbb{S}, \Lambda, r a) 90$

$H^{*} 62$

$H_{\text {ra }} 86$

$H_{r a}(\infty) 88$

$H_{k r a} 90$ 


\author{
Alexander Yu. Ol'shanskii \\ Department of Mathematics \\ Vanderbilt University \\ olsh@math.vanderbilt.edu \\ http://www.math.vanderbilt.edu/ olsh \\ and \\ Department of Higher Algebra, MEHMAT \\ Moscow State University \\ olshan@shabol.math.msu.su
}

\author{
Mark V. Sapir \\ Department of Mathematics \\ Vanderbilt University \\ http://www.math.vanderbilt.edu/ msapir
}

